Florida International University FIU Digital Commons

FIU Electronic Theses and Dissertations

University Graduate School

$11-17-2017$

\title{
Antibaryon Photoproduction using CLAS at Jefferson Lab
}

William B. Phelps

Florida International University, willphelps@gmail.com

DOI: 10.25148 /etd.FIDC004063

Follow this and additional works at: https://digitalcommons.fiu.edu/etd

Part of the Nuclear Commons

\section{Recommended Citation}

Phelps, William B., "Antibaryon Photoproduction using CLAS at Jefferson Lab" (2017). FIU Electronic Theses and Dissertations. 3507. https://digitalcommons.fiu.edu/etd/3507

This work is brought to you for free and open access by the University Graduate School at FIU Digital Commons. It has been accepted for inclusion in FIU Electronic Theses and Dissertations by an authorized administrator of FIU Digital Commons. For more information, please contact dcc@fiu.edu. 


\title{
FLORIDA INTERNATIONAL UNIVERSITY \\ Miami, Florida
}

\section{ANTIBARYON PHOTOPRODUCTION USING CLAS AT JEFFERSON LAB}

A dissertation submitted in partial fulfillment of the requirements for the degree of DOCTOR OF PHILOSOPHY

\author{
in \\ PHYSICS \\ by \\ William Phelps
}


To: Dean Michael R. Heithaus

College of Arts, Sciences, and Education

This dissertation, written by William Phelps, and entitled Antibaryon Photoproduction using CLAS at Jefferson Lab, having been approved in respect to style and intellectual content, is referred to you for judgment.

We have read this dissertation and recommend that it be approved.

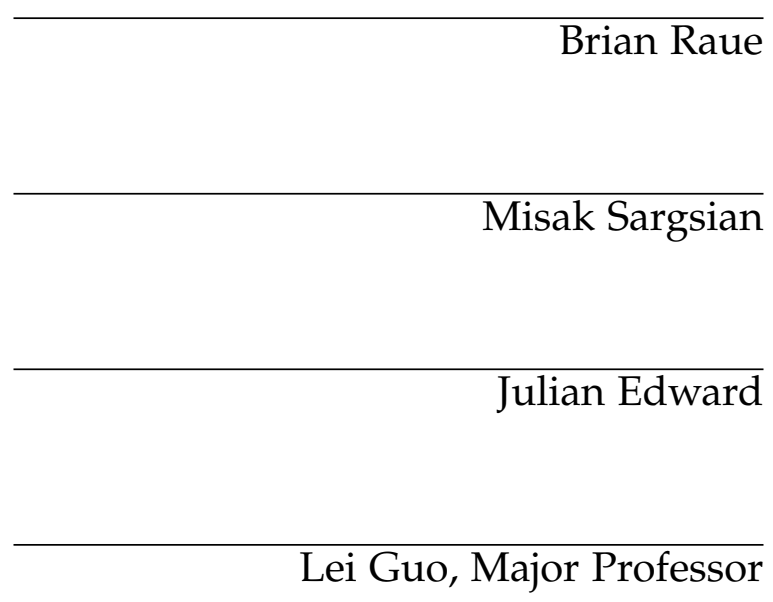

Date of Defense: November 17, 2017

The dissertation of William Phelps is approved.

Dean Michael R. Heithaus

College of Arts, Sciences, and Education

Andrés G. Gil

Vice President for Research and Economic Development and University Graduate School

Florida International University, 2017 
(C) Copyright 2017 by William Phelps

All rights reserved. 


\section{DEDICATION}

To my parents. 


\section{ACKNOWLEDGMENTS}

First of all, I would like to thank my advisor Lei Guo for all of the advice and patience he has given me over the years. Lei was always ready to help when he could and provided the much needed support (not to mention some serious life coaching skills). I will forever be grateful and indebted to him for everything that he has taught me.

I would also like to thank Carlos Salgado and Youri Sharabian for being excellent mentors at Jefferson Lab. The enormous amount of time and effort they dedicated to my education at the lab is priceless.

The $g_{12}$ collaborators and Jefferson Lab staff who devoted countless hours to make this experiment a success were absolutely crucial, thank you. Many thanks to those post docs and grad students who made the experiment possible.

At FIU I was surrounded with the most supportive group of colleagues and friends. I would like to give a special thanks to the nuclear group down at FIU including Adam, Rafael, Marianna, Jason, Eric, Hari, Dipak, and Puneet. The conversations and sharing of knowledge was crucial to my development as a physicist and I would not have made it without them.

A special thanks is in order for those who read my dissertation to provide feedback, Adam Freese, Rafael Badui, Florian Hauenstein, Daniel Lersch, and Especially Lei Guo and Brian Raue. I would also like to thank my committee members for all of their comments and feedback.

Daniel, Florian, Maxime, Stuart, Juan, Carlos, and Jenn, thank you all for your friendship and for putting up with my antics towards the end of writing, it would have been a much rougher ride without you all. 
And I could not thank my extremely supportive family enough for helping me all along the way. I would not have made it this far without you guys. It's over now, we can get back to a more normal life. 


\author{
ABSTRACT OF THE DISSERTATION \\ ANTIBARYON PHOTOPRODUCTION USING CLAS AT JEFFERSON LAB \\ by \\ William Phelps \\ Florida International University, 2017 \\ Miami, Florida \\ Professor Lei Guo, Major Professor
}

Antibaryon production has been investigated since the advent of nuclear physics, largely motivated by the potential for baryon-antibaryon bound states. Due to the recent availability of high statistics experiments there has been a renewed interest in how antibaryons are created in photoproduction. The $g_{12}$ (E04-005) experiment conducted at Thomas Jefferson National Accelerator Facility used the CEBAF Large Acceptance Spectrometer to provide the world's largest dataset for baryon-antibaryon photoproduction on a liquid hydrogen target. The focus of this work is to investigate the photoproduction mechanism of $\gamma p \rightarrow p p \bar{p}$ through detailed differential cross section measurements from 3.95 to $5.45 \mathrm{GeV}$. These first time results as well as the resulting total cross section measurement are reported. In particular the energy dependence $\left(\sigma\left(E_{\gamma}\right)\right)$ and the angular dependence $\left(d \sigma / d \cos \left(\theta_{\bar{p}}^{C M}\right)\right)$ have interesting features that have never been seen before. On the other hand, the mass distributions $(d \sigma / d M(p p)$ and $d \sigma / d M(p \bar{p})$ do not show evidence for previously reported narrow resonances. In addition, the first time total cross section mesurement of an antineutron in photoproduction using the reaction $\gamma p \rightarrow p p \bar{n} \pi^{-}$is reported. The significance and implications of these results will be discussed as well. 


\section{TABLE OF CONTENTS}

CHAPTER

PAGE

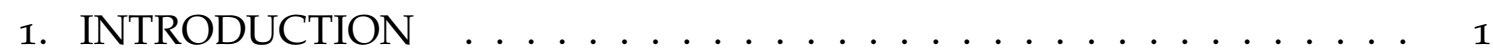

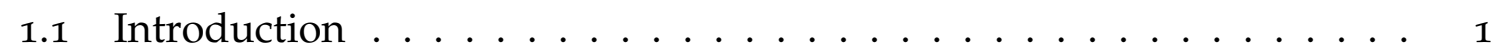

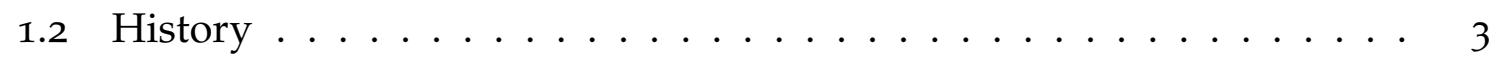

1.2.1 Recent Work . . . . . . . . . . . . . . . . . . . 7

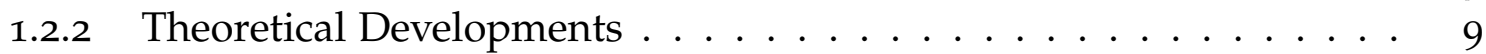

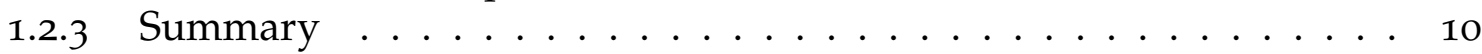

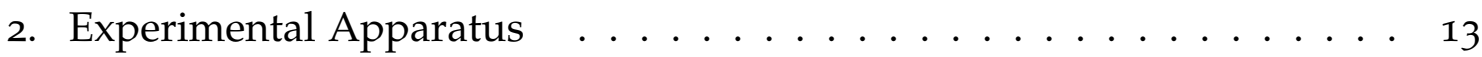

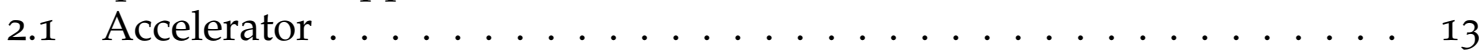

2.1.1 Overview of the Accelerator . . . . . . . . . . . . . . 13

2.1.2 Superconducting Radio-Frequency Cavities . . . . . . . . . . . . . 15

2.2 Tagger and Beamline . . . . . . . . . . . . . . . 16

2.3 CLAS Detector. . . . . . . . . . . . . . . . . . . 18

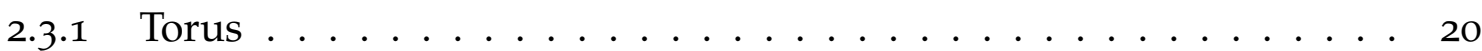

2.3 .2 Start Counter . . . . . . . . . . . . . . . . 20

2.3 .3 Drift Chambers . . . . . . . . . . . . . . . . . 23

2.3 .4 Time-of-Flight Counters . . . . . . . . . . . . . . . . . . . . . . . . . . . . . . . . .

2.3 .5 Cherenkov Counter. . . . . . . . . . . . . . . . . 25

2.3 .6 Electromagnetic Calorimeter . . . . . . . . . . . . . 26

2.4 Data Acquisition . . . . . . . . . . . . . . . . . . . . . . . . . . . . . . . . . . . . . . . . . .

2.5 The $g 12$ experiment . . . . . . . . . . . . . . . . . . . . . . . . . . . . . . . . . . . . . . .

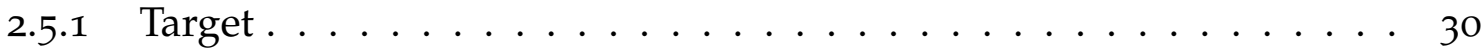

2.6 Event Reconstruction . . . . . . . . . . . . . . . . . 31

3. Data Analysis . . . . . . . . . . . . . . . . . . . . . . . . . . . . . . . . . . . .

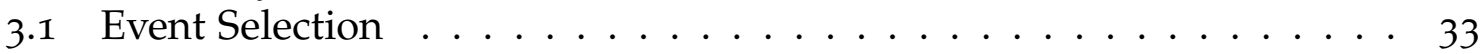

3.1 .1 Data Selection Cuts . . . . . . . . . . . . . . . . . 33

3.1 .2 Target Fiducial Cut . . . . . . . . . . . . . . . . . . . . . . . . . . . . . . . . 34

3.1.3 Detector Fiducial Cut . . . . . . . . . . . . . . . . . . . . . . . . . . . . . . . .

3.1.4 Time-of-Flight Knock Outs . . . . . . . . . . . . . . . . . . . . . . . . . . . . . . . . . 36

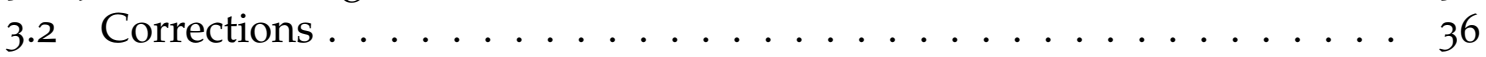

3.2 .1 Energy Loss . . . . . . . . . . . . . . . . . . . . . . . . . . . . . . . . . . . .

3.2.2 Momentum Correction . . . . . . . . . . . . . . . . . . . . . . . . . . . . . . .

3.2.3 Beam Energy Correction . . . . . . . . . . . . . . . . . . 39

$3 \cdot 3$ Reaction Reconstruction and Signal Extraction . . . . . . . . . . . . 40

3.3.1 Missing mass technique ......................... 40

3.4 Kinematic Fitting . . . . . . . . . . . . . . . . . . 4 4

3.4.1 Kinematic fitting results . . . . . . . . . . . . . . . . . 41

3.4.2 Event Selection with Kinematic Fitting . . . . . . . . . . . . . . 42

3.5 General Features of the data . . . . . . . . . . . . . . 43 
3.5 .1 Mass distributions $\ldots \ldots \ldots \ldots \ldots \ldots$

3.6 Monte Carlo Simulation . . . . . . . . . . . . . . . . . . . . 44

4. Cross section measurement of $\gamma p \rightarrow p p \bar{p} \ldots \ldots \ldots \ldots \ldots$

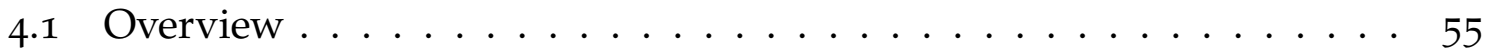

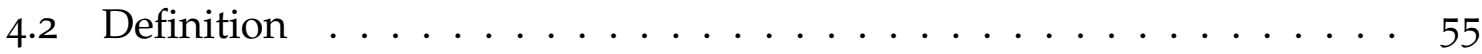

4.2 .1 Yield Extraction . . . . . . . . . . . . . . . . . 56

4.3 Simulation and Acceptance Calculation . . . . . . . . . . 56

4.3 .1 Weighting Program . . . . . . . . . . . . . . . . . . 57

4.3 .2 Quality Control . . . . . . . . . . . . . . . . . . . . . . 58

4.3 .3 Technical Considerations . . . . . . . . . . . . . . . . . 60

4.3 .4 Track Efficiency Correction . . . . . . . . . . . . . . . . . . . . 68

4.4 Differential Cross Sections . . . . . . . . . . . . . . . . . . . . . 68

$4.4 .1 \quad$ Mass Distributions of Events . . . . . . . . . . . . . . . . . 68

4.4 .2 Angular Distributions of Events $\ldots \ldots \ldots \ldots \ldots$

4.5 Total Cross Section Measurement. . . . . . . . . . . . . . . . . . 74

4.6 Cross Sections with $\gamma p \rightarrow p \bar{p}(p) \ldots \ldots \ldots \ldots \ldots$

4.6 .1 Comparison with Theory $\ldots \ldots \ldots \ldots \ldots$

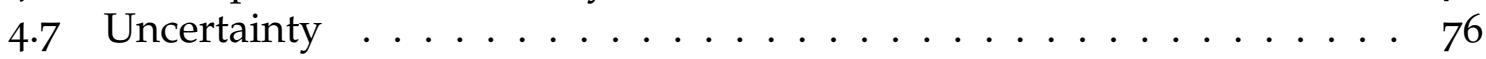

$4 \cdot 7 \cdot 1$ Statistical Uncertainty $\ldots \ldots \ldots \ldots \ldots \ldots$. . . . . . . . . . . 76

4.7 .2 Cut-based Systematic Uncertainty $\ldots \ldots \ldots \ldots \ldots$

$4.7 \cdot 3$ Model-Dependence of the Simulation $\ldots \ldots \ldots \ldots \ldots$

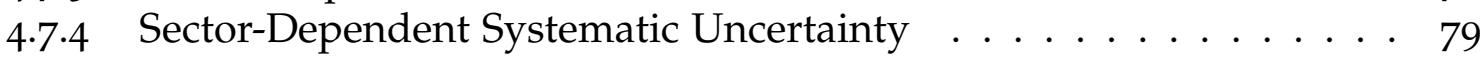

$4.7 \cdot 5$ Photon Flux Systematic Uncertainty $\ldots \ldots \ldots$. . . . . . . . . . . 79

4.7 .6 Track Efficiency Systematic Uncertainty . . . . . . . . . . . . . . . . 82

$4 \cdot 7 \cdot 7$ Systematic Uncertainty Summary $\ldots \ldots$. . . . . . . . . . . . 82

5. Cross section measurement of $\gamma p \rightarrow p p \bar{n} \pi^{-} \ldots \ldots \ldots$. . . . . 84

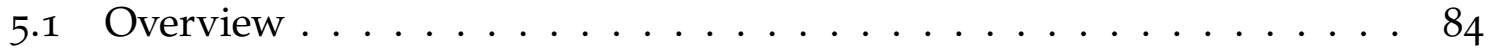

5.2 Simulation and Acceptance Calculation $\ldots \ldots \ldots \ldots \ldots$. . . . . 85

5.3 Total Cross Section Measurement . . . . . . . . . . . . . . . . . . 85

6. Discussion and Conclusions $\ldots \ldots \ldots \ldots \ldots$

A. Appendices . . . . . . . . . . . . . . . . . . . . . . . 95

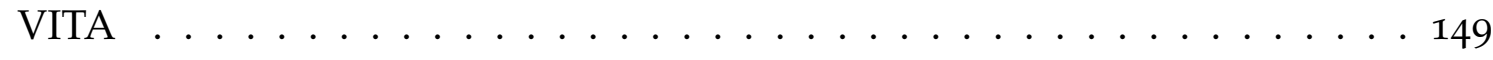




\section{LIST OF TABLES}

TABLE

PAGE

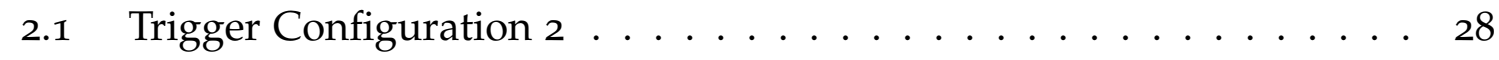

2.2 The running conditions of the $g 12$ experiment from [23] $\ldots \ldots \ldots$

3.1 List of cuts applied and the affect on the signal/(signal+background) ratio for the reaction $\gamma p \rightarrow p p(\bar{p}) \cdot \ldots \ldots \ldots \ldots \ldots$

3.2 Yield of events for the two topologies used after all cuts were applied. 34

3.3 Table of Gaussian widths and means from a fit to the kinematic fit parameter pull distributions from Figures 3.10 and 3.11 . The experimental data shows a shift in the mean which could be due to the momentum corrections not being suited for this reaction. $\cdots 4^{2}$

4.1 Constants used for calculating the cross sections. . . . . . . . . 56

$4.2 \quad$ List of systematic uncertainties. $\ldots \ldots \ldots \ldots \ldots$

$4 \cdot 3$ List of global systematic uncertainties. . . . . . . . . . . . 83 


\section{LIST OF FIGURES}

FIGURE

PAGE

1.1 The Standard Model of particle physics showing the three generations of quarks and leptons, and the Gauge and Scalar Bosons.

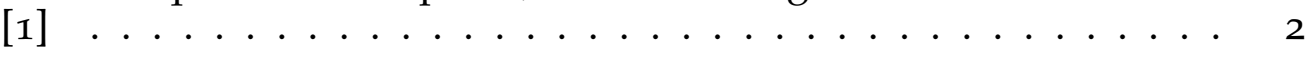

1.2 The invariant mass distributions for $p \bar{p}$ in the reaction $\pi^{-} p \rightarrow p_{f} \pi^{-}[p \bar{p}]$ using a 9 and $12 \mathrm{GeV} \pi^{-}$beam. a) Shows all events, b) shows events with an mass selection, selecting the $\Delta(1232)$ region in the $p_{\text {fast }} \pi^{-}$invariant mass, c) shows events with an mass selection, selecting the $N(1520)$ region in the $p_{\text {fast }} \pi^{-}$invariant mass, and d) showing the mass distribution without the $\Delta(1232)$ and $N(1520)$ mass selections in the $p_{\text {fast }} \pi^{-}$invariant mass. [6] . . . . . . . 5

1.3 Published world data for total cross sections for the reaction $\gamma p \rightarrow{ }^{\prime}$

1.4 The invariant mass distributions for $p \bar{p}$ in the photoproduction reaction $\gamma p \rightarrow p p \bar{p}[8]$. This distribution suggests a possible 2.02 $\mathrm{GeV}^{2}$ resonance in photoproduction. $\ldots \ldots \ldots \ldots$

1.5 The invariant mass distributions for $p \bar{p}$ using a combination of all 3 and 4 prong datasets that were studied in the reanalysis of WA56 data in pion production $[9] . \ldots \ldots \ldots . \ldots . \ldots$

1.6 Evidence for narrow resonances from an unpublished analysis note in the CLAS collaboration, from the G6b run. $[13] \ldots \ldots . . .99$

1.7 The invariant mass distribution of $p_{\text {fast }} \bar{p}$ showing statistically significant structure from a previous CLAS experiment, g6c. [11] $\ldots . .10$

1.8 Resonances seen in the $p \bar{p}$ mass spectrum from BESIII in the reaction $\pi / \Psi \rightarrow p \bar{p} \gamma .[6] \ldots \ldots \ldots \ldots \ldots \ldots \ldots \ldots \ldots$

1.9 Feynman diagrams for $p \bar{p}$ photoproduction used for the calculation of the predictions in $[14] \ldots \ldots \ldots \ldots \ldots$ II

1.10 Predictions for differential cross section $d \sigma / d t$ from several different models at GlueX energies $\left(E_{\gamma}=9 \mathrm{GeV}\right)[14] . \ldots \ldots \ldots 12$

2.1 Jefferson Lab site aerial photo from May 2016 showing the CEBAF Accelerator, Engineering Buildings and office buildings. This photo shows the original three experimental halls at the lower left and the newest addition, Hall-D, at the lower right. The data for this work were taken in Hall-B, at the center of the original

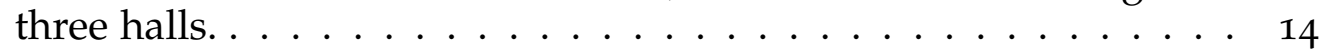


2.2 The CEBAF Accelerator overview. Showing the construction of the tunnels, injector, two linacs with the cryomodules, and the recirculating arcs with bending magnets. $\ldots \ldots \ldots \ldots$ 15

2.3 A pair of niobium cavities. . . . . . . . . . . . 15

\begin{tabular}{|rr|r|}
\hline 2.4 & A SRF cavity showing the charge on the inner surfaces from the \\
\hline standing wave. This potential difference accelerates the electrons. & 16
\end{tabular}

2.5 CLAS photon tagger. After electrons interact with a radiator and create bremsstrahlung photons they are bent into the scintillating paddles giving precise information about the timing and energy of the recoil electrons with energies $20-95 \%$ of the incident electron beam energy. . . . . . . . . . . . . . . 18

2.6 Three-dimensional model of Hall-B showing one of the six sectors in an exploded view with the CLAS detector shown in the center of the hall. The beam, shown in red, enters the hall from the lower right and passes through to the upper left. . . . . . . . . . 19

2.7 The CEBAF Large Acceptance Spectrometer (CLAS). The orientation of the figure has the beam entering from the upper left and exits to the beam dump on the lower right. . . . . . . . . . . . . . 19

2.8 The torus field lines looking downstream at the midplane of CLAS. It is important to note that the major component of the field lines is in the azimuthal direction. . . ...........21

2.9 The CLAS torus field map, parallel to the beamline, at half of the max production current. Also shown is the target placed at -90 cm from CLAS center. . . . . . . . . . . . . . 22

2.10 The upgraded, highly-segmented CLAS Start counter. Inside the start counter the $40 \mathrm{~cm}$ long target cell is visible.[17] $\ldots \ldots \ldots 22$

\begin{tabular}{|c|c|c|}
\hline 2.11 A CLAS Event Display view of a track going through two superlayers \\
\hline of the drift chambers. $\ldots \ldots \ldots \ldots$
\end{tabular}

2.12 The 57 Time of Flight (TOF) scintillating paddles for one sector [19]. . 25

2.13 Cross-sectional view of a Cherenkov Counter sector. The track that is shown is an electron collection trajectory [20]. . . . . . . 26

\begin{tabular}{|r|r|}
\hline 2.14 & One sector of the electromagnetic calorimeter exploded to show the \\
\hline internal construction and arrangement of the layers [22]. . . . . . 27
\end{tabular} 
2.15 A diagram showing the trigger logic for one sector of CLAS. The ST $\times$ TOF can only determine if there was a single track in the trigger, so the multitrack trigger requirement requires the tracks to be in different sectors. The EC $\times$ CC trigger was used for Lepton

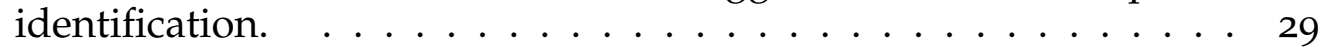

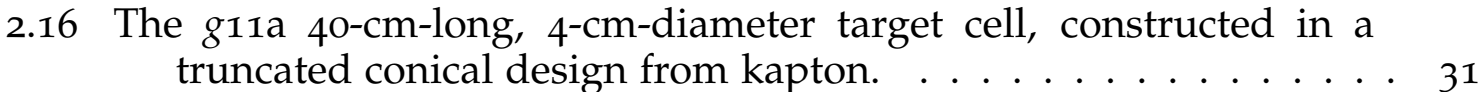

3.1 Missing mass off of $p p$ showing a missing $\bar{p}$, for the reaction $\gamma p \rightarrow$ \begin{tabular}{l|l}
$p p(\bar{p})$ which includes events where the $\bar{p}$ was also reconstructed. & 35
\end{tabular}

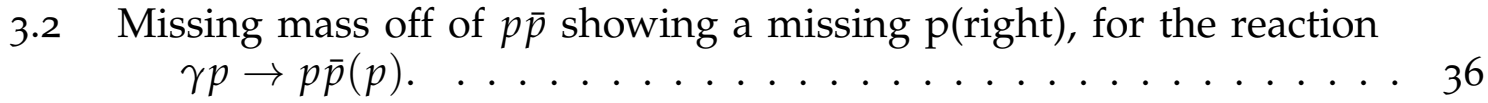

3.3 The event $z$ vertex distribution after all corrections and antiproton selection. Cuts were placed at the physical limits of the target from $-110 \mathrm{~cm}$ to $-70 \mathrm{~cm}$. $\ldots \ldots \ldots \ldots \ldots$

3.4 The event $x$ vertex vs $y$ vertex distribution after all corrections and antiproton selection. Cuts were placed at the physical limits of the target which has a radius of $2 \mathrm{~cm} . \ldots \ldots \ldots 38$

3.5 The angular distribution of the two proton tracks showing the holes in the detector; this is after applying fiducial cuts with the final

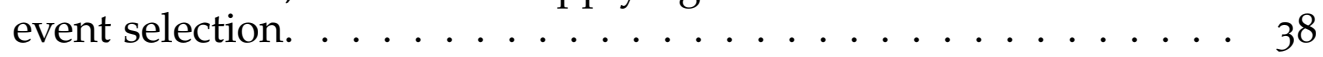

3.6 The missing mass as a function of the $\cos (\theta)$ of the fast proton showing an angular dependence of the momentum reconstructed by the drift chambers resulting in a skewness to the missing mass

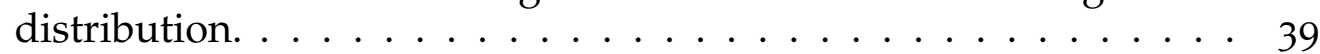

3.7 The missing mass as a function of the $\cos (\theta)$ of the fast proton after momentum corrections, showing a reduced skewness. However, due to the high momentum of the particles studied, the standard momentum corrections are insufficient and it is seen that there is little difference before and after applying the momentum correction. $\ldots \ldots \ldots \ldots \ldots$

3.8 The missing proton mass distribution by run number in the exclusive reaction $\gamma p \rightarrow p \pi^{+} \pi^{-}$showing the effects of the energy loss package [23]. The missing proton mass is lower after the correc$\begin{array}{ll}\text { tion due to the pions having higher momenta after the correction. } & 47\end{array}$

3.9 The mass dependence of the missing antiproton by run, before beam energy corrections shown in black and after beam energy corrections in blue. . . . . . . . . . . . . . . . 48 
3.10 Kinematic fitting pull distributions and confidence level showing sigmas near 1 but with shifts in the mean of the distribution. Shown with a $1 \%$ cut on the pull probability distribution to reduce background. See Table 3.3 for Gaussian fit parameters. $\ldots . . . .48$

3.11 Kinematic fitting pull distributions and confidence level distribution for Monte Carlo events. With means near zero and Gaussian sigmas near 1.0 it shows that the fitter is tuned well for this reaction. Shown with a $1 \%$ cut on the pull probability distribution to re\begin{tabular}{ll|l|l|} 
duce background. See Table & $3 \cdot 3$ & for Gaussian fit parameters. & . . . 49
\end{tabular}

3.12 Missing mass off of $p p$ showing a missing $\bar{p}$, for the reaction $\gamma p \rightarrow$ $p p(\bar{p})$. The red histogram shows the background distribution where the pull probability cut $<5 \%$ and the blue distribution shows the signal with a pull probability cut of $>5 \% . \ldots . . .50$

3.13 Beam energy distribution of events with all corrections and cuts applied. At $4.4 \mathrm{GeV}$ the trigger configuration changes and explains the sharp jump in the number events above $4.4 \mathrm{GeV}$. Additionally, the dips above $4.4 \mathrm{GeV}$ are due to inefficient paddles and are not seen in the total cross section. $\ldots \ldots \ldots . \ldots 5^{1}$

3.14 This figure shows the particle momentum distributions in comparison with one another. Detected particle momenta are all between $500 \mathrm{MeV}$ and $3 \mathrm{GeV} \ldots \ldots \ldots . \ldots \ldots 2$

3.15 Momentum vs $\beta$ distribution showing no out of time bands that are $\begin{array}{ll}\text { typically evidence of misidentified protons after all analysis cuts. } & 52\end{array}$

3.16 The invariant mass of the two protons for the final event selection. . . 53

$\begin{array}{r}3.17 \text { The invariant mass of the slow proton-antiproton pair for the final } \\ \hline \text { event selection. } \ldots \ldots \ldots \ldots \ldots\end{array}$

$\begin{array}{r}3.18 \text { The invariant mass of the fast proton-antiproton pair for the final } \\ \hline \text { event selection. } \ldots \ldots \ldots \ldots\end{array}$

3.19 The invariant mass of all combinations of the proton-antiproton pairs for the final event selection. ............ 54

4.1 Mass of the two protons vs $\cos \left(\theta_{\bar{p}}^{*}\right)$ cross section measurement which is used as one part of the model for the generator. $\ldots . . . .62$

4.2 Mass of the two protons vs $\cos \left(\theta_{\bar{p}}^{*}\right)$ prior to weighting, showing how the prior distribution must be taken into account before weighting with a new distribution. $\ldots \ldots \ldots \ldots 3$ 
4.3 Mass of the two protons vs $\cos \left(\theta_{\bar{p}}^{*}\right)$ after weighting which now match the cross section. ..................... 64

\begin{tabular}{|l}
\hline 4.4 Photon beam energy distribution comparison between Monte Carlo \\
\hline events and experimental data. $\ldots \ldots \ldots \ldots$
\end{tabular}

4.5 Angular distribution of the antiproton comparison between Monte Carlo events and experimental data. .......... 65

4.6 Angular distribution of the slow proton comparison between Monte Carlo events and experimental data. . . . . . . . . . 66

4.7 Angular distribution of the fast proton comparison between Monte Carlo events and experimental data. . . . . . . . . . . 66

4.8 Distribution of t-meson comparison between Monte Carlo events and experimental data. . . . . . . . . . . . . . 67

4.9 Distribution of t-baryon comparison between Monte Carlo events and experimental data. . . . . . . . . . . . . . . . 67

4.10 Differential cross section for $\gamma p \rightarrow p p \bar{p}$ showing the mass distributions as a function of beam energy. Histograms showing the phase space mass distributions are also shown in the same color as the graphs. Only statistical uncertainties are shown. . . . . . . . 69

4.11 Feynman diagrams showing $p \bar{p}$ production models with a meson exchange model (t-channel) on the left and a baryon exchange

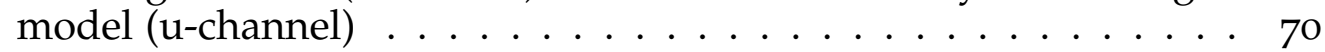

4.12 First measurement of the differential cross section for $\gamma p \rightarrow p p \bar{p}$ showing the angular distribution of the antiproton in the center of momentum frame as a function of beam energy. The fits are to Legendre polynomials $\ldots \ldots \ldots \ldots$ 71

4.13 First measurement of the differential cross section for $\gamma p \rightarrow p p \bar{p}$ showing the angular distribution of the antiproton in the center of momentum frame as a function of beam energy. The fits are to Legendre polynomials. $\ldots \ldots \ldots \ldots 7^{2}$

4.14 Legendre polynomial coefficients for $d \sigma / d \cos \left(\theta_{\bar{p}}\right) . \quad \ldots \ldots$. . . . 73

4.15 Legendre polynomial coefficient ratios from the fit to $d \sigma / d \cos \left(\theta_{\bar{p}}\right)$. The ratio shows how the shape of the differential cross section varies with beam energy $d \sigma / d \cos \left(\theta_{\bar{p}}\right)$. Overall the angular distribution becomes more uniform as the photon energy increases. $\quad 73$ 
4.16 Total cross section for $\gamma p \rightarrow p p \bar{p}$ comparing the measurement that is this work to existing world data from DESY and LAMP2 [27] [8].] 75

4.17 Total cross section for $\gamma p \rightarrow p p \bar{p}$, calculated using the missing proton data, comparing the measurement that is this work to existing world data from DESY and LAMP2 [27] [8]. Only statistical uncertainties are shown. ................... 75

4.18 Total cross section for $\gamma p \rightarrow p p \bar{p}$, calculated using the missing proton data, comparing the measurement that is this work to existing

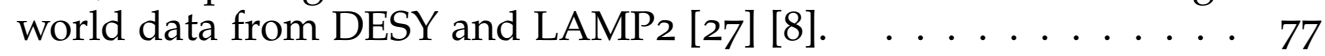

4.19 The run-by-run stability of the photon flux for all production runs excluding single track trigger runs. $\ldots . . . . . . . .80$

4.20 Comparison of the flux normalized yield for all beam currents, showing no beam current dependent systematic uncertainty with a slope consistent with $0.1 . \ldots . \ldots . \ldots$. . . . . . . .

4.21 The flux normalized yield for the two highest statistics beam currents. The $\sigma$ is correlated with the statistical uncertainty and the difference in means is used as the systematic uncertainty. . . . . . 81

$5.1 \quad$ Production model used for simulation. . . . . . . . . . . . . 84

5.2 Comparison of MC and data. . . . . . . . . . . . 85

$5 \cdot 3$ Total cross section for the reaction $\gamma p \rightarrow p p \bar{n} \pi^{-} . \ldots . \ldots 6$ 


\section{CHAPTER 1}

\section{INTRODUCTION}

\subsection{Introduction}

The Standard Model describes elementary particles and how they interact through the electromagnetic, strong, and weak forces. Each force is mediated by particles: the electromagnetic force by photons, the weak force by $W$ and $Z$ bosons, and the strong force by gluons (Fig. 1.1). Governed by these forces are three generations of quarks and leptons. Each generation of quarks contains two quarks and each generation of leptons contains a lepton and neutrino. The first generation of quarks starts with the up and down quarks that make up most visible matter as well as three generations of leptons that start with electrons. These particles all have antiparticles. If the particles are charged the antiparticle has an opposite charge. If the particle has a neutral charge then the antiparticle will also have a neutral charge.

Quantum Chromodynamics (QCD) describes how particles interact via the strong force [2]. The theory describes the color charge quantum number, in which each particle has a color, which is not to be confused with natural color. Within QCD only colorless states are allowed. The colors are red, green, and blue for matter and anti-red, anti-green, and anti-blue for antiparticles. A hadron is formed from colorless combinations of quarks and gluons as a result of a 


\section{Standard Model of Elementary Particles}

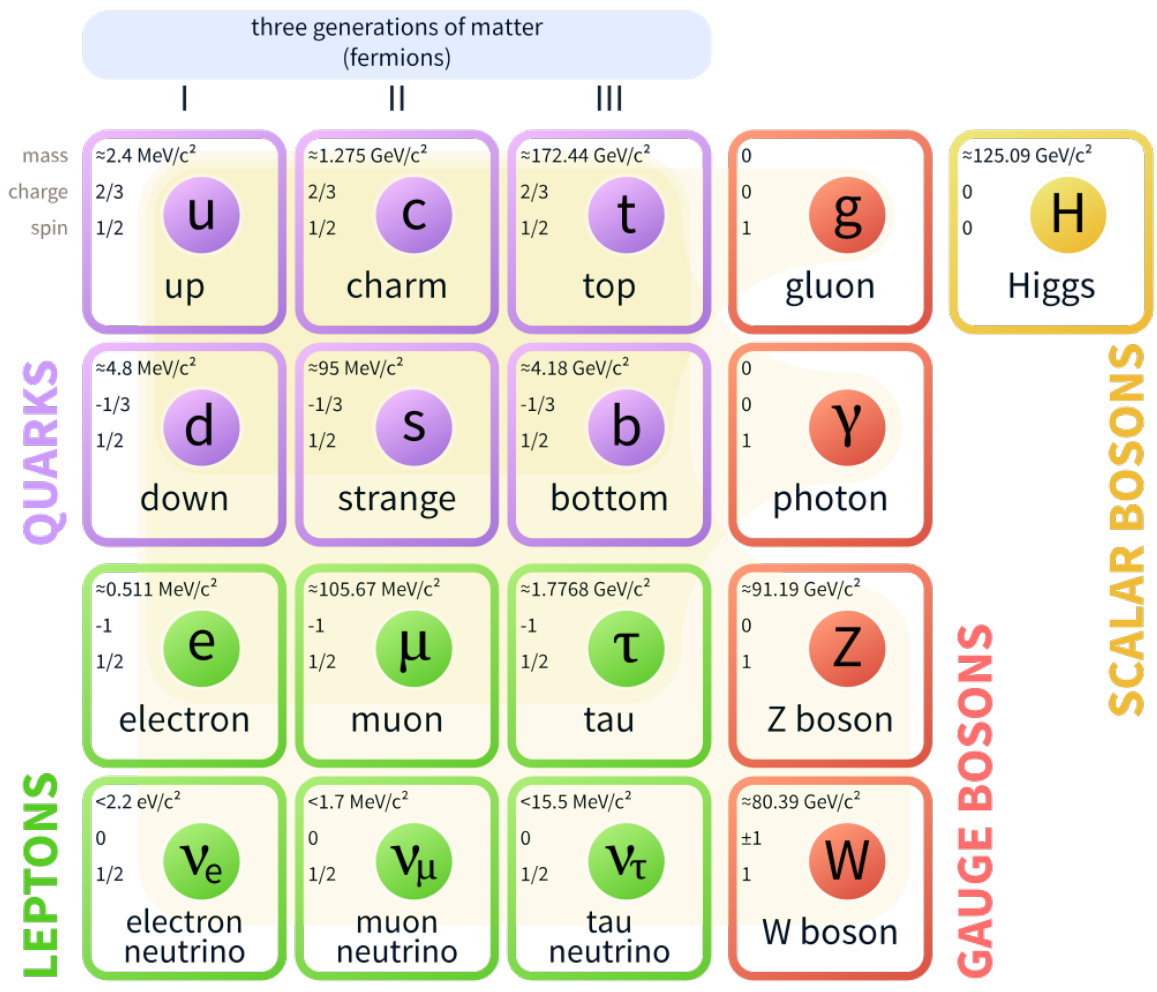

Figure 1.1: The Standard Model of particle physics showing the three generations of quarks and leptons, and the Gauge and Scalar Bosons. [1]

phenomenon known as color confinement. Color confinement states that color charged particles cannot be isolated since if additional energy were added to the system it would spontaneously pull one or more quark-antiquark pairs out of the vacuum in order to preserve a color neutral hadron. Color confinement is one of the least understood phenomena in nuclear physics today and facilities are being developed specifically to address this. Within QCD, two common hadrons are formed with three quarks called a baryon and mesons are formed from a quark and anti-quark. An example of a baryon is found in a hydrogen nucleus, a proton, and it is formed from two up quarks and one down quark $(u u d)$ where the colors must be red, blue, and green to form a colorless state. 
Much like the atomic spectrum which is used to study Quantum Electrodynamics, hadron spectroscopy is used to study the spectrum of hadronic states and it plays an important role in the understanding of Quantum Chromodynamics. Specifically, baryon-antibaryon production has been used to search for unusual resonances since the early days of nuclear physics. The history of these searches will be presented in the following section.

The main focus of this work is to explore the reaction $\gamma p \rightarrow p p \bar{p}$ using a high statistics photoproduction experiment at Thomas Jefferson National Lab (Jefferson Lab) using the Cebaf Large Acceptance Spectrometer (CLAS). Jefferson Lab is a national lab funded through the Department of Energy Office of Science. The lab, accelerator, and detector specifications and details will be discussed in Chapter 2 following the history of the reaction.

\subsection{History}

In 1932 Carl Anderson observed particles bending the opposite direction to electrons in a cloud chamber, which was the first observation of antimatter in the form of a positron or anti-electron [3]. Not long after the discovery of the positron, it was hypothesized that other particles also may have antimatter counterparts, and in Paul Dirac's Nobel Prize lecture he predicted the existence of antiprotons [4]. It took 22 years for Emilio Segre and Owen Chamberlain to experimentally confirm the existence of antiprotons in 1954 using the Bevatron particle accelerator at UC Berkeley [5]. Since then various reactions reactions that have produced antiprotons have been studied but the production mechanism is still not well understood. An intermediate state, "baryonium", has been speculated to exist where baryons and antibaryons are in a bound state and is reported as a narrow resonance [6, 7, 9]. 
These narrow resonances decaying to $p \bar{p}$ have been a heavily disputed topic and there have been more null results than confirmations in recent years. In the late 1970 's, using a negatively charged pion beam at CERN's Omega spectrometer, there were two reported resonances at 2.02 and $2.2 \mathrm{GeV}$ seen in Fig. 1.2 [6]. The reaction where the resonances were observed is $\pi^{-} p \rightarrow p_{f} \pi^{-}[p \bar{p}]$ using a 9 and $12 \mathrm{GeV} \pi^{-}$beam. Additional evidence for these resonances was observed when there was a $\Delta(1232)$ or $N(1520)$ present in the invariant mass distribution of the $p_{f} \pi^{-}$as seen in Fig. 1.4. However, there is a lack of evidence in the invariant mass spectrum without a $N^{*}$ or $\Delta$. The evidence for narrow states was later seen by a followup experiment two years later [7].

During the same time period there were several photoproduction experiments. In 1980 the LAMP2 spectrometer at Daresbury was a higher statistics experiment with approximately 200 events for the reaction $\gamma p \rightarrow p p \bar{p}$. The LAMP2 experiment showed invariant mass distributions with enhancements at 2.02 GeV as well as measured a total cross section for the reaction $\gamma p \rightarrow p p \bar{p}$ from threshold to $4.8 \mathrm{GeV}$ which is shown in comparison to later experiments in Fig. 1.3. The second higher statistics photoproduction experiment took place at DESY using a $7 \mathrm{GeV}$ electron synchrotron, which was equipped with a photon tagger. The reaction $\gamma p \rightarrow p p \bar{p}$ was studied using this tagged photon beam with photon energies from 4.7 to $6.6 \mathrm{GeV}[8]$. The experiment observed around 200 events and claimed to see the $2.02 \mathrm{GeV}$ resonance. In addition to evidence for narrow resonances, this photoproduction experiment measured the total cross section for the reaction $\gamma p \rightarrow p p \bar{p}$ from 4.7 to $6.6 \mathrm{GeV}$ and is the last published photoproduction experiment that investigated $\gamma p \rightarrow p p \bar{p}$.

To date no experiments have published differential cross sections due to a 


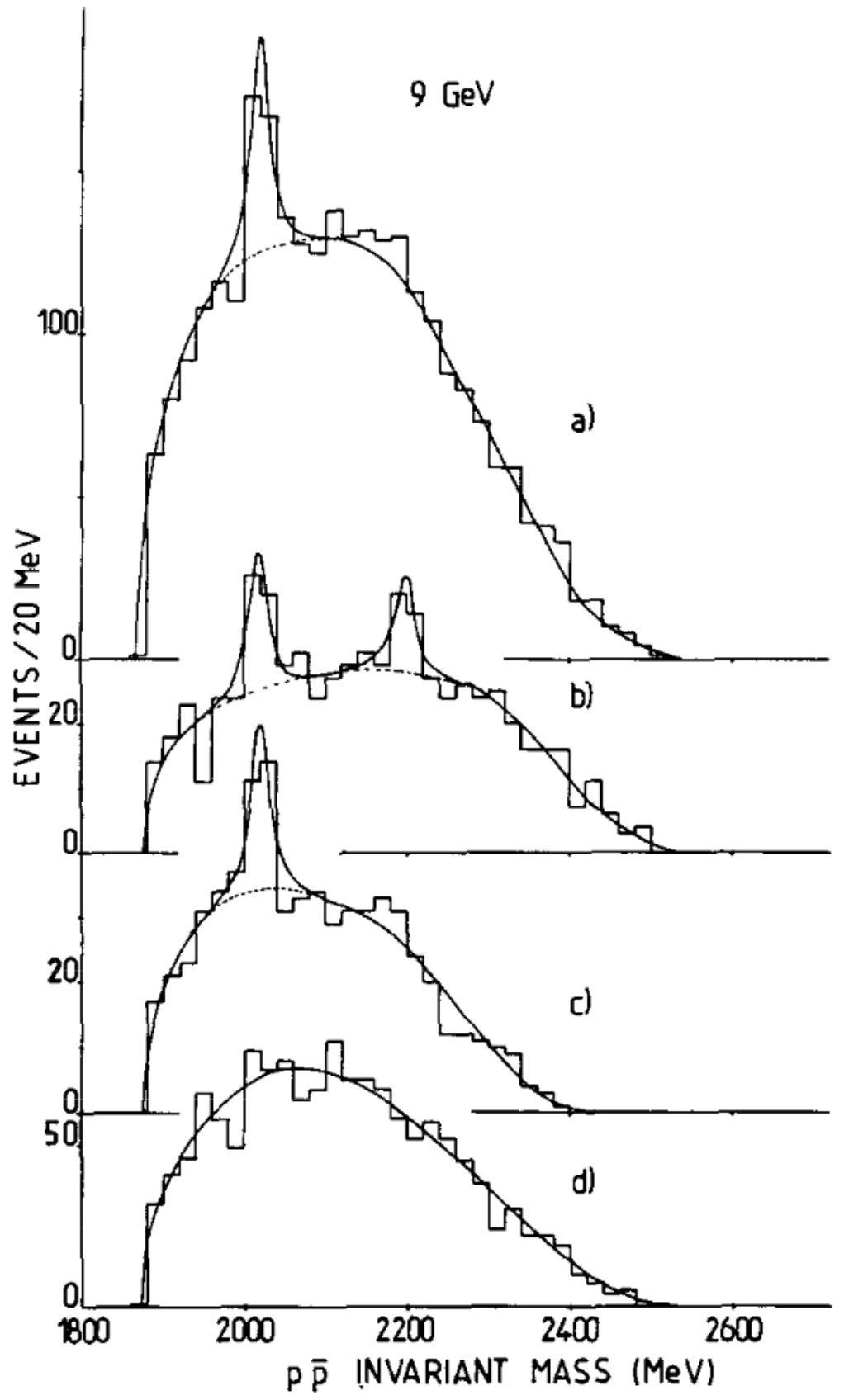

Figure 1.2: The invariant mass distributions for $p \bar{p}$ in the reaction $\pi^{-} p \rightarrow$ $p_{f} \pi^{-}[p \bar{p}]$ using a 9 and $12 \mathrm{GeV} \pi^{-}$beam. a) Shows all events, b) shows events with an mass selection, selecting the $\Delta(1232)$ region in the $p_{\text {fast }} \pi^{-}$invariant mass, c) shows events with an mass selection, selecting the $N(1520)$ region in the $p_{\text {fast }} \pi^{-}$invariant mass, and d) showing the mass distribution without the $\Delta(1232)$ and $N(1520)$ mass selections in the $p_{\text {fast }} \pi^{-}$invariant mass. [6] 

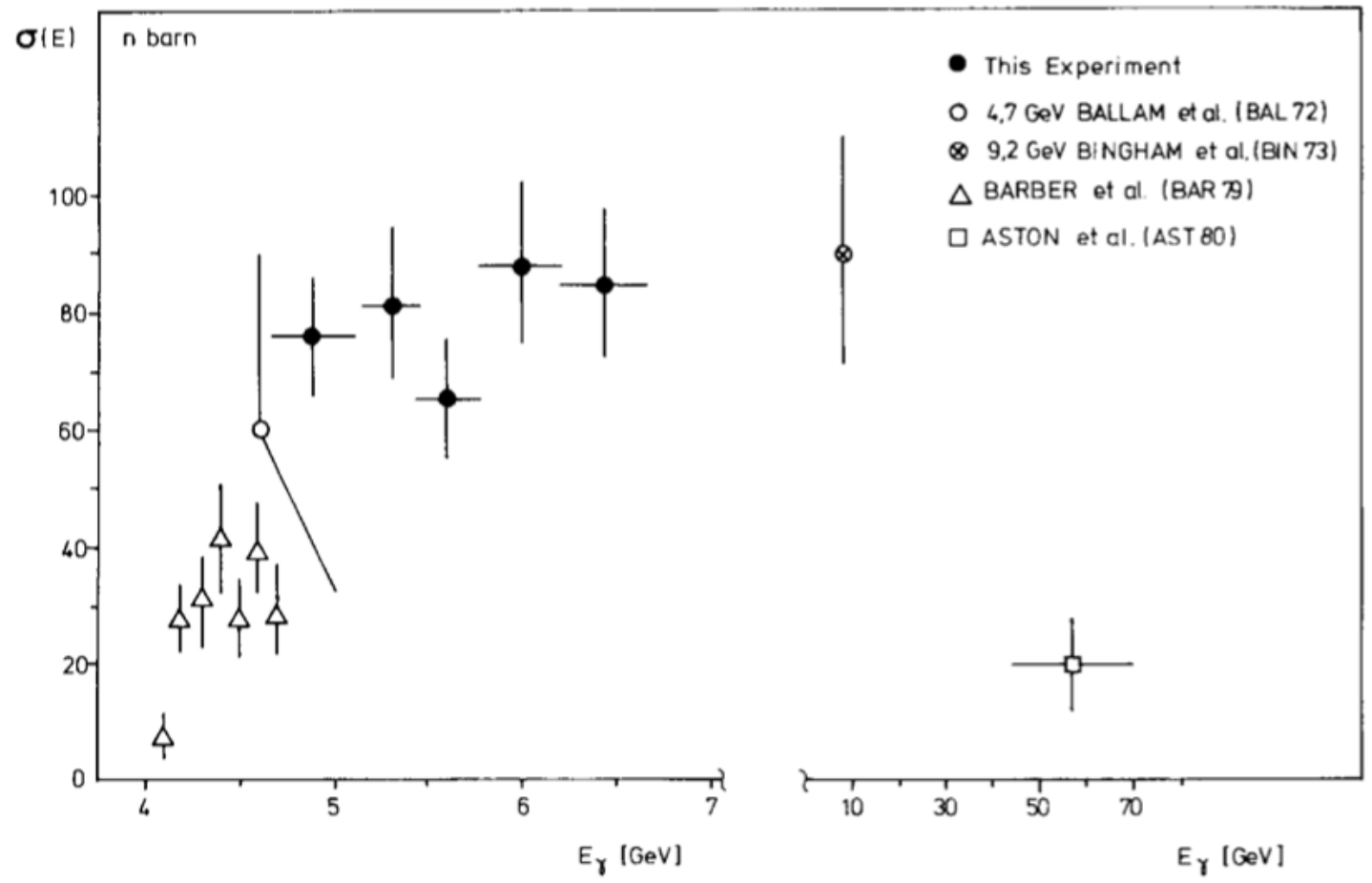

Figure 1.3: Published world data for total cross sections for the reaction $\gamma p \rightarrow$ $p p \bar{p}[8]$.

lack of both statistics and a realistic simulation model. In order to understand the production mechanism of $p \bar{p}$ differential cross sections are clearly needed.

In 1999 a reanalysis of the WA56 experiment data from the $\Omega$ spectrometer at CERN published a paper that claimed to have comprehensive evidence for a narrow $p \bar{p}$ state at $2.02 \mathrm{GeV}$ as seen in Figure 1.5 [9]. The experiment was conducted using a 12 and $20 \mathrm{GeV} \pi^{+}$beam. The primary reaction studied was $\pi^{+} p \rightarrow p_{\text {fast }} \pi^{+} p \bar{p}$ with several other reactions with either an additional $\pi^{0}$ or an additional $\pi^{+} \pi^{-}$pair. Cross sections for these reactions were not reported.

In addition to narrow resonances, broad resonances have been reported in proton-antiproton $(p \bar{p})$ annihilation experiments such as the Low Energy Antiproton Ring (LEAR) at CERN [10]. These broad resonances have not yet been confirmed or reported in photoproduction experiments [11]. In annihilation ex- 


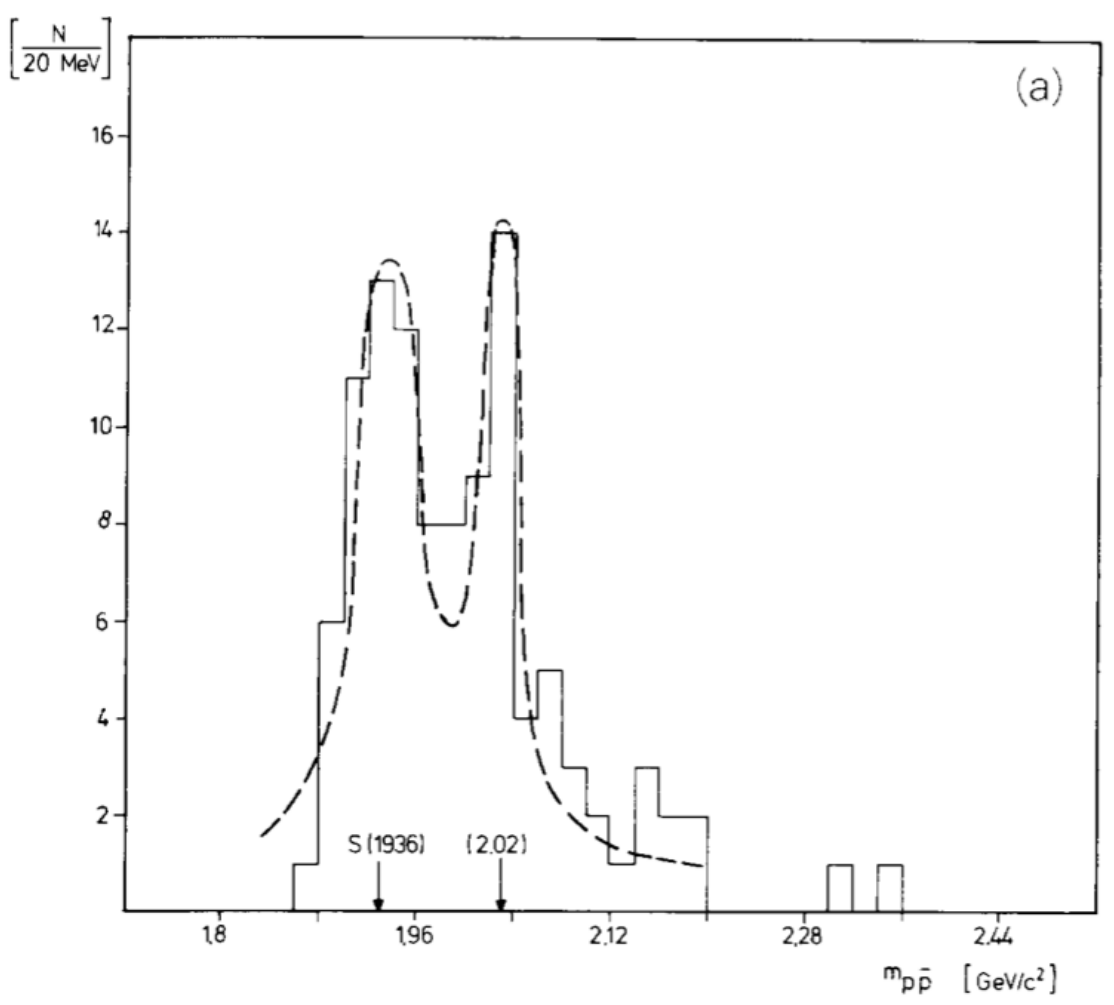

Figure 1.4: The invariant mass distributions for $p \bar{p}$ in the photoproduction reaction $\gamma p \rightarrow p p \bar{p}$ [8]. This distribution suggests a possible $2.02 \mathrm{GeV}^{2}$ resonance in photoproduction.

periments there have been intermediate states with low $(2 \mathrm{GeV})$ masses that are wide, most with widths between 200 and $400 \mathrm{MeV}$ such as the $f_{2}(2150), \rho(2150)$ and $f_{4}(2300)$, just to name a few [12]. These intermediate states have been observed decaying to $K K$ and $\pi \pi$ final states.

\subsubsection{Recent Work}

Since the beginning of the new millennium there have been analyses searching for baryon-antibaryon resonances in several experiments including CLAS at Jefferson Lab and BESIII. The earliest of these higher statistics works took place in 1999 with the CLAS g6b dataset with a total of 2,500 events. A hint of the narrow 


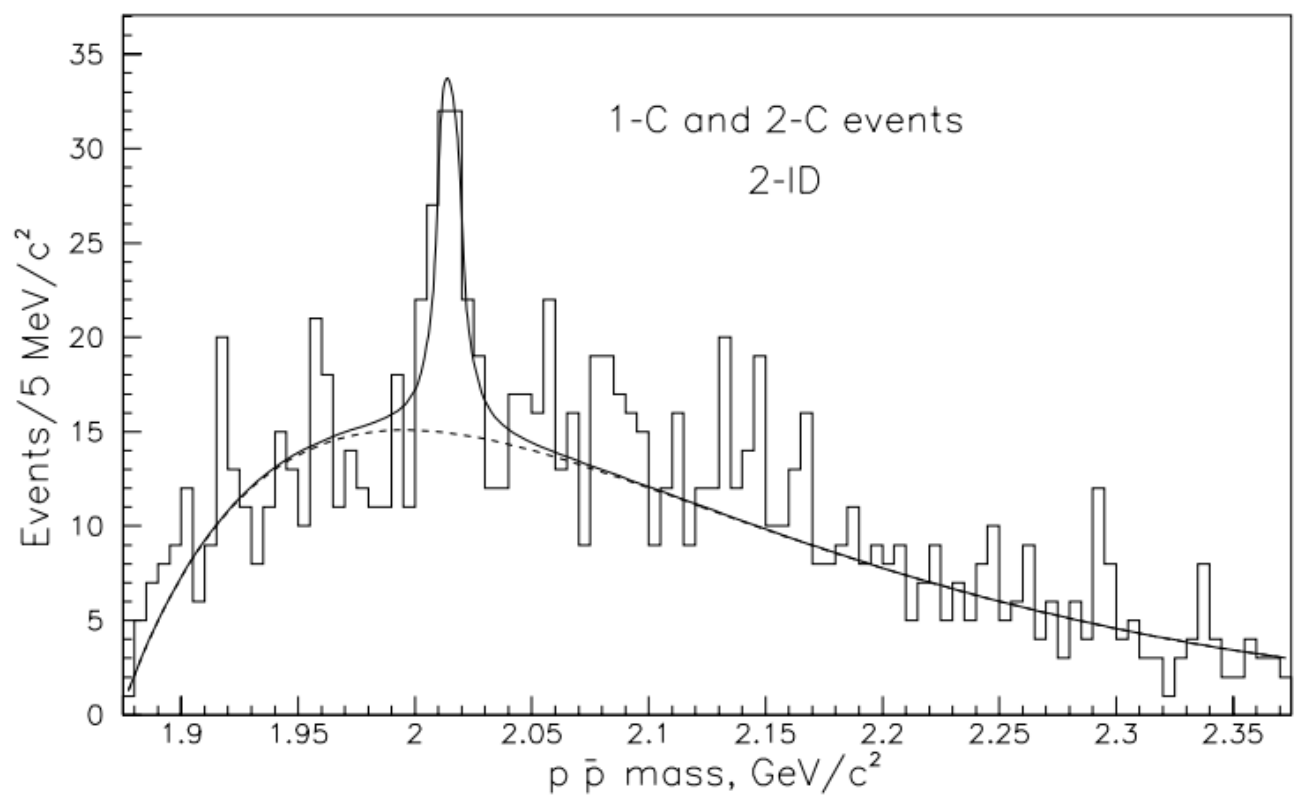

Figure 1.5: The invariant mass distributions for $p \bar{p}$ using a combination of all 3 and 4 prong datasets that were studied in the reanalysis of WA56 data in pion production [9].

resonance states observed in previous experiments is shown in Fig. 1.6 [13]. A total cross section from near-threshold beam energy to $5.3 \mathrm{GeV}$ was measured. The analysis is documented in an internal CLAS-Note. The renewed interest is a result of the availability of higher statistics data that was not previously available.

With an order of magnitude more data an analysis using CLAS g6c data, that was collected in 2001, did not observe narrow resonances (Fig. 1.7) [11]. A detailed moments analysis and a total cross section from a photon beam energy range of $4.9 \mathrm{GeV}$ to $5.5 \mathrm{GeV}$ was measured but was left unpublished.

In BESIII, there have been reports of a sub-threshold resonance, $X(1835)$ in the mass spectrum of $p \bar{p}$ in the reaction $e^{+} e^{-} \rightarrow J / \Psi$ where $J / \Psi \rightarrow p \bar{p} \gamma$ Fig. 1.8 , 


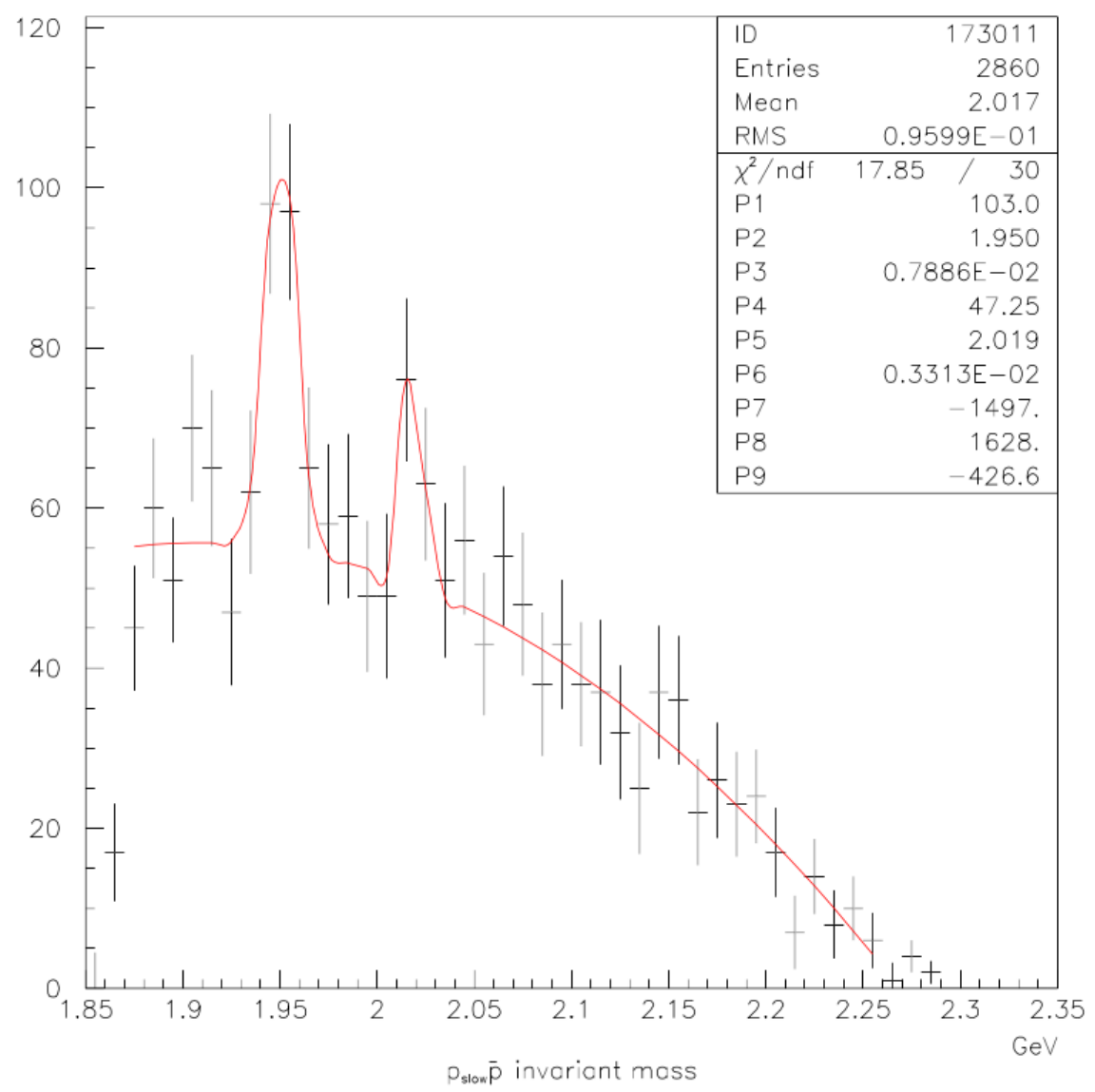

Figure 1.6: Evidence for narrow resonances from an unpublished analysis note in the CLAS collaboration, from the G6b run. [13]

\subsubsection{Theoretical Developments}

Spurred on by the development of high-intensity experiments such as GlueX, theoretical work by Lyubovitskij et al. has been published on the reaction $\gamma p \rightarrow$ $p p \bar{p}$ [14]. Lyubovitskij suggests that $p \bar{p}$ photoproduction would provide a less complicated means to search for a glueball or glueball components in $G \rightarrow$ $p \bar{p}$. The model uses effective hadronic Lagrangian with subthreshold resonances $f_{0}(1370), f_{0}(1500)$, and $f_{0}(1710)$ as shown in Figure 1.9. The model also predicts total cross sections and differential cross sections (Fig. 1.10). 


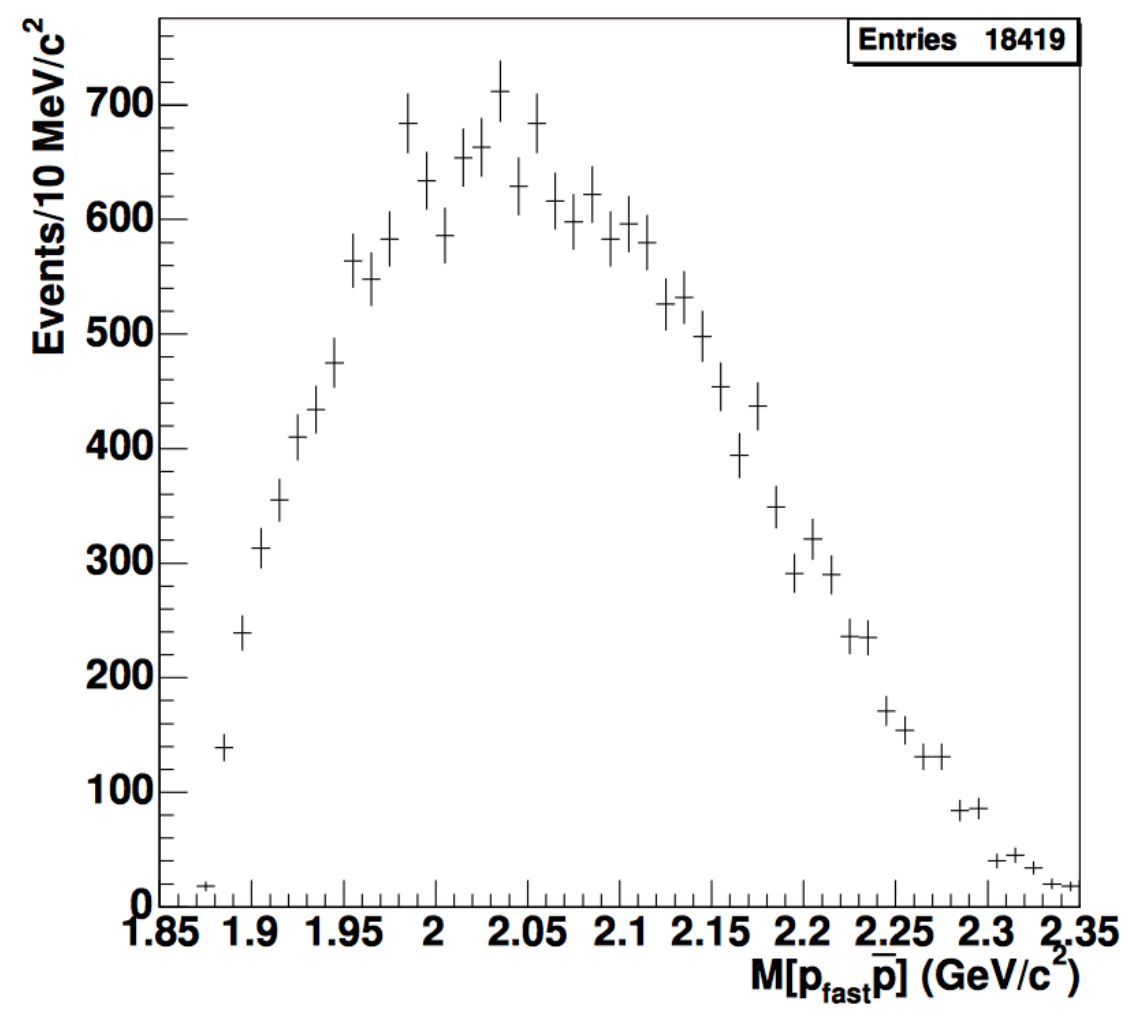

Figure 1.7: The invariant mass distribution of $p_{\text {fast }} \bar{p}$ showing statistically significant structure from a previous CLAS experiment, g6c. [11]

\subsubsection{Summary}

There have been many studies throughout the years on $p \bar{p}$ production using various reactions that have left us with many questions. This work seeks to describe the photoproduction mechanism of $p \bar{p}$ through measuring the first detailed differential cross sections and a total cross section from threshold to 5.45 $\mathrm{GeV}$ using the CLAS spectrometer at Jefferson Lab, which will be detailed in Chapter 2. The event selection and data processing will be discussed in Chapter 3. Cross section measurements for $\gamma p \rightarrow p p \bar{p}$ will be discussed in Chapter 4 and for $\gamma p \rightarrow p p \bar{n} \pi^{-}$in Chapter 5. Conclusions about the data and the future outlook will be discussed in Chapter 6 . 


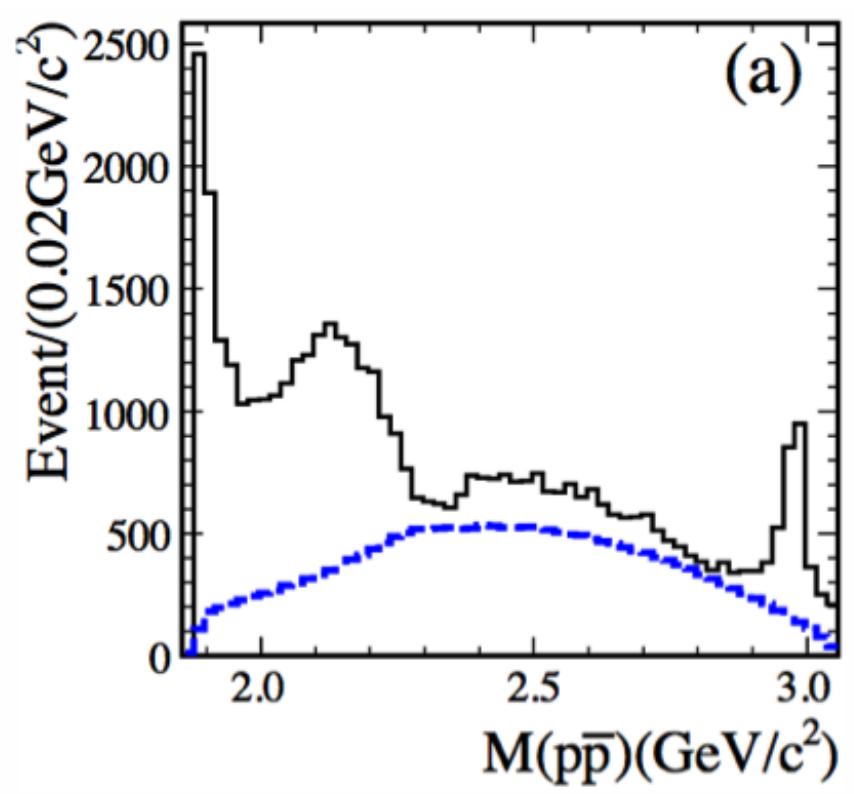

Figure 1.8: Resonances seen in the $p \bar{p}$ mass spectrum from BESIII in the reaction $J / \Psi \rightarrow p \bar{p} \gamma$. [6]

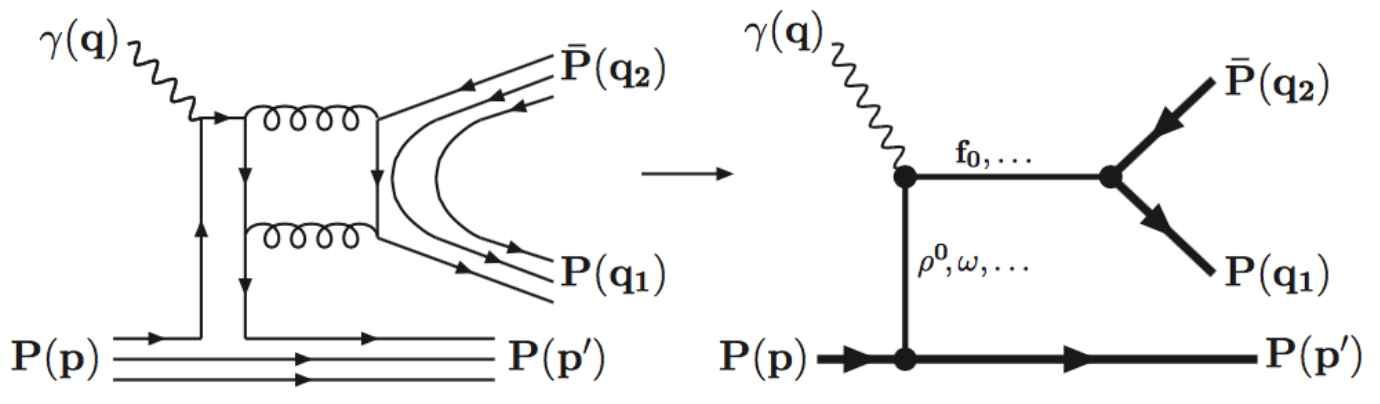

Figure 1.9: Feynman diagrams for $p \bar{p}$ photoproduction used for the calculation of the predictions in [14] 


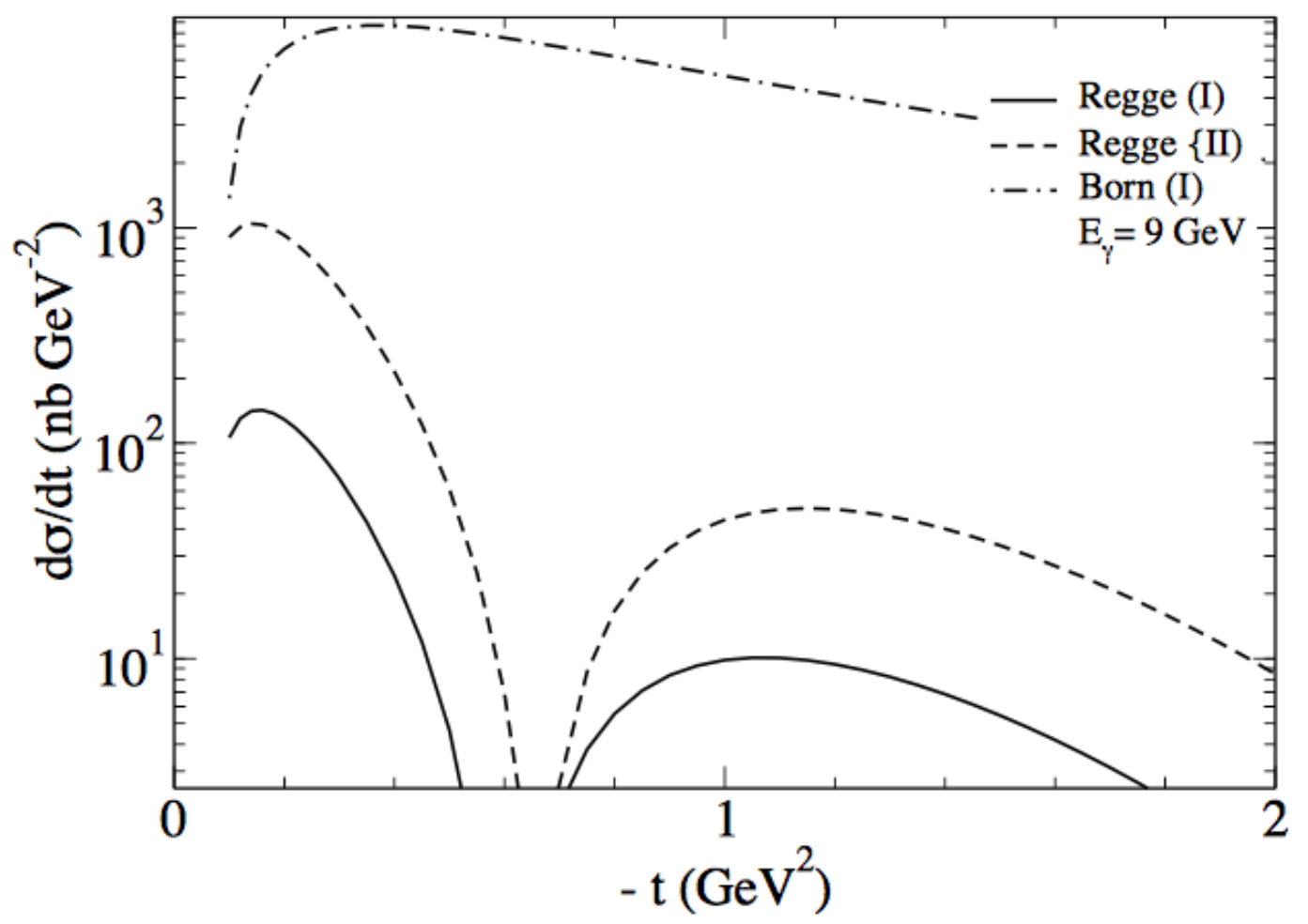

Figure 1.10: Predictions for differential cross section $d \sigma / d t$ from several different models at GlueX energies $\left(E_{\gamma}=9 \mathrm{GeV}\right)[14]$. 


\section{CHAPTER 2}

\section{Experimental Apparatus}

The data for this work were collected at the Thomas Jefferson National Accelerator Facility (Jefferson Lab). At the time of data collection, Jefferson Lab had the Continuous Electron Beam Accelerator Facility (CEBAF) accelerator and three

experimental halls A, B, and C (Fig. 2.1). The CEBAF Large Acceptance Spectrometer (CLAS) in Hall-B is comprised of many detector components to provide good charged particle resolution and a large angular acceptance. For this exper-

iment, a $10^{-4}$ radiation lengths thick gold radiator to produce a Bremsstrahlung photon beam and that was tagged to determine the photon beam energy. The data analyzed were from the $g 12$ rungroup, a photoproduction experiment with high incident flux on a $40 \mathrm{~cm} \mathrm{LH}_{2}$ target, with the purpose of searching for new forms of hadronic matter such as exotic mesons, pentaquarks, etc [15]. The same dataset is also ideal for antibaryon photoproduction due to the high luminosity.

\subsection{Accelerator}

\subsubsection{Overview of the Accelerator}

The CEBAF accelerator at the Thomas Jefferson National Accelerator Facility (Jefferson Lab) is a continuous electron beam accelerator (Fig. 2.2). The accelerator is in a racetrack configuration where two superconducting linear accel- 


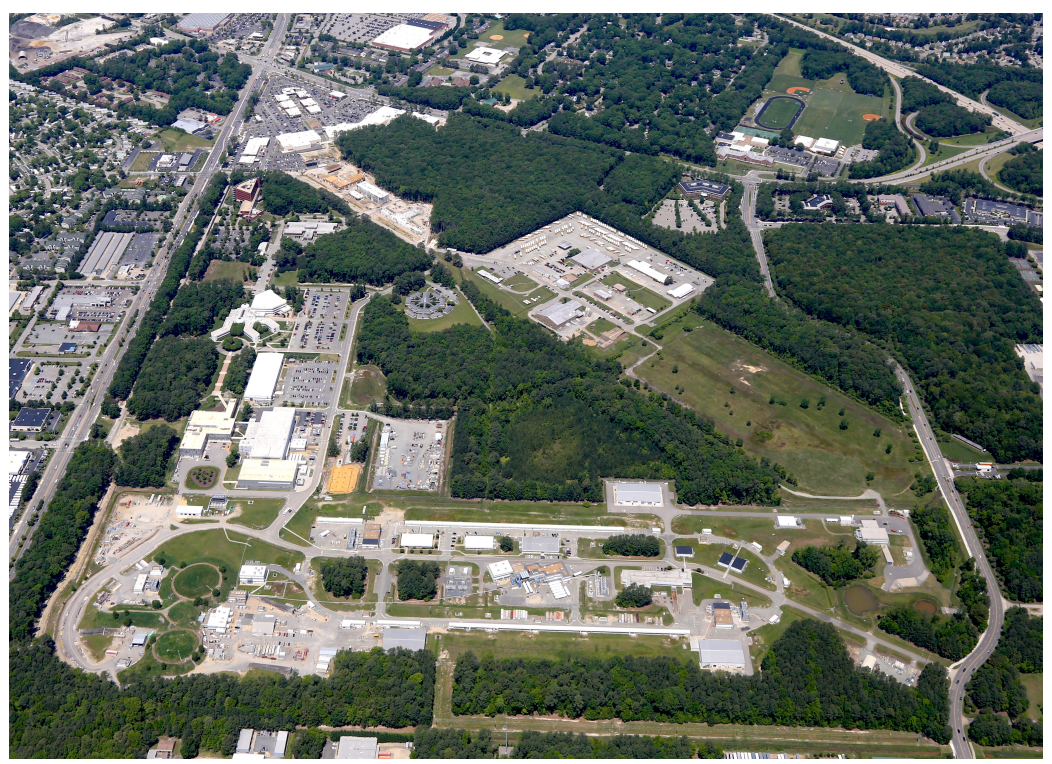

Figure 2.1: Jefferson Lab site aerial photo from May 2016 showing the CEBAF Accelerator, Engineering Buildings and office buildings. This photo shows the original three experimental halls at the lower left and the newest addition, Hall$\mathrm{D}$, at the lower right. The data for this work were taken in Hall-B, at the center of the original three halls.

erators form the straight sections that are connected by recirculation arcs. The $6 \mathrm{GeV}$ maximum electron beam requires the beam to be recirculated five times before finally reaching the beam splitter that distributes the beam to the three experimental halls (Hall A, B, and C). The accelerator was the first large-scale deployment of Superconducting Radio-Frequency cavities. The name CEBAF is actually slight misnomer since it is not truly continuous; rather, it operates at $500 \mathrm{MHz}$ with beam bunches arriving in 2 ns intervals.

The available electron beam energy range is from 0.8 to $6.0 \mathrm{GeV}$, with up to $85 \%$ polarization and a maximum luminosity of $10^{38} \mathrm{~cm}^{-2} \mathrm{~s}^{-1}$ in Halls $\mathrm{A}$ and C. However in Hall B the luminosity is required to be several orders of magnitude lower in order not to saturate the data acquisition system. 


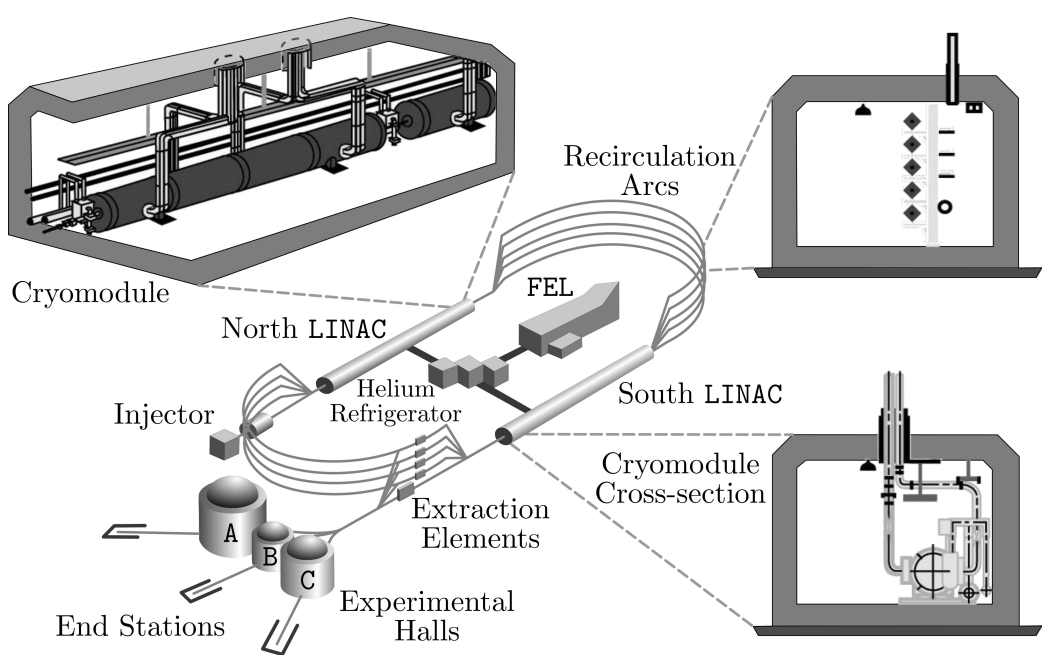

Figure 2.2: The CEBAF Accelerator overview. Showing the construction of the tunnels, injector, two linacs with the cryomodules, and the recirculating arcs with bending magnets.

\subsubsection{Superconducting Radio-Frequency Cavities}

The Superconducting Radio-Frequency (SRF) cavities are used to generate and direct the electric field that accelerates the electron beam bunches (Figs. 2.3 and 2.4). The cavities are constructed from pure niobium to allow for superconducting operation.The niobium blanks are pressed into half shells and then electronbeam welded to reduce defects. Once they have been assembled they are then grouped into sets of five for installation in cryomodules.

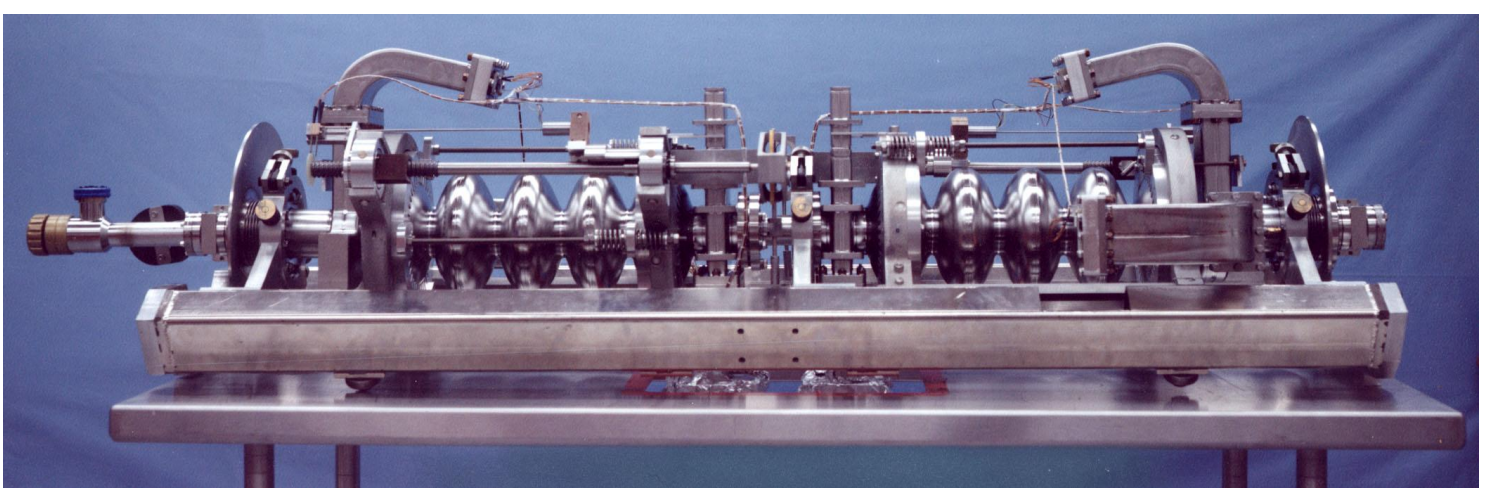

Figure 2.3: A pair of niobium cavities. 


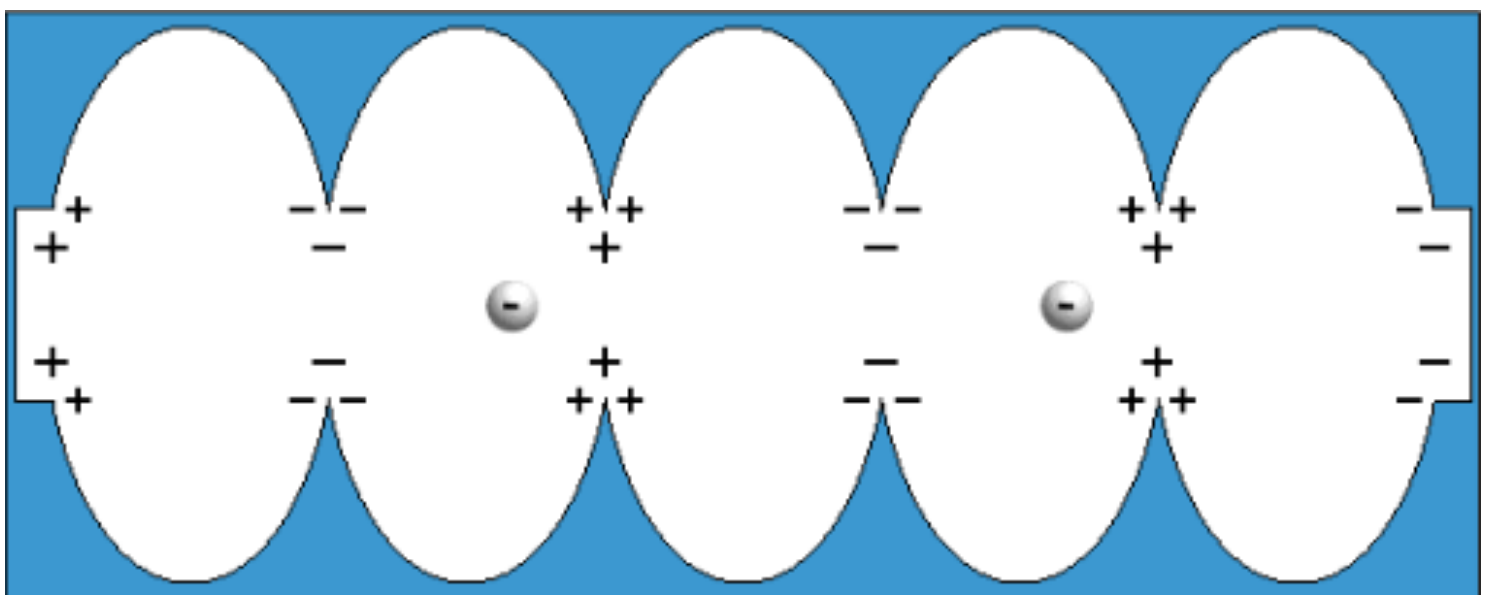

Figure 2.4: A SRF cavity showing the charge on the inner surfaces from the standing wave. This potential difference accelerates the electrons.

\subsection{Tagger and Beamline}

When experiments using CLAS operate with photon beams, electrons are incident upon a radiator to produce a bremsstrahlung photon beam [16]. The photon tagger is used to measure the energy of the photons by measuring the momentum of the scattered electron. The photon tagger in CLAS consists of a dipole magnet and 384 scintillating paddles that overlap, providing 767 logical paddles. These 767 logical paddles provide a photon energy resolution of $0.001 \cdot E_{0}$, where $E_{0}$ is the energy of the incident electron beam. The tagger is capable of tagging photons with energies of $20 \%$ to $95 \%$ of the incident electron energy. The overlapping rows of 767 logical paddles are referred to as the $E$ counters, as they are used to provide an accurate beam-energy measurement. In addition, there is a secondary row of tagger paddles below the $E$ counters, these are the $T$ counters. The $T$ counters consist of 61 scintillating paddles with a timing resolution of $110 \mathrm{ps}$, providing a more accurate timing resolution than the $E$ 
Counters. The $T$ counters are used to match the electron with the electron-beam RF bucket. The geometry is shown in Figure 2.5.

The difference between the incident electron energy, $E_{e^{-}}^{i}$, and the final electron energy, $E_{e^{-}}^{f}$, determine the photon energy

$$
E_{\gamma}=E_{e^{-}}^{i}-E_{e^{-}}^{f}
$$

The momentum transferred to the gold nucleus is negligible due to the mass of a gold nucleus which is significantly greater than the scattered electron.

In order to determine the photon flux several detectors are used. One is the total absorption shower calorimeter (TASC), which is a nearly $100 \%$ efficient lead glass crystal. As a consequence of photon pile up in the TASC the maximum beam current is limited to $100 \mathrm{pA}$. During normal beam operations the TASC is retracted because of the beam current limitations. The pair spectrometer is used for higher beam current operations and consists of a dipole magnet, a thin radiator, and scintillating paddles. The pair spectrometer works by measuring the rate of $e^{+} e^{-}$pair production that the magnet sweeps away from the beam and into the scintillating paddles. The pair spectrometer is located upstream of the target and is accurate in measuring the beam current to a few percent. The pair spectrometer is calibrated using information from the TASC. Additionally, for photoproduction experiments, out of time photons can be used in conjunction with the pair spectrometer and TASC to calculate the photon flux. The photon flux derived from the out of time photon information and the TASC is used heavily in this work as a normalization factor in order to calculate cross sections. 


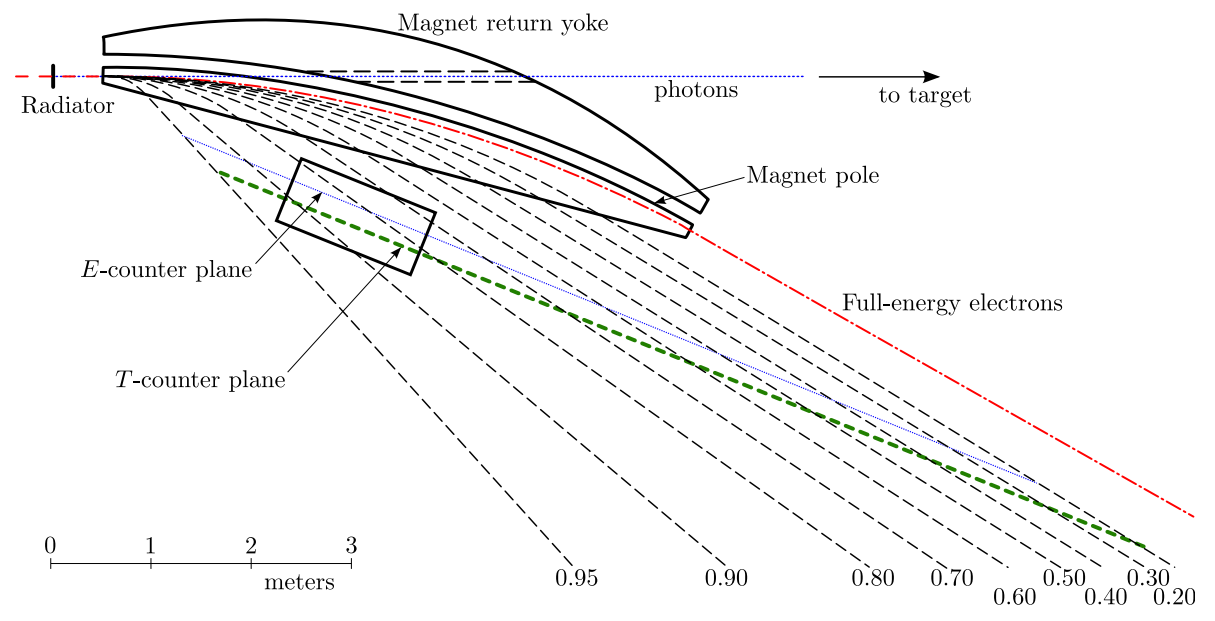

Figure 2.5: CLAS photon tagger. After electrons interact with a radiator and create bremsstrahlung photons they are bent into the scintillating paddles giving precise information about the timing and energy of the recoil electrons with energies $20-95 \%$ of the incident electron beam energy.

\subsection{CLAS Detector}

The CEBAF Large Acceptance Spectrometer (CLAS) is a large acceptance spectrometer that was designed for good charged particle momentum resolution and coverage to large angles. The specification was realized in the form of a toroidmagnet-based, large-acceptance detector design (Figs. 2.6 and 2.7). The torus magnet, drift chambers, start counter, and time-of-flight counters were used extensively in this analysis and will be described in the following sections. The electromagnetic calorimeter and Cherenkov counter were not used in this analysis but will be described for completeness. 


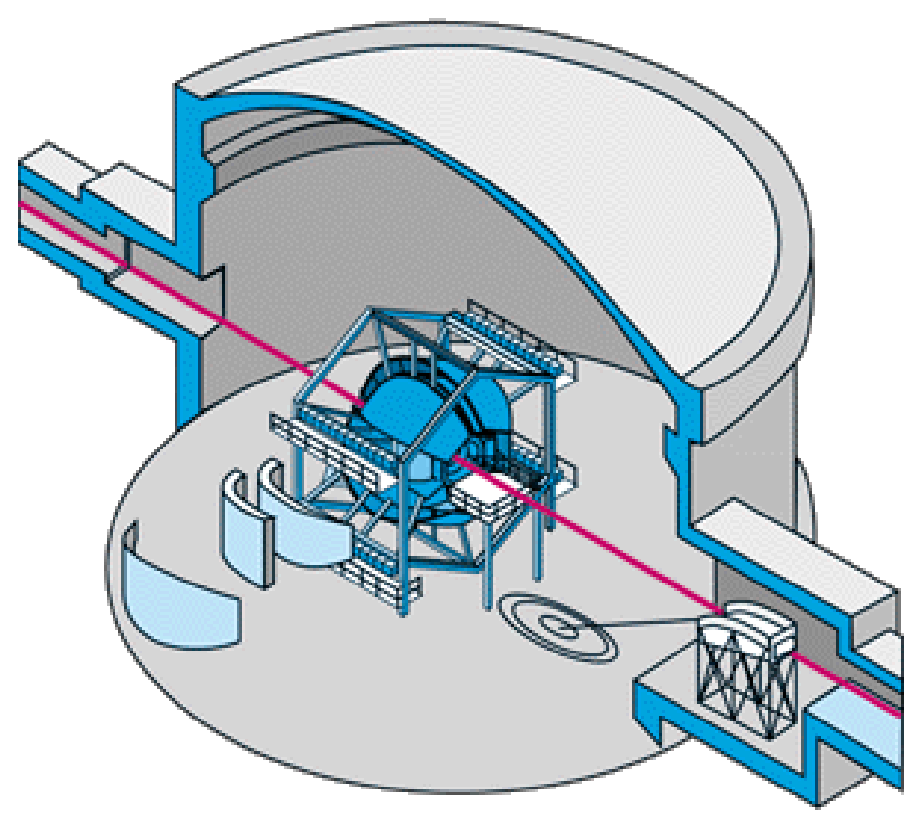

Figure 2.6: Three-dimensional model of Hall-B showing one of the six sectors in an exploded view with the CLAS detector shown in the center of the hall. The beam, shown in red, enters the hall from the lower right and passes through to the upper left.

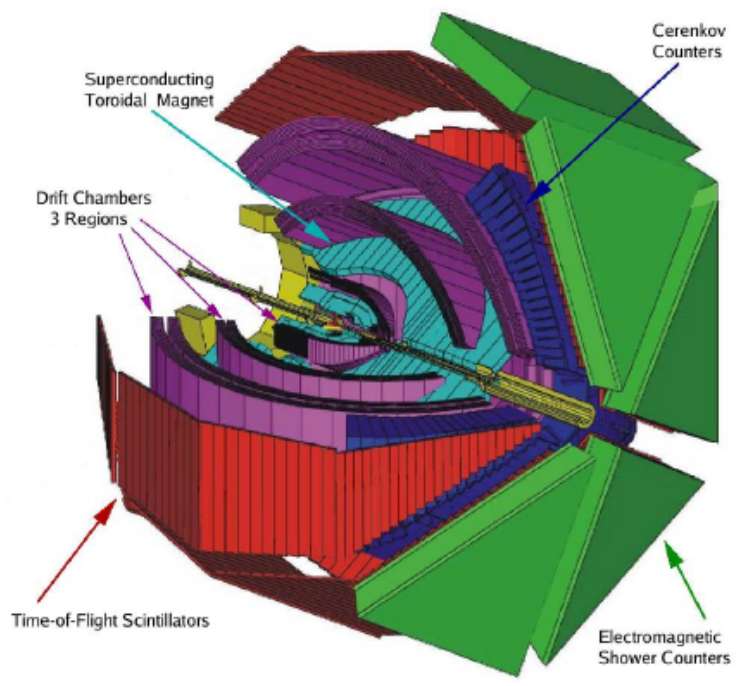

Figure 2.7: The CEBAF Large Acceptance Spectrometer (CLAS). The orientation of the figure has the beam entering from the upper left and exits to the beam dump on the lower right. 


\subsubsection{Torus}

The purpose of the superconducting torus magnet is to bend charged particles in the magnetic field to determine their charge and momentum in combination with other detector subsystems. The torus magnet was the key aspect of the detector design about which every other detector subsystem was designed. The design consists of six kidney-shaped magnets that are evenly distributed azimuthally, producing a magnetic field that is approximately toroidal (Fig. 2.8). The six-sector design drove the drift chamber development. The drift chambers are situated in between the torus coils and will be discussed further in Sec. 3.3.3. The maximum current is $3860 \mathrm{~A}$, however the field used during the $g_{12}$ run period is half of the maximum current (1930A), which corresponds to the field shown in (Fig. 2.9).

The coils themselves are made of aluminum-stabilized $\mathrm{NbTi} / \mathrm{Cu}$ conductor surrounded by a vacuum jacket. The coils are attached to the cryostat by three support links. An important design consideration is that these links will support tremendous forces due to inter-coil forces that can be caused by coil misalignment or defects.

\subsubsection{Start Counter}

The upgraded Start Counter is used in the trigger and in conjunction with the TOF and the photon tagger information to determine the beam bunch of the event $[17]$. The new highly-segmented start counter is a major improvement on the old start counter having 24 channels instead of 3 to keep electromagnetic background down by requiring at least two paddles be hit (Fig. 2.10). The goal of the new start counter was to allow for increased luminosity running through 


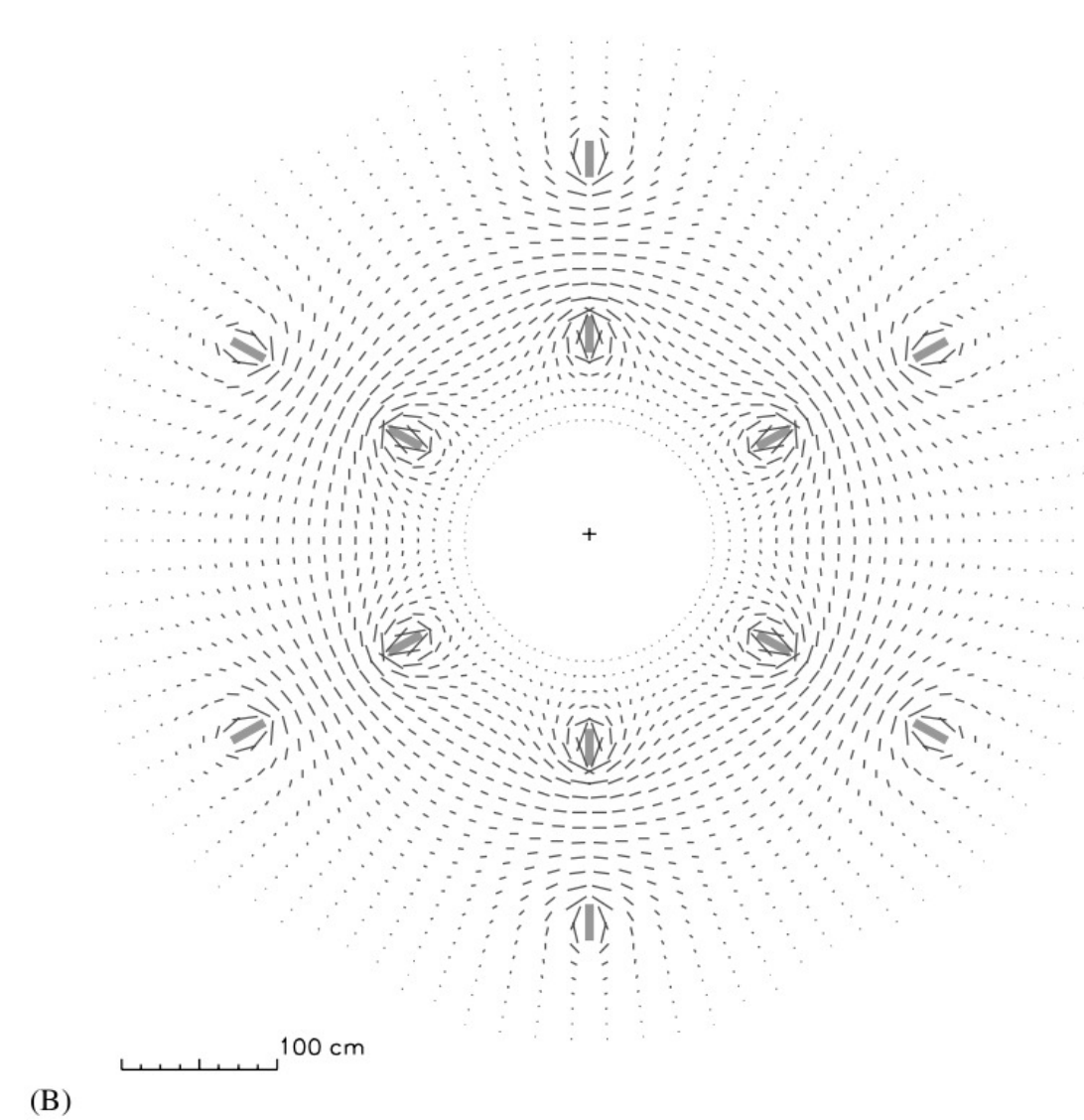

Figure 2.8: The torus field lines looking downstream at the midplane of CLAS. It is important to note that the major component of the field lines is in the azimuthal direction.

accommodating a longer target and with the increased number of paddles the Photomultiplier Tubes (PMTs) were no longer saturated.

The design consists of six sectors containing four EJ-200 scintillators per sector. Each paddle was bent near the nose region and then machined to form a nearly hermetic seal with the other paddles. These scintillating paddles are read out by 24 Hammamatsu R4125HA PMTs and have an overall resolution of 388 ps. 


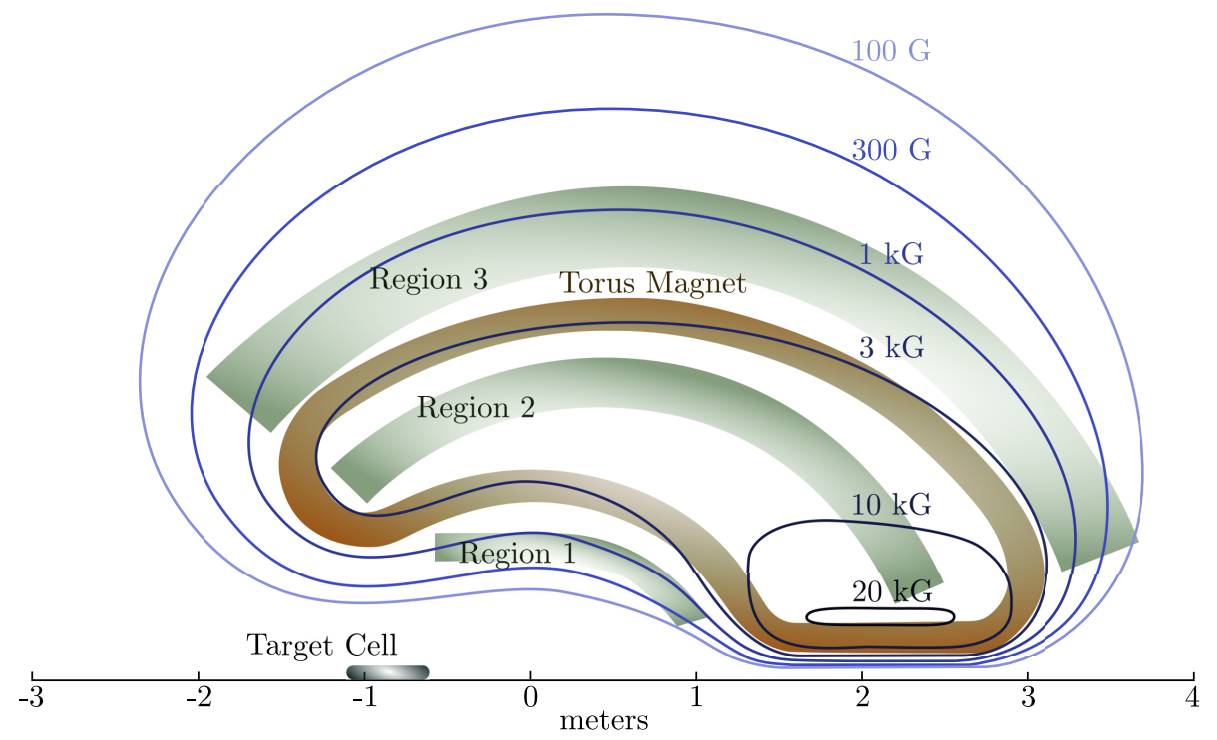

Figure 2.9: The CLAS torus field map, parallel to the beamline, at half of the max production current. Also shown is the target placed at $-90 \mathrm{~cm}$ from CLAS center.

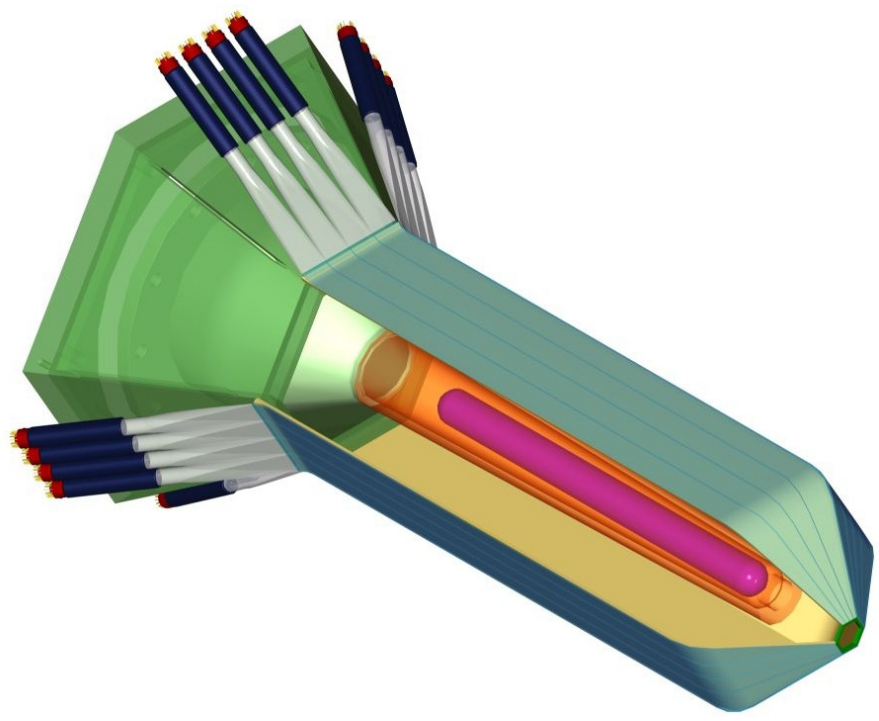

Figure 2.10: The upgraded, highly-segmented CLAS Start counter. Inside the start counter the $40 \mathrm{~cm}$ long target cell is visible.[17] 


\subsubsection{Drift Chambers}

The CLAS drift chambers are used for determining the charge and momentum of charged particles (Fig. 2.11) [18]. The drift chamber design was driven by the torus magnet and is situated between the six kidney-shaped coils. There are three "regions" located in each of the six sectors; region 1 closest to the target, region 2 within the strongest magnetic field, and region 3 further out from the target.

The drift chambers are comprised of many thousands of wires that are separated into two types, the guard and sense wires. There are six guard wires that form each hexagonal cell with a sense wire at the center. The guard wires and sense wires have a high voltage applied to them creating an electric field that points radially outward from the sense wire. When charged particles pass through the cell the gas is ionized within the cell and the electric field causes the ions to drift towards the sense wire. The resulting avalanche of ions is detected and the time is recorded allowing for a more precise determination of the position of the track within the cell.

The three regions of drift chambers are comprised of two "superlayers." These superlayers are at a $6^{\circ}$ angle to each other in order to provide azimuthal angle information. Inside each of the superlayers are six layers of hexagonally shaped cells, with the exception being the first super layer in sector one, which contained four layers due to space constraints. These hexagonal cells are bordered by goldplated aluminum field wires and at the center, a single gold-plated tungsten sense wire. Guard wires shape the field at the outer layers of the cells. In total, there are 35,148 cells that range in size from $0.7 \mathrm{~cm}$ in Region $1,1.5 \mathrm{~cm}$ in Region 2 , to $2.0 \mathrm{~cm}$ in Region 3. 


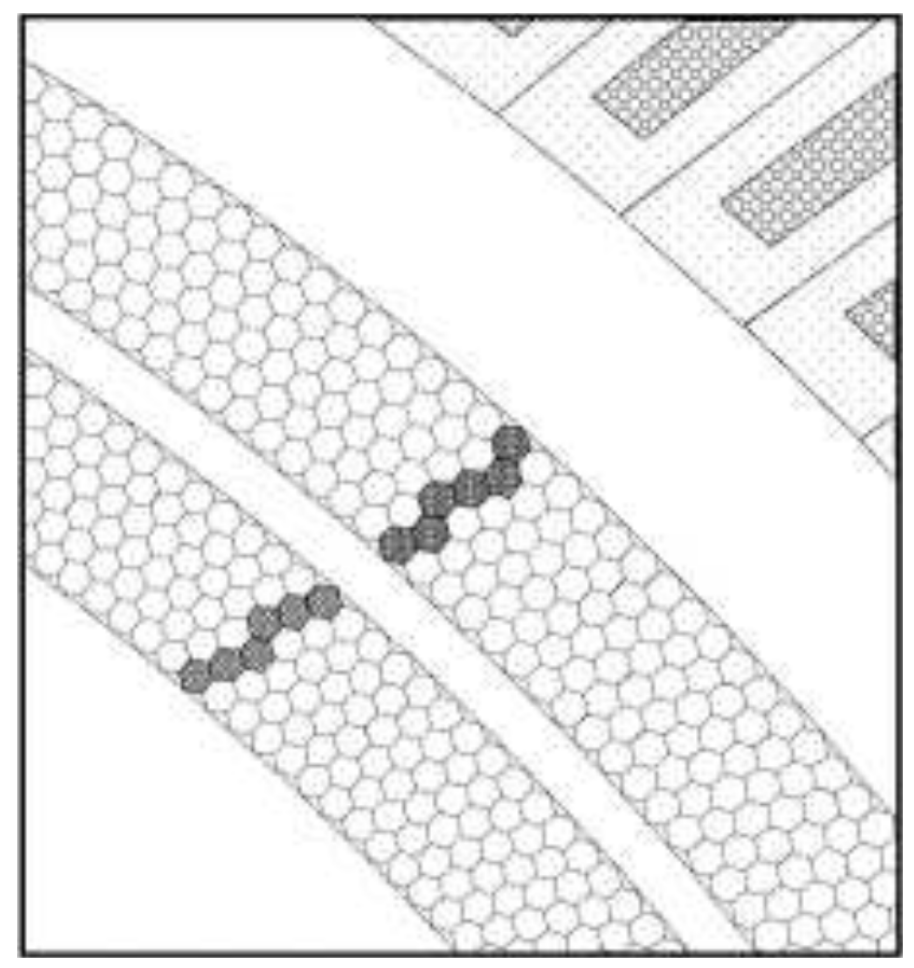

Figure 2.11: A CLAS Event Display view of a track going through two superlayers of the drift chambers.

\subsubsection{Time-of-Flight Counters}

The Time-of-Flight (TOF) counters are used in conjunction with flight path information for particle identification and are also used in the trigger (Fig. 2.12) [19]. They were specifically designed to provide a large signal for minimum ionizing particles. The angular range covered by the counters is from $8^{\circ}$ to $142^{\circ}$ in polar angle and $360^{\circ}$ in the azimuthal range. The bars are made from Bicron BC-408 scintillators with Thorn EMI 9954A PMTs at forward angles and Phillips 4312B/D2 at large angles. Each bar has two PMTs total, one at each end. These scintillators are $5.08 \mathrm{~cm}$ thick and vary in length from 32 to $375 \mathrm{~cm}$ which yields a resolution ranging from 75 to 160 ps. 


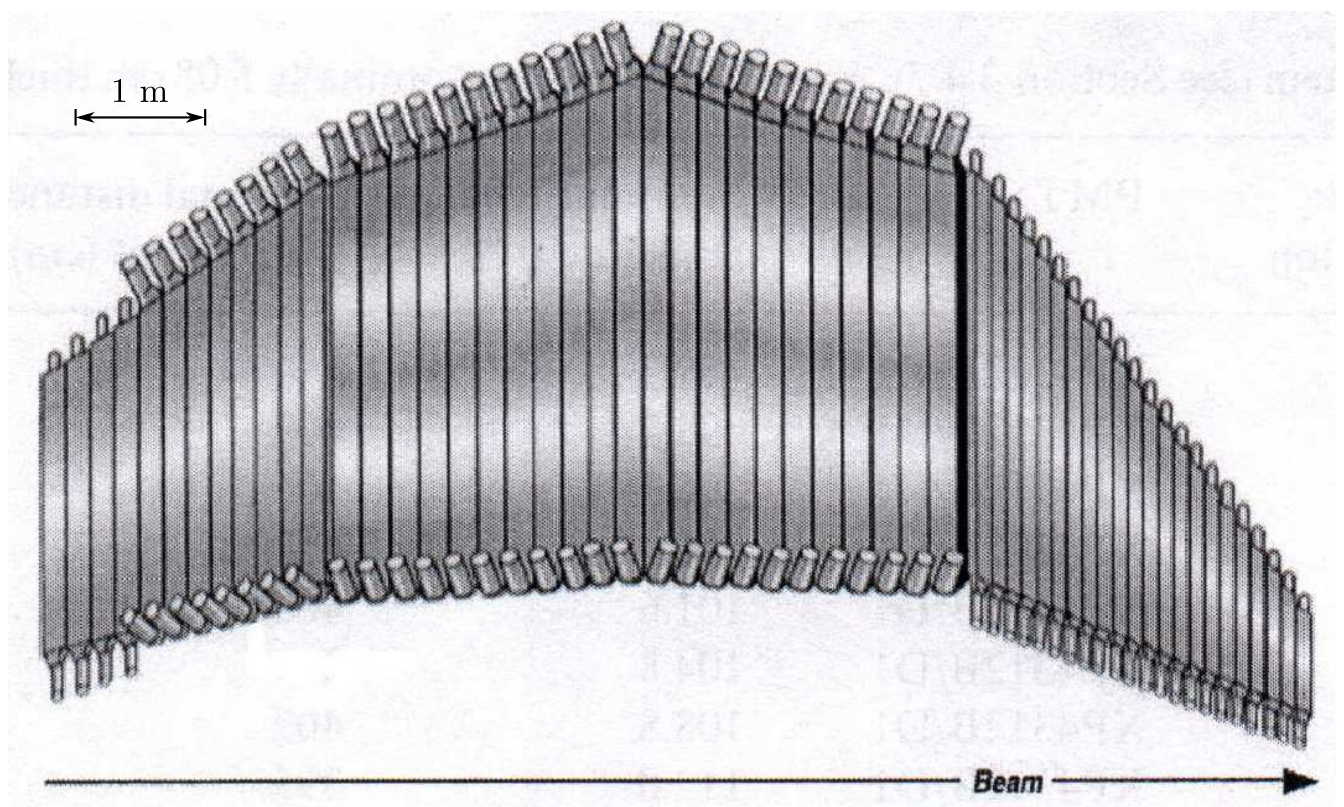

Figure 2.12: The 57 Time of Flight (TOF) scintillating paddles for one sector [19].

\subsubsection{Cherenkov Counter}

The CLAS Cherenkov Counter was used for separating electrons and pions [20]. Cherenkov counters work on the principle of Cherenkov radiation: when a charged particle exceeds the speed of light in a dielectric medium the medium emits photons as the particle passes through it. The radiator gas chosen for this detector is $\mathrm{C}_{4} \mathrm{~F}_{10}$, giving a pion momentum threshold of $2.5 \mathrm{GeV} / \mathrm{c}^{2}$. The counters extend to a maximum polar angle of $45^{\circ}$, and cover nearly the entire azimuthal range.

The design of the Cherenkov Counter consists of 6 volumes, one for each sector. Inside each volume there are two rows containing 18 mirror facets and light collection devices each for a total of 216 PMTs. The diagram shown in Fig. 2.13 shows the three different mirror facets used to collect light; elliptical, hyperbolic and cylindrical geometries are used. 


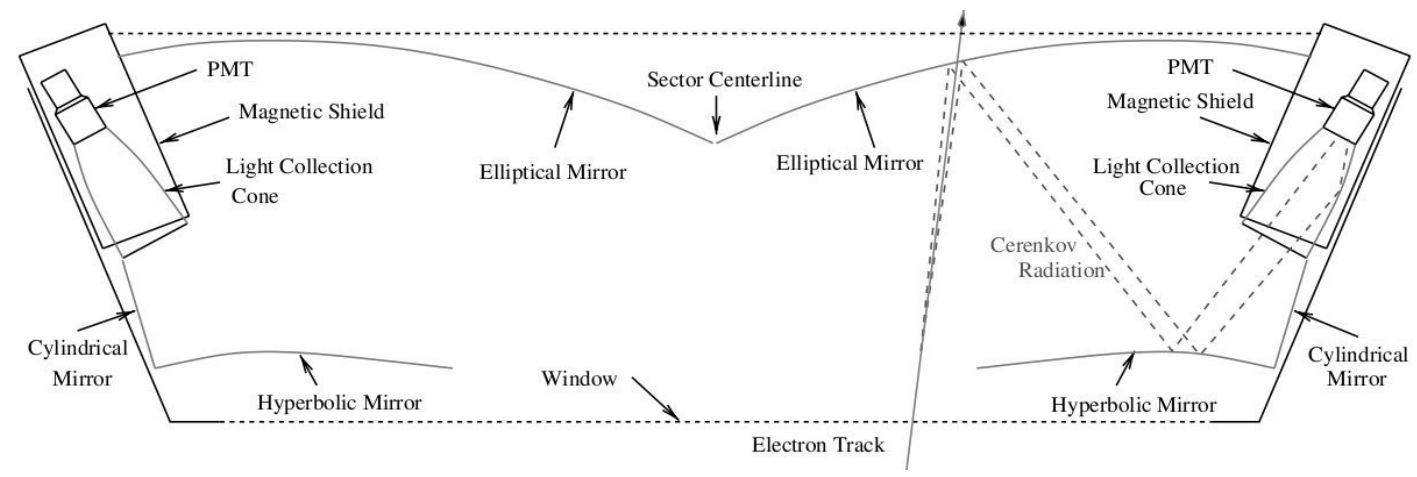

Figure 2.13: Cross-sectional view of a Cherenkov Counter sector. The track that is shown is an electron collection trajectory [20].

\subsubsection{Electromagnetic Calorimeter}

The electromagnetic calorimeter (Fig. 2.14) was used for detection of electrons above $0.5 \mathrm{GeV}$ and for photons above $0.2 \mathrm{GeV}$ [21]. While it is primarily used for reconstructing $\pi^{0}$ and $\eta$ radiative decays, it can also be used for leptons and neutral particles. The design consists of 39 layers alternating between 2.2-mmthick lead sheets and 10-mm-thick scintillator bars. The scintillators are arranged to form three "views", called $U, V$, and $W$, which are offset by 120 degrees so that the electromagnetic showers can be spatially located by the pixels created with the overlapping layers. The readout of each $U, V$, and $W$ orientation is done by dividing the calorimeter into two parts, an inner layer comprised of the first 5 layers and the outer 8 layers. In total, for all six sectors, 1296 XP2262 PMTs are used. 


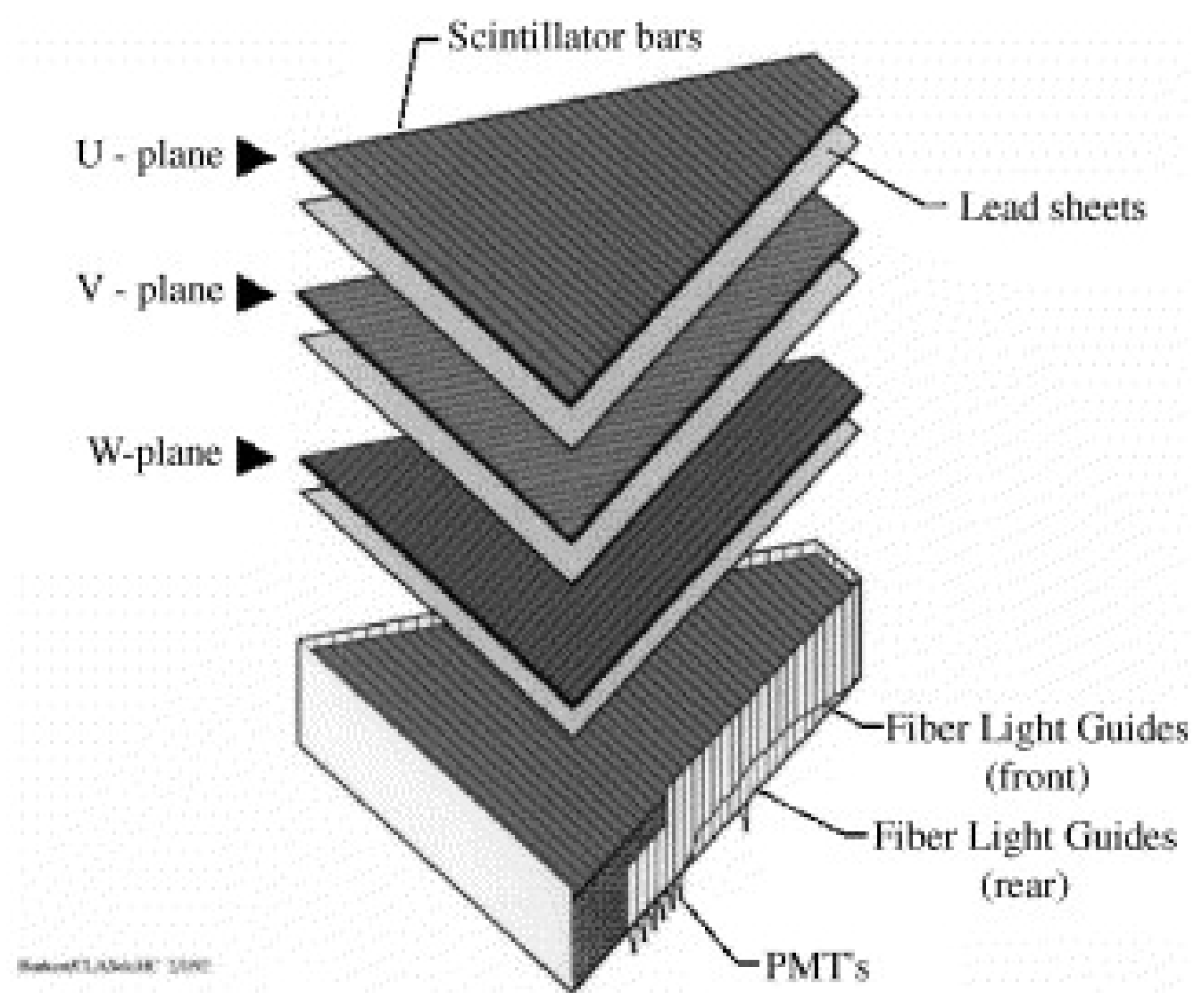

Figure 2.14: One sector of the electromagnetic calorimeter exploded to show the internal construction and arrangement of the layers [22].

\subsection{Data Acquisition}

The unprocessed signals from the detector systems are read out by back-end electronics crates that convert the analog signals into digital signals. The modules that do this for signal amplitude are Analog to Digital Converters (ADCs), and those modules that are more sensitive to the signal rise or fall time are Time to Digital Converters (TDCs) for timing information.

In order to maximize the event rate for the type of physics events that are being studied, there is a trigger for recording the events to disk. The level 1 trigger was programmed into a Field Programmable Gate Array (FPGA) and 
Table 2.1: Trigger configuration for the majority of g12 production runs, specifically from 56595 to 56607 and 56648 to 57323 . MORA corresponds to a tagger energy greater than $4.4 \mathrm{GeV}$ and MORB corresponds to a tagger energy from 3.6 to $4.4 \mathrm{GeV}$.

\begin{tabular}{|c|c|c|c|}
\hline \multicolumn{4}{|c|}{$g_{12}$ runs $56595-56607,56648-57323$} \\
\hline bit & definition & L2 multiplicity $^{a}$ & prescale \\
\hline 1 & $\mathrm{MORA} \cdot(\mathrm{ST} \times \mathrm{TOF})$ & 1 & $1000 / 300^{b}$ \\
\hline 2 & $\mathrm{MORA} \cdot(\mathrm{ST} \times \mathrm{TOF}) \times 2$ & $2 /-^{C}$ & 1 \\
\hline 3 & $\mathrm{MORB} \cdot(\mathrm{ST} \times \mathrm{TOF}) \times 2$ & $2^{\square}$ & 1 \\
\hline 4 & $\mathrm{ST} \times \mathrm{TOF}$ & 1 & $1000 / 300$ \\
\hline 5 & $(\mathrm{ST} \times \mathrm{TOF}) \cdot \mathrm{ECP} \times 2$ & 1 & 1 \\
\hline 6 & $(\mathrm{ST} \times \mathrm{TOF}) \cdot(\mathrm{EC} \times \mathrm{CC})$ & 2 & 1 \\
\hline 7 & $\mathrm{MORA} \cdot(\mathrm{ST} \times \mathrm{TOF}) \cdot(\mathrm{EC} \times \mathrm{CC})$ & - & 1 \\
\hline 8 & MORA $\cdot(\mathrm{ST} \times \mathrm{TOF}) \times 2$ & - & 1 \\
\hline 11 & $(\mathrm{EC} \times \mathrm{CC}) \times 2$ & - & 1 \\
\hline 12 & $(\mathrm{ST} \times \mathrm{TOF}) \times 3$ & - & 1 \\
\hline
\end{tabular}

${ }^{a}$ Level 2 triggering was turned off on all bits for runs 56605, 56607 and 56647 .

${ }^{b}$ Prescaling for bits 1 and 4 were 1000 for runs prior to 56668 at which point they both were changed to 300 .

${ }^{c}$ Level 2 triggering of bit 2 was set to 2 for runs prior to 56665 at which point it was turned off.

originally had 9 bits that could be programmed. Table 2.1 shows the 12 different trigger bit configurations. In addition to the level 1 trigger, a level 2 trigger was used with a fast tracking algorithm to verify there was a track in the sector before writing out the event. 


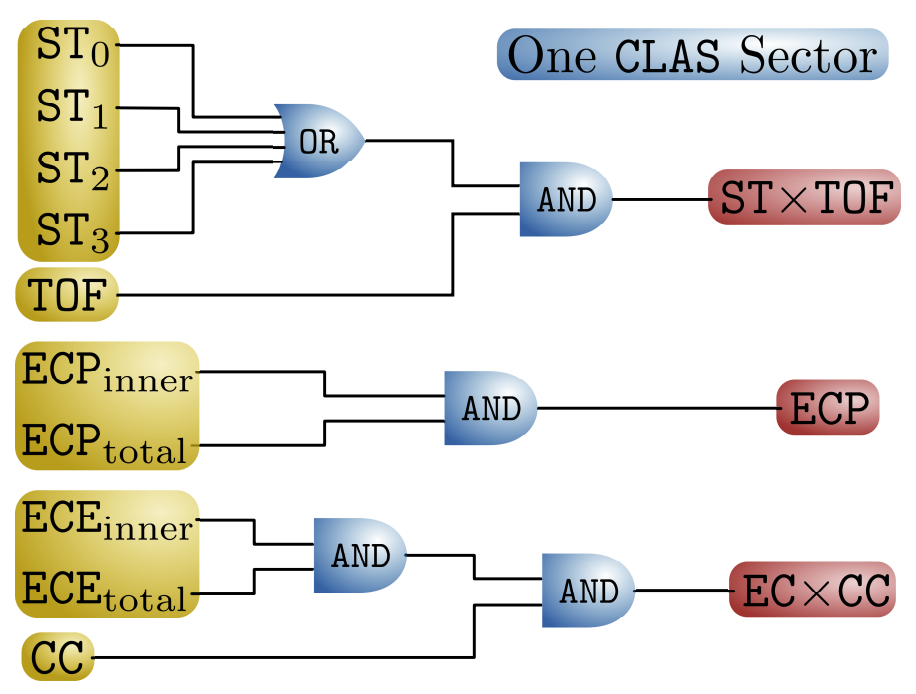

Figure 2.15: A diagram showing the trigger logic for one sector of CLAS. The $\mathrm{ST} \times \mathrm{TOF}$ can only determine if there was a single track in the trigger, so the multitrack trigger requirement requires the tracks to be in different sectors. The $\mathrm{EC} \times \mathrm{CC}$ trigger was used for Lepton identification.

\subsection{The $g_{12}$ experiment}

Experiment E-04-005 (g12) was a high statistics photoproduction experiment that ran from April until June in 2008 (Tab. 2.2). It was the highest statistics photoproduction experiment ever with an overall integrated luminosity of $68 \mathrm{pb}^{-1}$. The purpose of this experiment was to search for new forms of exotic hadronic matter. It was the highest statistics photoproduction experiment ever with an overall integrated luminosity of $68 \mathrm{pb}^{-1}$. A $40 \mathrm{~cm}$ long and $4 \mathrm{~cm}$ diameter $\ell \mathrm{H}_{2}$ target was placed $90 \mathrm{~cm}$ upstream of the standard target position for CLAS. The new target position increases the acceptance in the forward direction by reducing the hole in acceptance in the forward direction. The Bremsstrahlung photon energy range used in the current analysis was $3.95-5.45 \mathrm{GeV}$. In order to reach the required luminosity for this experiment a larger target and a higher beam current than previous photoproduction experiments in Hall B was needed. The 
g12 experiment provides the world's largest dataset on $p \bar{p}$ photoproduction at these energies. The trigger configuration for one sector is shown in Figure 2.15 and the experiment specifications are summarized in Table 2.2 .

Table 2.2: The running conditions of the $g 12$ experiment from [23]

\begin{tabular}{lc}
\hline \hline$E_{\text {beam }}$ of electron & $5 \cdot 715 \mathrm{GeV}$ \\
Beam Polarization & Circular \\
$\mathrm{e}^{-}$Current & $60-65 \mathrm{nA}$ \\
Tagger Range & $5 \%-95 \%$ of e $\mathrm{e}^{-}$energy \\
Tagger Trigger Range & $3.6-5 \cdot 441 \mathrm{GeV}$ \\
Torus Magnet & $\frac{1}{2} B_{\max }(1930 \mathrm{~A})$ \\
Target Length & $40 \mathrm{~cm}$ \\
Target Center $(z$ location $)$ & $-90 \mathrm{~cm}$ \\
Target Material & $\mathrm{LH} \mathrm{H}_{2}$ \\
Target Polarization & $\mathrm{None}$ \\
Start Counter Offset & $\mathrm{O} \mathrm{cm}$ \\
Radiator Thickness & $10^{-4}$ radiation lengths \\
Collimator Radius & $6.4 \mathrm{~mm}$ \\
\hline \hline
\end{tabular}

\subsubsection{Target}

The target that was used for this experiment is a $40 \mathrm{~cm}$ long $4 \mathrm{~cm}$ diameter Kapton target intended to provide higher luminosities through increased target length. For the $g_{12}$ experiment the target was filled with liquid hydrogen $\left(\ell \mathrm{H}_{2}\right)$. The target cell is constructed in a truncated conical shape to aid in the removal of hydrogen bubbles, this is shown in Figure 2.16 


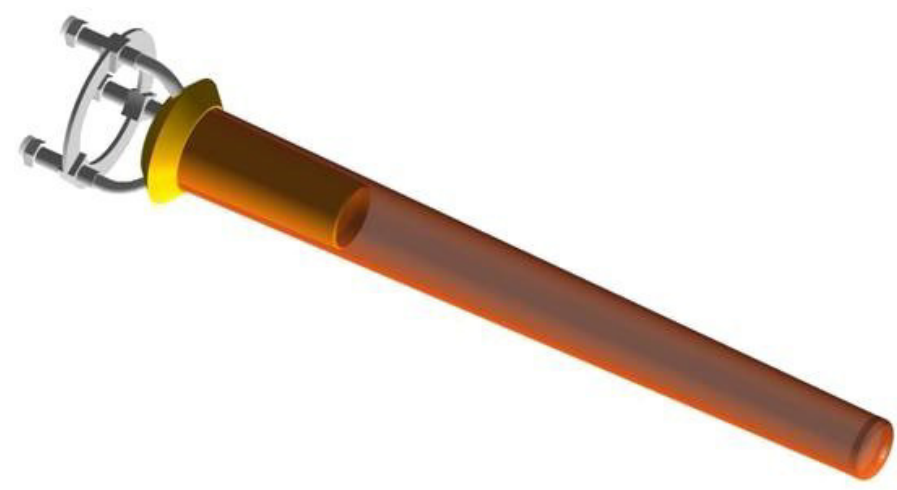

Figure 2.16: The gi1a 40-cm-long, 4-cm-diameter target cell, constructed in a truncated conical design from kapton.

\subsection{Event Reconstruction}

Charged particles scintillate in media, allowing the use of TOF counters, and they also ionize gas, allowing for the use the DC to determine the momentum and tracking information. In order to reconstruct a charged particle track, information from the drift chambers is used to determine the charge and momentum of the tracks when combined with the magnetic field information. Track reconstruction is done with a fit of a track to each of the 6 superlayers independently in order to first identify tracks. The information from the sense wire is the relative hit time, which can lead to an ambiguity often called the left-right ambiguity in which the track could be either on the left or right side of the sense wires. The ambiguity is resolved when performing an overall fit incorporating 5 of the 6 super layers. The fit uses 5 out of the 6 superlayers in order to reduce the effect of dead wires and regions. The individual superlayers have a detection efficiency of $98 \%$. The difference in time for the particles to travel from the start counter to the TOF determines the velocity. In combination with the momentum information from the drift chambers we can reconstruct the mass of the particles and use that for particle identification. Noting that $\beta=v / c$, and that the velocity of 
the particle is determined by the TOF and the momentum is determined from the $\mathrm{DC}$, the mass is calculated by

$$
m=\frac{p}{\beta \gamma c} .
$$

For neutral particles the electromagnetic calorimeter is used to determine the energy of the particles. The EC reconstruction uses the $U, V$, and $W$ layers of the $\mathrm{EC}$ in order to form pixels. These pixels are then grouped together to form a hit cluster allowing the energy of the hit to be summed. Photon energy is determined by the energy deposited in the EC and charged tracks and hits in the TOF are used to veto charged particles and reduce false tracks. Neutron detection involves the energy deposited in the EC as well as the timing information used to determine the $\beta$ of the particle and determine the momentum. 


\section{CHAPTER 3}

\section{Data Analysis}

The main focus of this analysis is the $\gamma p \rightarrow p p \bar{p}$ reaction. This chapter will discuss event selection, topologies that are used, and corrections to the data.

\subsection{Event Selection}

The topologies studied for the reaction $\gamma p \rightarrow p p \bar{p}$ have either two detected protons with a missing antiproton $(\gamma p \rightarrow p p(\bar{p}))$ or a detected proton and a detected antiproton with a missing proton $(\gamma p \rightarrow p(p) \bar{p})$. In the first topology the antiprotons are generally not detected since they were bent into the forward hole of CLAS. This topology required the reconstruction of the undetected antiproton through the missing mass shown in Figure 3.3.1. The topology with a missing proton required the reconstruction of the undetected proton through the missing mass and is shown in Figure 3.3.1. The events where all three final-state particles are reconstructed are included in the missing antiproton dataset.

\subsubsection{Data Selection Cuts}

In order to reduce the background of events other than $\gamma p \rightarrow p p \bar{p}$ some initial cuts must be made such as timing, event vertex, fiducial cuts, and limiting the number of reconstructed tracks other than protons and antiprotons. With all 


\begin{tabular}{c|c|c}
\hline Cut & Number of events & Signal/(Signal+Background) \\
\hline \hline No cuts & 291958 & 0.40 \\
Event timing & 290296 & 0.51 \\
Target Fiducial & 227069 & 0.64 \\
Detector Fiducial & 219384 & 0.65 \\
TOF Knock Out & 193364 & 0.66 \\
\hline
\end{tabular}

Table 3.1: List of cuts applied and the affect on the signal/(signal+background) ratio for the reaction $\gamma p \rightarrow p p(\bar{p})$.

\begin{tabular}{c|c}
\hline Topology & Number of Events \\
\hline \hline$p p(\bar{p})$ or $p p \bar{p}$ & 229117 \\
$p \bar{p}(p)$ & 79268 \\
\hline
\end{tabular}

Table 3.2: Yield of events for the two topologies used after all cuts were applied.

cuts applied the background is $34 \%$. Table 3.1 is a list of cuts and their effect on the signal to background ratio. Each cut is in combination with the previous cut from the line above, starting with no cuts other than a rough event selection of requiring that two protons are reconstructed.

The background could be further reduced with restricting the number of particles being reconstructed in the final state, however this can bias the measurements as it is not accurately accounted for in simulation. In the forward direction there are neutral particles that are ghost tracks or are reconstructed but are out of time.

\subsubsection{Target Fiducial Cut}

The target fiducial cut removes events with vertices reconstructed outside of the target cell volume, such as those events originating from the target cryostat. The target cell volume is from $-110 \mathrm{~cm}$ to $-70 \mathrm{~cm}$ in the $z$ axis with a $2 \mathrm{~cm}$ radius. The $z$ axis target fiducial cuts as well as event vertices for the missing antiproton 


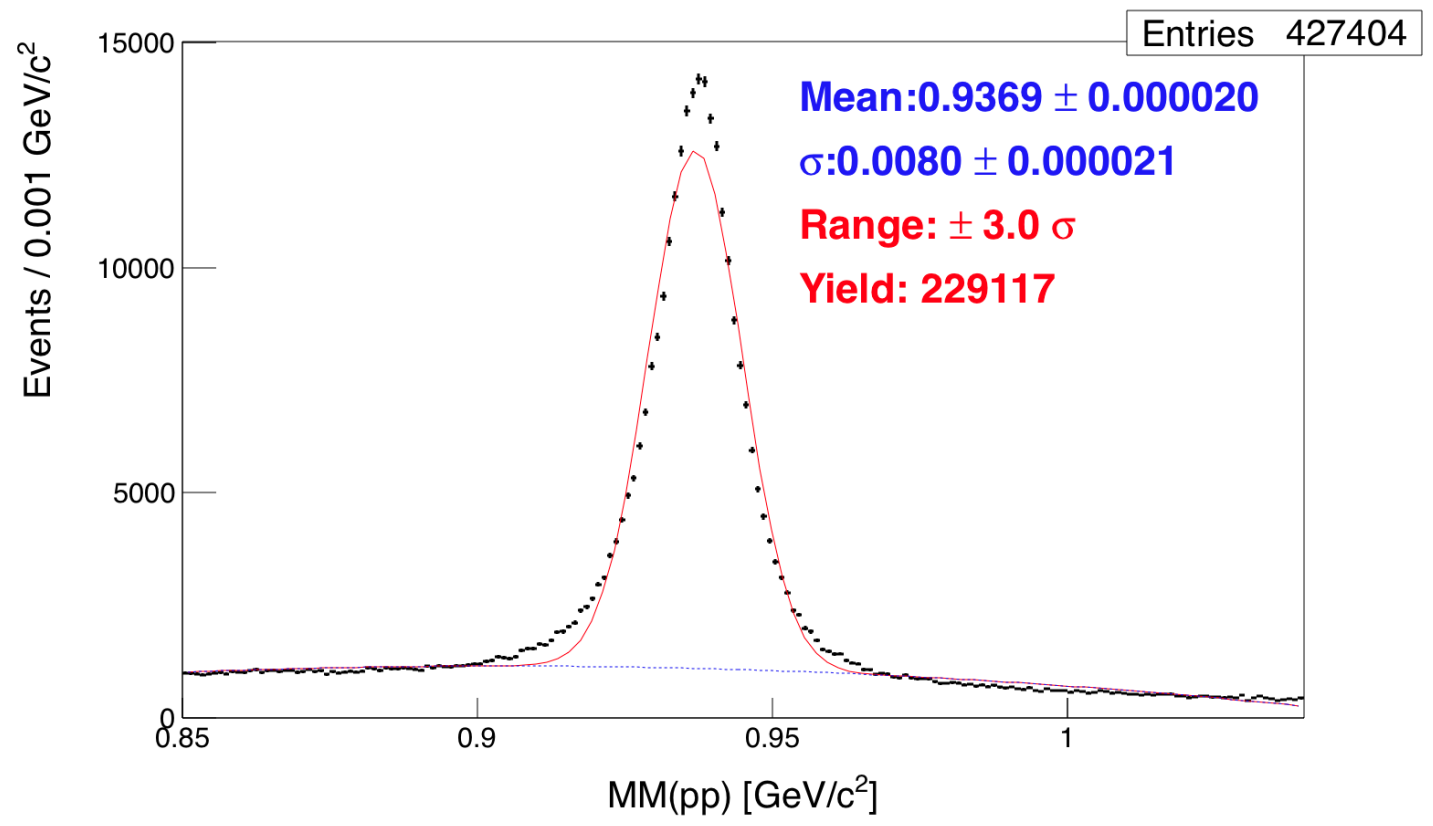

Figure 3.1: Missing mass off of $p p$ showing a missing $\bar{p}$, for the reaction $\gamma p \rightarrow$ $p p(\bar{p})$ which includes events where the $\bar{p}$ was also reconstructed.

topology are shown in Figure 3.3. In addition to a target length cut, there is also a target radius cut at $2 \mathrm{~cm}$ (Fig. 3.4).

\subsubsection{Detector Fiducial Cut}

In addition to the target vertex cut, we must also remove events where the momentum vector points to a non-active detector region, such as where the torus coils are located. The reason for removing these areas are a result of the inability to accurately determine detector efficiency in these regions. Figure 3.5 shows the reconstructed $\theta$ vs $\phi$ angle of the two protons in the missing antiproton topology. The detector fiducial cuts were applied in this figure and were determined by Jason Bono [23]. 


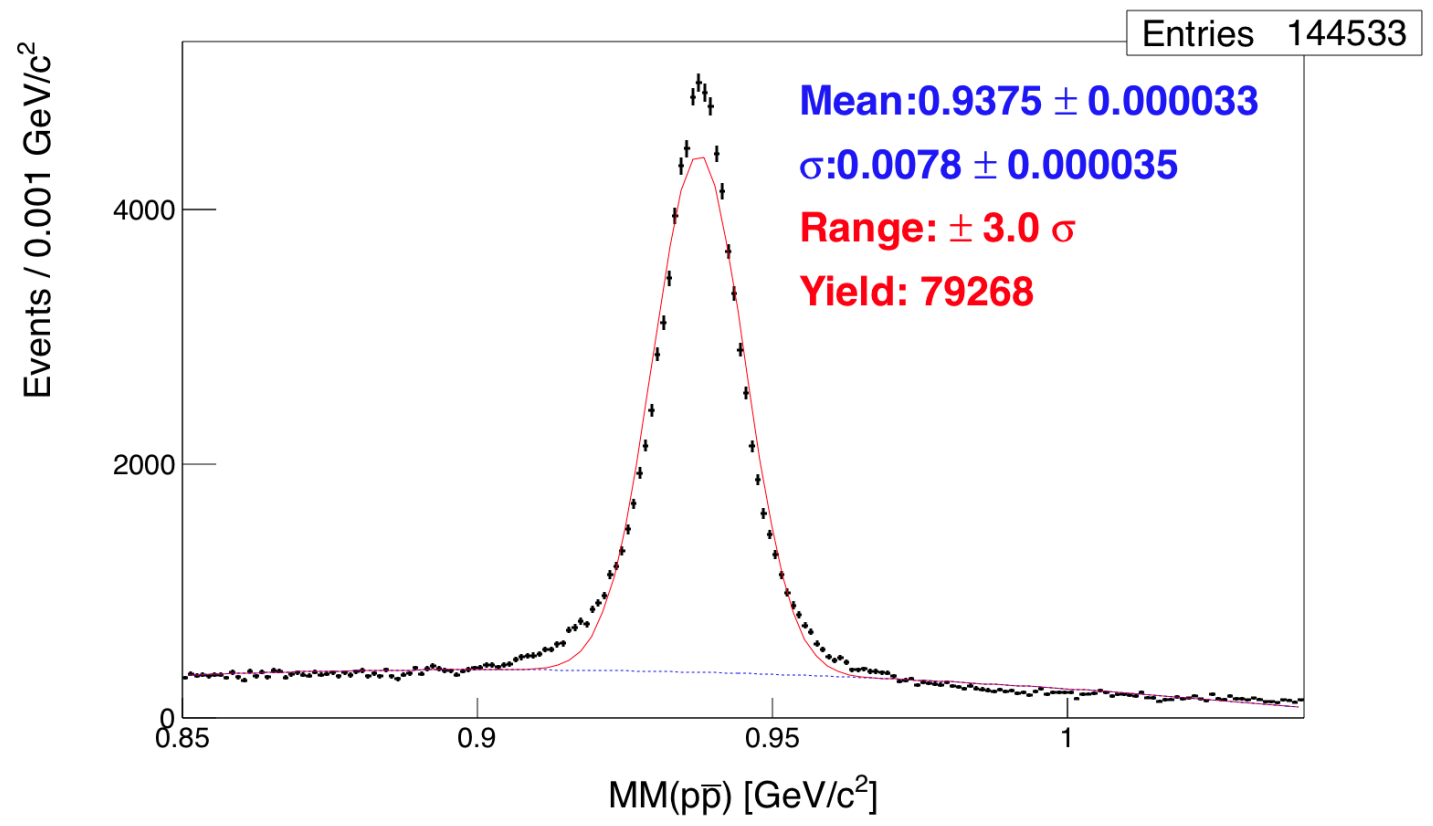

Figure 3.2: Missing mass off of $p \bar{p}$ showing a missing $\mathrm{p}$ (right), for the reaction $\gamma p \rightarrow p \bar{p}(p)$.

\subsubsection{Time-of-Flight Knock Outs}

In addition to removing events that fall outside of detector fiducial region and where the event vertex is outside of the target region, we must also remove areas with dead regions of the detector [23]. We handle dead or underperforming time-of-flight scintillating paddles by removing them. The time-of-flight study and cut was provided to the g12 group by Rafael Badui.

\subsection{Corrections}

All CLAS experiments need corrections applied to the reconstructed tracks before analysis. Energy Loss to account for energy lost in material prior to tracking, Momentum corrections to account for deviations from the ideal magnetic field of the torus, and beam energy corrections for photon experiments. 


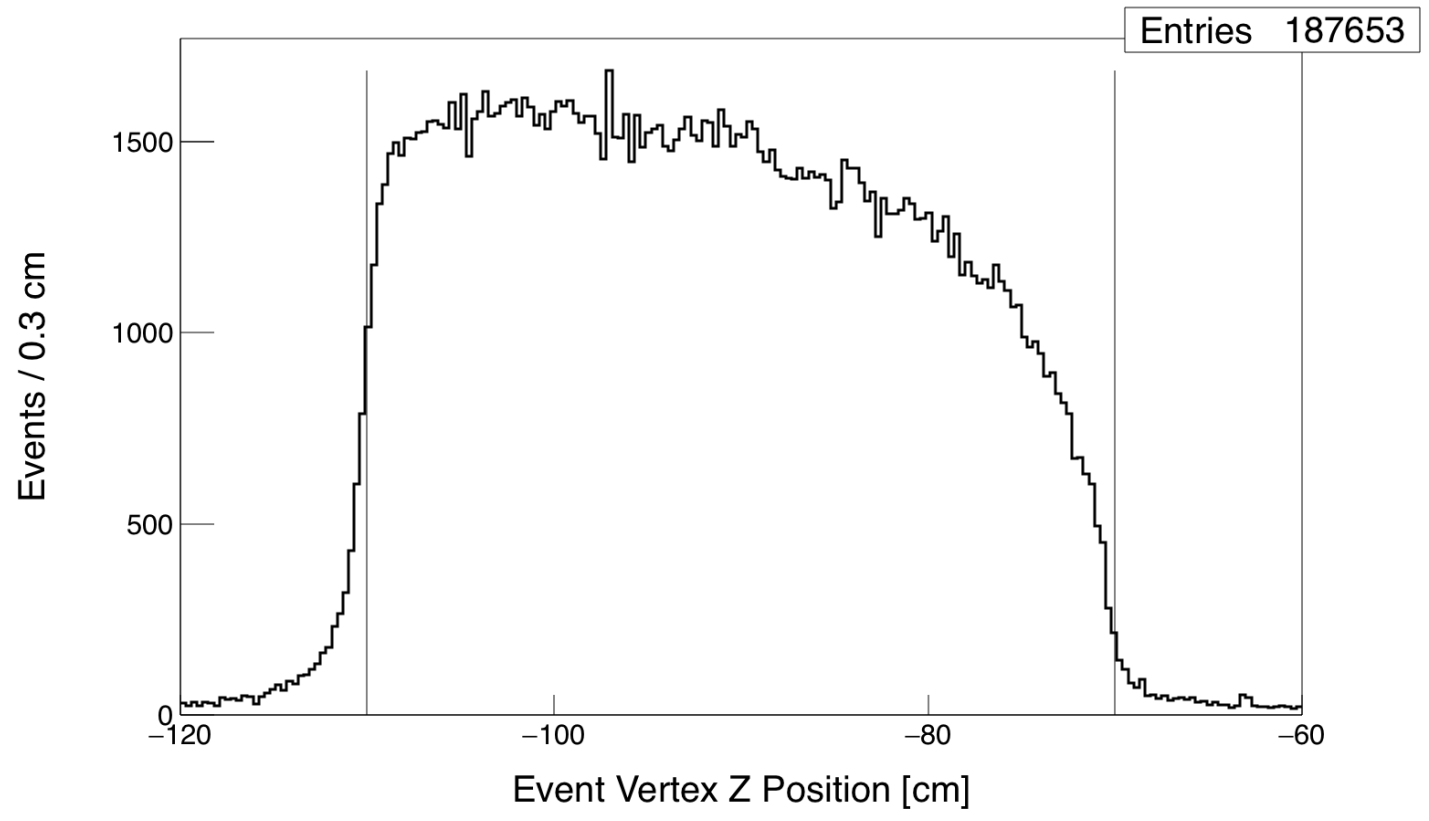

Figure 3.3: The event $z$ vertex distribution after all corrections and antiproton selection. Cuts were placed at the physical limits of the target from $-110 \mathrm{~cm}$ to $-70 \mathrm{~cm}$.

\subsubsection{Energy Loss}

The energy loss package is a standard CLAS package used to account for energy lost in media prior to the drift chambers [24]. The drift chambers provide a reconstructed momentum after the particle enters the drift chambers and this correction provides the momentum at the event vertex. The correction includes the energy lost in the target, target cell wall, target cryostat, start counter, etc.

\subsubsection{Momentum Correction}

The magnetic field of CLAS is known but with some imperfections since it was calculated and not mapped with magnetic field probes. That means that any slight deviation in coil position would lead to a deviation between the actual and calculated magnetic field. The difference between the calculated and actual 


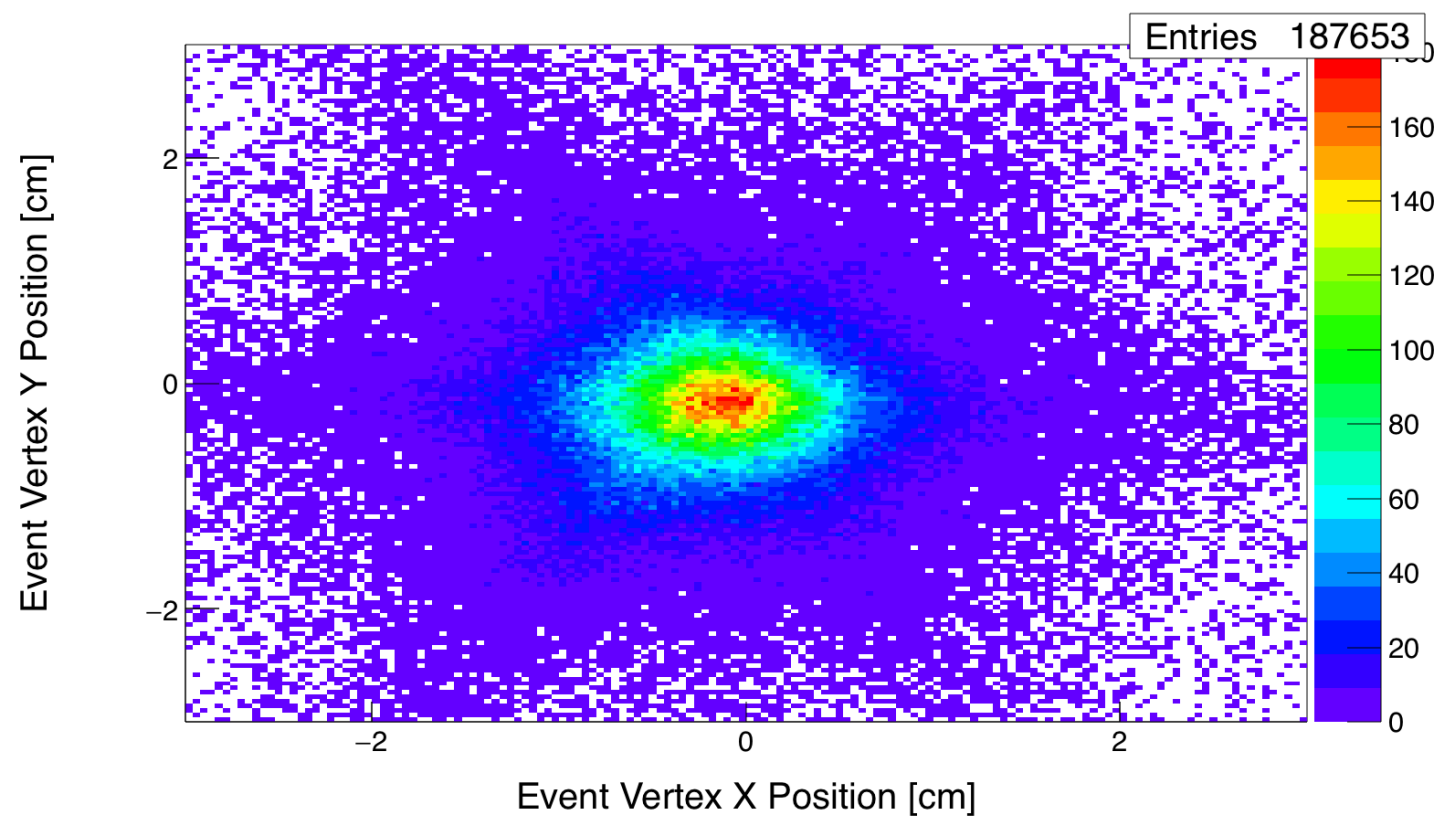

Figure 3.4: The event $x$ vertex vs $y$ vertex distribution after all corrections and antiproton selection. Cuts were placed at the physical limits of the target which has a radius of $2 \mathrm{~cm}$.

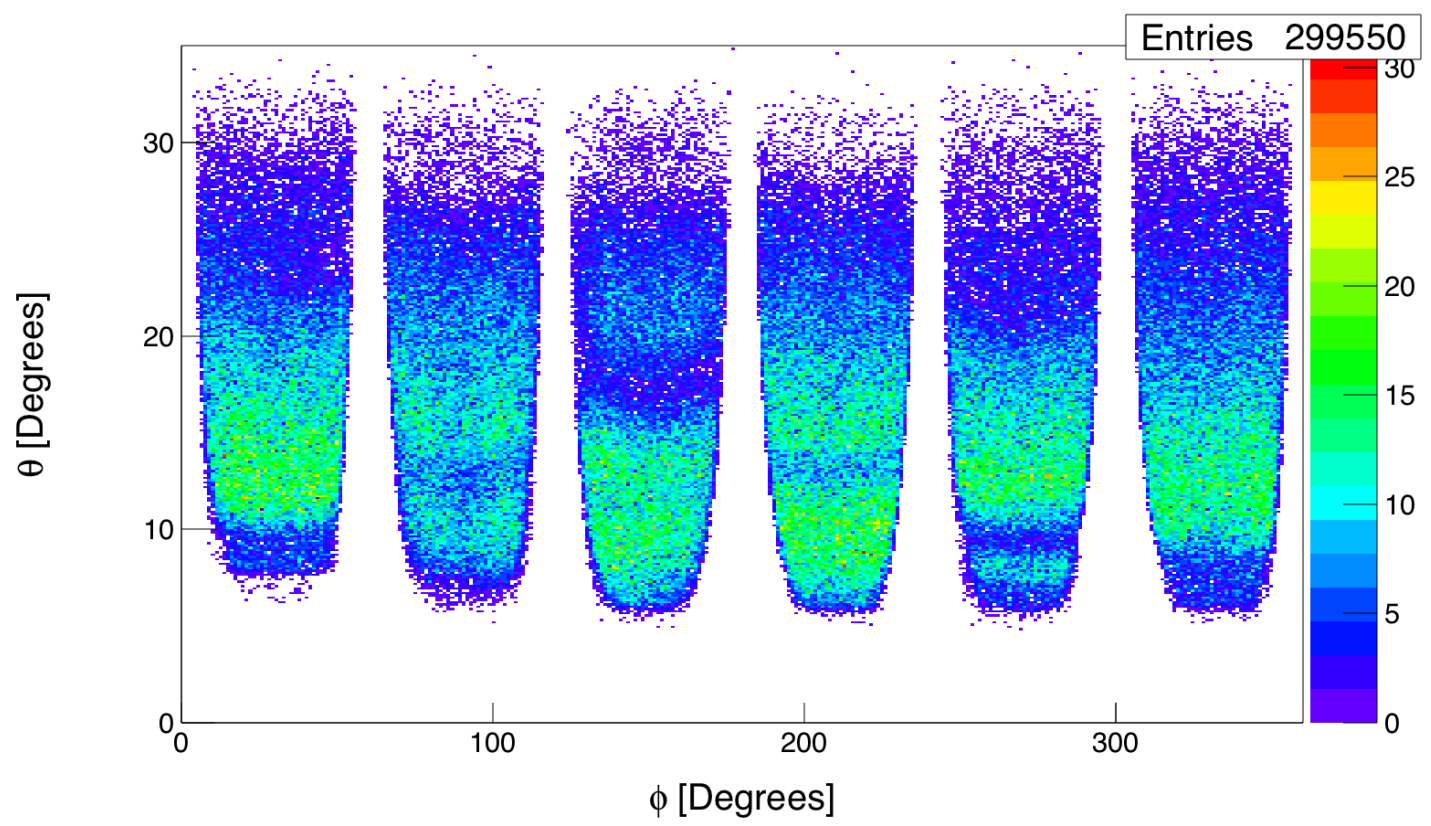

Figure 3.5: The angular distribution of the two proton tracks showing the holes in the detector; this is after applying fiducial cuts with the final event selection. 
magnetic field necessitates a correction to the momentum of the particles. The correction for this experiment was done by Johann Goetz and was done primarily using the reaction $\gamma p \rightarrow p \pi^{+} \pi$ - to derive a $\phi$ dependent correction factor [23]. The magnitude of this correction factor is typically small, on the order of a few $\mathrm{MeV}$. The momentum correction affect can be seen in Figures 3.6 (before) and 3.7 (after) for the fast proton. In this case the $\phi$-dependent momentum correction does not account for such high momentum protons, a further correction to the momenta after kinematic fitting desribed in Section 3.4 .

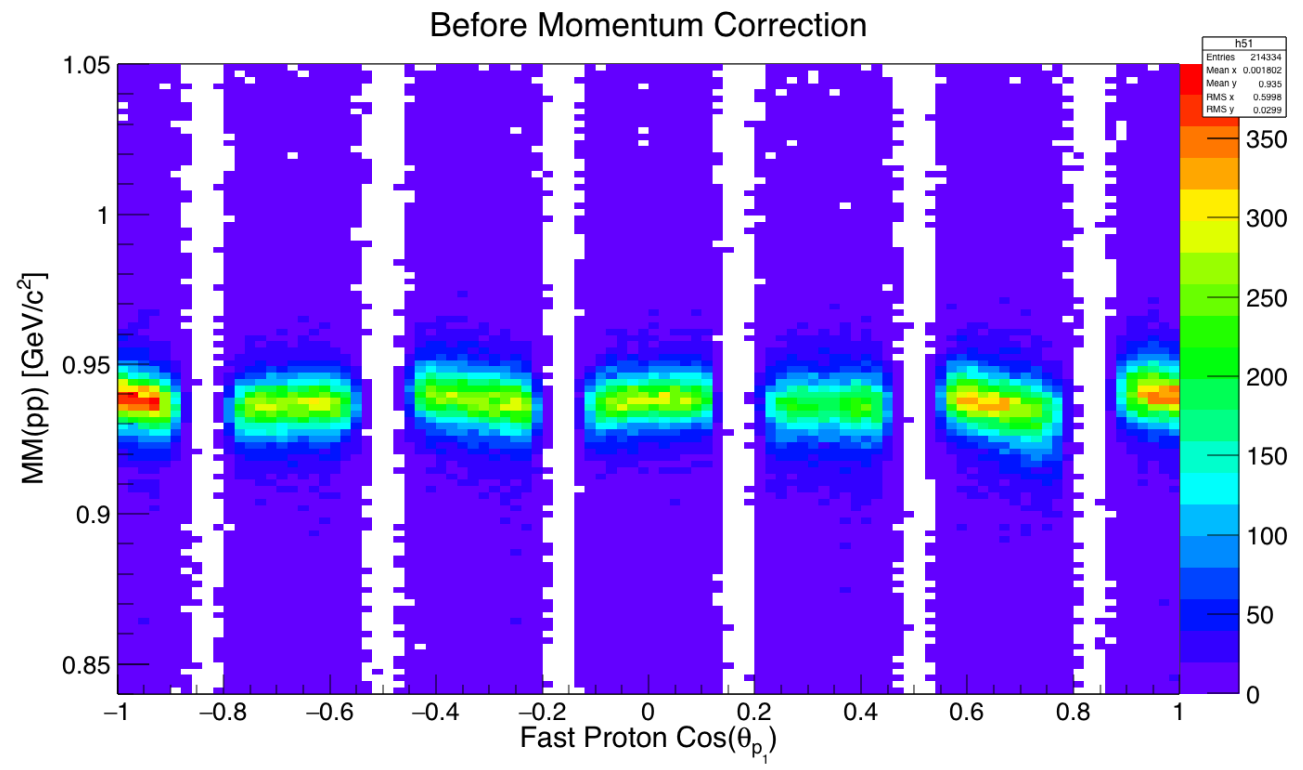

Figure 3.6: The missing mass as a function of the $\cos (\theta)$ of the fast proton showing an angular dependence of the momentum reconstructed by the drift chambers resulting in a skewness to the missing mass distribution.

\subsubsection{Beam Energy Correction}

During the analysis stage, particle masses appeared to be systematically low and were run dependent, showing steps in the run range. This discrepancy in particle masses was investigated using the reaction $\gamma p \rightarrow p \pi^{+} \pi^{-}$. The missing 
mass off of the two pions should give the proton mass, however it is clearly shown that this was not the case in Figure 3.8. In order to rule out an issue with reconstructed track momentum of the two pions, the invariant mass of a $K^{0} \rightarrow \pi^{+} \pi^{-}$was checked and it does not change meaningfully for the different run ranges. The study using the reaction $K^{0} \rightarrow \pi^{+} \pi^{-}$revealed that the issue was indeed with the tagger and the photon energy correction was calculated and verified with the reaction $\gamma p \rightarrow p \pi^{+} \pi^{-} n$. The effect of these corrections on this topology is shown in Figure 3.9. These corrections were applied run-by-run and were derived by Mike Kunkel [23].

\subsection{Reaction Reconstruction and Signal Extraction}

\subsubsection{Missing mass technique}

In order to take advantage of knowing the initial photon energy as well as knowing that the target is at rest we utilize a technique called missing mass. The missing mass technique allows the determination of the momentum of a missing final-state particle by using conservation of energy and momentum. The missing four-momenta is defined as

$$
P_{\text {miss }}=P_{\text {beam }}+P_{\text {target }}-P_{1}-P_{2}
$$

where $P_{\text {miss }}$ can later be identified as the momentum vector of a proton or antiproton. $P_{1}$ and $P_{2}$ are the remaining particles, either two protons or a proton and an antiproton. The importance of using this technique is that it allows you to recover events where one track (or more than one track) was not reconstructed as shown in Figures and. In the case of the missing antiproton topology, there are approximately 3 times more events when analyzed using the missing mass technique instead of requiring all of the final state particles be reconstructed. 


\subsection{Kinematic Fitting}

Kinematic fitting is a least-squares fit where individual components of the reconstructed four-momenta and the photon energy are the fit parameters and are free to vary. The adjustment to the reconstructed momentum is made to satisfy conservation of energy and momentum and utilizes the track covariance matrix from the reconstruction to determine the $\chi^{2}$ of the fit. The easily understood fit parameters such as $P_{p_{1}}, P_{p_{2}}$, and $P_{\gamma}$ correspond to the magnitude of the momentum vector of tracks and the beam energy. The fit parameters for each track, $p$, $\phi$, and $\lambda$, are in a local tracking frame. The local tracking frame is defined where the $\mathrm{x}$ axis is along the beam axis and the $z$ axis is in the direction of the Poynting vector of the magnetic field at the location of the track. The $y$ axis is defined as orthogonal to the $x$ and $z$ axis passing through the sector. The $\lambda$ parameter corresponds to the angle between the track and the $x-y$ plane and the $\phi$ parameter corresponds to the angle between the track and the beam line.

The kinematic fitter was tuned on the $p \pi^{+} \pi^{-}$reaction with resolution parameters optimized by Daniel Lersch [25]. This specific implementation of the fitter was written by Dustin Keller for the CLAS collaboration [26].

\subsubsection{Kinematic fitting results}

The kinematic fit results show a non-zero pull distribution for several parameters but a good Gaussian width of approximately 1.o. Ineffective momentum corrections could be to blame for the non-zero pull distributions. The momentum distribution for the protons in this reaction is higher than the topologies that were used to derive the corrections, which would cause the corrections to under correct the data. The non-zero kinematic fit parameter for the proton mo- 


\begin{tabular}{c|c|c|c|c} 
Parameter & Data Mean & MC Mean & Data $\sigma$ & MC $\sigma$ \\
\hline$p_{p_{1}}$ & -0.16 & 0.03 & 0.96 & 0.98 \\
$p_{p_{2}}$ & -0.02 & -0.01 & 0.94 & 0.97 \\
$\lambda_{p_{1}}$ & -0.18 & 0.04 & 0.96 & 0.99 \\
$\lambda_{p_{2}}$ & -0.19 & 0.03 & 0.95 & 0.99 \\
$\phi_{p_{1}}$ & -0.04 & 0.03 & 0.96 & 0.98 \\
$\phi_{p_{2}}$ & -0.03 & -0.01 & 0.95 & 0.98 \\
$E_{\gamma}$ & 0.19 & -0.04 & 0.96 & 0.99 \\
\hline
\end{tabular}

Table 3.3: Table of Gaussian widths and means from a fit to the kinematic fit parameter pull distributions from Figures 3.10 and 3.11 . The experimental data shows a shift in the mean which could be due to the momentum corrections not being suited for this reaction.

mentum $\left(p_{1}\right)$ and the $\lambda$ parameters could be related to the $\phi$ asymmetry shown in Figure 3.10 and Table 3.3

When running the kinematic fitter with the same resolution scaling parameters and parameter files we see good momentum distributions and pull distributions. All centered near o and with a Gaussian sigma of 1.0, seen in Fig. 3.11. Monte Carlo does not suffer from such defects that require corrections, so one could propose that the shifted means in the pull distributions in data come from defective corrections, or from corrections that do not cover the same momentum range. One should look at the shifted means in the pull distribution and see a correction that is being applied as a final tune to the data for this particular topology and not that there is something wrong with the fitter.

\subsubsection{Event Selection with Kinematic Fitting}

When using kinematic fitting for event selection there is some over counting due to the inclusion of background events into the signal. Figure 3.12 shows the results of using kinematic fitting for event selection. 


\subsection{General Features of the data}

General features of the data are shown from Fig. 3.13 to Fig. 3.18 prior to detector efficiency corrections. The minimum photon energy for which $p p \bar{p}$ events are detected is $3.95 \mathrm{GeV}$, which is above the reaction threshold of 3.75 $\mathrm{GeV}$ in which the particles have zero momentum in the CM frame Fig.3.13. The maximum photon energy in which this reaction is seen is $5.45 \mathrm{GeV}$, which is limited by the energy of the photons.

The momentum distribution shown in Fig. 3.14 is of the final-state particles and shows their momentum distribution ranging from $500 \mathrm{MeV}$ to $3.5 \mathrm{GeV}$. The lower limit of the momentum distribution is limited by the acceptance of CLAS for low momentum particles, which are bent into the non-active detector regions more readily than the faster particles. The upper limit is determined by the maximum beam photon energy. The antiproton has a slightly lower momentum than the fast proton but significantly more than the slow proton.

The detector inefficiencies can be clearly seen in the $\theta$ vs $\phi$ angular distribution of the two detected protons shown in Figure 3.5. At lower angles in $\theta$ the detector has much lower $\phi$ coverage than the higher angles due to the configuration of the torus coils applying spacial constraints. At $\theta<7.5^{\circ}$ there is the hole that allows high energy recoil electrons to pass through the detector so that the detector is not saturated with those events that are not of interest.

\subsubsection{Mass distributions}

In prior experiments narrow resonances were reported in the $p \bar{p}$ mass spectrum for more than one experiment with one paper claiming to have comprehensive evidence for narrow resonances [9]. These resonances were reported as 
enhancements in the mass distribution of the $p \bar{p}$ pair at 2.02 and $2.2 \mathrm{GeV}$. The mass distributions prior to detector efficiency correction for the $p \bar{p}$ pair show no strong evidence for the 2.02 and $2.2 \mathrm{GeV}$ resonances in Figures 3.17 and 3.18 . In chapter 4 detailed cross section measurements $d \sigma / d M(p p)$ and $d \sigma / d M(p \bar{p})$ are shown where further lack of strong evidence for any narrow resonances can be seen. In the $p p$ invariant mass distribution there is also no evidence for a narrow resonance as well Fig. 3.16 .

\subsection{Monte Carlo Simulation}

Monte Carlo simulations are used to take into account the imperfections in the detector as well as the physical processes that can affect the ability for reconstruction to properly reconstruct a track. The goal is to measure a detector efficiency for each measurement in order to correct for any inefficiencies that are known. The detector efficiency, $\epsilon$, is defined as

$$
\epsilon=\frac{N_{\text {reconstructed }}}{N_{\text {generated }}}
$$

The CLAS detector simulations use the GEANT libraries, which take into account the physical location of the detectors and the physical processes that occur when a particle is traveling through different media. The program is GSIM and it simulates the detector response with perfect timing information and detector resolutions. The known inefficient detector channels, detector resolution, and timing resolution from calibrations are then applied to the simulated events in a program called GSIM Post Processor (GPP).

Although the features of the data are interesting, in order to understand more about the production mechanism cross sections must be calculated. An important part of calculating cross sections is accurately calculating detector 
efficiency using Monte Carlo simulations. Chapter 4 will discuss calculating cross sections and the importantance of Monte Carlo simulations. 


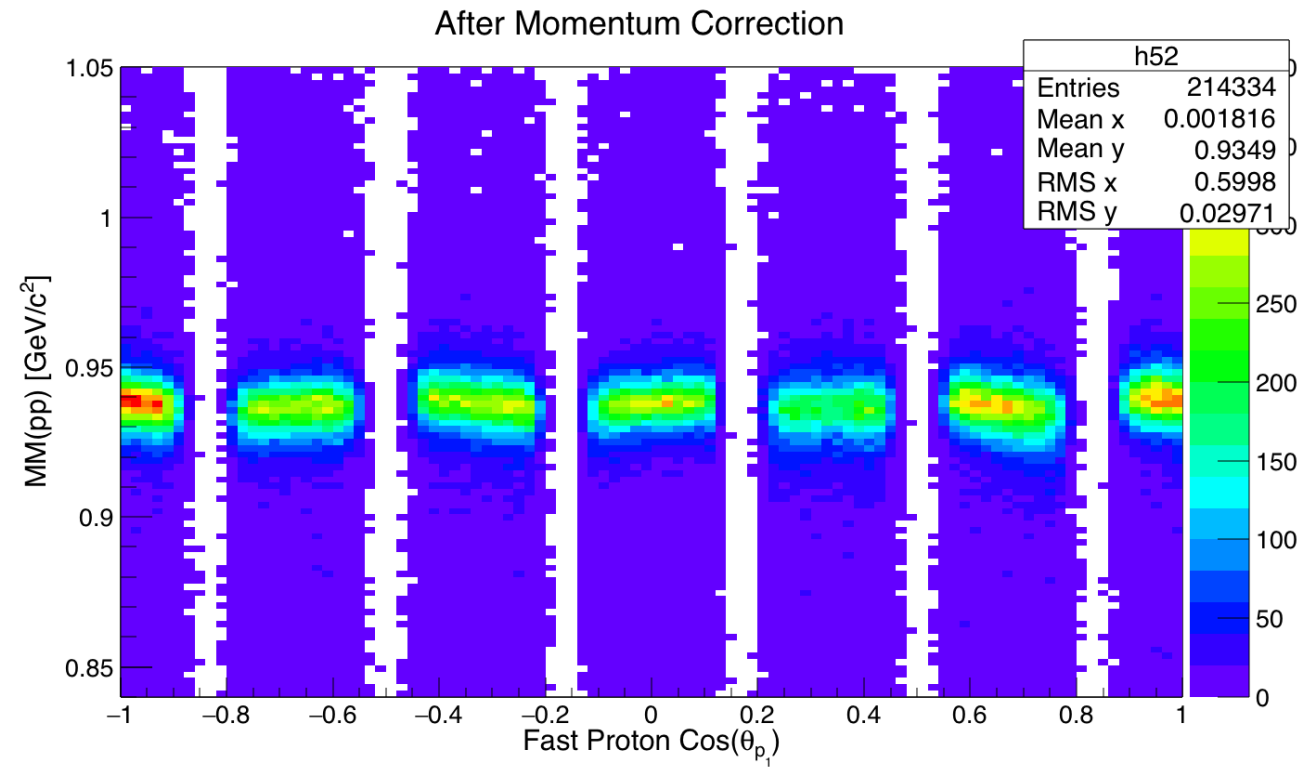

Figure 3.7: The missing mass as a function of the $\cos (\theta)$ of the fast proton after momentum corrections, showing a reduced skewness. However, due to the high momentum of the particles studied, the standard momentum corrections are insufficient and it is seen that there is little difference before and after applying the momentum correction. 


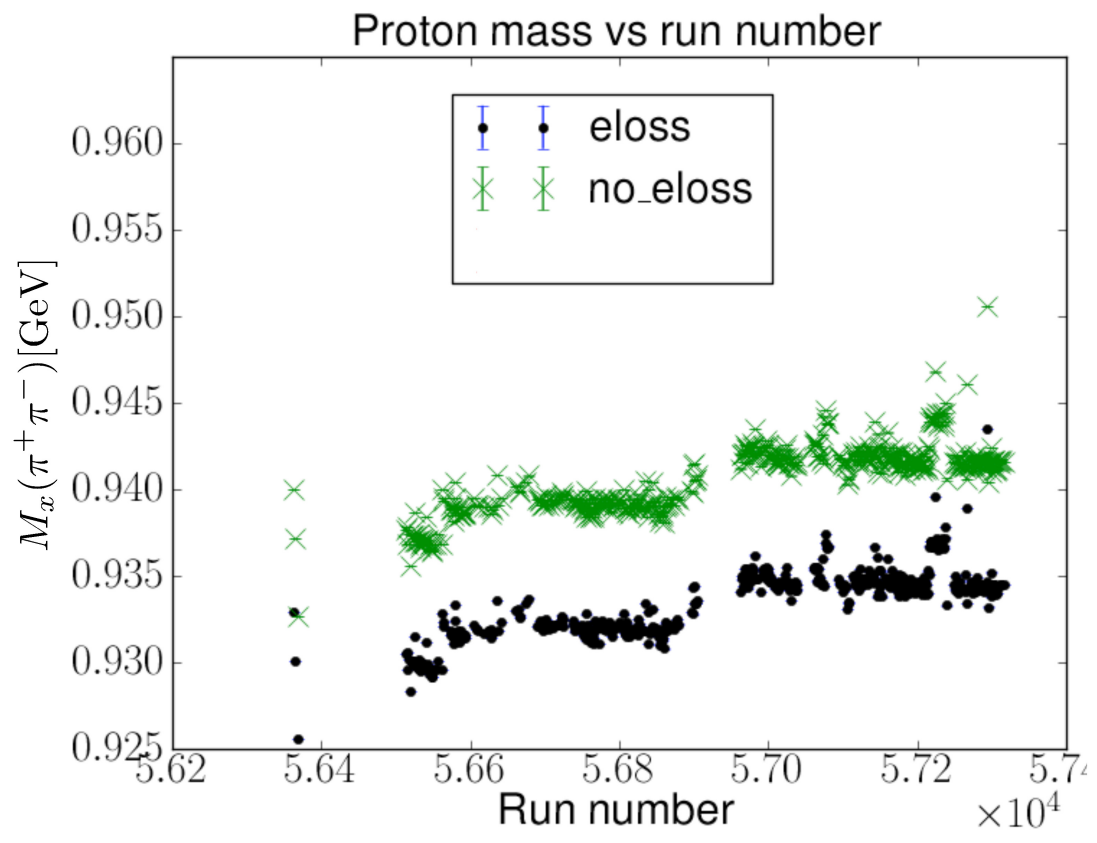

Figure 3.8: The missing proton mass distribution by run number in the exclusive reaction $\gamma p \rightarrow p \pi^{+} \pi^{-}$showing the effects of the energy loss package [23]. The missing proton mass is lower after the correction due to the pions having higher momenta after the correction. 


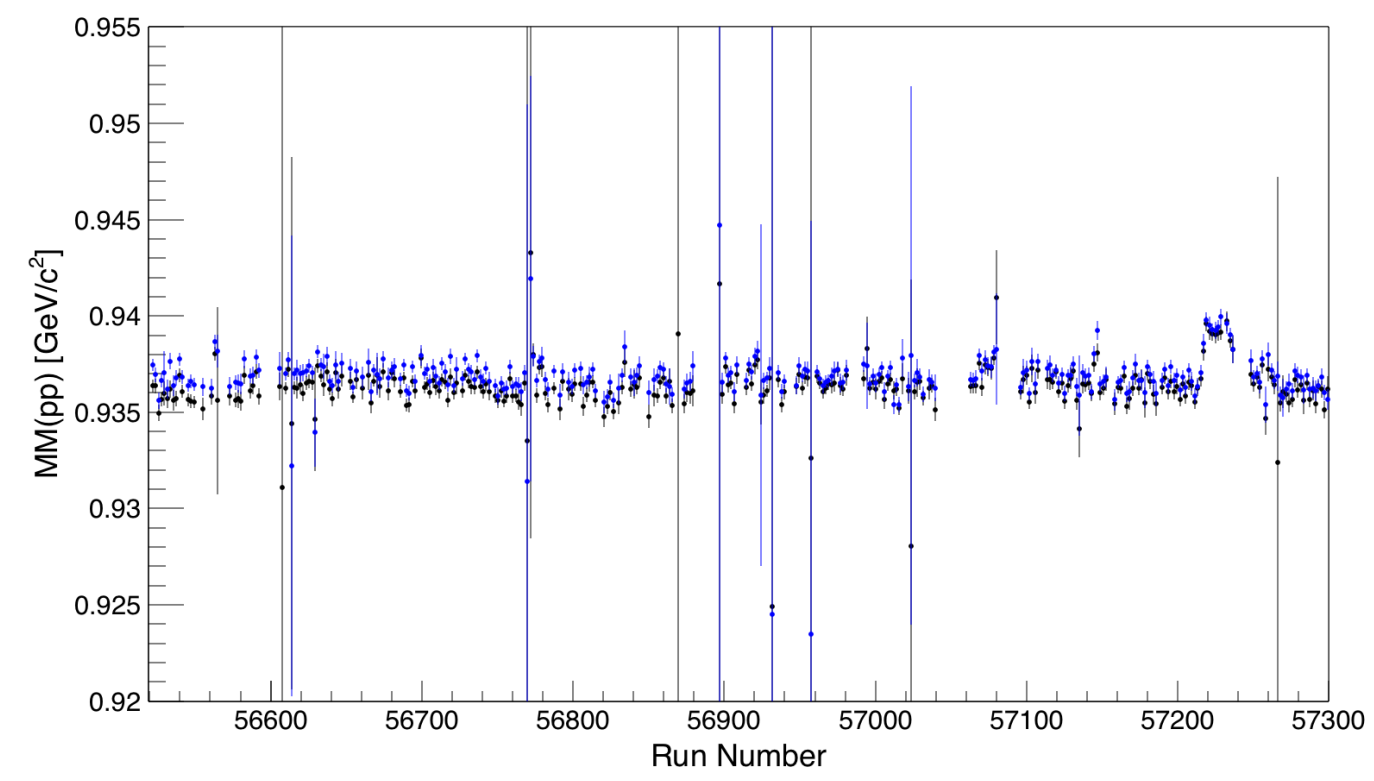

Figure 3.9: The mass dependence of the missing antiproton by run, before beam energy corrections shown in black and after beam energy corrections in blue.
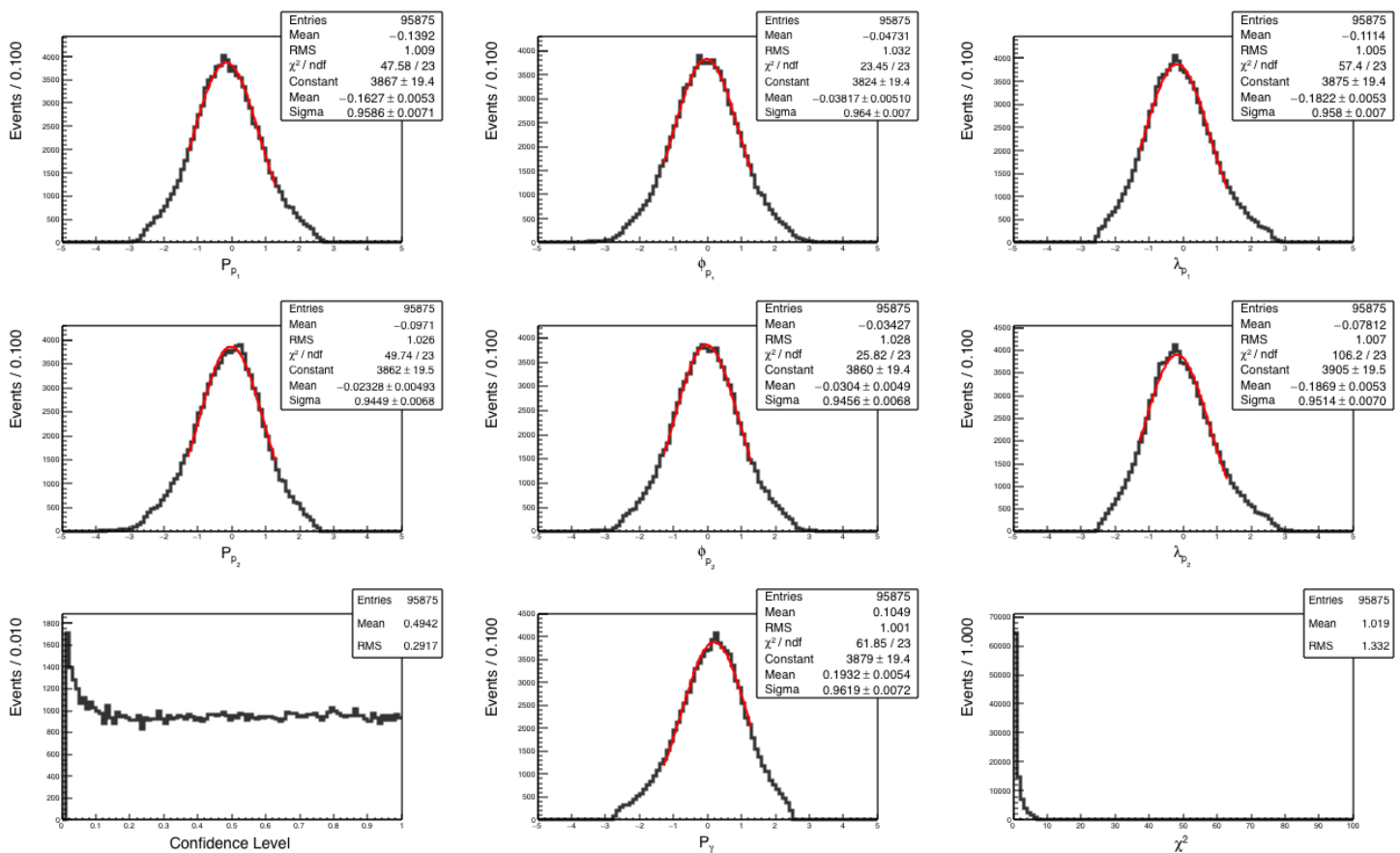

Figure 3.10: Kinematic fitting pull distributions and confidence level showing sigmas near 1 but with shifts in the mean of the distribution. Shown with a $1 \%$ cut on the pull probability distribution to reduce background. See Table 3.3 for Gaussian fit parameters. 

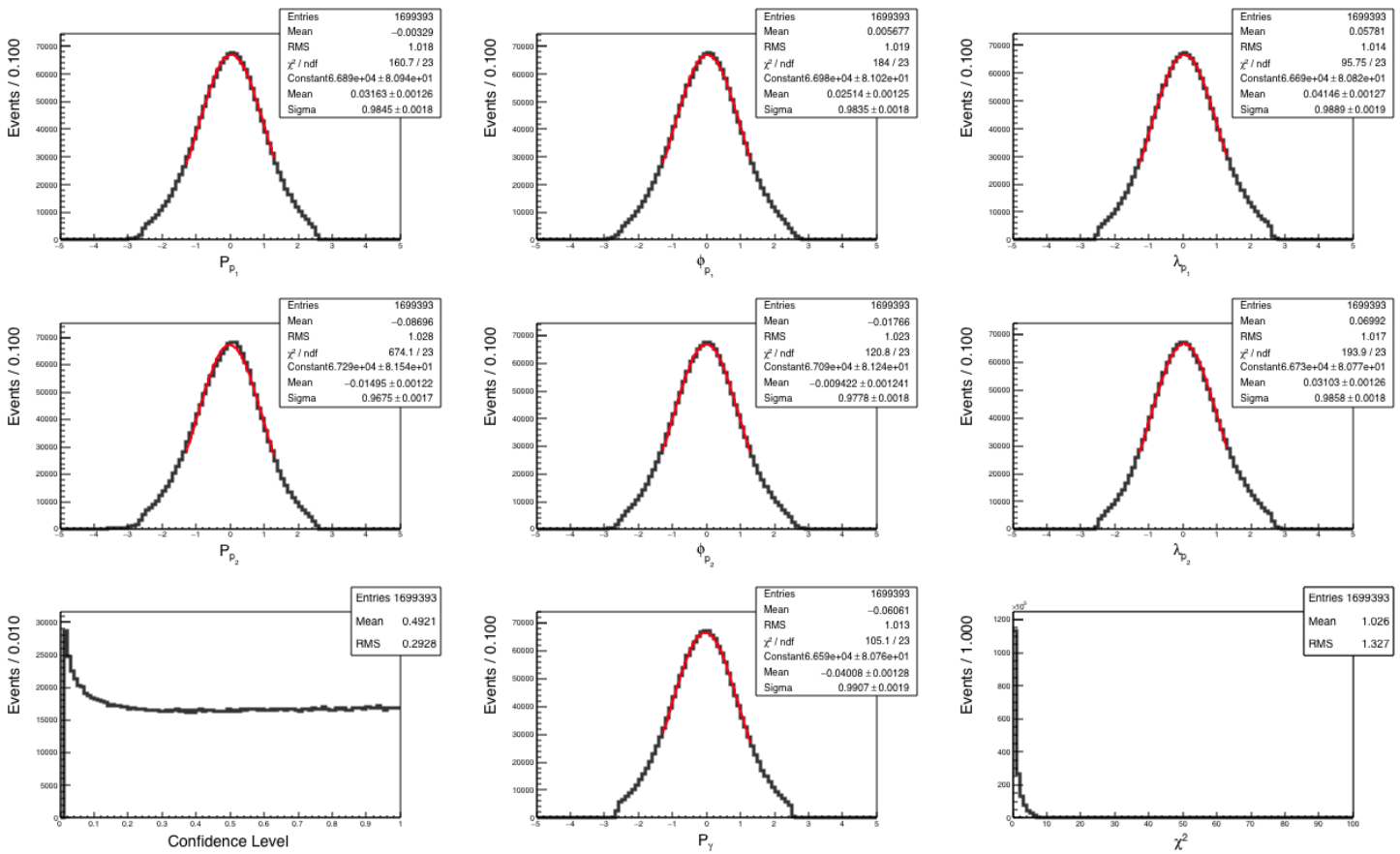

Figure 3.11: Kinematic fitting pull distributions and confidence level distribution for Monte Carlo events. With means near zero and Gaussian sigmas near 1.o it shows that the fitter is tuned well for this reaction. Shown with a $1 \%$ cut on the pull probability distribution to reduce background. See Table 3.3 for Gaussian fit parameters. 


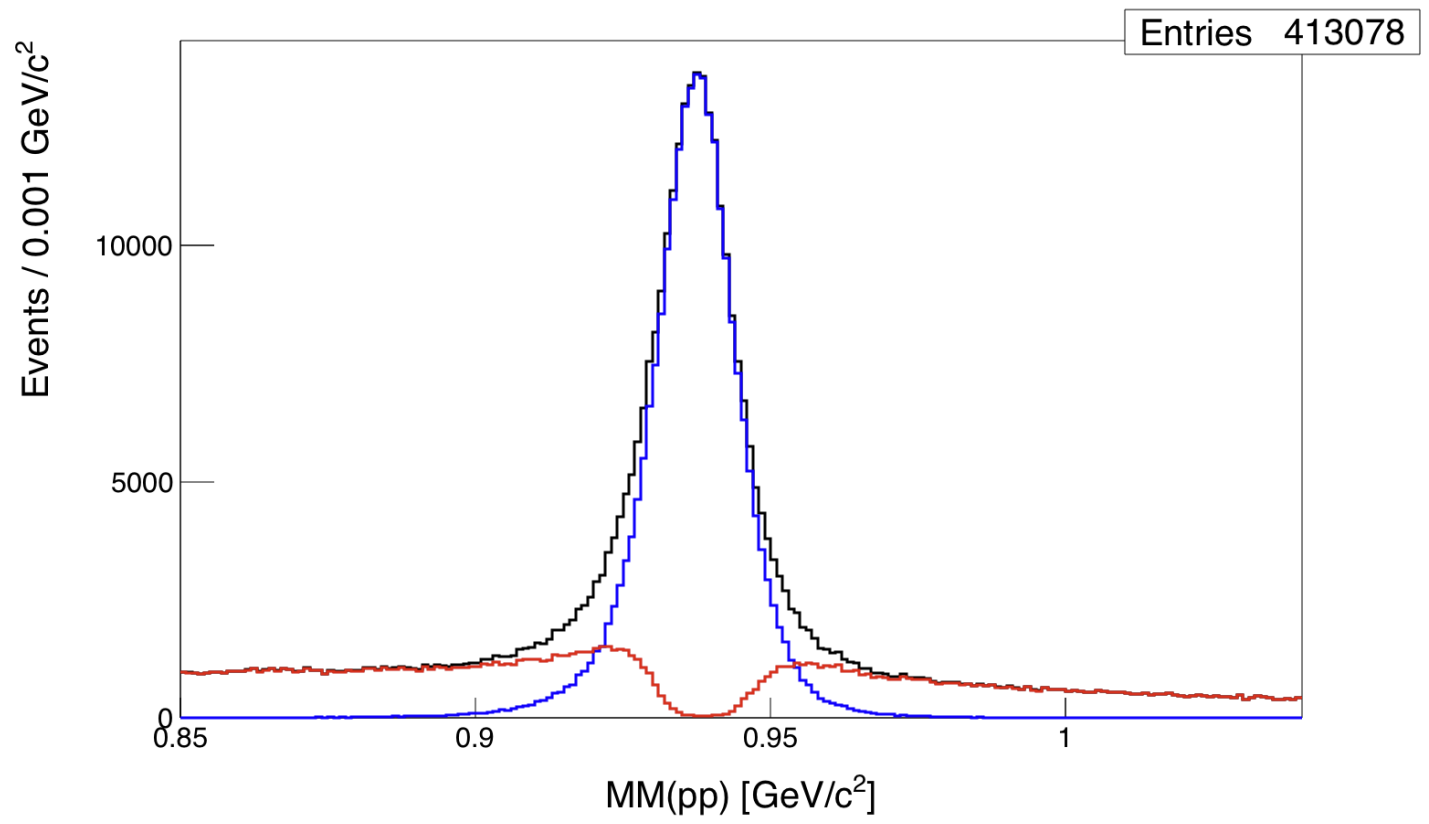

Figure 3.12: Missing mass off of $p p$ showing a missing $\bar{p}$, for the reaction $\gamma p \rightarrow p p(\bar{p})$. The red histogram shows the background distribution where the pull probability cut $<5 \%$ and the blue distribution shows the signal with a pull probability cut of $>5 \%$ 


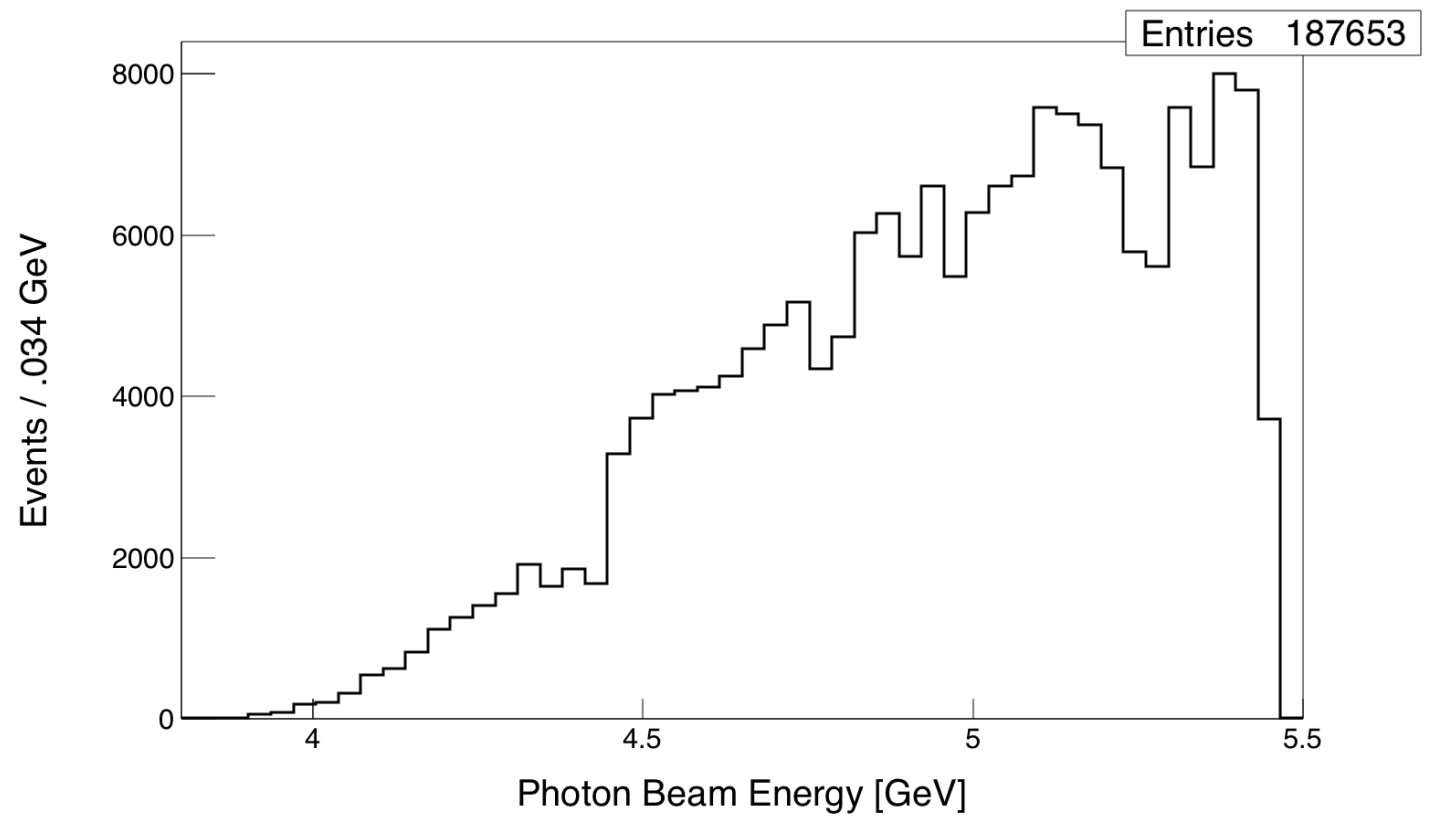

Figure 3.13: Beam energy distribution of events with all corrections and cuts applied. At $4.4 \mathrm{GeV}$ the trigger configuration changes and explains the sharp jump in the number events above $4.4 \mathrm{GeV}$. Additionally, the dips above $4.4 \mathrm{GeV}$ are due to inefficient paddles and are not seen in the total cross section. 


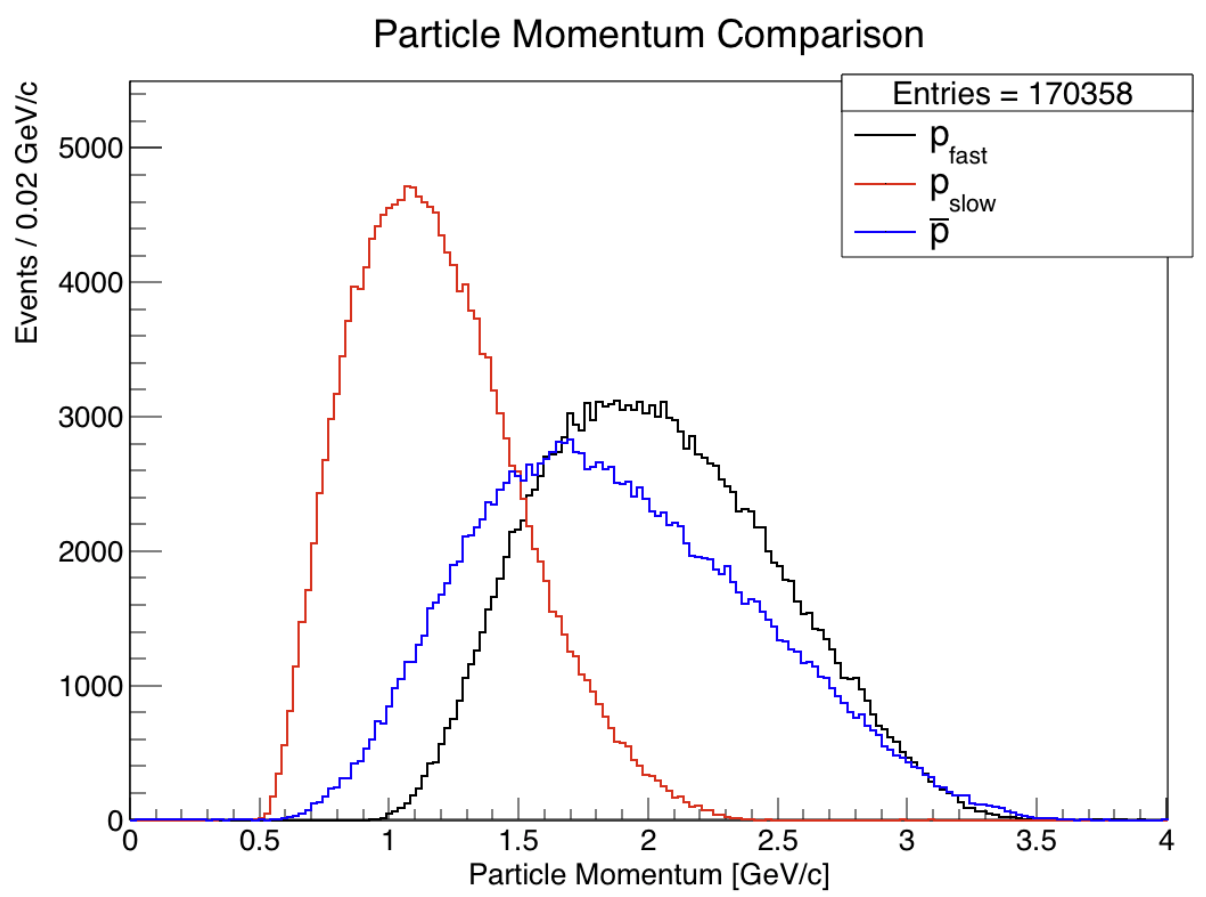

Figure 3.14: This figure shows the particle momentum distributions in comparison with one another. Detected particle momenta are all between $500 \mathrm{MeV}$ and $3 \mathrm{GeV}$

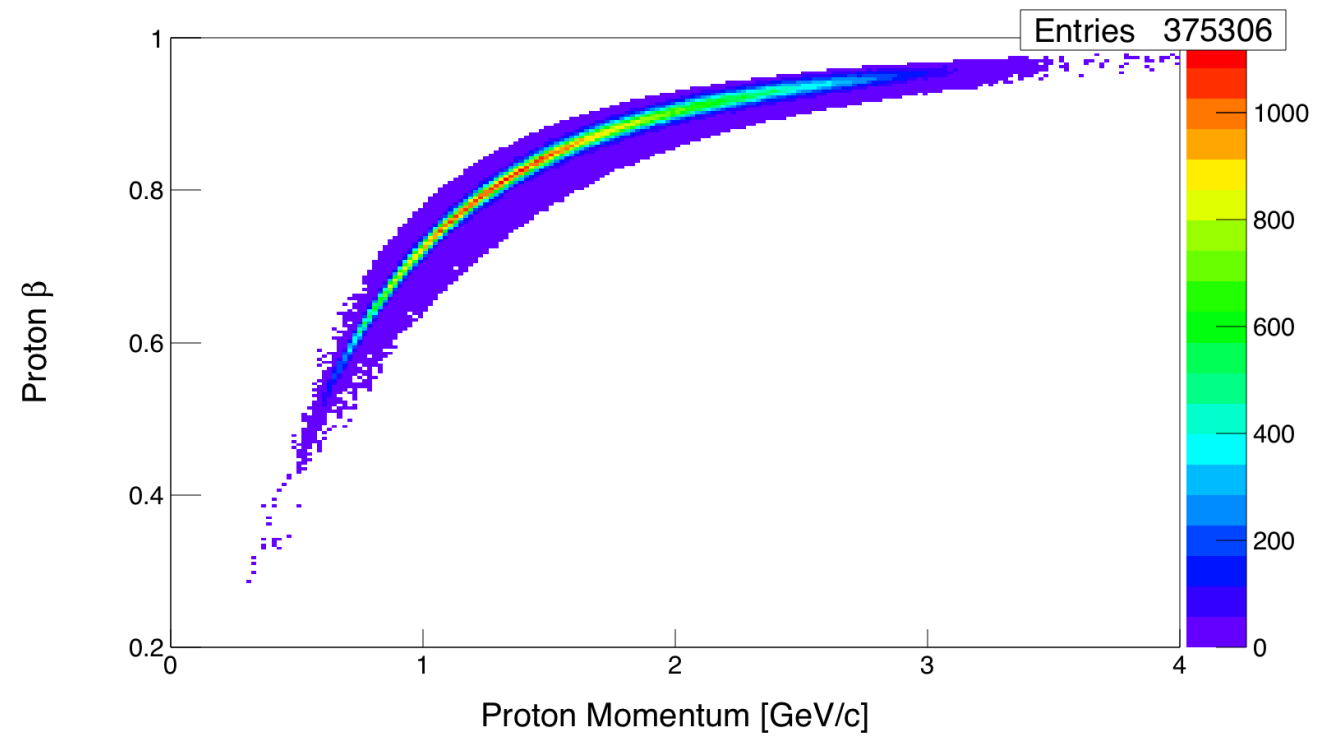

Figure 3.15: Momentum vs $\beta$ distribution showing no out of time bands that are typically evidence of misidentified protons after all analysis cuts. 


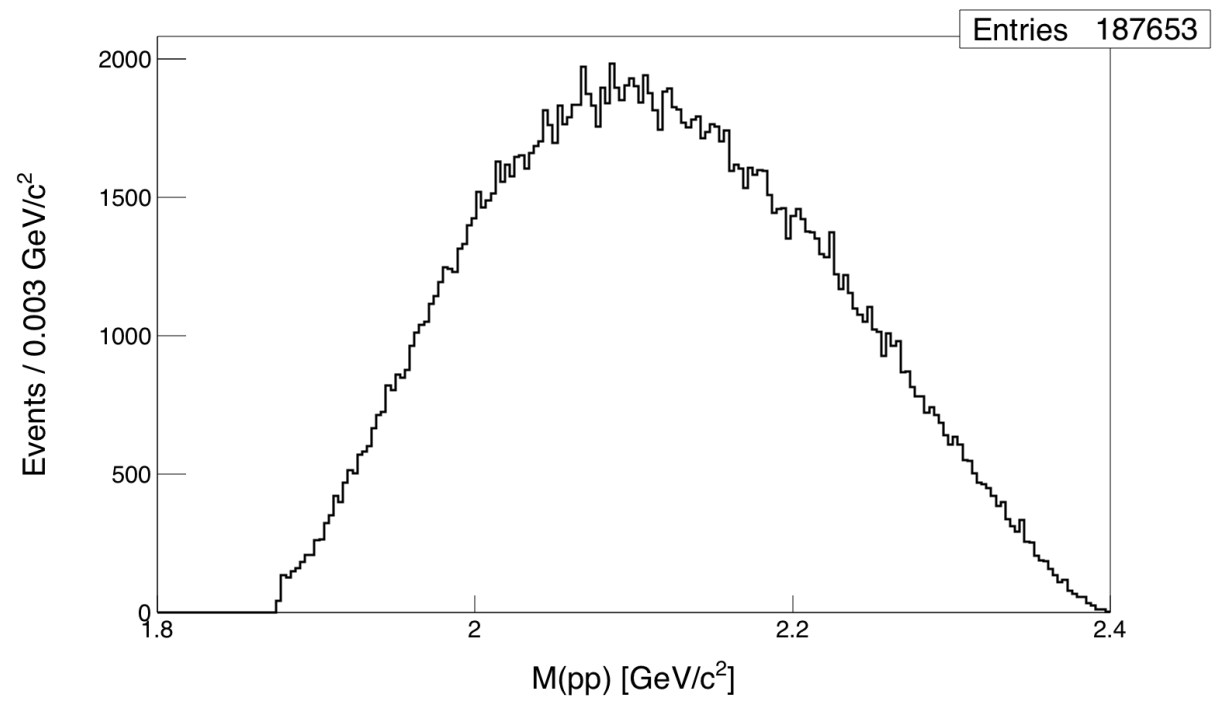

Figure 3.16: The invariant mass of the two protons for the final event selection.

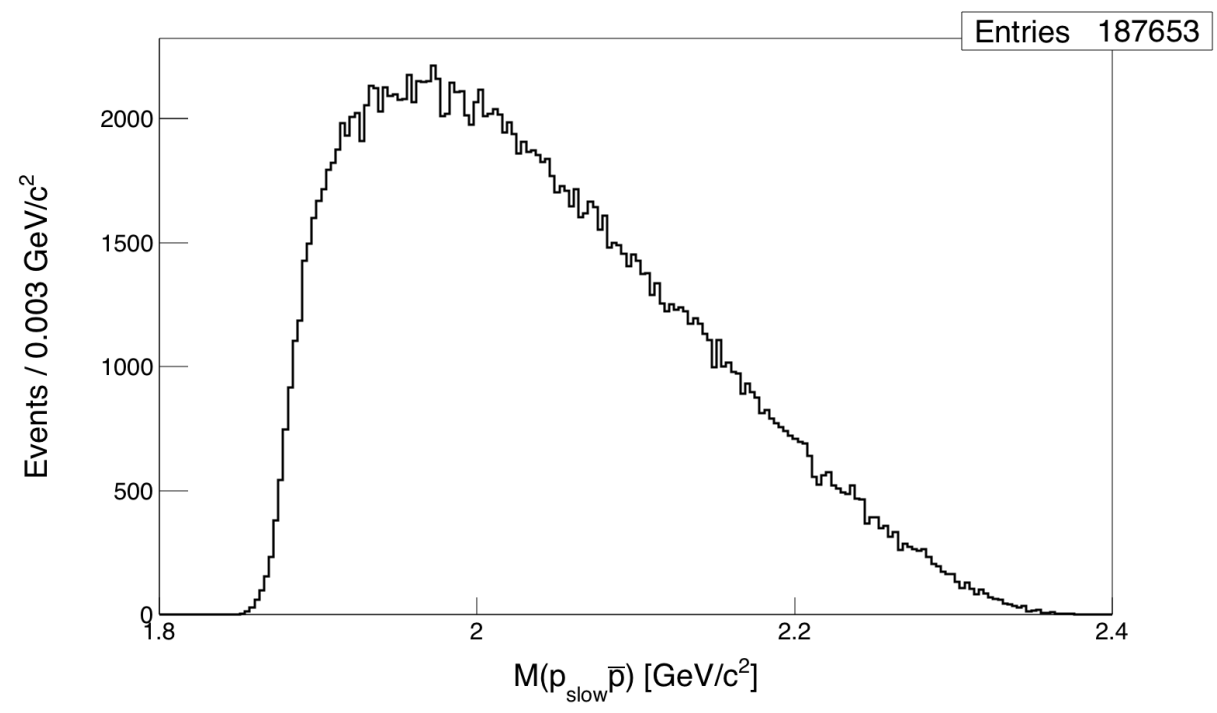

Figure 3.17: The invariant mass of the slow proton-antiproton pair for the final event selection. 


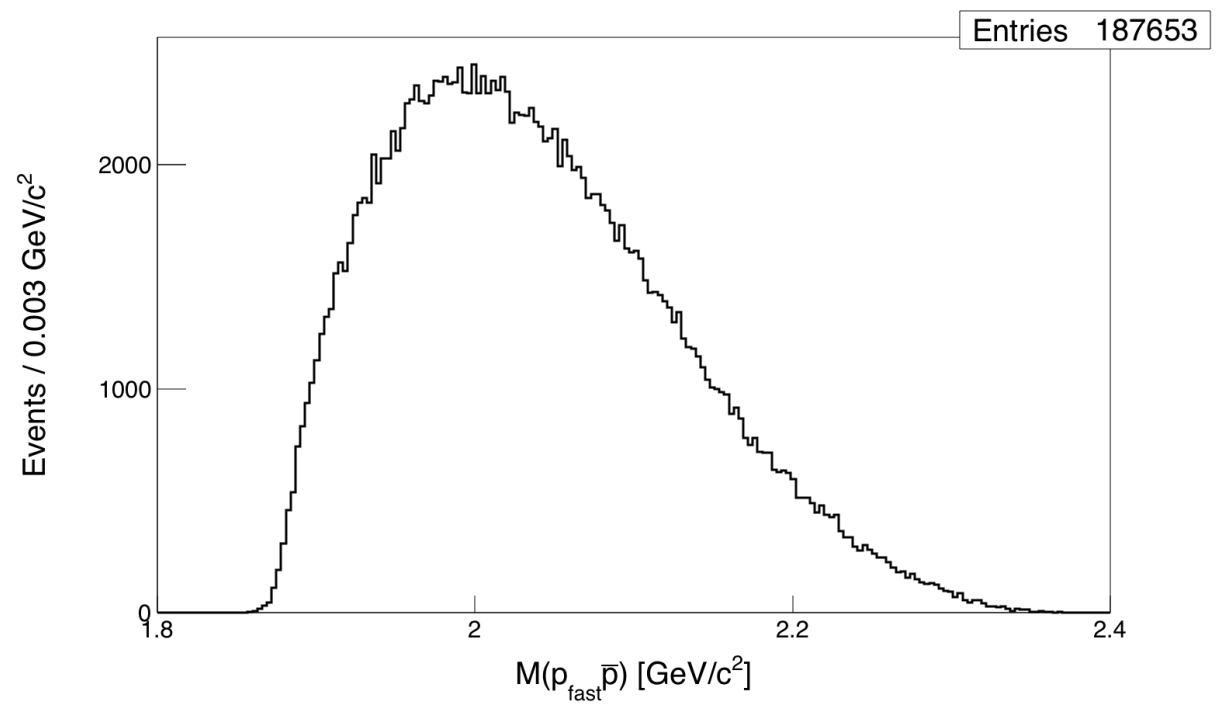

Figure 3.18: The invariant mass of the fast proton-antiproton pair for the final event selection.

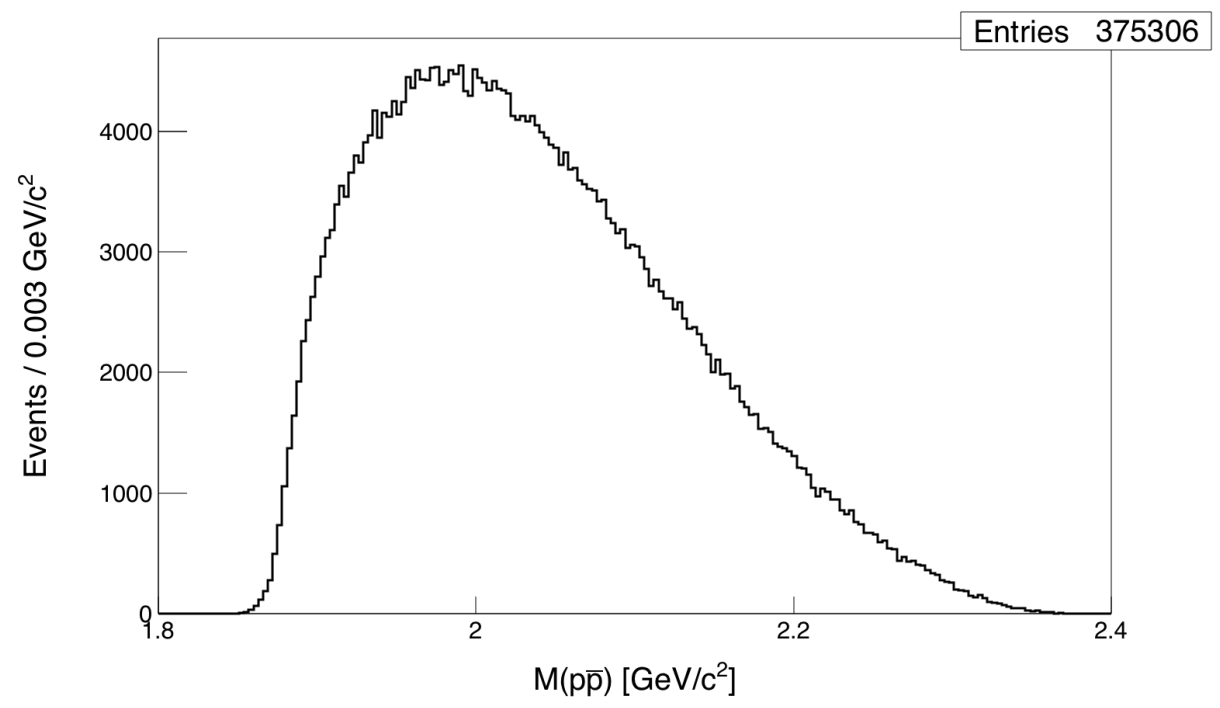

Figure 3.19: The invariant mass of all combinations of the proton-antiproton pairs for the final event selection. 


\section{CHAPTER 4}

\section{Cross section measurement of $\gamma p \rightarrow p p \bar{p}$}

\subsection{Overview}

In order to better understand the production mechanism of the reaction $\gamma p \rightarrow$ $p p \bar{p}$, the differential cross sections as well as the total cross section have been measured. This dataset provides orders of magnitude more statistics at 200,000 events compared with the previous published work which had 200 events. This level of statistics provides the opportunity to measure detailed differential cross sections $d \sigma / d \cos \left(\theta_{\bar{p}}\right), d \sigma / d M(p p), d \sigma / d M(p \bar{p})$ for the first time. With these measurements, theorists will be able to compare their predictions with the data and it will have the potential to produce new information about the photocouplings of the different production mechanisms.

\subsection{Definition}

The definition for a total cross section is defined as

$$
\sigma=\frac{N}{\epsilon \cdot L^{\prime}}
$$

where $N$ is the number of events, $\epsilon$ is the detector efficiency, and $L$ is the luminosity, which is defined as

$$
L=\frac{N_{\gamma} \cdot \rho_{\text {target }} \cdot L_{\text {target }} \cdot N_{A}}{w_{H}},
$$


where the target characteristics are defined in Table 4.1 and $N_{\gamma}$ is the photon flux incident upon target. The detector efficiency, $\epsilon$, is defined in Equation 3.2.

Table 4.1: Constants used for calculating the cross sections.

\begin{tabular}{c|c|c} 
Parameter & Value & Definition \\
\hline$\rho_{\text {target }}$ & $0.0711398 \mathrm{~g} / \mathrm{cm}^{3}$ & $\ell \mathrm{H}_{2}$ Target Density \\
$N_{A}$ & $6.022 \cdot 10^{23}$ & Avogadro's number \\
$L_{\text {target }}$ & $40 \mathrm{~cm}$ & Target length \\
$w_{H}$ & $1.00794 \mathrm{~g} / \mathrm{mol}$ & Atomic Weight for Hydrogen
\end{tabular}

\subsubsection{Yield Extraction}

The yield is calculated from the number of events passing a $5 \%$ confidence level cut. In order to correct for under or over counting, the missing mass distribution of the two protons is fit with a Gaussian + third order polynomial background to correct for the miscounting. Due to the signal distribution not taking a Gaussian shape, the background function is used instead in combination with an integral of the histogram to extract the yield. The resulting yield is taken from an integral with the range of $\pm 3 \sigma$ from the Gaussian mean of both the histogram and the background function. The yield is then

$$
\mathrm{Y}=N_{\text {histogram }}-N_{\text {background }}
$$

\subsection{Simulation and Acceptance Calculation}

In order to correct for the inefficiencies of the CLAS detector an accurate model must be used to generate events. If an incorrect model is used then the momentum and angular distributions of the particles will be different and if they are 
biased towards a portion of the detector with better or worse acceptance, then the cross section measurements will also be biased.

The first model that was tried was a t-channel diffractive model where the tunable parameters are the mass distribution of the $p \bar{p}$ pair and the t-slope. Many parameters were tried but a simple diffractive model proved to be insufficient. A more sophisticated technique was needed in order to match the simulated data with the experimental data. The technique that did work used the cross sections derived from an initial simulation and then generating events based on those initial cross sections. This process is repeated many times due to the cross sections being dependent on the simulation model. This technique will be described in the next section.

\subsubsection{Weighting Program}

To accomplish the goal of weighting events by the cross sections a program was written in Java that takes input events in a plain text file and then weights them according to cross sections that are also an input and then outputs the events after weighting. This is accomplished using rejection sample, where events are thrown away probabilistically in accordance with the input distributions.

The input events to the weighting program were three-body phase space events using the PLUTO generator. The generator weights events so that the distribution of the $\cos \left(\theta_{c m}\right)$ is flat from $[-1,1]$. This distribution allows an accurate reproduction of the input distributions by populating all regions of phase space equally. An improvement to the weighting program would be to directly incorporate the three-body generator directly into the weighting program so that an external generator is not needed and it would consume less computing resources. 
Due to the ambiguity of having two protons in the final state $\frac{d^{2} \sigma}{d M(p p) d \cos \left(\theta_{\bar{p}}^{*}\right)}$ was used as the central component for the generator (Fig. 4.1). This turned out to not be enough for the distributions to match between experiment and simulation so photon flux normalized $t_{\text {baryon }}, M\left(p_{\text {fast }} \bar{p}\right)$, and $\cos \left(\theta^{*}\right)$ of the slow proton were added as one dimensional profiles. Ideally the additional distributions would not have been added as one dimensional profiles but instead added as additional dimensions for the cross section, however this requires higher statistics than the experimental data could provide.

When weighting events the prior distribution of the events must be known so that you can normalize the probability that that event will be rejected by this distribution (Fig. 4.2). When the prior distribution is flat this is a simple task, however when weighting with multiple profiles and a dimensional cross section the prior distributions are dependent on one another. This was resolved using many iterations inside of the generator where the prior distribution of one variable was determined by weighting with all of the other variables. This was repeated until the weighted events matched the input distributions well (Fig. 4.3.

In order to reproduce the input distribution the true distribution that is being used to weight events is the input distribution divided by the prior distribution. Once you have the distribution that you will weight the events with you should also throw away a certain fraction of them in order to accurately reproduce areas of high probability.

\subsubsection{Quality Control}

In order to judge the quality of how the simulation matches the data comparison of all angles, momentum, invariant mass, and t distributions were used. Since 
the true distributions are not known then the distributions of the experimental data and the events that are accepted after being put into the detector simulation package must be compared. The comparisons between data and the simulated data that are discussed in this section are all normalized to the integral of the data histogram so that the shapes can be compared without the overall normalization interfering with the interpretation.

The distributions that are shown in Figures $4.4-4.9$ had the most difficulty in matching up with experimental data. The beam energy distribution was weighted as a one dimensional profile after the two dimension cross section was weighted, it shows good agreement with experimental data (Fig. 4.4). The dips in the beam energy spectrum due to inefficient tagger paddles. The dips caused by the inefficient tagger paddles is taken into account in the flux normalization.

The angular distributions were one of the most difficult distributions to reproduce. The angular distribution of the antiproton in the C.M. frame is weighted as a part of the two dimensional cross section $\frac{d^{2} \sigma}{d M(p p) d \cos \left(\theta_{\bar{p}}^{*}\right)}$. The accepted and experimental data show good agreement with some slight mismatch in the forward angles that could be addressed by interpolating the weighting histograms (Fig. 4.5). Additionally, as a part of the two dimensional cross section the bins are much courser, which can be seen as the steps in the generated histogram in blue.

The slow and fast proton proton distributions match well with data (Figs. 4.6 and 4.7). The fast proton distribution has an interesting dip that appears after applying the time-of-flight knockout cuts. Once the angular distributions of the antiproton and the slow proton are used in the weighting procedure the angular distribution of the fast proton matches up as a result.

Additionally, Mandelstam $t$ distributions are also shown for meson and baryon 
exchange models (Figs. 4.8 and 4.9 ). The baryon exchange model was used in the weighting process and it was one of the final distributions needed in order to match the data. Both $\mathrm{t}$ distributions match experimental data well.

Once the data matches the simulated events in all of the kinematic variables listed above then the process of adding additional distributions is finished and the process of assessing the change between simulation rounds starts. The cross section measurements are compared between simulation rounds and once the difference becomes smaller than the statistical uncertainty of the measurements then the process is complete. In total there were 17 rounds of simulation needed to get the number of distributions to match as well as having the model converge.

\subsubsection{Technical Considerations}

Initially several different computational methods were attempted including several generators and a program called PLUTO++ which allows the weighting of distributions by loading histograms. Using PLUTO++ with a one dimensional cross section $d \sigma / d \cos \left(\theta_{\bar{p}}^{*}\right)$ binned in photon beam energy did not prove to be satisfactory, showing that the reaction cannot be simply described by the angular distribution of one of the particles. Eventually when attempting to add more distributions to the generator it became apparent that more control was needed over the weighting process. The complexity of the number and types of distributions that needed to be included in the model led to the writing of this generator.

As a software developer for the next generation software for the upgrade to CLAS which is CLAS12 the choice to use the newer framework was justified by a shorter development cycle. The CLAS12 detector is similar to CLAS in it's 
physics goals and torus coil based design, however only the software framework will be discussed here. The CLAS12 software framework is largely based on Java which allows quick development of object oriented packages that can run on any machine that has a java virtual machine.

The parts of the CLAS12 framework that were used heavily in the development of this weighting program are GROOT, GAMP readers and writers, and physics libraries. GROOT is a plotting and fitting package that allowed for normalizing the histograms to produce a probability distribution used in the weighting process, it was developed at Jefferson Lab for CLAS12 and was a crucial piece of the weighting program. In order to save time PLUTO++ was used to generate 3-body phase space events which were saved to a GAMP format. The GAMP format is a simple text format used to save the four vector information and the particle type. This GAMP file was read in using existing libraries in CLAS12 software. The computations required to perform Lorentz boosts, rotations, and other operations were also provided in the CLAS12 software package.

Overall, with many dimensions and iterations required, it is computationally and memory intensive due to the many iterations of the input events required to fill the caches and the many histograms that are stored in memory. The time needed to weight the events is on the same order of magnitude with the time it takes GSIM and the reconstruction program to reconstruct the events. 

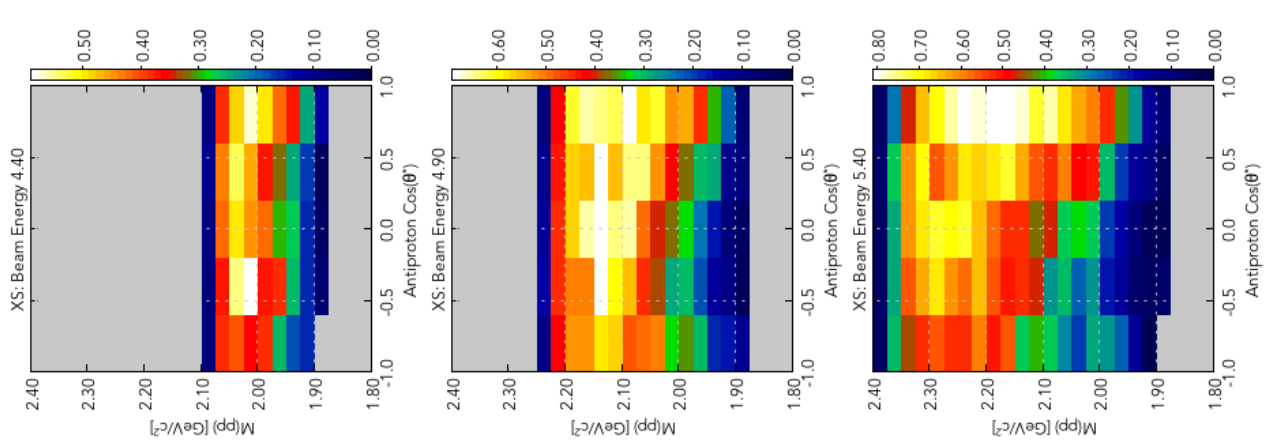

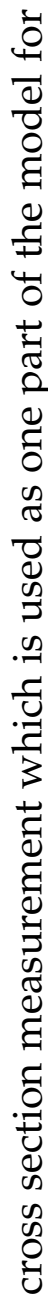
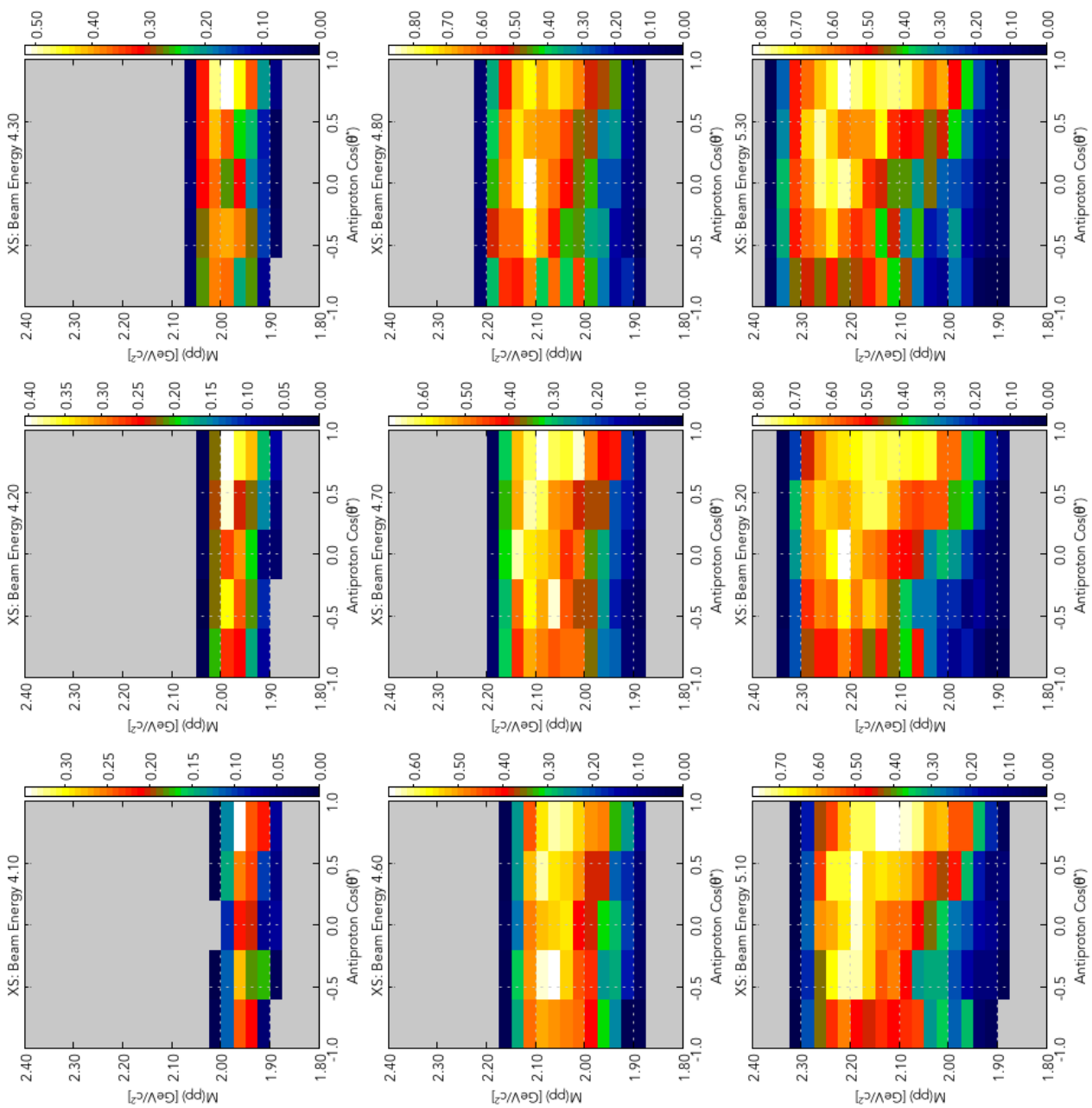

$\pi{ }^{2}$
0
0
0
0
0
0
0
0
0
0
0
3
3
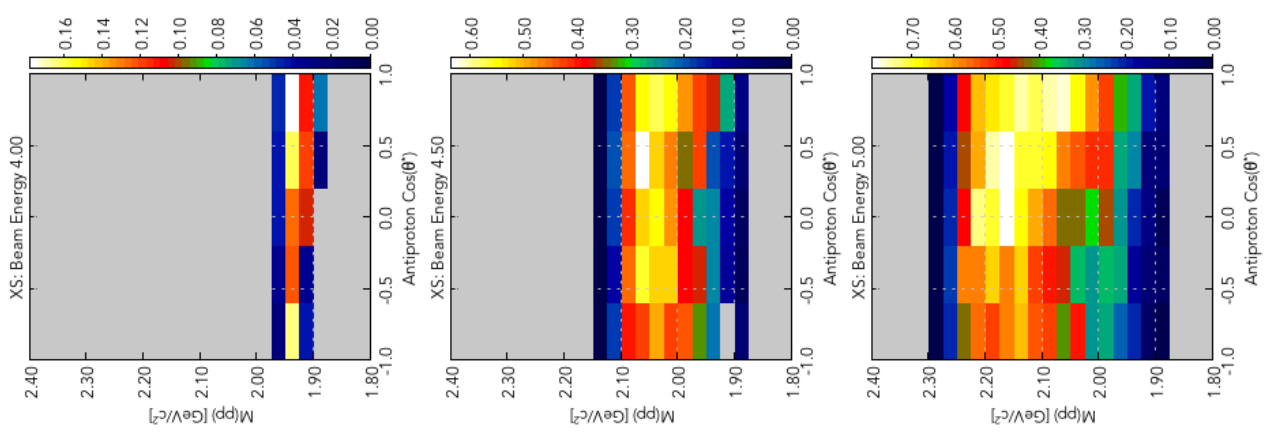

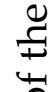

पै

$\sum_{\substack{\pi \\ 2}}^{\pi}$

$\sum$

范苋

ठ

类 

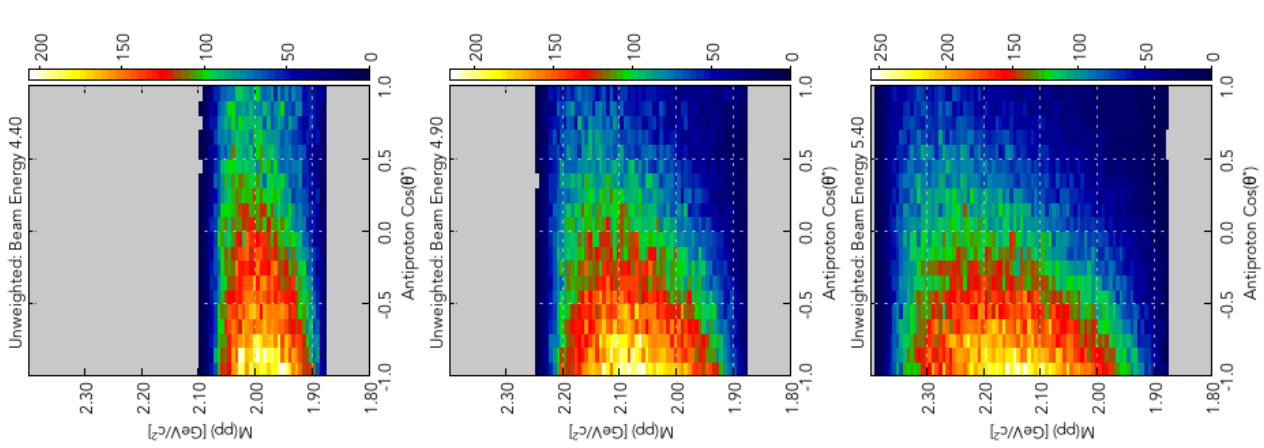

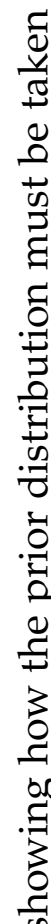
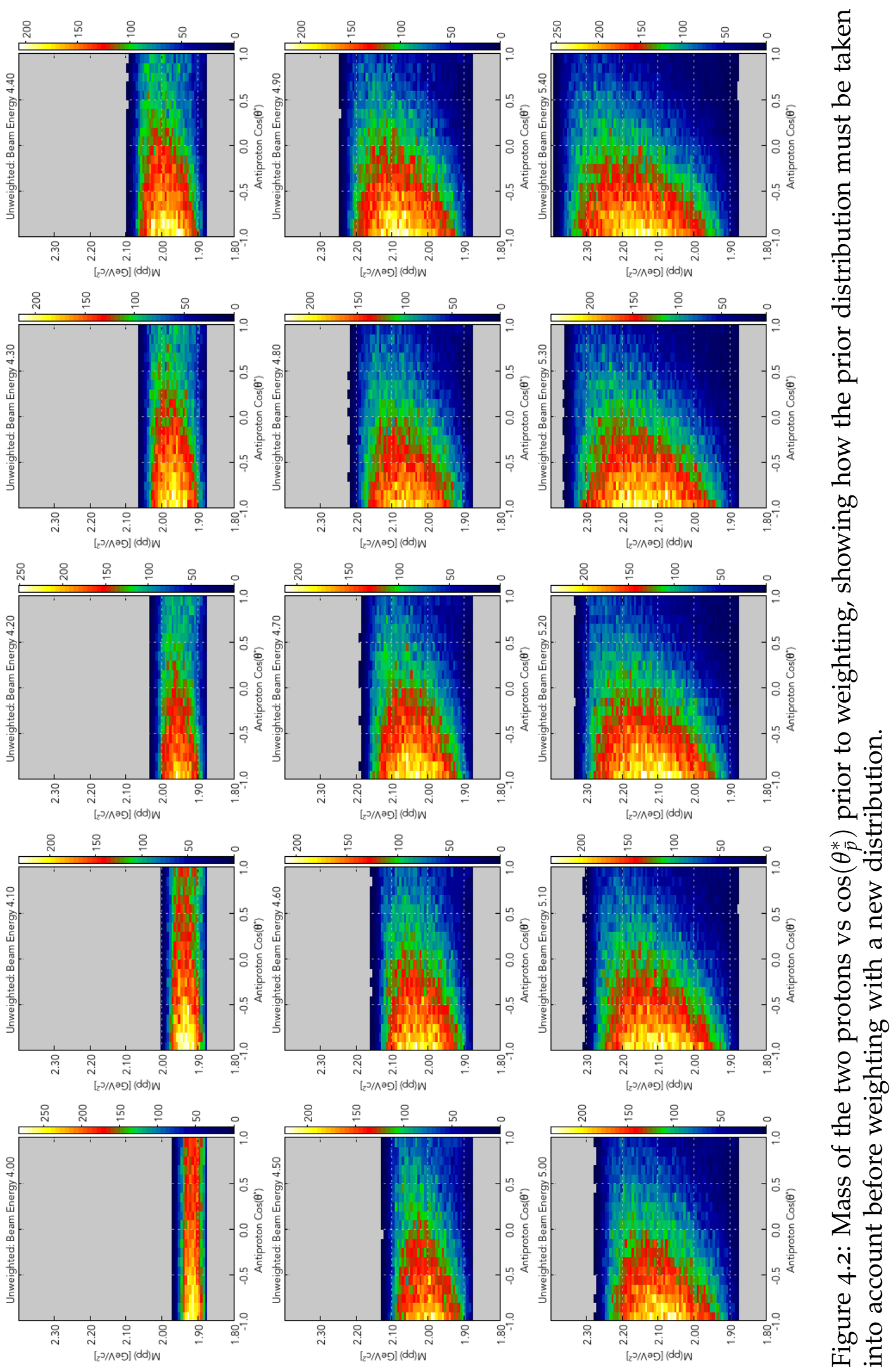

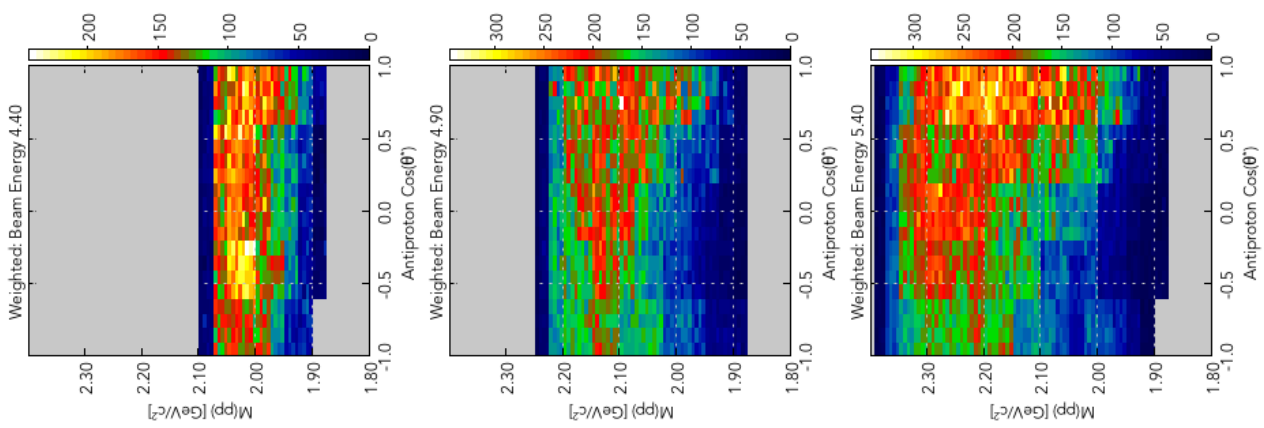

ํㅗㄹ
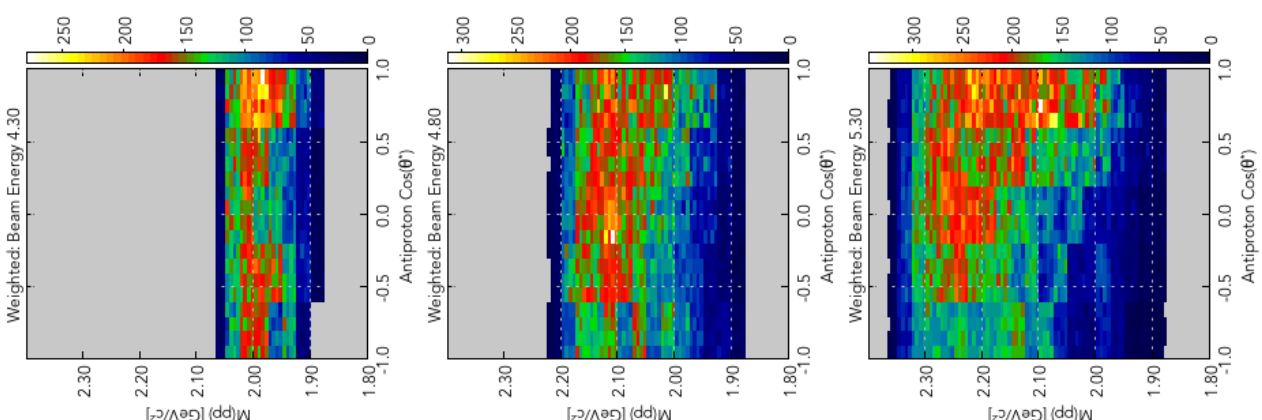

롬
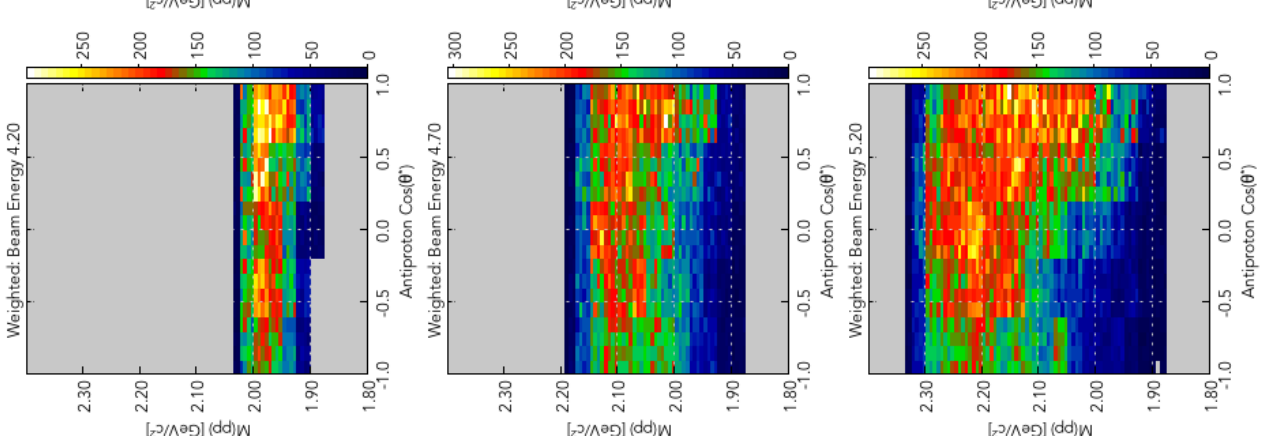

है
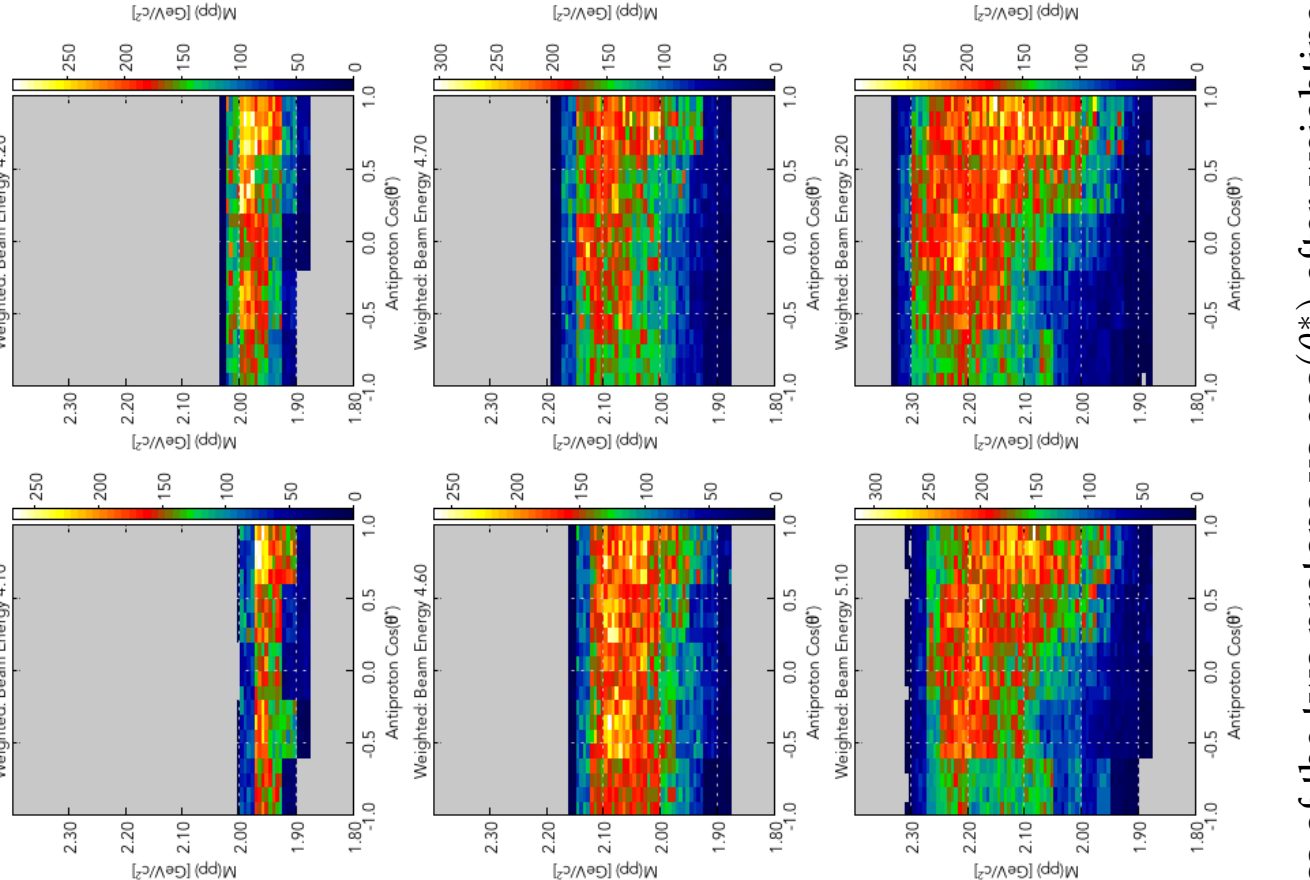

1

$\overbrace{0}^{* 12}$
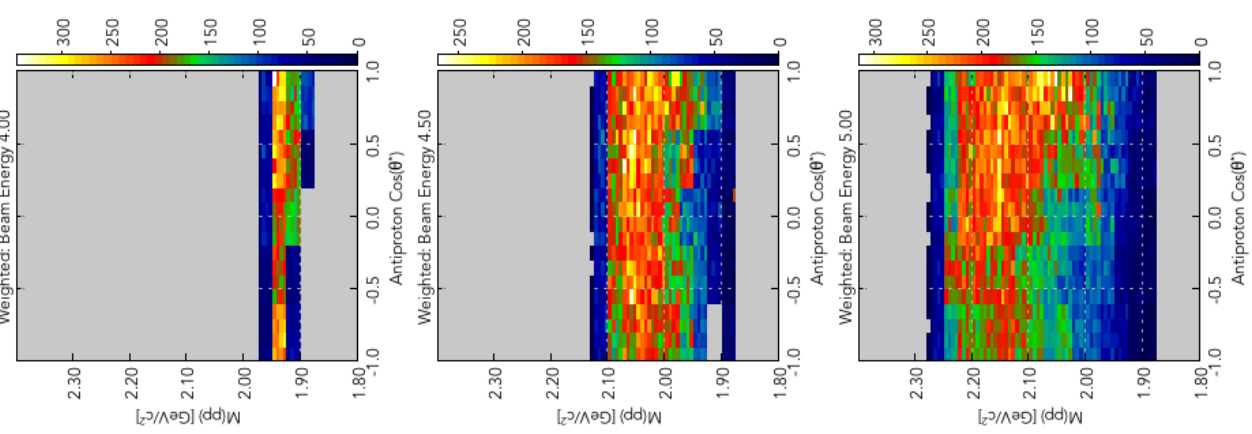

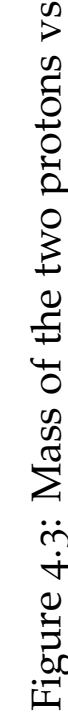




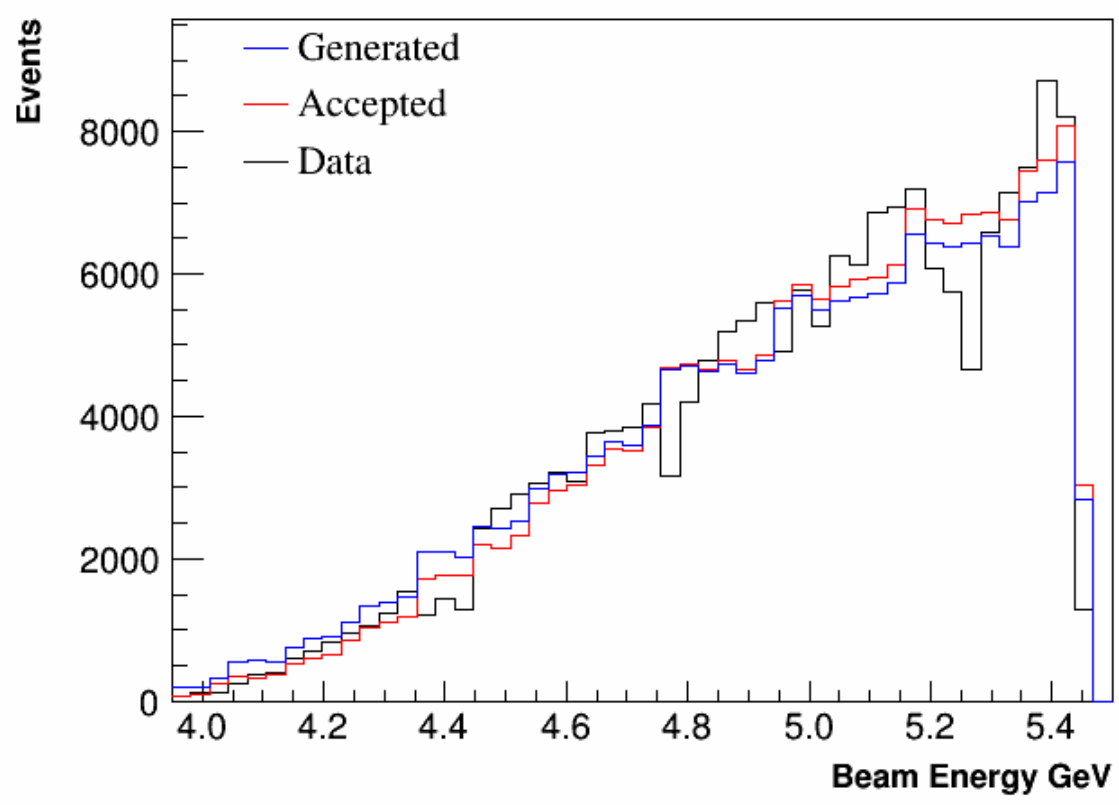

Figure 4.4: Photon beam energy distribution comparison between Monte Carlo events and experimental data.

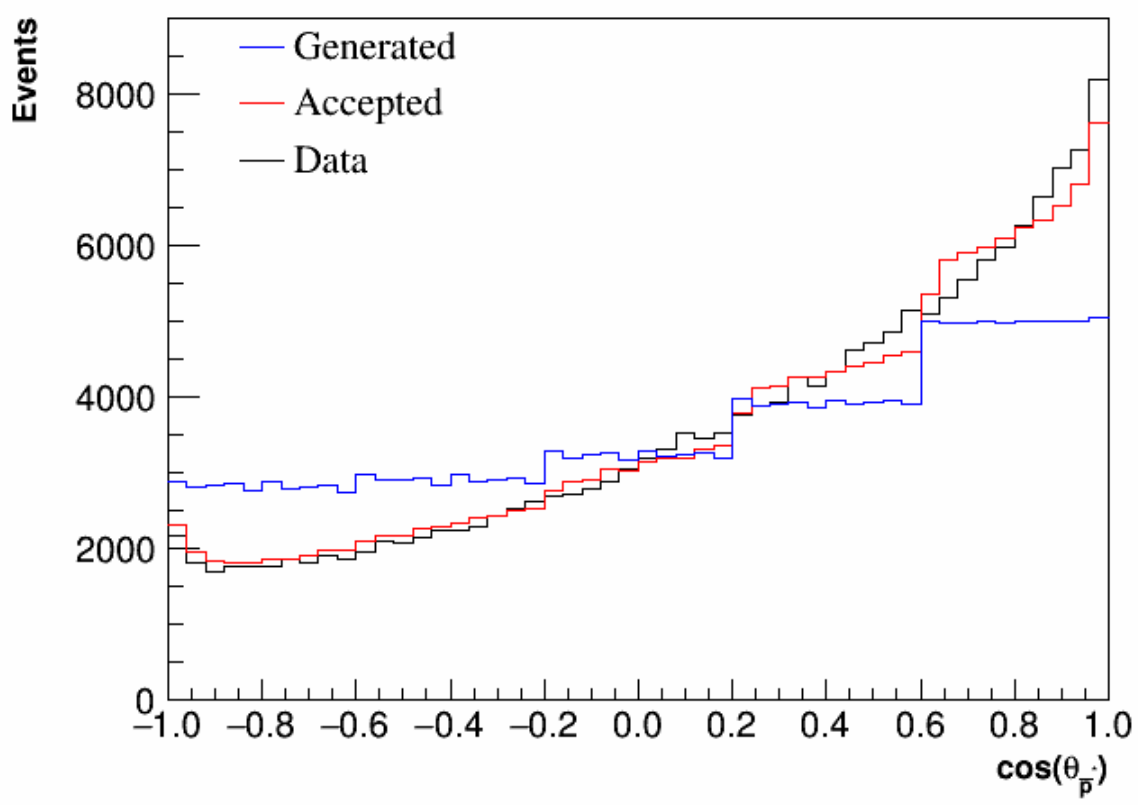

Figure 4.5: Angular distribution of the antiproton comparison between Monte Carlo events and experimental data. 


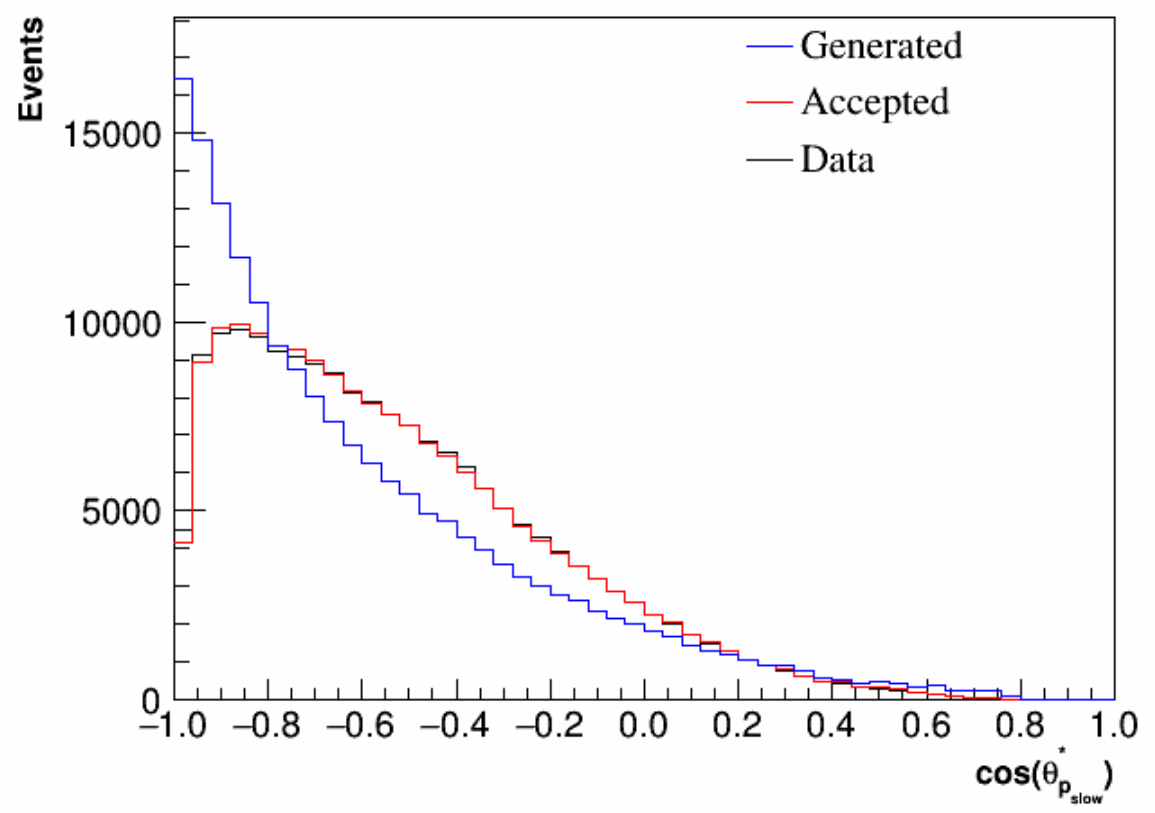

Figure 4.6: Angular distribution of the slow proton comparison between Monte Carlo events and experimental data.

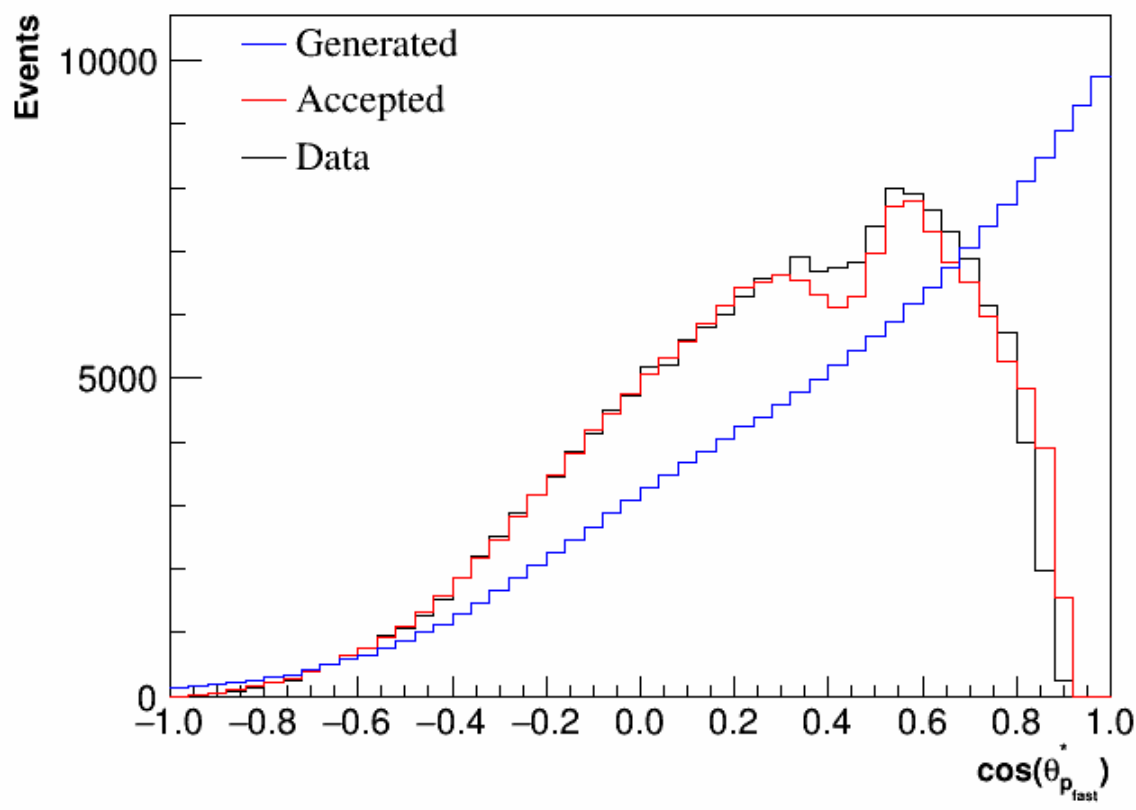

Figure 4.7: Angular distribution of the fast proton comparison between Monte Carlo events and experimental data. 


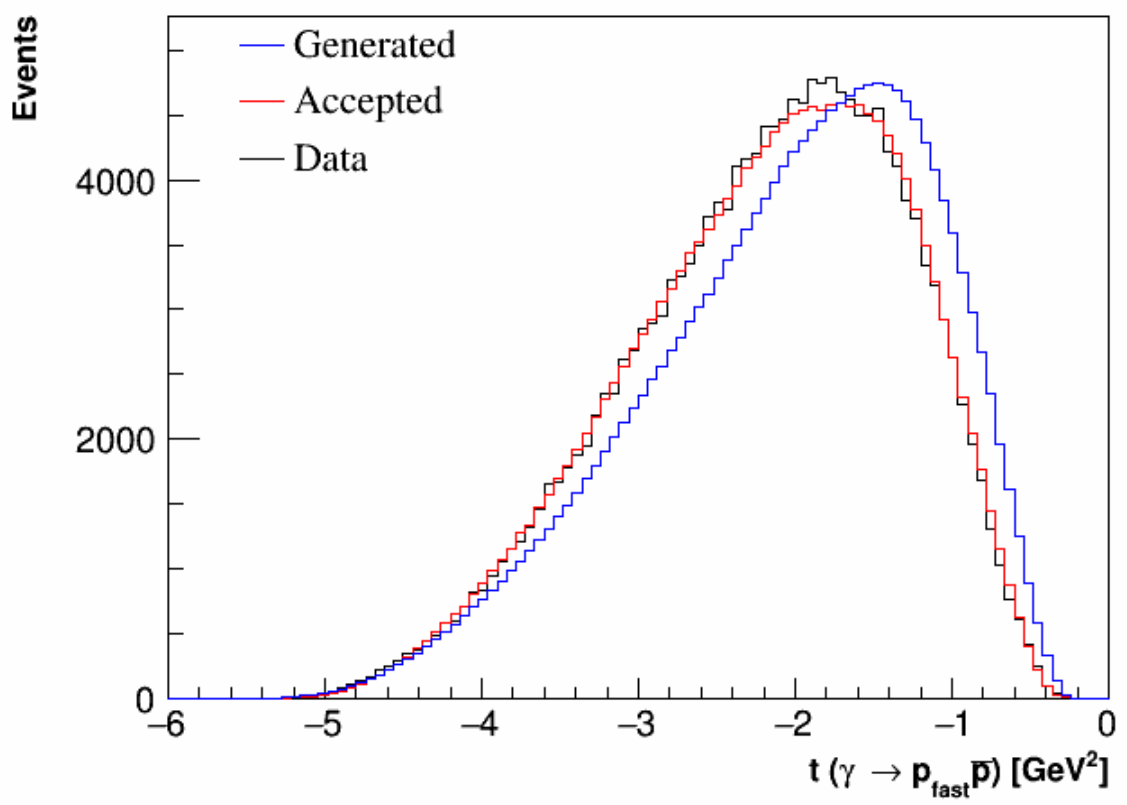

Figure 4.8: Distribution of t-meson comparison between Monte Carlo events and experimental data.

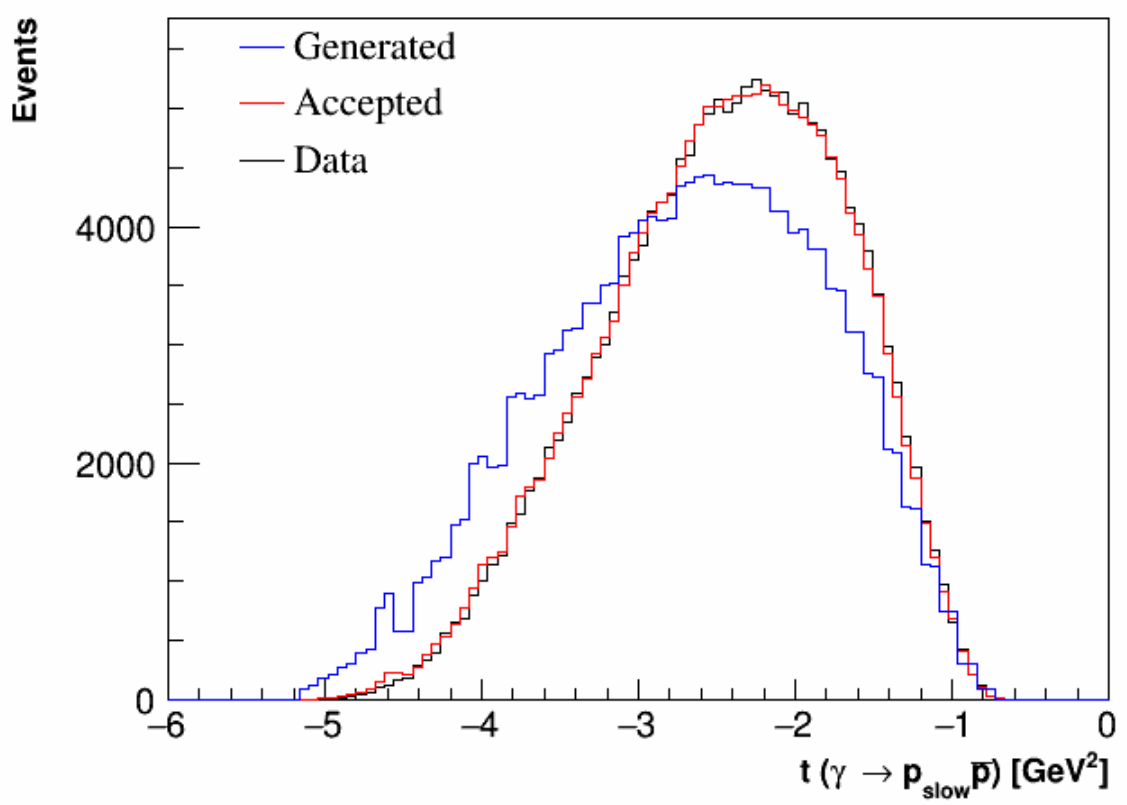

Figure 4.9: Distribution of t-baryon comparison between Monte Carlo events and experimental data. 


\subsubsection{Track Efficiency Correction}

The purpose of the track efficiency correction is to take into account the differences in reconstruction efficiency between the simulated data and experimental data. The track efficiency correction is determined by using a topology that utilizes missing mass to reconstruct a track. The track efficiency is the ratio of number of events reconstructed with a missing particle and how many of those events had the missing particle reconstructed as a track. This study is done for experimental data and simulated data and the difference is used as a correction factor.

These track efficiency corrections were performed using the reaction $\gamma p \rightarrow$ $p \pi^{+} \pi^{-}$by Michael Kunkel. The track efficiency was calculated independently for each particle in bins of the z-vertex position in the target, the momentum magnitude and direction. For the $\gamma p \rightarrow p p(\bar{p})$ reaction the only particles being reconstructed are protons which allows the standard $g_{12}$ track efficiency to be used. In future analysis an independent track efficiency will be derived for antiprotons.

\subsection{Differential Cross Sections}

We have measured $d \sigma / d \cos \left(\theta_{\bar{p}}\right), d \sigma / d M(p p), d \sigma / d M(p \bar{p})$ for the first time. For calculating the cross section we use kinematic fitting to determine the yields of the data and the reconstructed Monte Carlo data.

\subsubsection{Mass Distributions of Events}

A first measurement of $d \sigma / d M(p p)$ and $d \sigma / d M(p \bar{p})$ have been made. Due to the ambiguities in distinguishing between which proton was produced and which 
one was the recoil proton the $d \sigma / d M(p \bar{p})$ is double counted as it is a combination of $p_{1} \bar{p}$ and $p_{2} \bar{p}$ pairs. The differential cross sections were measured using $100 \mathrm{MeV}$ steps in photon beam energy from 3.95 to $5.45 \mathrm{GeV}$. Each measurement consists of 45 bins in $M(p p)$ or $M(p \bar{p})$ from 1.8 to $2.4 \mathrm{GeV} / c^{2}$ shown in Fig. 4.10 .

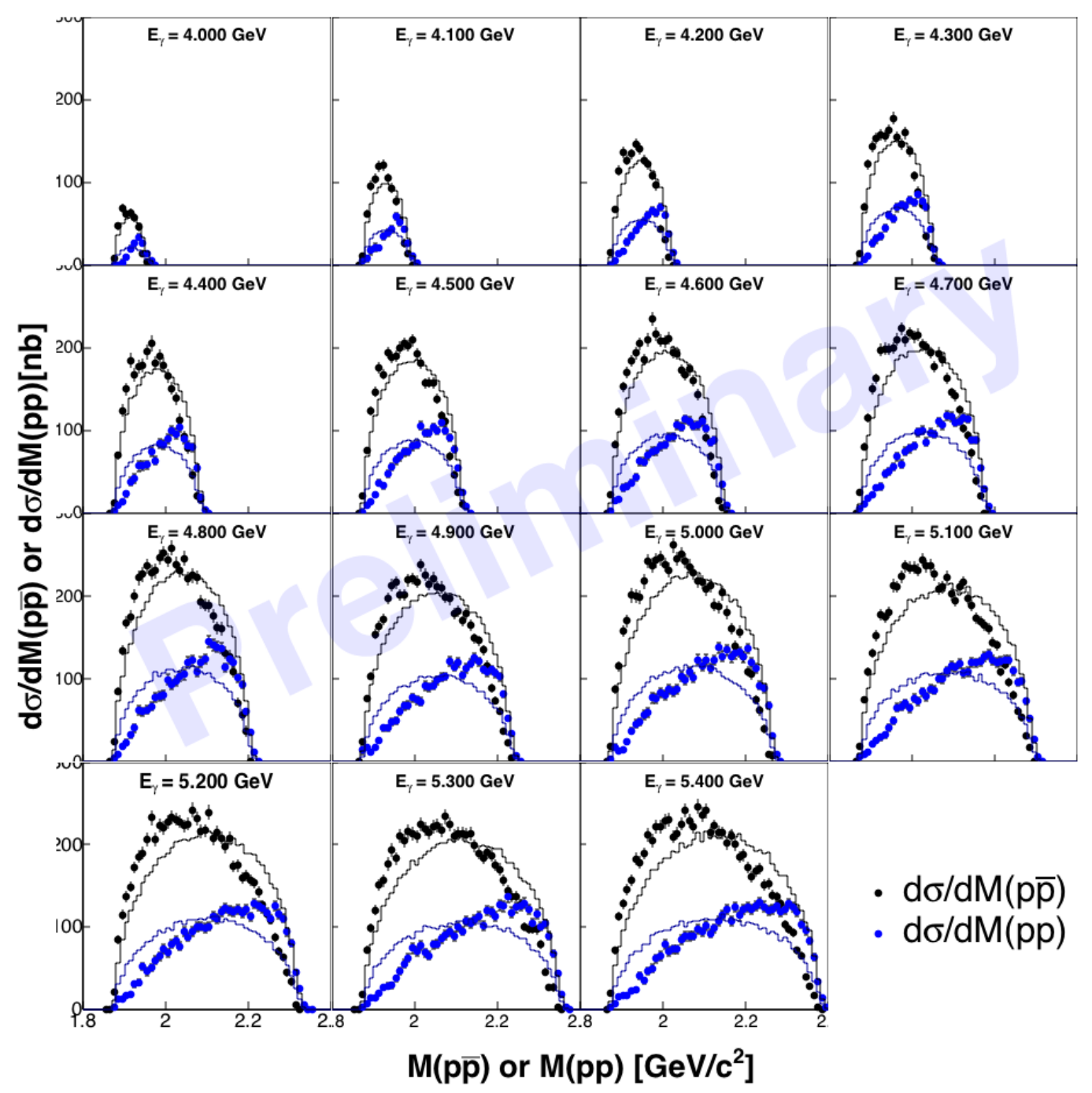

Figure 4.10: Differential cross section for $\gamma p \rightarrow p p \bar{p}$ showing the mass distributions as a function of beam energy. Histograms showing the phase space mass distributions are also shown in the same color as the graphs. Only statistical uncertainties are shown. 


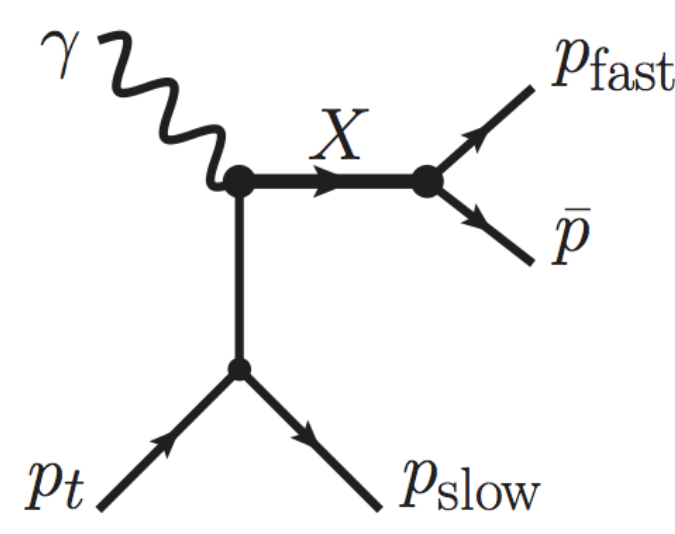

(a) t-channel

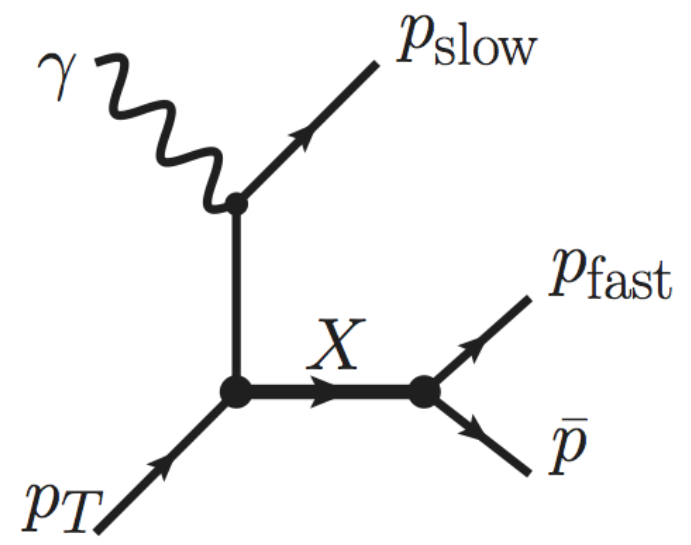

(b) u-channel

Figure 4.11: Feynman diagrams showing $p \bar{p}$ production models with a meson exchange model ( $\mathrm{t}$-channel) on the left and a baryon exchange model ( $\mathrm{u}$-channel)

\subsubsection{Angular Distributions of Events}

The measurement of the angular distribution of the events can provide information about the production mechanism. There are thought to be two main contributions as seen in Figure 4.11 a meson exchange process that would send the $p \bar{p}$ in a more forward direction and a baryon exchange model where the $p \bar{p}$ pair would favor a backward direction (Fig. 4.11).

Ideally the produced $p \bar{p}$ angular distribution would be studied but due to the two identical particles in the final state this could not be done. The only way to measure the differential cross section without the ambiguity is to use the antiproton angular distributions. The differential cross section $d \sigma / d \cos \left(\theta_{\bar{p}}\right)$ was measured from 3.95 to $5.45 \mathrm{GeV}$ in $50 \mathrm{MeV}$ bins in photon energy and 10 bins in $\cos (\theta)$ of the antiproton for a total of 300 measurements. Due to not having to detect the antiproton there is coverage over the full angular range which is shown in Figures 4.12 and 4.13 .

The angular distribution shows an excess in the forward and backward regions in all beam energy bins. In order to quantify the shape a legendre poly- 
nomial fit was used and it shows that there is more deviation from a uniform distribution seen in lower energy bins Figs. 4.14 4.15

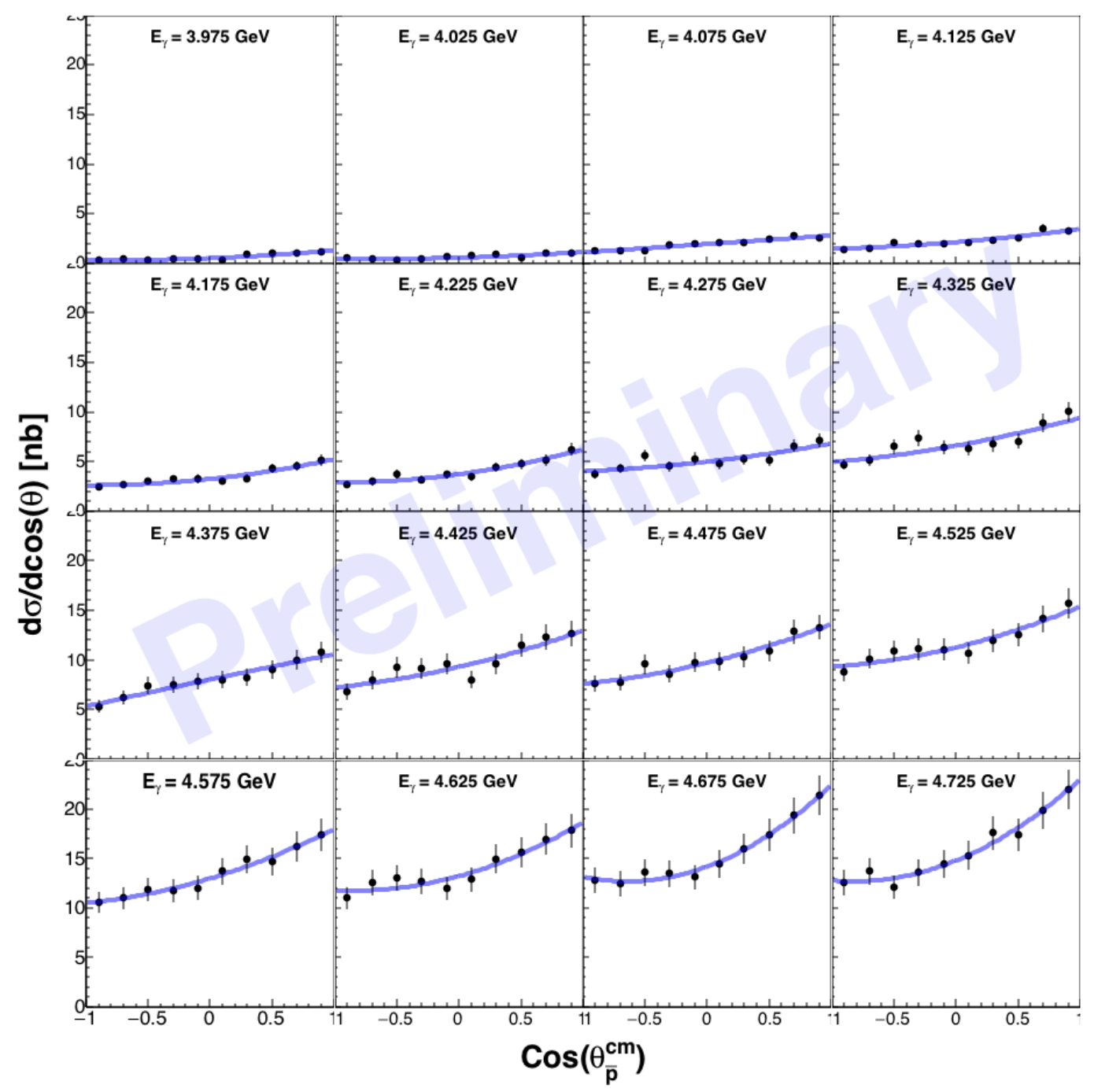

Figure 4.12: First measurement of the differential cross section for $\gamma p \rightarrow p p \bar{p}$ showing the angular distribution of the antiproton in the center of momentum frame as a function of beam energy. The fits are to Legendre polynomials 


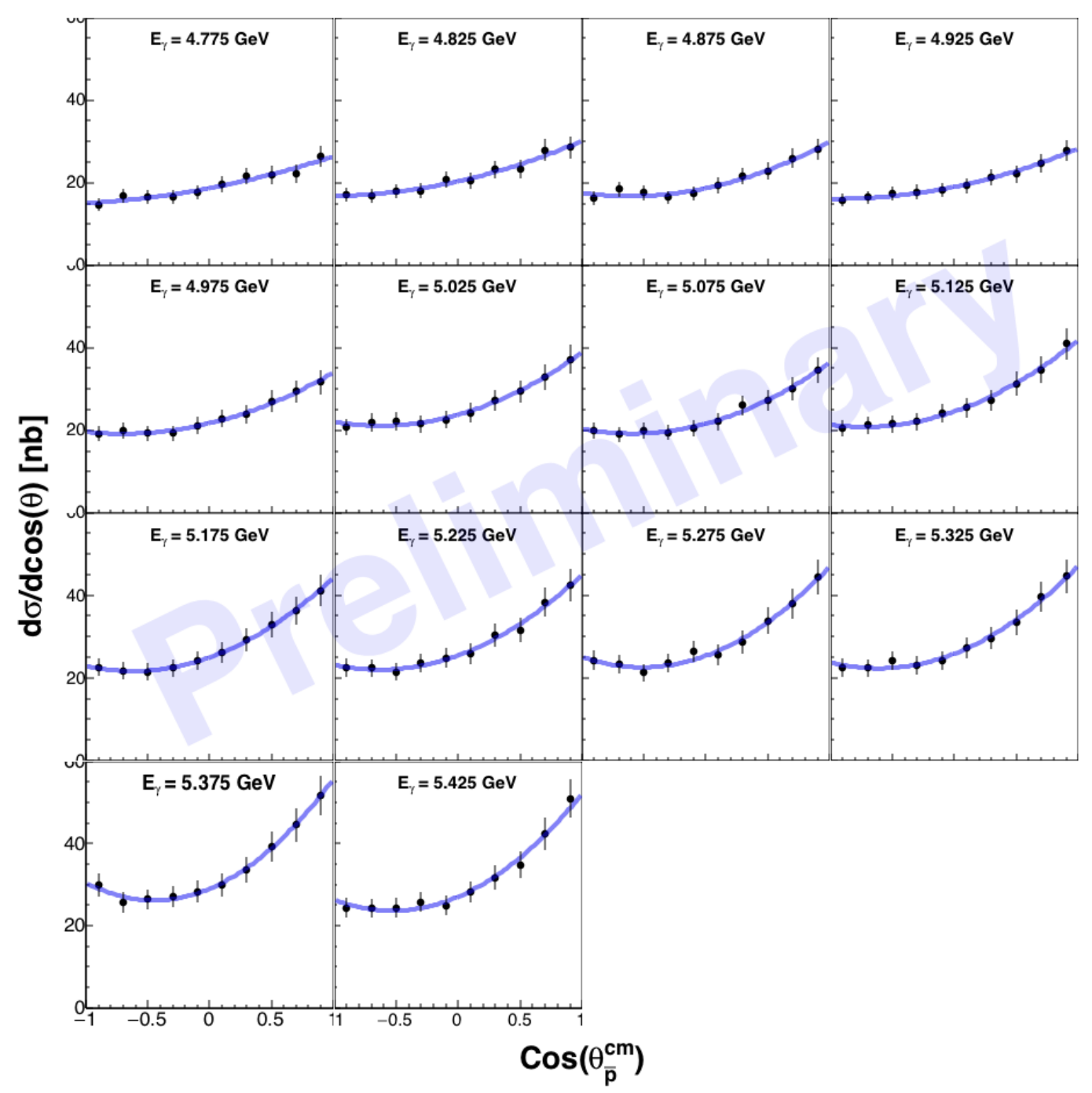

Figure 4.13: First measurement of the differential cross section for $\gamma p \rightarrow p p \bar{p}$ showing the angular distribution of the antiproton in the center of momentum frame as a function of beam energy. The fits are to Legendre polynomials. 


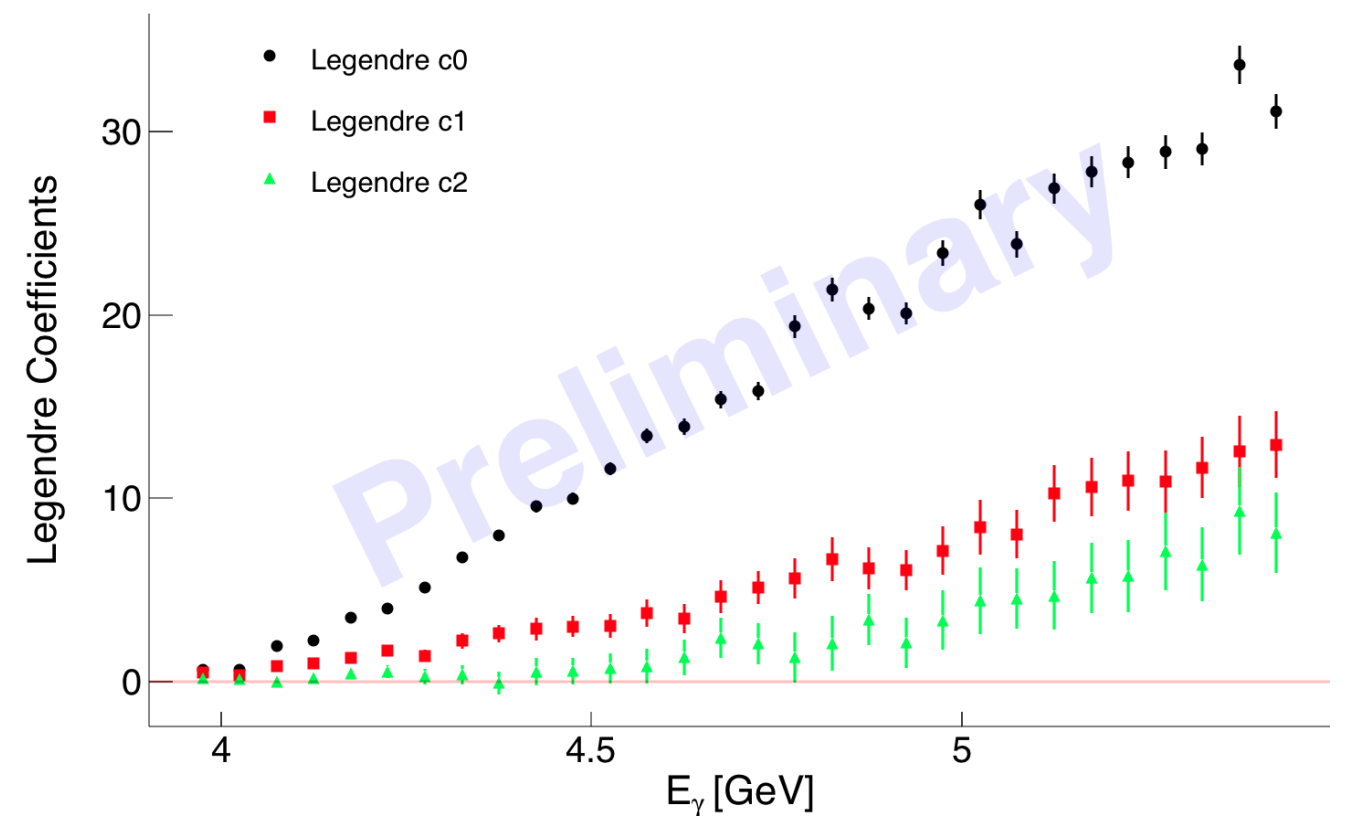

Figure 4.14: Legendre polynomial coefficients for $d \sigma / d \cos \left(\theta_{\bar{p}}\right)$.

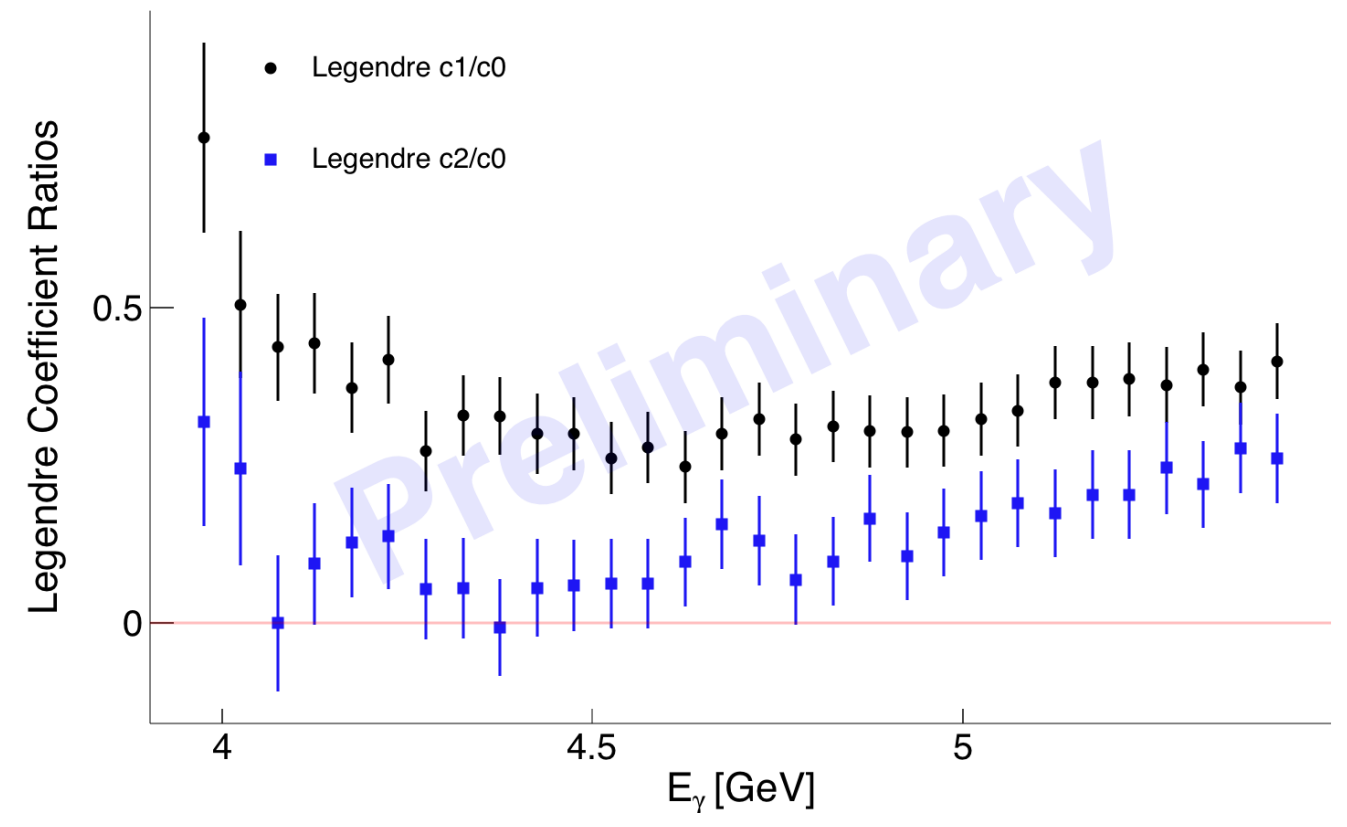

Figure 4.15: Legendre polynomial coefficient ratios from the fit to $d \sigma / d \cos \left(\theta_{\bar{p}}\right)$. The ratio shows how the shape of the differential cross section varies with beam energy $d \sigma / d \cos \left(\theta_{\bar{p}}\right)$. Overall the angular distribution becomes more uniform as the photon energy increases. 


\subsection{Total Cross Section Measurement}

In comparison with previous experiments we have measured the total cross section of the reaction $\gamma p \rightarrow p p \bar{p}$ from $E_{\gamma}$ from $3.95 \mathrm{GeV}$ to $5.45 \mathrm{GeV}$. Each measurement is from integrating the Legendre polynomial fit to the differential cross section, $d \sigma / d \cos \left(\theta_{\bar{p}}\right)$ (Fig. 4.16). As shown in the table, this experiment has the largest statistics by far and was the first to be able to integrate differential cross sections in order to calculate the total cross section. This technique of integrating the differential cross sections may help mitigate the Monte Carlo model dependence of the acceptance.

\begin{tabular}{c|c}
\hline Experiment & Number of Events \\
\hline \hline DESY & 200 \\
LAMP2 & 137 \\
CLAS g6b & 2,500 \\
CLAS g6c & 25,000 \\
CLAS g12 & 250,000 \\
\hline
\end{tabular}

\subsection{Cross Sections with $\gamma p \rightarrow p \bar{p}(p)$}

As a cross check the same differential cross sections and total cross section measurements have been calculated using the missing proton dataset. The difficulties in using this reaction as a cross check are a result of the lower statistics and the hole in acceptance of the antiproton due to the holes in the tracking acceptance of CLAS for particles that are bent toward the beam line. The total cross section measurement shows a good agreement with previous experimental data in Fig. 4.17. 


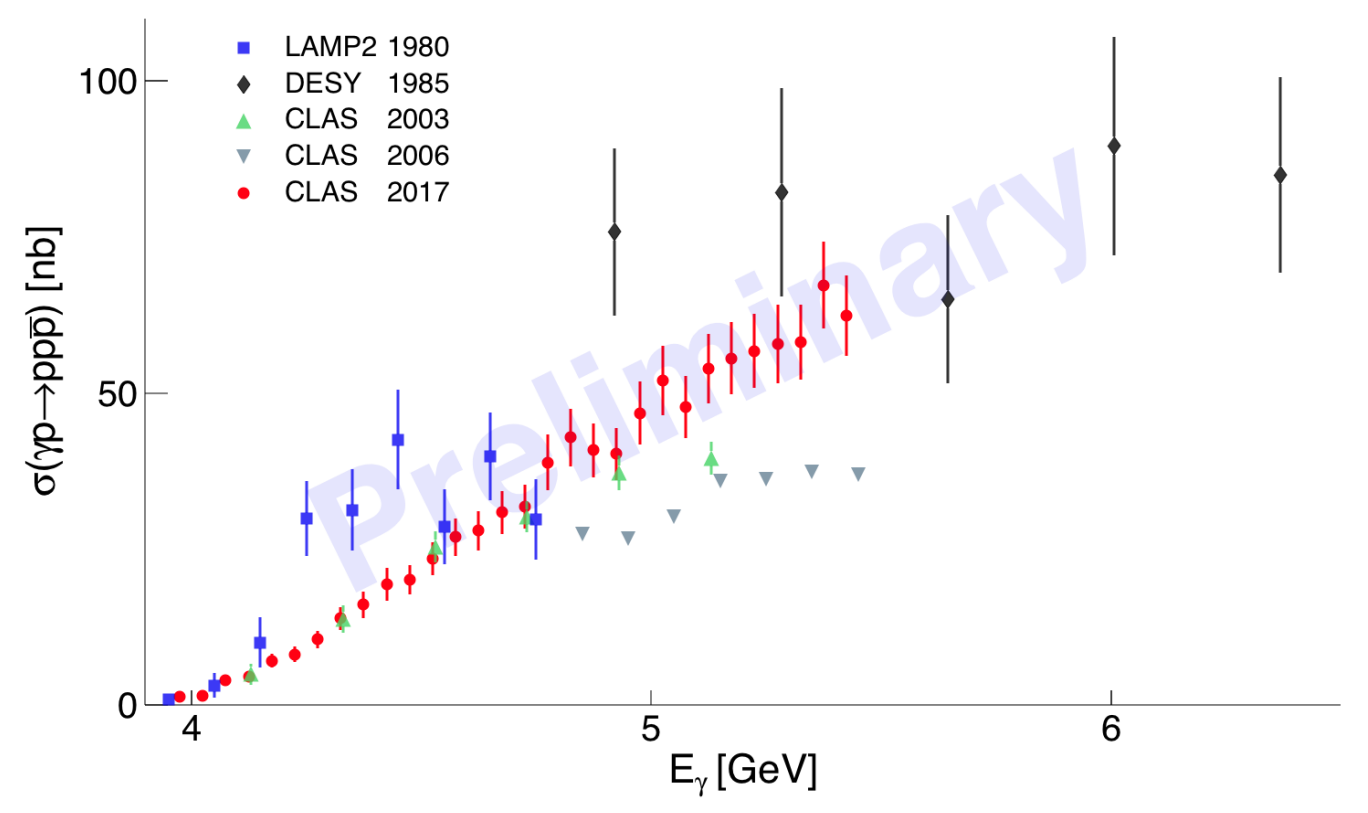

Figure 4.16: Total cross section for $\gamma p \rightarrow p p \bar{p}$ comparing the measurement that is this work to existing world data from DESY and LAMP2 [27] [8].

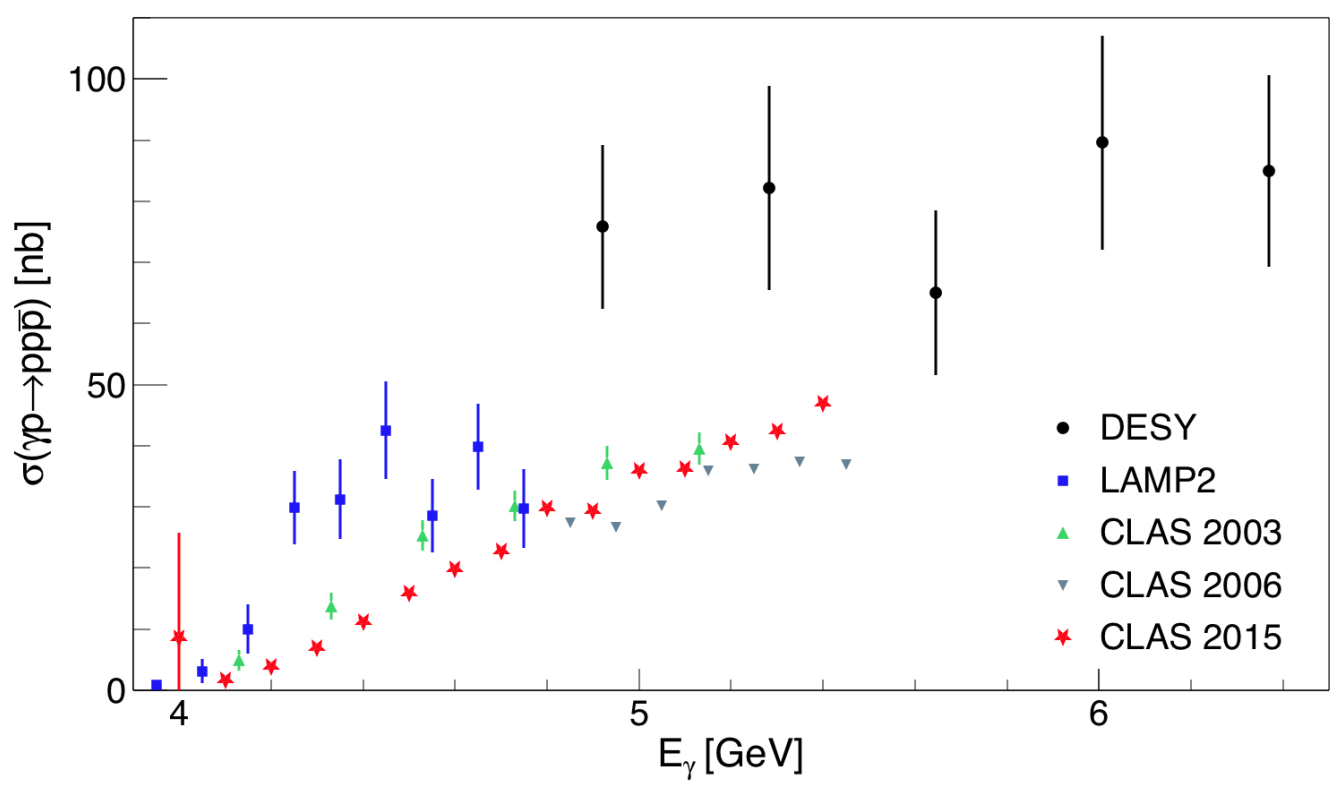

Figure 4.17: Total cross section for $\gamma p \rightarrow p p \bar{p}$, calculated using the missing proton data, comparing the measurement that is this work to existing world data from DESY and LAMP2 [27] [8]. Only statistical uncertainties are shown. 


\subsubsection{Comparison with Theory}

Recent theoretical work has been done to predict the cross sections of the reaction $\gamma \rightarrow p p \bar{p}$ [28]. The current model which takes into account two multi-

plets of scalar mesons, $f_{0}(1370)+f_{0}(1500)+f_{0}(1710)$ and $f_{0}(2020)+f_{0}(2100)+$ $f_{0}(2200)$ calculations fall well below the measurements from our data and is shown in Figure 4.18. The Full Regge model shown uses known photo-couplings for the lower mass multiplet and an increased estimate of the photo-coupling for the higher mass, even with the increased photo-coupling the prediction is significantly lower in the lower photon energy range and about half of the total cross section at higher photon energies.

\section{$4 \cdot 7$ Uncertainty}

There are two types of uncertainty that are calculated for these measurements. The first is the statistical uncertainty relating to the total number of events in each measurement and other count-related quantities such as the photon flux. The second type of uncertainty is in the process of measuring these numbers and is called systematic uncertainty.

In the following text and equations the cross section will be defined as $\sigma, \mathrm{Y}$ as the yield, and $\epsilon$ for the detector efficiency.

\subsubsection{Statistical Uncertainty}

The statistical uncertainty for these measurements is based on Poisson statistics giving $\delta_{N}=\sqrt{N}$. Where the statistical uncertainty on each cross section 


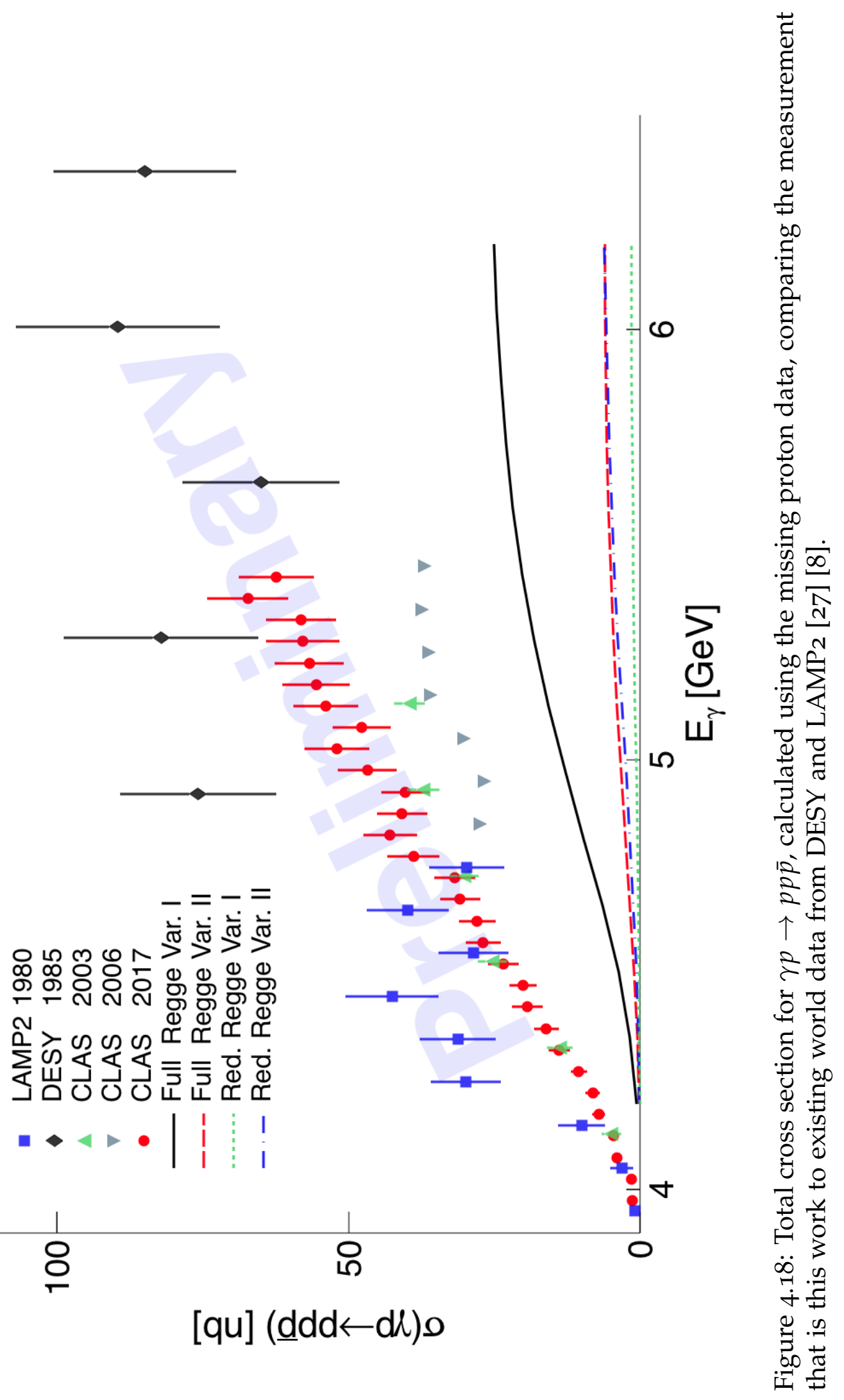


measurement is

$$
\delta_{\sigma}=\sigma \cdot \sqrt{\left(\frac{\delta_{\mathrm{Y}}}{\mathrm{Y}}\right)^{2}+\left(\frac{\delta_{\epsilon}}{\epsilon}\right)^{2}+\left(\frac{\delta_{f l u x}}{N_{f l u x}}\right)^{2}} .
$$

The statistical uncertainty for the detector efficiency, $\epsilon$ (Eqn. 3.2) is calculated as

$$
\delta_{\epsilon}=\epsilon \sqrt{\frac{\epsilon(1-\epsilon)}{N_{g e n}}} .
$$

The statistical uncertainty of the photon flux $\sigma_{f l u x}$ is due to counting uncertainties and is defined as

$$
\delta_{f l u x}=\sqrt{N_{f l u x}}
$$

\subsubsection{Cut-based Systematic Uncertainty}

In order to quantify the systematic uncertainty associated with each cut, the cut will be varied and the difference in the measurement value will determine the systematic uncertainty. The reference cross section measurement is $\sigma_{r e f}$ and the cut variation is $\sigma_{v a r}$. The systematic uncertainty associated with the cut is defined as

$$
\delta_{c u t}=\frac{\left|\sigma_{r e f}-\sigma_{v a r}\right|}{\sigma_{r e f}} .
$$

The following cuts were studied: kinematic fit pull probability, fiducial cut, and target fiducial cut (Tab. 4.2.

\subsubsection{Model-Dependence of the Simulation}

Instead of varying the simulation parameters like the cut based uncertainty is calculated by looking at the difference between iterations of the simulation. The simulation is described thoroughly later in this chapter but it is based on using the cross sections as the input for the generator and it requires many iterations 
before converging. The systematic uncertainty for the simulation model where the current iteration is $\mathrm{n}$ is then defined as

$$
\delta_{\text {model }}=\frac{\left|\sigma_{n}-\sigma_{n-1}\right|}{\sigma_{n}} .
$$

\subsubsection{Sector-Dependent Systematic Uncertainty}

The CLAS detector is essentially six independent spectrometers with different calibrations and efficiencies. The two detected protons can be reconstructed from different sectors so requiring both to be in a single sector could bias the data. In this case it is better to remove one sector from the measurement requiring both protons be reconstructed in any sector but the one being studied. The differences in the cross section from the average of all 6 measurements with missing sectors is used as the systematic uncertainty. The sector based systematic uncertainty it then

$$
\delta_{\text {sector }}=\sqrt{\sum_{i=1}^{6}\left(\sigma_{i}-\mu\right)} .
$$

\subsubsection{Photon Flux Systematic Uncertainty}

The photon flux systematic was studied using the $\gamma p \rightarrow p \omega$, where $\omega \rightarrow$ $\pi^{+} \pi^{-} \pi^{0}$ since the omega photoproduction cross section is relatively high and the background is low. The normalized yield of the reaction $\gamma p \rightarrow p \omega$ was compared on run-by-run basis showing how the normalized yield varied throughout the experiment Fig. 4.19. This shows that there are no large deviations that are run dependent.

In order to check that the photon flux calculation is not beam current dependent each beam current setting was compared and fit with a first order polyno- 
mial Fig. 4.20. The fit results show a slope consistent with zero, showing that there is no overall beam current dependent systematic uncertainty. The outlying points at lower beam currents were not a significant portion of the production runs in Figure 4.20 .

In order to calculate the systematic uncertainty for the flux, the $60 \mathrm{nA}$ and $65 \mathrm{nA}$ beam currents are investigated since they are the beam current datasets with the highest statistics (Fig. 4.21). The normalized yield for each run is filled into a histogram for each current and the width of the distribution corresponds to the statistical uncertainty of the runs and the difference in means is directly related to the systematic uncertainty. The higher statistics $60 \mathrm{nA}$ run will be the reference measurement using the mean of the Gaussian fit $\mu_{60 \mathrm{nA}}$, and the $60 \mathrm{nA}$ Gaussian mean $\mu_{65} \mathrm{nA}$ run will be the variation in the measurement. The photon flux systematic is defined as

$$
\delta_{f l u x}=\frac{\left|\mu_{60 \mathrm{nA}}-\mu_{65 \mathrm{nA}}\right|}{\mu_{60 \mathrm{nA}}} .
$$

The resulting systematic uncertainty for the flux is 0.057 .

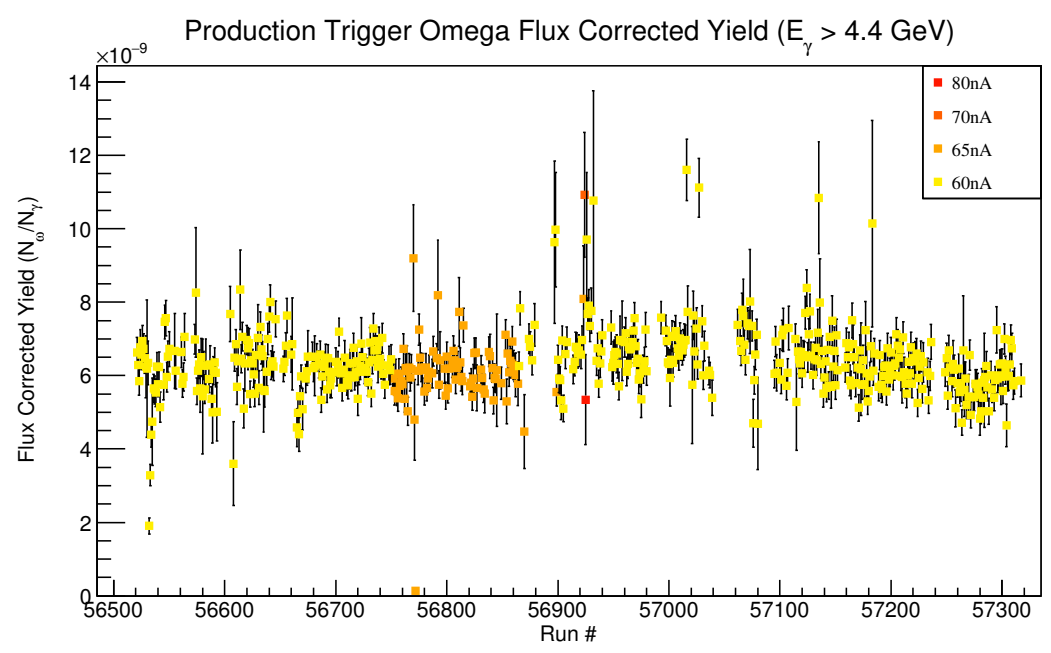

Figure 4.19: The run-by-run stability of the photon flux for all production runs excluding single track trigger runs. 


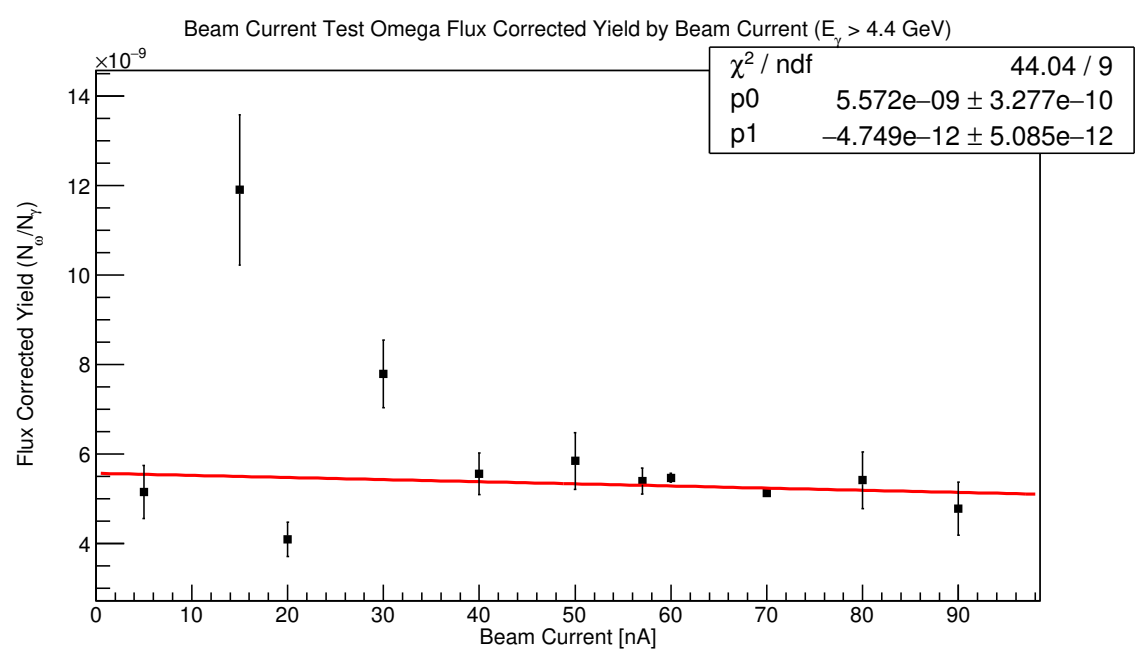

Figure 4.20: Comparison of the flux normalized yield for all beam currents, showing no beam current dependent systematic uncertainty with a slope consistent with o.

Production Trigger Beam Current Comparison

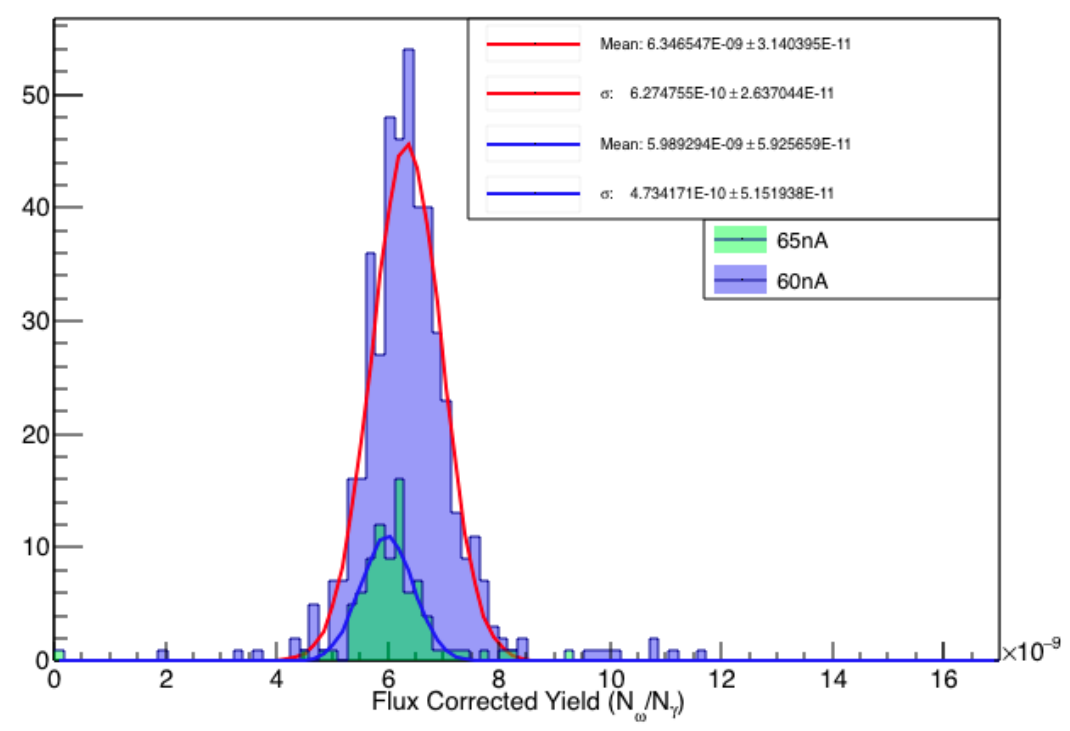

Figure 4.21: The flux normalized yield for the two highest statistics beam currents. The $\sigma$ is correlated with the statistical uncertainty and the difference in means is used as the systematic uncertainty. 


\subsubsection{Track Efficiency Systematic Uncertainty}

The track efficiency systematic uncertainty was studied using the reaction $\gamma p \rightarrow$ $p \pi^{+} \pi^{-}[29]$. The track efficiency was binned in $\theta$ and $\phi$ in the lab frame for each particle, and for more specifics see Chapter 3 . This binning was varied and used to calculate the track efficiency systematic uncertainty defined as

$$
\sigma_{\text {track }}=\frac{x_{\text {previous }}-x_{\text {new }}}{\frac{x_{\text {previous }+x_{\text {new }}}}{2}}
$$

for a three charged track final state. This is an overestimate since there were three charged tracks reconstructed but in this analysis only two protons were reconstructed. A conservative estimate averaging the photon energy dependence would yield a systematic uncertainty of $2.3 \%$.

\subsubsection{Systematic Uncertainty Summary}

The individual systematic uncertainty measurements are assumed to be uncorrelated and can be added in quadrature giving a total systematic uncertainty of

$$
\sigma_{\text {total }}^{\text {systematic }}=\sqrt{\sum_{n=1}^{n_{\text {sys }}} \sigma_{n}^{2}} .
$$


Table 4.2: List of systematic uncertainties.

\begin{tabular}{c|c} 
Systematic & Relative Uncertainty \\
\hline \hline Sector & 0.071 \\
Pull Probability & 0.033 \\
Track Efficiency & 0.020 \\
Monte Carlo Model & 0.022 \\
Fiducial Cut & 0.021 \\
Target Fiducial & 0.010 \\
\hline Total & 0.087
\end{tabular}

Table 4.3: List of global systematic uncertainties.

\begin{tabular}{c|c} 
Global Systematic & Relative Uncertainty \\
\hline \hline Photon Flux & 0.057 \\
Target Density & 0.025 \\
\hline Total & 0.062
\end{tabular}




\section{CHAPTER 5}

\section{Cross section measurement of $\gamma p \rightarrow p p \bar{n} \pi^{-}$}

\subsection{Overview}

In addition to the proton antiproton channel, antiprotons and antineutrons have been studied in the $N \bar{N} \pi$ reactions. The reactions, $\gamma p \rightarrow p n \bar{p} \pi^{+}$and $\gamma p \rightarrow$ $p p \bar{n} \pi^{-}$both have a yield of about 1200 events each. This is a first-time observation of an antineutron in photoproduction. This reaction was originally looking for $\Delta$ baryons however without the proper statistics the total cross section was measured and can provide valuable information about photo-couplings and for planning future experiments

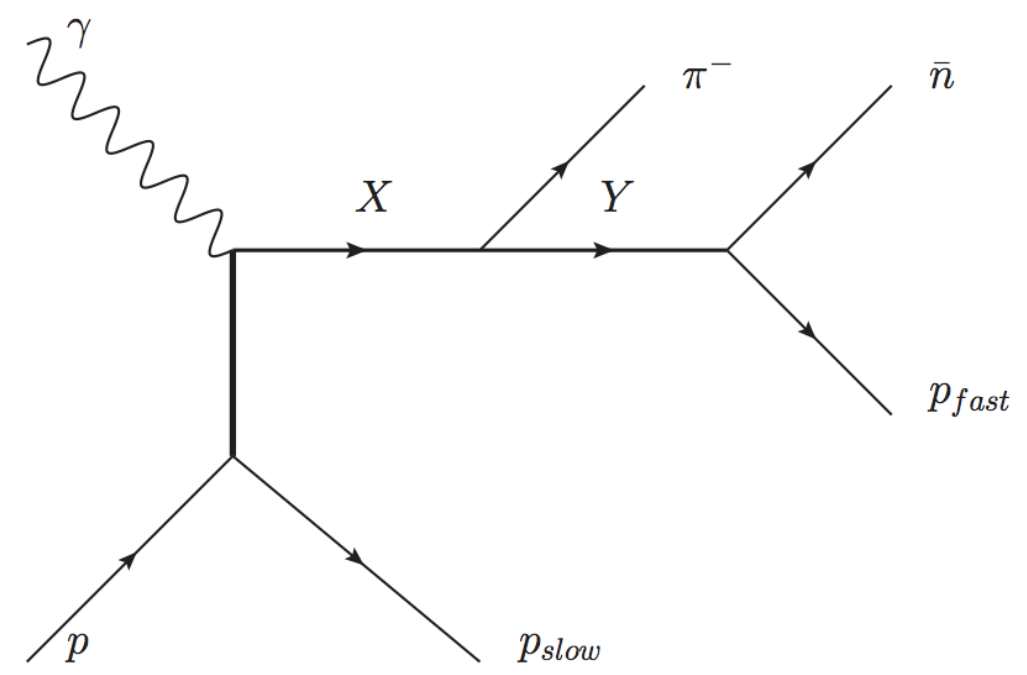

Figure 5.1: Production model used for simulation. 


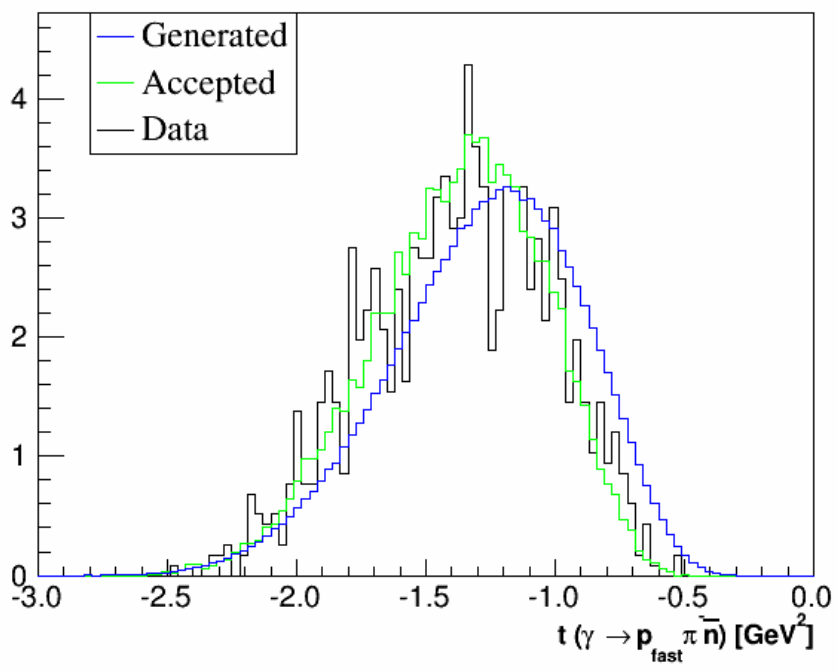

Figure 5.2: Comparison of MC and data.

\subsection{Simulation and Acceptance Calculation}

The simulation model used was based on the diagram shown in Figure 5.1. The input parameters to the event generator GENR8 were tuned to match the data as shown in Fig. 5.2. The mass distributions were measured from the data and used as an input to the MC. After the initial input the mass distributions were adjusted to take into account the differences between the experimental data mass distributions and the distributions after detector acceptance was taken into account.

\subsection{Total Cross Section Measurement}

The total cross section was calculated for the $\gamma p \rightarrow p p \bar{n} \pi^{-}$reaction. The cross section was calculated in a straightforward way using Equation 4.1 and by extracting the yield from a fit to the missing neutron similar to Figure 3.3.1. The main difference being the simulation for this reaction is much more refined with 
the assumption that the reaction can be described with the diagram in Figure 5.1. The simulation matches the data well, as shown in Figure 5.2. This resulting total cross section measurement is the first measured in photoproduction (Fig. 5.3). This cross section is roughly twenty times less that what is seen in the same region for the $p \bar{p}$ production.

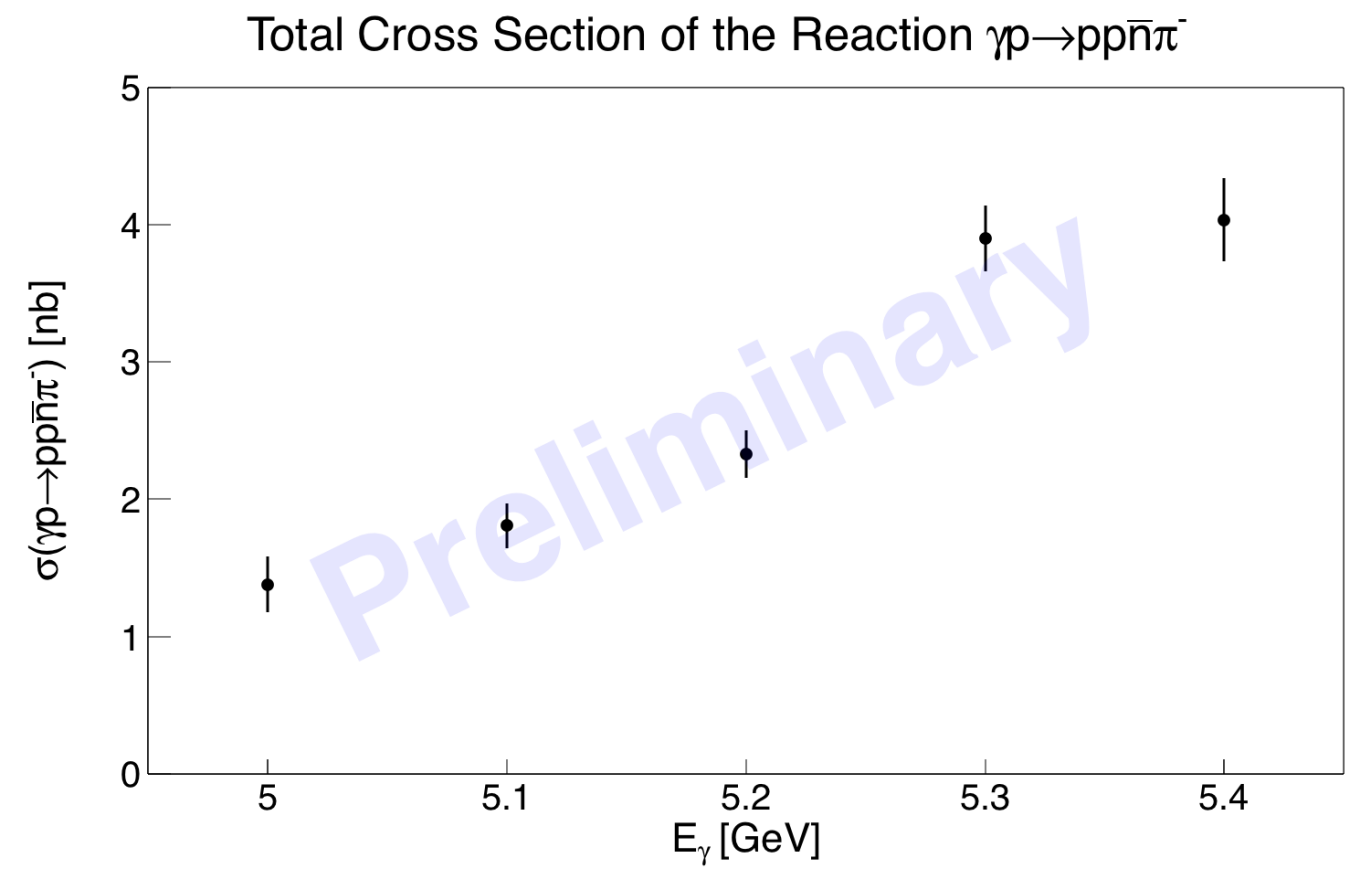

Figure 5.3: Total cross section for the reaction $\gamma p \rightarrow p p \bar{n} \pi^{-}$. 


\section{CHAPTER 6}

\section{Discussion and Conclusions}

Two reactions, $\gamma p \rightarrow p p \bar{p}$ and $\gamma p \rightarrow p p \bar{n} \pi^{-}$have been investigated with data

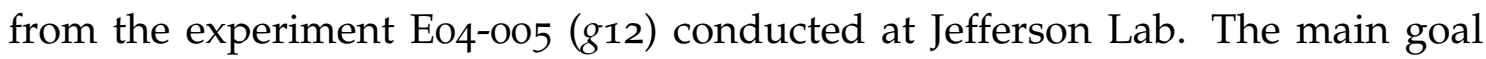
of this work was to investigate the production mechanism of $\gamma p \rightarrow p p \bar{p}$. The g12 dataset provided the world's largest dataset for the reaction $\gamma p \rightarrow p p \bar{p}$, with a total of around 1/4 million events after event selection and about $30 \%$ background.

Previously experiments using various beams and reactions had reported evidence for narrow resonances in the $p \bar{p}$ mass distributions that were conjectured to be baryonium candidates[6][8]. These experiments showed mass distributions with narrow enhancements in the $p \bar{p}$ resonances at 2.02 and $2.02 \mathrm{GeV} / c^{2}$ in this analysis. Evidence for these narrow resonances was not observed (Figs. 3.17, 3.18). Further work could be done in order to set an upper limit for the proposed narrow resonances using the Feldman-Cousins method, which could easily done once the results are published [30].

In particular, the total cross section for $\gamma p \rightarrow p p \bar{p}$ has been measured by two previous experiments covering the $E_{\gamma}$ range from 3.95 to $6.40 \mathrm{GeV}$ [6][8]. Other higher statistics experiments from CLAS were never published. These reactions 
suffered from a lack of statistics and Monte Carlo models that did not accurately represent the reaction.

In this analysis, the total cross section was measured for the reaction $\gamma p \rightarrow$ $p p \bar{p}$. The total cross sections starts at $1.3 \mathrm{nb}$ and rises to $63.3 \mathrm{nb}$ over the photon energy range of 3.95 to $5.45 \mathrm{GeV}$. Some of the LAMP2 data points disagree by a factor of 2 in the middle of the photon energy range and the DESY measurements are roughly a factor of two higher. These experiments suffered from poor statistics, both with approximately 200 events which were measured using simulation models that could not have been investigated with great detail due to the low statistics. CLAS has two unpublished measurements in roughly the same beam energy range. The first measurement was made using 2,500 events and is roughly $30 \%$ higher than this measurement but agrees near threshold [13]. The most recent unpublished CLAS results are much closer agreement than all other results, it was the next highest statistics experiment with 25,000 events [11].

As with previous experiments studying the reaction $\gamma p \rightarrow p p \bar{p}$, one of the main issues in measuring the cross sections is determining the detector acceptance accurately. Three body phase space and meson exchange Monte Carlo models did not accurately represent the data. The solution to this problem was to write an event weighting program that uses $\frac{d^{2} \sigma}{d M(p p) d \cos \left(\theta_{\bar{p}}^{*}\right)}$ and one dimensional photon flux normalized profiles: $t_{\text {baryon }}, M\left(p_{\text {fast }} \bar{p}\right)$, and $\cos \left(\theta^{*}\right)$. The model is ad hoc and utilizes a feedback loop using the cross sections and flux normalized yields to generate the events that are then used as the input model for the next iteration of simulation. For the first time the simulation matches the data well in virtually all kinematic distributions that were thought to be an important factor in the acceptance such as particle momenta, angular distributions, mass distributions, etc. This allows for more realistic cross section 
measurements. The total cross section is then determined using the integral of a Legendre polynomial fit to the differential cross section $d \sigma / d \cos \left(\theta_{\bar{p}}^{C M}\right)$ which allows another safety factor in removing model dependence in the total cross section.

Overall, three sets of differential cross sections were measured for the first time: $d \sigma / d \cos \left(\theta_{\bar{p}}^{C M}\right), d \sigma / d M(p p)$, and $d \sigma / d M(p \bar{p})$. These cross sections provide information about the production. The differential cross section for the angular distributions shows a mainly uniform distribution with a more forward going component. The uniform distribution with the increase as $\cos \left(\theta_{\bar{p}}^{C M}\right)$ approaches 1.0 is evidence that $p \bar{p}$ is really from a combination of production mechanisms with meson exchange models probably being favored. The mass distribution cross sections have been used to constrain the simulation model. The mass distributions also show disagreement with three-body phase space.

There are many future prospects for studying $\gamma p \rightarrow p p \bar{p}$ with not only this dataset, being the first of many high statistics runs, but also with more to come from new experimental apparatuses at Jefferson Lab: CLAS12 and GlueX. Studying the production mechanism through interpreting the cross section using theoretical models is already in progress with plans to expand the models used [14]. Measuring beam helicity asymmetry has already been shown to be sensitive to resonances and may prove to be useful in uncovering how $p \bar{p}$ pairs are produced [31, 32]. Finally, a future partial wave analysis could help the understanding of the production mechanism by extracting production amplitudes for various intermediate states [33]. 


\section{BIBLIOGRAPHY}

[1] Wikipedia, the free encyclopedia, "The standard model of elementary particles," 2017.

[2] M. Gell-Mann, THE EIGHTFOLD WAY: A THEORY OF STRONG INTERACTION SYMMETRY. Mar 1961.

[3] C. D. Anderson, "The positive electron," Phys. Rev., vol. 43, pp. 491-494, Mar 1933.

[4] P. Dirac, "Theory of electrons and positrons," 12 1933. Nobel Lecture by Paul Dirac.

[5] O. Chamberlain, E. Segrè, C. Wiegand, and T. Ypsilantis, "Observation of antiprotons," Phys. Rev., vol. 100, pp. 947-950, Nov 1955.

[6] P. Benkheiri et al., "Evidence for Two Narrow p anti-p Resonances at 2020$\mathrm{MeV}$ and 2200-MeV," Phys. Lett., vol. B68, p. 483, 1977.

[7] B. G. Gibbard et al., "CROSS-SECTIONS AND POSSIBLE RESONANCES IN P ANTI-P ELECTROPRODUCTION," Phys. Rev. Lett., vol. 42, pp. 15931596, 1979 .

[8] J. Bodenkamp, D. C. Fries, A. Markou, E. Seitz, H. J. Behrend, W. P. Hesse, T. Miyachi, W. A. McNeely, Jr., and V. Schroder, “MEASUREMENT OF THE 
REACTION gamma $\mathrm{p} \longrightarrow \mathrm{p}$ anti-p p AT PHOTON ENERGIES $4.7-\mathrm{GeV}<=$ E (gamma) <=6.6-GeV," Nucl. Phys., vol. B255, p. 717, 1985.

[9] A. Ferrer, A. A. Grigorian, V. F. Perepelitsa, and P. Sonderegger, "Comprehensive evidence about a narrow $\mathrm{p}$ anti-p state of mass $2.02-\mathrm{GeV} / \mathrm{c}^{* *} 2$," Eur. Phys. J., vol. C10, pp. 249-263, 1999.

[10] M. N. Oakden and M. R. Pennington, "Amplitude analysis of data on anti-p p $\rightarrow$ pi pi at low-energy," Nucl. Phys., vol. A574, pp. 731-754, 1994.

[11] B. Stokes, Search for Resonances in the Photoproduction of Proton-Antiproton Pairs. PhD thesis, Florida State U., 2006.

[12] K. A. Olive et al., "Review of Particle Physics," Chin. Phys., vol. C38, p. 090001, 2014.

[13] M. R. V. Koubarovski, M. Battaglieri, "Measurement of the Reaction gamma $p \rightarrow p$ pbar p at Photon Energies from Threshold up to $5.23 \mathrm{GeV}$ and Search for Resonances in the ppbar System with CLAS," CLAS Note, vol. 106, pp. 137,2003 .

[14] T. Gutsche, S. Kuleshov, V. E. Lyubovitskij, and I. T. Obukhovsky, "Search for the glueball content of hadrons in $\gamma p$ interactions at GlueX," Phys. Rev., vol. D94, no. 3, p. 034010, 2016.

[15] P. Eugenio, C. Salgado, D. Weygand, et al., "Search for new forms of hadronic matter in photoproduction," 122003.

[16] D. Sober, H. Crannell, A. Longhi, S. Matthews, J. O’Brien, B. Berman, W. Briscoe, P. L. Cole, J. Connelly, W. Dodge, L. Murphy, S. Philips, M. Dugger, D. Lawrence, B. Ritchie, E. Smith, J. M. Lambert, E. Anciant, G. Audit, 
T. Auger, C. Marchand, M. Klusman, J. Napolitano, M. Khandaker, C. Salgado, and A. Sarty, "The bremsstrahlung tagged photon beam in hall $\mathrm{b}$ at jlab," Nuclear Instruments and Methods in Physics Research Section A: Accelerators, Spectrometers, Detectors and Associated Equipment, vol. 440, no. 2, pp. 263 $-284,2000$.

[17] Y. G. Sharabian et al., "A new highly segmented start counter for the CLAS detector," Nucl. Instrum. Meth., vol. A556, pp. 246-258, 2006.

[18] M. Mestayer, D. Carman, B. Asavapibhop, F. Barbosa, P. Bonneau, S. Christo, G. Dodge, T. Dooling, W. Duncan, S. Dytman, R. Feuerbach, G. Gilfoyle, V. Gyurjyan, K. Hicks, R. Hicks, C. Hyde-Wright, G. Jacobs, A. Klein, F. Klein, M. Kossov, S. Kuhn, R. Magahiz, R. Major, C. Martin, T. McGuckin, J. McNabb, R. Miskimen, J. Mueller, B. Niczyporuk, J. O'Meara, L. Qin, B. Raue, J. Robb, F. Roudot, R. Schumacher, D. Tedeschi, R. Thompson, D. Tilles, W. Tuzel, K. VanSyoc, M. Vineyard, L. Weinstein, G. Wilkin, A. Yegneswaran, and J. Yun, "The clas drift chamber system," Nuclear Instruments and Methods in Physics Research Section A: Accelerators, Spectrometers, Detectors and Associated Equipment, vol. 449, no. 1, pp. 81 $111,2000$.

[19] E. Smith, T. Carstens, J. Distelbrink, M. Eckhause, H. Egiyan, L. Elouadrhiri, J. Ficenec, M. Guidal, A. Hancock, F. Hersman, M. Holtrop, D. Jenkins, W. Kim, K. Loukachine, K. MacArthur, C. Marchand, B. Mecking, G. Mutchler, D. Schutt, L. Smith, T. Smith, S. Taylor, T. Tung, A. Weisenberger, and R. Welsh, "The time-of-flight system for clas," Nuclear Instruments and Methods in Physics Research Section A: Accelerators, Spectrometers, Detectors and Associated Equipment, vol. 432, no. 2, pp. 265-298, 1999. 
[20] G. Adams, V. Burkert, R. Carl, T. Carstens, V. Frolov, L. Houghtlin, G. Jacobs, M. Kossov, M. Klusman, B. Kross, M. Onuk, J. Napolitano, J. Price, C. Riggs, Y. Sharabian, A. Stavinsky, L. Smith, W. Stephens, P. Stoler, W. Tuzel, K. Ullrich, A. Vlassov, A. Weisenberger, M. Witkowski, B. Wojtekhowski, P. Yergin, and C. Zorn, "The clas cherenkov detector," Nuclear Instruments and Methods in Physics Research Section A: Accelerators, Spectrometers, Detectors and Associated Equipment, vol. 465, no. 2, pp. 414-427, 2001.

[21] M. Amarian, G. Asryan, K. Beard, W. Brooks, V. Burkert, T. Carstens, A. Coleman, R. Demirchyan, Y. Efremenko, H. Egiyan, K. Egiyan, H. Funsten, V. Gavrilov, K. Giovanetti, R. Marshall, B. Mecking, R. Minehart, H. Mkrtchan, M. Ohandjanyan, Y. Sharabian, L. Smith, S. Stepanyan, W. Stephens, T. Tung, and C. Zorn, "The clas forward electromagnetic calorimeter," Nuclear Instruments and Methods in Physics Research Section A: Accelerators, Spectrometers, Detectors and Associated Equipment, vol. 46o, no. 2, pp. $239-265,2001$.

[22] B. A. Mecking et al., "The CEBAF Large Acceptance Spectrometer (CLAS)," Nucl. Instrum. Meth., vol. A503, pp. 513-553, 2003.

[23] J. G. et al., "g12 Analysis Procedures, Statistics and Systematics," CLAS Note, vol. 101, pp. 1-124, 2017.

[24] E. Pasyuk, "Energy loss corrections for charged particles in CLAS," CLASNote, vol. 016, 2007.

[25] D. Lersch. personal communication.

[26] D. Keller, "Techniques in Kinematic Fitting," CLAS Note, vol. 101, pp. 1-72, 2010. 
[27] D. P. Barber et al., "Elastic Anti-proton - Proton Photoproduction Between Threshold and 4.8-GeV," Phys. Lett., vol. B9o, p. 470, 1980.

[28] V. Lyubovitskij. personal communication.

[29] M. C. Kunkel, "Measurement of Cross-Sections of exclusive $\pi^{0}$ Photoproduction on Hydrogen from 1.1 GeV $-5.45 \mathrm{GeV}$ using $e^{+} e^{-} \gamma$ decay from the CLAS/g12 Data," CLAS Note, vol. 105, 2017.

[30] G. J. Feldman and R. D. Cousins, "A Unified approach to the classical statistical analysis of small signals," Phys. Rev., vol. D57, pp. 3873-3889, 1998.

[31] S. Strauch et al., "Beam-helicity asymmetries in double-charged-pion photoproduction on the proton," Phys. Rev. Lett., vol. 95, p. 162003, 2005.

[32] R. Badui, The Beam-Helicity Asymmetry for Two Pseudoscalar Mesons in Photoproduction and a Partial Wave Analysis for Excited Hyperons. PhD thesis, Florida International U., 2016.

[33] C. W. Salgado and D. P. Weygand, "On the Partial-Wave Analysis of Mesonic Resonances Decaying to Multiparticle Final States Produced by Polarized Photons," Phys. Rept., vol. 537, pp. 1-58, 2014. 


\section{Appendix A}

\section{Appendices}

\section{A.1 Tabular Data: Total Cross Section for $\gamma p \rightarrow p p \bar{p}$}

\begin{tabular}{ccc}
$E_{\gamma}[\mathrm{GeV}]$ & $\sigma[n b]$ & $\sigma_{\text {error }}[n b]$ \\
\hline 3.975 & 1.31792 & 0.469841 \\
4.025 & 1.3873 & 0.389005 \\
4.075 & 3.9425 & 0.770502 \\
4.125 & 4.55707 & 0.744299 \\
4.175 & 7.02158 & 0.915624 \\
4.225 & 8.09187 & 0.970549 \\
4.275 & 10.4645 & 1.10503 \\
4.325 & 13.8297 & 1.39859 \\
4.375 & 16.04 & 1.60759 \\
4.425 & 19.3515 & 2.04504 \\
4.475 & 20.0685 & 1.53721 \\
4.525 & 23.3651 & 1.65693 \\
4.575 & 26.8512 & 1.95527 \\
4.625 & 27.9179 & 1.97359 \\
4.675 & 30.8073 & 2.08578 \\
4.725 & 31.7473 & 2.11193 \\
\hline & &
\end{tabular}


Table A.1 - Continued

\begin{tabular}{ccc}
$E_{\gamma}[\mathrm{GeV}]$ & $\sigma[n b]$ & $\sigma_{\text {error }}[n b]$ \\
\hline 4.775 & 38.8543 & 2.83257 \\
4.825 & 42.8545 & 2.73401 \\
4.875 & 40.8044 & 2.45703 \\
4.925 & 40.2116 & 2.33538 \\
4.975 & 46.7572 & 2.98084 \\
5.025 & 52.0399 & 3.24542 \\
5.075 & 47.7458 & 2.68348 \\
5.125 & 53.8525 & 3.04142 \\
5.175 & 55.5306 & 3.24479 \\
5.225 & 56.6883 & 3.30768 \\
5.275 & 57.8514 & 3.84199 \\
5.325 & 58.168 & 3.27588 \\
5.375 & 67.2488 & 3.7913 \\
5.425 & 62.3424 & 3.46445 \\
\hline
\end{tabular}

A.2 Tabular Data: $d \sigma / d \cos \theta_{\bar{p}}^{\text {C.M. }}$ for $\gamma p \rightarrow p p \bar{p}$

\begin{tabular}{cccccc}
$E_{\text {beam }}^{\text {lab }}[\mathrm{GeV}]$ & $\pm d E_{\text {beam }}^{\text {lab }}$ & $\cos \theta_{\overline{\bar{p}}}^{\mathrm{C} . M .}$ & $\pm d \cos \theta_{\overline{\bar{p}}}^{\mathrm{C} . M .}$ & $\frac{d \sigma}{d \Omega}[n b]$ & $\sigma^{\text {stat }}$ \\
\hline 3.95 & 0.05 & -0.90 & 0.20 & 0.3051 & 0.091803 \\
3.95 & 0.05 & -0.70 & 0.20 & 0.4485 & 0.134948 \\
3.95 & 0.05 & -0.50 & 0.20 & 0.3819 & 0.127585 \\
3.95 & 0.05 & -0.30 & 0.20 & 0.4144 & 0.138441 \\
3.95 & 0.05 & -0.10 & 0.20 & 0.5011 & 0.167423 \\
3.95 & 0.05 & 0.10 & 0.20 & 0.3866 & 0.116315 \\
\hline
\end{tabular}


Table A.2 - Continued

\begin{tabular}{|c|c|c|c|c|c|}
\hline$E_{\text {beam }}^{\text {lab }}[\mathrm{GeV}]$ & $\pm d E_{\text {beam }}^{\text {lab }}$ & $\cos \theta_{\bar{p}}^{C . M .}$ & $\pm d \cos \theta_{\bar{p}}^{C . M .}$ & $\frac{d \sigma}{d \Omega}[n b]$ & $\sigma^{\text {stat }}$ \\
\hline 3.95 & 0.05 & 0.30 & 0.20 & 0.9456 & 0.173227 \\
\hline 3.95 & 0.05 & 0.50 & 0.20 & 1.0230 & 0.166607 \\
\hline 3.95 & 0.05 & 0.70 & 0.20 & 1.0689 & 0.185835 \\
\hline 3.95 & 0.05 & 0.90 & 0.20 & 1.1145 & 0.157505 \\
\hline 4.00 & 0.05 & -0.90 & 0.20 & 0.5626 & 0.103256 \\
\hline 4.00 & 0.05 & -0.70 & 0.20 & 0.4102 & 0.103049 \\
\hline 4.00 & 0.05 & -0.50 & 0.20 & 0.3879 & 0.097460 \\
\hline 4.00 & 0.05 & -0.30 & 0.20 & 0.4474 & 0.112395 \\
\hline 4.00 & 0.05 & -0.10 & 0.20 & 0.6792 & 0.136892 \\
\hline 4.00 & 0.05 & 0.10 & 0.20 & 0.8391 & 0.137668 \\
\hline 4.00 & 0.05 & 0.30 & 0.20 & 0.8716 & 0.142986 \\
\hline 4.00 & 0.05 & 0.50 & 0.20 & 0.5805 & 0.104658 \\
\hline 4.00 & 0.05 & 0.70 & 0.20 & 1.0607 & $0.14795^{2}$ \\
\hline 4.00 & 0.05 & 0.90 & 0.20 & 1.0973 & 0.130125 \\
\hline 4.05 & 0.05 & -0.90 & 0.20 & 1.3355 & 0.198058 \\
\hline 4.05 & 0.05 & -0.70 & 0.20 & 1.2471 & 0.191302 \\
\hline 4.05 & 0.05 & -0.50 & 0.20 & 1.3250 & 0.209438 \\
\hline 4.05 & 0.05 & -0.30 & 0.20 & 1.8222 & 0.263945 \\
\hline 4.05 & 0.05 & -0.10 & 0.20 & 1.9929 & 0.267491 \\
\hline 4.05 & 0.05 & 0.10 & 0.20 & 2.0768 & 0.243441 \\
\hline 4.05 & 0.05 & 0.30 & 0.20 & 2.1555 & 0.262477 \\
\hline 4.05 & 0.05 & 0.50 & 0.20 & 2.4495 & $0.26295^{1}$ \\
\hline 4.05 & 0.05 & 0.70 & 0.20 & 2.7578 & 0.280868 \\
\hline 4.05 & 0.05 & 0.90 & 0.20 & 2.5501 & 0.237865 \\
\hline
\end{tabular}


Table A.2 - Continued

\begin{tabular}{|c|c|c|c|c|c|}
\hline$E_{\text {beam }}^{\text {lab }}[\mathrm{GeV}]$ & $\pm d E_{\text {beam }}^{\text {lab }}$ & $\cos \theta_{\bar{p}}^{C . M .}$ & $\pm d \cos \theta_{\bar{p}}^{C . M}$ & $\frac{d \sigma}{d \Omega}[n b]$ & $\sigma^{\text {stat }}$ \\
\hline 4.10 & 0.05 & -0.90 & 0.20 & 1.4419 & 0.190291 \\
\hline 4.10 & 0.05 & -0.70 & 0.20 & 1.5237 & 0.220908 \\
\hline 4.10 & 0.05 & -0.50 & 0.20 & 2.0707 & 0.235678 \\
\hline 4.10 & 0.05 & -0.30 & 0.20 & 1.9653 & 0.223701 \\
\hline 4.10 & 0.05 & -0.10 & 0.20 & 1.9858 & 0.238706 \\
\hline 4.10 & 0.05 & 0.10 & 0.20 & 2.1481 & 0.236193 \\
\hline 4.10 & 0.05 & 0.30 & 0.20 & 2.3513 & 0.237217 \\
\hline 4.10 & 0.05 & 0.50 & 0.20 & 2.5225 & 0.237624 \\
\hline 4.10 & 0.05 & 0.70 & 0.20 & $3 \cdot 5198$ & 0.285973 \\
\hline 4.10 & 0.05 & 0.90 & 0.20 & 3.2562 & 0.236852 \\
\hline 4.15 & 0.05 & -0.90 & 0.20 & 2.4579 & 0.237271 \\
\hline 4.15 & 0.05 & -0.70 & 0.20 & 2.7119 & 0.281236 \\
\hline 4.15 & 0.05 & -0.50 & 0.20 & 3.0384 & 0.296178 \\
\hline 4.15 & 0.05 & -0.30 & 0.20 & 3.2631 & 0.282072 \\
\hline 4.15 & 0.05 & -0.10 & 0.20 & 3.2664 & 0.303913 \\
\hline 4.15 & 0.05 & 0.10 & 0.20 & 3.0311 & 0.269637 \\
\hline 4.15 & 0.05 & 0.30 & 0.20 & $3 \cdot 3162$ & 0.271443 \\
\hline 4.15 & 0.05 & 0.50 & 0.20 & $4 \cdot 3192$ & 0.315355 \\
\hline 4.15 & 0.05 & 0.70 & 0.20 & $4 \cdot 5695$ & 0.318092 \\
\hline 4.15 & 0.05 & 0.90 & 0.20 & 5.1341 & 0.310338 \\
\hline 4.20 & 0.05 & -0.90 & 0.20 & 2.7221 & 0.253661 \\
\hline 4.20 & 0.05 & -0.70 & 0.20 & 2.9802 & 0.292555 \\
\hline 4.20 & 0.05 & -0.50 & 0.20 & $3 \cdot 7928$ & 0.333367 \\
\hline 4.20 & 0.05 & -0.30 & 0.20 & 3.2124 & 0.278408 \\
\hline
\end{tabular}


Table A.2 - Continued

\begin{tabular}{|c|c|c|c|c|c|}
\hline$E_{\text {beam }}^{\text {lab }}[\mathrm{GeV}]$ & $\pm d E_{\text {beam }}^{\text {lab }}$ & $\cos \theta_{\bar{p}}^{\text {C.M. }}$ & $\pm d \cos \theta_{\bar{p}}^{C} \cdot M$ & $\frac{d \sigma}{d \Omega}[n b]$ & $\sigma^{\text {stat }}$ \\
\hline 4.20 & 0.05 & -0.10 & 0.20 & 3.6889 & 0.313677 \\
\hline 4.20 & 0.05 & 0.10 & 0.20 & 3.4901 & 0.277804 \\
\hline 4.20 & 0.05 & 0.30 & 0.20 & 4.4338 & 0.313836 \\
\hline 4.20 & 0.05 & 0.50 & 0.20 & 4.7490 & 0.316450 \\
\hline 4.20 & 0.05 & 0.70 & 0.20 & 5.1608 & 0.332705 \\
\hline 4.20 & 0.05 & 0.90 & 0.20 & 6.2293 & 0.344229 \\
\hline 4.25 & 0.05 & -0.90 & 0.20 & 3.7167 & 0.302150 \\
\hline 4.25 & 0.05 & -0.70 & 0.20 & $4 \cdot 3114$ & 0.336507 \\
\hline 4.25 & 0.05 & -0.50 & 0.20 & 5.6134 & 0.417611 \\
\hline 4.25 & 0.05 & -0.30 & 0.20 & $4 \cdot 5237$ & 0.341365 \\
\hline 4.25 & 0.05 & -0.10 & 0.20 & $5 \cdot 3184$ & 0.364532 \\
\hline 4.25 & 0.05 & 0.10 & 0.20 & $4 \cdot 7700$ & 0.320444 \\
\hline 4.25 & 0.05 & 0.30 & 0.20 & 5.2148 & 0.344030 \\
\hline 4.25 & 0.05 & 0.50 & 0.20 & 5.1560 & $0.32595^{8}$ \\
\hline 4.25 & 0.05 & 0.70 & 0.20 & 6.5940 & 0.371376 \\
\hline 4.25 & 0.05 & 0.90 & 0.20 & 7.1040 & 0.357116 \\
\hline $4 \cdot 30$ & 0.05 & -0.90 & 0.20 & 4.7198 & 0.376894 \\
\hline $4 \cdot 30$ & 0.05 & -0.70 & 0.20 & 5.1105 & 0.403009 \\
\hline 4.30 & 0.05 & -0.50 & 0.20 & 6.5280 & 0.482639 \\
\hline $4 \cdot 30$ & 0.05 & -0.30 & 0.20 & $7 \cdot 3560$ & $0.48355^{8}$ \\
\hline $4 \cdot 30$ & 0.05 & -0.10 & 0.20 & 6.3893 & 0.433448 \\
\hline $4 \cdot 30$ & 0.05 & 0.10 & 0.20 & 6.3403 & 0.427183 \\
\hline $4 \cdot 30$ & 0.05 & 0.30 & 0.20 & 6.7323 & 0.426173 \\
\hline $4 \cdot 30$ & 0.05 & 0.50 & 0.20 & 7.0781 & 0.421786 \\
\hline
\end{tabular}


Table A.2 - Continued

\begin{tabular}{|c|c|c|c|c|c|}
\hline$E_{\text {beam }}^{\text {lab }}[\mathrm{GeV}]$ & $\pm d E_{\text {beam }}^{l a b}$ & $\cos \theta_{\bar{p}}^{\text {C.M. }}$ & $\pm d \cos \theta_{\bar{p}}^{C} \cdot M$ & $\frac{d \sigma}{d \Omega}[n b]$ & $\sigma^{\text {stat }}$ \\
\hline 4.30 & 0.05 & 0.70 & 0.20 & 8.8793 & 0.476284 \\
\hline $4 \cdot 30$ & 0.05 & 0.90 & 0.20 & 10.0151 & 0.477594 \\
\hline 4.35 & 0.05 & -0.90 & 0.20 & 5.2803 & 0.428330 \\
\hline $4 \cdot 35$ & 0.05 & -0.70 & 0.20 & 6.2113 & 0.496079 \\
\hline $4 \cdot 35$ & 0.05 & -0.50 & 0.20 & $7 \cdot 4272$ & 0.540998 \\
\hline $4 \cdot 35$ & 0.05 & -0.30 & 0.20 & $7 \cdot 45^{25}$ & 0.520400 \\
\hline $4 \cdot 35$ & 0.05 & -0.10 & 0.20 & 7.8430 & 0.523845 \\
\hline $4 \cdot 35$ & 0.05 & 0.10 & 0.20 & 7.9961 & 0.506241 \\
\hline $4 \cdot 35$ & 0.05 & 0.30 & 0.20 & 8.2311 & 0.488275 \\
\hline $4 \cdot 35$ & 0.05 & 0.50 & 0.20 & 9.0005 & 0.501561 \\
\hline $4 \cdot 35$ & 0.05 & 0.70 & 0.20 & 9.9903 & 0.541543 \\
\hline $4 \cdot 35$ & 0.05 & 0.90 & 0.20 & 10.7679 & 0.526645 \\
\hline 4.40 & 0.05 & -0.90 & 0.20 & 6.7558 & 0.555808 \\
\hline 4.40 & 0.05 & -0.70 & 0.20 & 7.9240 & 0.655721 \\
\hline 4.40 & 0.05 & -0.50 & 0.20 & 9.2655 & 0.686317 \\
\hline 4.40 & 0.05 & -0.30 & 0.20 & 9.1416 & 0.664920 \\
\hline 4.40 & 0.05 & -0.10 & 0.20 & 9.6449 & 0.654144 \\
\hline 4.40 & 0.05 & 0.10 & 0.20 & 8.0151 & 0.570959 \\
\hline 4.40 & 0.05 & 0.30 & 0.20 & 9.6255 & 0.641112 \\
\hline 4.40 & 0.05 & 0.50 & 0.20 & 11.4807 & 0.671703 \\
\hline 4.40 & 0.05 & 0.70 & 0.20 & 12.2798 & 0.683082 \\
\hline 4.40 & 0.05 & 0.90 & 0.20 & 12.6244 & 0.668921 \\
\hline 4.45 & 0.05 & -0.90 & 0.20 & 7.6009 & 0.445693 \\
\hline 4.45 & 0.05 & -0.70 & 0.20 & 7.6924 & 0.462209 \\
\hline
\end{tabular}


Table A.2 - Continued

\begin{tabular}{|c|c|c|c|c|c|}
\hline$E_{\text {beam }}^{\text {lab }}[\mathrm{GeV}]$ & $\pm d E_{\text {beam }}^{l a b}$ & $\cos \theta_{\bar{p}}^{\text {C.M. }}$ & $\pm d \cos \theta_{\bar{p}}^{C} \cdot M$ & $\frac{d \sigma}{d \Omega}[n b]$ & $\sigma^{\text {stat }}$ \\
\hline 4.45 & 0.05 & -0.50 & 0.20 & $9 \cdot 5758$ & 0.508740 \\
\hline $4 \cdot 45$ & 0.05 & -0.30 & 0.20 & 8.5916 & 0.469206 \\
\hline $4 \cdot 45$ & 0.05 & -0.10 & 0.20 & 9.7406 & 0.488123 \\
\hline $4 \cdot 45$ & 0.05 & 0.10 & 0.20 & 9.8337 & 0.472295 \\
\hline 4.45 & 0.05 & 0.30 & 0.20 & 10.3212 & 0.486421 \\
\hline 4.45 & 0.05 & 0.50 & 0.20 & 10.8498 & 0.484377 \\
\hline 4.45 & 0.05 & 0.70 & 0.20 & 12.8532 & 0.526180 \\
\hline 4.45 & 0.05 & 0.90 & 0.20 & 13.2831 & 0.512185 \\
\hline 4.50 & 0.05 & -0.90 & 0.20 & 8.7426 & 0.471415 \\
\hline 4.50 & 0.05 & -0.70 & 0.20 & 10.0999 & 0.527496 \\
\hline 4.50 & 0.05 & -0.50 & 0.20 & 10.8845 & 0.544337 \\
\hline 4.50 & 0.05 & -0.30 & 0.20 & 11.0942 & 0.528576 \\
\hline 4.50 & 0.05 & -0.10 & 0.20 & 11.0512 & 0.515041 \\
\hline 4.50 & 0.05 & 0.10 & 0.20 & 10.6976 & 0.491269 \\
\hline 4.50 & 0.05 & 0.30 & 0.20 & 11.9202 & 0.520370 \\
\hline 4.50 & 0.05 & 0.50 & 0.20 & 12.5064 & 0.513730 \\
\hline 4.50 & 0.05 & 0.70 & 0.20 & 14.1449 & 0.554411 \\
\hline 4.50 & 0.05 & 0.90 & 0.20 & 15.6840 & 0.566186 \\
\hline $4 \cdot 55$ & 0.05 & -0.90 & 0.20 & 10.5635 & 0.584514 \\
\hline $4 \cdot 55$ & 0.05 & -0.70 & 0.20 & 11.0109 & 0.610463 \\
\hline 4.55 & 0.05 & -0.50 & 0.20 & 11.8700 & 0.610480 \\
\hline 4.55 & 0.05 & -0.30 & 0.20 & 11.7749 & 0.592983 \\
\hline 4.55 & 0.05 & -0.10 & 0.20 & 12.0303 & 0.585904 \\
\hline 4.55 & 0.05 & 0.10 & 0.20 & 13.7437 & 0.623756 \\
\hline
\end{tabular}


Table A.2 - Continued

\begin{tabular}{|c|c|c|c|c|c|}
\hline$E_{\text {beam }}^{\text {lab }}[\mathrm{GeV}]$ & $\pm d E_{\text {beam }}^{\text {lab }}$ & $\cos \theta_{\bar{p}}^{C . M}$ & $\pm d \cos \theta_{\bar{p}}^{C . M .}$ & $\frac{d \sigma}{d \Omega}[n b]$ & $\sigma^{\text {stat }}$ \\
\hline 4.55 & 0.05 & 0.30 & 0.20 & 14.9307 & 0.640423 \\
\hline $4 \cdot 55$ & 0.05 & 0.50 & 0.20 & 14.6519 & 0.616106 \\
\hline $4 \cdot 55$ & 0.05 & 0.70 & 0.20 & 16.2389 & 0.657608 \\
\hline $4 \cdot 55$ & 0.05 & 0.90 & 0.20 & $17 \cdot 4411$ & 0.655691 \\
\hline 4.60 & 0.05 & -0.90 & 0.20 & 11.0404 & 0.577966 \\
\hline 4.60 & 0.05 & -0.70 & 0.20 & 12.6109 & 0.648413 \\
\hline 4.60 & 0.05 & -0.50 & 0.20 & 13.0215 & 0.649594 \\
\hline 4.60 & 0.05 & -0.30 & 0.20 & 12.6809 & 0.613040 \\
\hline 4.60 & 0.05 & -0.10 & 0.20 & 11.9542 & 0.578023 \\
\hline 4.60 & 0.05 & 0.10 & 0.20 & 12.8706 & 0.580359 \\
\hline 4.60 & 0.05 & 0.30 & 0.20 & 14.9603 & 0.629683 \\
\hline 4.60 & 0.05 & 0.50 & 0.20 & 15.6589 & 0.635089 \\
\hline 4.60 & 0.05 & 0.70 & 0.20 & 16.9703 & 0.662504 \\
\hline 4.60 & 0.05 & 0.90 & 0.20 & 17.8214 & 0.658097 \\
\hline 4.65 & 0.05 & -0.90 & 0.20 & 12.7742 & 0.643292 \\
\hline 4.65 & 0.05 & -0.70 & 0.20 & 12.4542 & 0.636568 \\
\hline 4.65 & 0.05 & -0.50 & 0.20 & 13.6302 & 0.647848 \\
\hline 4.65 & 0.05 & -0.30 & 0.20 & 13.4883 & 0.620490 \\
\hline 4.65 & 0.05 & -0.10 & 0.20 & 13.1088 & 0.601510 \\
\hline 4.65 & 0.05 & 0.10 & 0.20 & 14.4755 & 0.625005 \\
\hline 4.65 & 0.05 & 0.30 & 0.20 & 16.0241 & 0.662904 \\
\hline 4.65 & 0.05 & 0.50 & 0.20 & $17 \cdot 3545$ & 0.672252 \\
\hline 4.65 & 0.05 & 0.70 & 0.20 & 19.3691 & 0.725455 \\
\hline 4.65 & 0.05 & 0.90 & 0.20 & 21.3576 & 0.746084 \\
\hline
\end{tabular}


Table A.2 - Continued

\begin{tabular}{|c|c|c|c|c|c|}
\hline$E_{\text {beam }}^{\text {lab }}[\mathrm{GeV}]$ & $\pm d E_{\text {beam }}^{\text {lab }}$ & $\cos \theta_{\bar{p}}^{C . M}$ & $\pm d \cos \theta_{\bar{p}}^{C . M .}$ & $\frac{d \sigma}{d \Omega}[n b]$ & $\sigma^{\text {stat }}$ \\
\hline $4 \cdot 70$ & 0.05 & -0.90 & 0.20 & 12.5712 & 0.638171 \\
\hline $4 \cdot 70$ & 0.05 & -0.70 & 0.20 & 13.7052 & 0.676459 \\
\hline $4 \cdot 70$ & 0.05 & -0.50 & 0.20 & 12.1062 & 0.603524 \\
\hline $4 \cdot 70$ & 0.05 & -0.30 & 0.20 & 13.6085 & 0.621542 \\
\hline $4 \cdot 70$ & 0.05 & -0.10 & 0.20 & 14.5150 & 0.632374 \\
\hline $4 \cdot 70$ & 0.05 & 0.10 & 0.20 & 15.2928 & 0.635387 \\
\hline $4 \cdot 70$ & 0.05 & 0.30 & 0.20 & $17 \cdot 5923$ & 0.695597 \\
\hline $4 \cdot 70$ & 0.05 & 0.50 & 0.20 & $17 \cdot 4500$ & 0.669089 \\
\hline $4 \cdot 70$ & 0.05 & 0.70 & 0.20 & 19.8983 & 0.732349 \\
\hline $4 \cdot 70$ & 0.05 & 0.90 & 0.20 & 21.9968 & 0.757189 \\
\hline $4 \cdot 75$ & 0.05 & -0.90 & 0.20 & 14.7149 & 0.815335 \\
\hline $4 \cdot 75$ & 0.05 & -0.70 & 0.20 & 16.9356 & 0.910779 \\
\hline $4 \cdot 75$ & 0.05 & -0.50 & 0.20 & 16.4645 & 0.846812 \\
\hline $4 \cdot 75$ & 0.05 & -0.30 & 0.20 & 16.5581 & 0.818891 \\
\hline $4 \cdot 75$ & 0.05 & -0.10 & 0.20 & 17.6859 & 0.841704 \\
\hline $4 \cdot 75$ & 0.05 & 0.10 & 0.20 & 19.6632 & 0.883287 \\
\hline $4 \cdot 75$ & 0.05 & 0.30 & 0.20 & 21.6272 & 0.940430 \\
\hline $4 \cdot 75$ & 0.05 & 0.50 & 0.20 & 21.9171 & 0.931252 \\
\hline $4 \cdot 75$ & 0.05 & 0.70 & 0.20 & 22.1977 & 0.921148 \\
\hline $4 \cdot 75$ & 0.05 & 0.90 & 0.20 & 26.5073 & 1.025990 \\
\hline 4.80 & 0.05 & -0.90 & 0.20 & 17.0160 & 0.803626 \\
\hline 4.80 & 0.05 & -0.70 & 0.20 & 16.9346 & 0.803468 \\
\hline 4.80 & 0.05 & -0.50 & 0.20 & 18.0149 & 0.816266 \\
\hline 4.80 & 0.05 & -0.30 & 0.20 & 18.1093 & 0.798318 \\
\hline
\end{tabular}


Table A.2 - Continued

\begin{tabular}{|c|c|c|c|c|c|}
\hline$E_{\text {beam }}^{\text {lab }}[\mathrm{GeV}]$ & $\pm d E_{\text {beam }}^{\text {lab }}$ & $\cos \theta_{\bar{p}}^{C . M}$ & $\pm d \cos \theta_{\bar{p}}^{C . M .}$ & $\frac{d \sigma}{d \Omega}[n b]$ & $\sigma^{\text {stat }}$ \\
\hline 4.80 & 0.05 & -0.10 & 0.20 & 20.7048 & 0.857109 \\
\hline 4.80 & 0.05 & 0.10 & 0.20 & 20.5530 & 0.822843 \\
\hline 4.80 & 0.05 & 0.30 & 0.20 & 23.1966 & 0.881138 \\
\hline 4.80 & 0.05 & 0.50 & 0.20 & $23 \cdot 3417$ & 0.870125 \\
\hline 4.80 & 0.05 & 0.70 & 0.20 & 27.8788 & 0.990808 \\
\hline 4.80 & 0.05 & 0.90 & 0.20 & 28.5228 & 0.976160 \\
\hline 4.85 & 0.05 & -0.90 & 0.20 & 16.2745 & 0.724860 \\
\hline 4.85 & 0.05 & -0.70 & 0.20 & 18.4749 & 0.803233 \\
\hline 4.85 & 0.05 & -0.50 & 0.20 & 17.5595 & 0.740105 \\
\hline 4.85 & 0.05 & -0.30 & 0.20 & 16.5165 & 0.683403 \\
\hline 4.85 & 0.05 & -0.10 & 0.20 & $17 \cdot 3905$ & 0.693677 \\
\hline 4.85 & 0.05 & 0.10 & 0.20 & 19.3789 & 0.735591 \\
\hline 4.85 & 0.05 & 0.30 & 0.20 & 21.5632 & 0.779570 \\
\hline 4.85 & 0.05 & 0.50 & 0.20 & 22.8664 & 0.806812 \\
\hline 4.85 & 0.05 & 0.70 & 0.20 & $25 \cdot 9122$ & 0.870964 \\
\hline 4.85 & 0.05 & 0.90 & 0.20 & 28.0855 & 0.901147 \\
\hline $4 \cdot 90$ & 0.05 & -0.90 & 0.20 & 15.7529 & 0.682381 \\
\hline $4 \cdot 90$ & 0.05 & -0.70 & 0.20 & 16.4283 & 0.716047 \\
\hline 4.90 & 0.05 & -0.50 & 0.20 & $17 \cdot 3858$ & 0.715462 \\
\hline $4 \cdot 90$ & 0.05 & -0.30 & 0.20 & 17.8300 & 0.702902 \\
\hline 4.90 & 0.05 & -0.10 & 0.20 & 18.2466 & 0.697550 \\
\hline $4 \cdot 90$ & 0.05 & 0.10 & 0.20 & 19.2860 & 0.696125 \\
\hline 4.90 & 0.05 & 0.30 & 0.20 & 21.3211 & 0.742641 \\
\hline 4.90 & 0.05 & 0.50 & 0.20 & 22.1310 & 0.744022 \\
\hline
\end{tabular}


Table A.2 - Continued

\begin{tabular}{|c|c|c|c|c|c|}
\hline$E_{\text {beam }}^{l a b}[\mathrm{GeV}]$ & $\pm d E_{\text {beam }}^{\text {lab }}$ & $\cos \theta_{\bar{p}}^{C . M}$ & $\pm d \cos \theta_{\bar{p}}^{C . M .}$ & $\frac{d \sigma}{d \Omega}[n b]$ & $\sigma^{\text {stat }}$ \\
\hline 4.90 & 0.05 & 0.70 & 0.20 & 24.7863 & 0.806564 \\
\hline 4.90 & 0.05 & 0.90 & 0.20 & 27.8899 & 0.861911 \\
\hline 4.95 & 0.05 & -0.90 & 0.20 & 19.1875 & 0.898599 \\
\hline 4.95 & 0.05 & -0.70 & 0.20 & 19.8208 & 0.929223 \\
\hline 4.95 & 0.05 & -0.50 & 0.20 & 19.2979 & 0.875654 \\
\hline 4.95 & 0.05 & -0.30 & 0.20 & 19.2624 & 0.852319 \\
\hline 4.95 & 0.05 & -0.10 & 0.20 & 21.1977 & o.876947 \\
\hline 4.95 & 0.05 & 0.10 & 0.20 & 22.8880 & 0.904344 \\
\hline 4.95 & 0.05 & 0.30 & 0.20 & 23.9605 & 0.926225 \\
\hline 4.95 & 0.05 & 0.50 & 0.20 & 27.0989 & 0.992264 \\
\hline 4.95 & 0.05 & 0.70 & 0.20 & $29 \cdot 3869$ & 1.056920 \\
\hline 4.95 & 0.05 & 0.90 & 0.20 & 31.6855 & 1.083890 \\
\hline 5.00 & 0.05 & -0.90 & 0.20 & 20.8301 & 0.959492 \\
\hline 5.00 & 0.05 & -0.70 & 0.20 & 21.9218 & 1.000530 \\
\hline 5.00 & 0.05 & -0.50 & 0.20 & 22.2234 & 0.956102 \\
\hline 5.00 & 0.05 & -0.30 & 0.20 & 21.5902 & 0.924299 \\
\hline 5.00 & 0.05 & -0.10 & 0.20 & 22.5261 & 0.916596 \\
\hline 5.00 & 0.05 & 0.10 & 0.20 & 24.2954 & 0.950566 \\
\hline 5.00 & 0.05 & 0.30 & 0.20 & 27.2817 & 1.024130 \\
\hline 5.00 & 0.05 & 0.50 & 0.20 & $29 \cdot 3861$ & 1.070270 \\
\hline 5.00 & 0.05 & 0.70 & 0.20 & 33.0039 & 1.157480 \\
\hline 5.00 & 0.05 & 0.90 & 0.20 & 37.1408 & 1.250910 \\
\hline 5.05 & 0.05 & -0.90 & 0.20 & 19.8681 & 0.818819 \\
\hline 5.05 & 0.05 & -0.70 & 0.20 & 19.0785 & 0.783943 \\
\hline
\end{tabular}


Table A.2 - Continued

\begin{tabular}{|c|c|c|c|c|c|}
\hline$E_{\text {beam }}^{\text {lab }}[\mathrm{GeV}]$ & $\pm d E_{\text {beam }}^{\text {lab }}$ & $\cos \theta_{\bar{p}}^{C . M}$ & $\pm d \cos \theta_{\bar{p}}^{C . M .}$ & $\frac{d \sigma}{d \Omega}[n b]$ & $\sigma^{s t a t}$ \\
\hline 5.05 & 0.05 & -0.50 & 0.20 & 19.9855 & 0.785126 \\
\hline 5.05 & 0.05 & -0.30 & 0.20 & 19.4713 & 0.757079 \\
\hline 5.05 & 0.05 & -0.10 & 0.20 & 20.4877 & 0.768472 \\
\hline 5.05 & 0.05 & 0.10 & 0.20 & 22.1326 & 0.780002 \\
\hline 5.05 & 0.05 & 0.30 & 0.20 & 26.0102 & 0.868978 \\
\hline 5.05 & 0.05 & 0.50 & 0.20 & 27.1433 & 0.887301 \\
\hline 5.05 & 0.05 & 0.70 & 0.20 & 29.9958 & 0.951336 \\
\hline 5.05 & 0.05 & 0.90 & 0.20 & 34.5560 & 1.039250 \\
\hline 5.10 & 0.05 & -0.90 & 0.20 & 20.5182 & 0.855397 \\
\hline 5.10 & 0.05 & -0.70 & 0.20 & 21.2397 & 0.880651 \\
\hline 5.10 & 0.05 & -0.50 & 0.20 & 21.5023 & 0.862302 \\
\hline 5.10 & 0.05 & -0.30 & 0.20 & 22.1137 & 0.855761 \\
\hline 5.10 & 0.05 & -0.10 & 0.20 & 24.1843 & o.889887 \\
\hline 5.10 & 0.05 & 0.10 & 0.20 & $25 \cdot 4528$ & 0.902479 \\
\hline 5.10 & 0.05 & 0.30 & 0.20 & $27 \cdot 3344$ & 0.931285 \\
\hline 5.10 & 0.05 & 0.50 & 0.20 & 31.2890 & 1.025710 \\
\hline 5.10 & 0.05 & 0.70 & 0.20 & 34.6841 & 1.099910 \\
\hline 5.10 & 0.05 & 0.90 & 0.20 & 40.9440 & 1.237570 \\
\hline 5.15 & 0.05 & -0.90 & 0.20 & 22.5785 & 0.975940 \\
\hline 5.15 & 0.05 & -0.70 & 0.20 & 21.6531 & 0.930742 \\
\hline 5.15 & 0.05 & -0.50 & 0.20 & 21.3996 & 0.883340 \\
\hline 5.15 & 0.05 & -0.30 & 0.20 & 22.3707 & 0.892347 \\
\hline 5.15 & 0.05 & -0.10 & 0.20 & 24.1682 & 0.918089 \\
\hline 5.15 & 0.05 & 0.10 & 0.20 & 26.2024 & 0.952884 \\
\hline
\end{tabular}


Table A.2 - Continued

\begin{tabular}{|c|c|c|c|c|c|}
\hline$E_{\text {beam }}^{\text {lab }}[\mathrm{GeV}]$ & $\pm d E_{\text {beam }}^{\text {lab }}$ & $\cos \theta_{\bar{p}}^{C . M}$ & $\pm d \cos \theta_{\bar{p}}^{C . M .}$ & $\frac{d \sigma}{d \Omega}[n b]$ & $\sigma^{\text {stat }}$ \\
\hline 5.15 & 0.05 & 0.30 & 0.20 & 29.1926 & 1.026920 \\
\hline 5.15 & 0.05 & 0.50 & 0.20 & 32.7797 & 1.108120 \\
\hline 5.15 & 0.05 & 0.70 & 0.20 & 36.2393 & 1.191840 \\
\hline 5.15 & 0.05 & 0.90 & 0.20 & 41.0689 & 1.295950 \\
\hline 5.20 & 0.05 & -0.90 & 0.20 & 22.4550 & 0.982175 \\
\hline 5.20 & 0.05 & -0.70 & 0.20 & 22.3397 & 0.959947 \\
\hline 5.20 & 0.05 & -0.50 & 0.20 & 21.4794 & 0.895379 \\
\hline 5.20 & 0.05 & -0.30 & 0.20 & 23.6817 & 0.930157 \\
\hline 5.20 & 0.05 & -0.10 & 0.20 & 24.8183 & 0.939558 \\
\hline 5.20 & 0.05 & 0.10 & 0.20 & 25.8987 & 0.952632 \\
\hline 5.20 & 0.05 & 0.30 & 0.20 & 30.3645 & 1.057380 \\
\hline 5.20 & 0.05 & 0.50 & 0.20 & 31.5493 & 1.071120 \\
\hline 5.20 & 0.05 & 0.70 & 0.20 & 38.2923 & 1.245210 \\
\hline 5.20 & 0.05 & 0.90 & 0.20 & 42.5624 & 1.334730 \\
\hline 5.25 & 0.05 & -0.90 & 0.20 & 24.1966 & 1.177910 \\
\hline 5.25 & 0.05 & -0.70 & 0.20 & $23 \cdot 3409$ & 1.128560 \\
\hline 5.25 & 0.05 & -0.50 & 0.20 & 21.3184 & 1.028970 \\
\hline 5.25 & 0.05 & -0.30 & 0.20 & $23 \cdot 5847$ & 1.060660 \\
\hline 5.25 & 0.05 & -0.10 & 0.20 & 26.4256 & 1.125350 \\
\hline 5.25 & 0.05 & 0.10 & 0.20 & $25 \cdot 5379$ & 1.065050 \\
\hline 5.25 & 0.05 & 0.30 & 0.20 & 28.7136 & 1.147850 \\
\hline 5.25 & 0.05 & 0.50 & 0.20 & 33.8253 & 1.301770 \\
\hline 5.25 & 0.05 & 0.70 & 0.20 & 37.9296 & 1.414380 \\
\hline 5.25 & 0.05 & 0.90 & 0.20 & $44 \cdot 3846$ & 1.581970 \\
\hline
\end{tabular}


Table A.2 - Continued

\begin{tabular}{|c|c|c|c|c|c|}
\hline$E_{\text {beam }}^{\text {lab }}[\mathrm{GeV}]$ & $\pm d E_{\text {beam }}^{l a b}$ & $\cos \theta_{\bar{p}}^{C} \cdot M$ & $\pm d \cos \theta_{\bar{p}}^{C . M}$ & $\frac{d \sigma}{d \Omega}[n b]$ & $\sigma^{\text {stat }}$ \\
\hline $5 \cdot 30$ & 0.05 & -0.90 & 0.20 & 22.4572 & 0.952717 \\
\hline $5 \cdot 30$ & 0.05 & -0.70 & 0.20 & 22.3908 & 0.935649 \\
\hline $5 \cdot 30$ & 0.05 & -0.50 & 0.20 & 24.1840 & 0.935519 \\
\hline $5 \cdot 30$ & 0.05 & -0.30 & 0.20 & 23.0143 & 0.875050 \\
\hline $5 \cdot 30$ & 0.05 & -0.10 & 0.20 & 24.1678 & 0.900497 \\
\hline $5 \cdot 30$ & 0.05 & 0.10 & 0.20 & 27.2378 & 0.959167 \\
\hline $5 \cdot 30$ & 0.05 & 0.30 & 0.20 & 29.6204 & 1.011310 \\
\hline $5 \cdot 30$ & 0.05 & 0.50 & 0.20 & 33.4230 & 1.089230 \\
\hline $5 \cdot 30$ & 0.05 & 0.70 & 0.20 & $39 \cdot 7214$ & 1.244050 \\
\hline $5 \cdot 30$ & 0.05 & 0.90 & 0.20 & 44.6233 & 1.348950 \\
\hline $5 \cdot 35$ & 0.05 & -0.90 & 0.20 & 29.9696 & 1.190190 \\
\hline $5 \cdot 35$ & 0.05 & -0.70 & 0.20 & 25.6396 & 1.059880 \\
\hline $5 \cdot 35$ & 0.05 & -0.50 & 0.20 & 26.3871 & 1.035320 \\
\hline $5 \cdot 35$ & 0.05 & -0.30 & 0.20 & 27.0380 & 1.036060 \\
\hline $5 \cdot 35$ & 0.05 & -0.10 & 0.20 & 28.2997 & 1.038660 \\
\hline $5 \cdot 35$ & 0.05 & 0.10 & 0.20 & 30.0106 & 1.060210 \\
\hline 5.35 & 0.05 & 0.30 & 0.20 & 33.4539 & 1.148320 \\
\hline $5 \cdot 35$ & 0.05 & 0.50 & 0.20 & $39 \cdot 3195$ & 1.283970 \\
\hline $5 \cdot 35$ & 0.05 & 0.70 & 0.20 & 44.5078 & 1.423010 \\
\hline $5 \cdot 35$ & 0.05 & 0.90 & 0.20 & 51.6181 & 1.579150 \\
\hline 5.40 & 0.05 & -0.90 & 0.20 & $24 \cdot 3790$ & 0.992422 \\
\hline 5.40 & 0.05 & -0.70 & 0.20 & 24.2524 & 0.987118 \\
\hline 5.40 & 0.05 & -0.50 & 0.20 & $24 \cdot 3834$ & 0.946359 \\
\hline 5.40 & 0.05 & -0.30 & 0.20 & 25.7648 & 0.959786 \\
\hline
\end{tabular}


Table A.2 - Continued

\begin{tabular}{cccccc}
$E_{\text {beam }}^{\text {lab }}[\mathrm{GeV}]$ & $\pm d E_{\text {beam }}^{\text {lab }}$ & $\cos \theta_{\bar{p}}^{C . M .}$ & $\pm d \cos \theta_{\bar{p}}^{C . M .}$ & $\frac{d \sigma}{d \Omega}[n b]$ & $\sigma^{\text {stat }}$ \\
\hline 5.40 & 0.05 & -0.10 & 0.20 & 24.8927 & 0.911132 \\
5.40 & 0.05 & 0.10 & 0.20 & 28.2325 & 0.978043 \\
5.40 & 0.05 & 0.30 & 0.20 & 31.7214 & 1.058390 \\
5.40 & 0.05 & 0.50 & 0.20 & 34.8211 & 1.130430 \\
5.40 & 0.05 & 0.70 & 0.20 & 42.3941 & 1.321360 \\
5.40 & 0.05 & 0.90 & 0.20 & 50.8705 & 1.515110 \\
\hline
\end{tabular}

A.3 Tabular Data: $d \sigma / d M(p p)$ for $\gamma p \rightarrow p p \bar{p}$

\begin{tabular}{cccccc}
$E_{\text {beam }}^{\text {lab }}[\mathrm{GeV}]$ & $\pm d E_{\text {beam }}^{\text {lab }}$ & $M(p p)\left[\frac{\mathrm{GeV}}{c^{2}}\right]$ & $\pm d M(p p)$ & $\frac{d \sigma}{d M(p p)}[\mathrm{nb}]$ & $\sigma^{\text {stat }}$ \\
\hline 3.95 & 0.10 & 1.94 & 0.01 & 3.1913 & 3.197850 \\
3.95 & 0.10 & 1.95 & 0.01 & 9.4090 & 3.157470 \\
3.95 & 0.10 & 1.96 & 0.01 & 20.1467 & 3.313940 \\
3.95 & 0.10 & 1.97 & 0.01 & 26.0479 & 3.592730 \\
3.95 & 0.10 & 1.98 & 0.01 & 34.1443 & 3.842580 \\
3.95 & 0.10 & 1.99 & 0.01 & 26.9932 & 2.917790 \\
3.95 & 0.10 & 2.00 & 0.01 & 13.2901 & 1.593180 \\
3.95 & 0.10 & 2.01 & 0.01 & 5.2148 & 0.787906 \\
3.95 & 0.10 & 2.02 & 0.01 & 0.4004 & 0.177211 \\
4.05 & 0.10 & 1.92 & 0.01 & 5.3999 & 2.841370 \\
4.05 & 0.10 & 1.93 & 0.01 & 7.9227 & 3.199150 \\
4.05 & 0.10 & 1.94 & 0.01 & 17.6854 & 4.496870 \\
4.05 & 0.10 & 1.95 & 0.01 & 21.5284 & 4.863390 \\
4.05 & 0.10 & 1.96 & 0.01 & 20.9239 & 3.338770 \\
\hline
\end{tabular}


Table A.3 - Continued

\begin{tabular}{|c|c|c|c|c|c|}
\hline$E_{\text {beam }}^{\text {lab }}[\mathrm{GeV}]$ & $\pm d E_{\text {beam }}^{\text {lab }}$ & $M(p p)\left[\frac{G e V}{c^{2}}\right]$ & $\pm d M(p p)$ & $\frac{d \sigma}{d M(p p)}[n b]$ & $\sigma^{\text {stat }}$ \\
\hline 4.05 & 0.10 & 1.97 & 0.01 & $35 \cdot 3467$ & 4.544250 \\
\hline 4.05 & 0.10 & 1.98 & 0.01 & 39.0838 & 4.014550 \\
\hline 4.05 & 0.10 & 1.99 & 0.01 & 43.6591 & $4.14368 \mathrm{o}$ \\
\hline 4.05 & 0.10 & 2.00 & 0.01 & 58.7877 & 4.583010 \\
\hline 4.05 & 0.10 & 2.01 & 0.01 & 51.5041 & 3.720220 \\
\hline 4.05 & 0.10 & 2.02 & 0.01 & 43.1858 & 3.165850 \\
\hline 4.05 & 0.10 & 2.03 & 0.01 & 26.2540 & 2.235090 \\
\hline 4.05 & 0.10 & 2.04 & 0.01 & 11.3263 & 1.464430 \\
\hline 4.05 & 0.10 & 2.05 & 0.01 & 2.3647 & 0.796557 \\
\hline 4.15 & 0.10 & 1.93 & 0.01 & $5 \cdot 4431$ & 1.833010 \\
\hline 4.15 & 0.10 & 1.94 & 0.01 & 13.7200 & 3.094210 \\
\hline 4.15 & 0.10 & 1.95 & 0.01 & 16.8478 & 2.992530 \\
\hline 4.15 & 0.10 & 1.96 & 0.01 & $27 \cdot 4276$ & 4.320030 \\
\hline 4.15 & 0.10 & 1.97 & 0.01 & 35.2836 & 4.005220 \\
\hline 4.15 & 0.10 & 1.98 & 0.01 & 42.7685 & 3.862620 \\
\hline 4.15 & 0.10 & 1.99 & 0.01 & 48.4368 & 4.201330 \\
\hline 4.15 & 0.10 & 2.00 & 0.01 & 53.6022 & 3.909480 \\
\hline 4.15 & 0.10 & 2.01 & 0.01 & 59.9520 & 4.275840 \\
\hline 4.15 & 0.10 & 2.02 & 0.01 & 65.9665 & 4.284040 \\
\hline 4.15 & 0.10 & 2.03 & 0.01 & 63.1989 & 3.901590 \\
\hline 4.15 & 0.10 & 2.04 & 0.01 & 70.2486 & 4.028310 \\
\hline 4.15 & 0.10 & 2.05 & 0.01 & 60.5618 & 3.631370 \\
\hline 4.15 & 0.10 & 2.06 & 0.01 & 38.5143 & 2.711130 \\
\hline 4.15 & 0.10 & 2.07 & 0.01 & 15.6427 & 1.596590 \\
\hline
\end{tabular}


Table A.3 - Continued

\begin{tabular}{|c|c|c|c|c|c|}
\hline$E_{\text {beam }}^{\text {lab }}[\mathrm{GeV}]$ & $\pm d E_{\text {beam }}^{\text {lab }}$ & $M(p p)\left[\frac{G e V}{c^{2}}\right]$ & $\pm d M(p p)$ & $\frac{d \sigma}{d M(p p)}[n b]$ & $\sigma^{\text {stat }}$ \\
\hline 4.15 & 0.10 & 2.08 & 0.01 & 2.3900 & 0.525984 \\
\hline 4.25 & 0.10 & 1.93 & 0.01 & 7.0644 & 1.798560 \\
\hline 4.25 & 0.10 & 1.94 & 0.01 & 10.5899 & 2.158180 \\
\hline 4.25 & 0.10 & 1.95 & 0.01 & 27.1465 & 4.089470 \\
\hline 4.25 & 0.10 & 1.96 & 0.01 & 31.8084 & 4.668940 \\
\hline 4.25 & 0.10 & 1.97 & 0.01 & 34.9323 & 4.502100 \\
\hline 4.25 & 0.10 & 1.98 & 0.01 & 44.8242 & 4.539250 \\
\hline 4.25 & 0.10 & 1.99 & 0.01 & 60.2522 & 4.595240 \\
\hline 4.25 & 0.10 & 2.00 & 0.01 & 56.5651 & 4.360630 \\
\hline 4.25 & 0.10 & 2.01 & 0.01 & 69.8754 & 4.659230 \\
\hline 4.25 & 0.10 & 2.02 & 0.01 & 72.9339 & 4.572410 \\
\hline 4.25 & 0.10 & 2.03 & 0.01 & 71.4403 & 4.345590 \\
\hline 4.25 & 0.10 & 2.04 & 0.01 & 78.7435 & 4.533320 \\
\hline 4.25 & 0.10 & 2.05 & 0.01 & 76.1038 & 4.270890 \\
\hline 4.25 & 0.10 & 2.06 & 0.01 & 85.6891 & 4.536590 \\
\hline 4.25 & 0.10 & 2.07 & 0.01 & 76.9739 & 4.132830 \\
\hline 4.25 & 0.10 & 2.08 & 0.01 & 69.8324 & 3.879710 \\
\hline 4.25 & 0.10 & 2.09 & 0.01 & 43.8407 & 2.858900 \\
\hline 4.25 & 0.10 & 2.10 & 0.01 & 19.8360 & 1.720620 \\
\hline 4.25 & 0.10 & 2.11 & 0.01 & $4 \cdot 5122$ & 0.672510 \\
\hline $4 \cdot 35$ & 0.10 & 1.92 & 0.01 & 2.3078 & 1.178680 \\
\hline $4 \cdot 35$ & 0.10 & 1.93 & 0.01 & 10.0496 & 2.781090 \\
\hline $4 \cdot 35$ & 0.10 & 1.94 & 0.01 & 13.5734 & 2.771490 \\
\hline 4.35 & 0.10 & 1.95 & 0.01 & 23.5083 & 3.455890 \\
\hline
\end{tabular}


Table A.3 - Continued

\begin{tabular}{|c|c|c|c|c|c|}
\hline$E_{\text {beam }}^{\text {lab }}[\mathrm{GeV}]$ & $\pm d E_{\text {beam }}^{\text {lab }}$ & $M(p p)\left[\frac{G e V}{c^{2}}\right]$ & $\pm d M(p p)$ & $\frac{d \sigma}{d M(p p)}[n b]$ & $\sigma^{\text {stat }}$ \\
\hline $4 \cdot 35$ & 0.10 & 1.96 & 0.01 & 37.9990 & 5.036580 \\
\hline $4 \cdot 35$ & 0.10 & 1.97 & 0.01 & 42.3536 & 5.305230 \\
\hline 4.35 & 0.10 & 1.98 & 0.01 & 56.8830 & 5.900290 \\
\hline 4.35 & 0.10 & 1.99 & 0.01 & 58.0398 & 5.593900 \\
\hline 4.35 & 0.10 & 2.00 & 0.01 & 58.7728 & 5.067780 \\
\hline $4 \cdot 35$ & 0.10 & 2.01 & 0.01 & 73.8568 & 5.575720 \\
\hline $4 \cdot 35$ & 0.10 & 2.02 & 0.01 & 63.6780 & 4.812850 \\
\hline $4 \cdot 35$ & 0.10 & 2.03 & 0.01 & 84.3065 & 5.774750 \\
\hline $4 \cdot 35$ & 0.10 & 2.04 & 0.01 & 82.9553 & 5.514690 \\
\hline $4 \cdot 35$ & 0.10 & 2.05 & 0.01 & 89.4037 & 5.683990 \\
\hline $4 \cdot 35$ & 0.10 & 2.06 & 0.01 & 99.7110 & 5.913440 \\
\hline $4 \cdot 35$ & 0.10 & 2.07 & 0.01 & 96.1752 & 5.737900 \\
\hline $4 \cdot 35$ & 0.10 & 2.08 & 0.01 & 104.3340 & 5.967000 \\
\hline $4 \cdot 35$ & 0.10 & 2.09 & 0.01 & 89.2476 & 5.360360 \\
\hline $4 \cdot 35$ & 0.10 & 2.10 & 0.01 & 81.7457 & 4.765510 \\
\hline $4 \cdot 35$ & 0.10 & 2.11 & 0.01 & 79.4879 & 4.623350 \\
\hline $4 \cdot 35$ & 0.10 & 2.12 & 0.01 & 54.6309 & 3.749610 \\
\hline $4 \cdot 35$ & 0.10 & 2.13 & 0.01 & 20.1691 & 1.929120 \\
\hline $4 \cdot 35$ & 0.10 & 2.14 & 0.01 & 4.7732 & 0.760519 \\
\hline 4.45 & 0.10 & 1.92 & 0.01 & 1.8824 & 0.961329 \\
\hline 4.45 & 0.10 & 1.93 & 0.01 & 10.2740 & 2.472110 \\
\hline $4 \cdot 45$ & 0.10 & 1.94 & 0.01 & 13.7954 & 2.435860 \\
\hline $4 \cdot 45$ & 0.10 & 1.95 & 0.01 & 22.7464 & 2.894900 \\
\hline 4.45 & 0.10 & 1.96 & 0.01 & 37.1089 & 3.903800 \\
\hline
\end{tabular}


Table A.3 - Continued

\begin{tabular}{|c|c|c|c|c|c|}
\hline$E_{\text {beam }}^{\text {lab }}[\mathrm{GeV}]$ & $\pm d E_{\text {beam }}^{\text {lab }}$ & $M(p p)\left[\frac{G e V}{c^{2}}\right]$ & $\pm d M(p p)$ & $\frac{d \sigma}{d M(p p)}[n b]$ & $\sigma^{\text {stat }}$ \\
\hline $4 \cdot 45$ & 0.10 & 1.97 & 0.01 & 33.2125 & 3.605030 \\
\hline $4 \cdot 45$ & 0.10 & 1.98 & 0.01 & 47.8764 & 4.371520 \\
\hline $4 \cdot 45$ & 0.10 & 1.99 & 0.01 & 56.3819 & 4.608460 \\
\hline 4.45 & 0.10 & 2.00 & 0.01 & 64.1530 & 4.856120 \\
\hline $4 \cdot 45$ & 0.10 & 2.01 & 0.01 & 68.2411 & 4.458600 \\
\hline 4.45 & 0.10 & 2.02 & 0.01 & 73.7362 & $4.66908 \mathrm{o}$ \\
\hline 4.45 & 0.10 & 2.03 & 0.01 & 74.8970 & 4.401050 \\
\hline 4.45 & 0.10 & 2.04 & 0.01 & 80.7688 & 4.408750 \\
\hline 4.45 & 0.10 & 2.05 & 0.01 & 83.3742 & 4.555940 \\
\hline 4.45 & 0.10 & 2.06 & 0.01 & 105.7390 & $5.22888 \mathrm{o}$ \\
\hline 4.45 & 0.10 & 2.07 & 0.01 & 96.8858 & 4.749520 \\
\hline 4.45 & 0.10 & 2.08 & 0.01 & 96.8354 & 4.744530 \\
\hline 4.45 & 0.10 & 2.09 & 0.01 & 103.7870 & $4.76818 \mathrm{o}$ \\
\hline 4.45 & 0.10 & 2.10 & 0.01 & 99.7521 & 4.588340 \\
\hline 4.45 & 0.10 & 2.11 & 0.01 & 110.0890 & 4.956860 \\
\hline 4.45 & 0.10 & 2.12 & 0.01 & 100.3470 & 4.535790 \\
\hline 4.45 & 0.10 & 2.13 & 0.01 & 90.6925 & 4.224010 \\
\hline 4.45 & 0.10 & 2.14 & 0.01 & 77.2060 & 3.635290 \\
\hline $4 \cdot 45$ & 0.10 & 2.15 & 0.01 & $54 \cdot 7936$ & 2.984350 \\
\hline 4.45 & 0.10 & 2.16 & 0.01 & 25.4877 & 1.844070 \\
\hline $4 \cdot 45$ & 0.10 & 2.17 & 0.01 & $7 \cdot 9155$ & 0.984783 \\
\hline $4 \cdot 55$ & 0.10 & 1.92 & 0.01 & 3.2633 & 1.220870 \\
\hline $4 \cdot 55$ & 0.10 & 1.93 & 0.01 & 14.8261 & 2.848700 \\
\hline 4.55 & 0.10 & 1.94 & 0.01 & 16.6407 & 2.612520 \\
\hline
\end{tabular}


Table A.3 - Continued

\begin{tabular}{|c|c|c|c|c|c|}
\hline$E_{\text {beam }}^{\text {lab }}[\mathrm{GeV}]$ & $\pm d E_{\text {beam }}^{\text {lab }}$ & $M(p p)\left[\frac{G e V}{c^{2}}\right]$ & $\pm d M(p p)$ & $\frac{d \sigma}{d M(p p)}[n b]$ & $\sigma^{\text {stat }}$ \\
\hline 4.55 & 0.10 & 1.95 & 0.01 & 30.9520 & 3.676440 \\
\hline 4.55 & 0.10 & 1.96 & 0.01 & 32.6881 & 3.701330 \\
\hline 4.55 & 0.10 & 1.97 & 0.01 & $37.075^{2}$ & 4.098440 \\
\hline 4.55 & 0.10 & 1.98 & 0.01 & 43.0864 & 4.513840 \\
\hline $4 \cdot 55$ & 0.10 & 1.99 & 0.01 & 62.5832 & 5.097220 \\
\hline 4.55 & 0.10 & 2.00 & 0.01 & 62.1019 & 5.090370 \\
\hline $4 \cdot 55$ & 0.10 & 2.01 & 0.01 & 70.0319 & 5.198540 \\
\hline 4.55 & 0.10 & 2.02 & 0.01 & 74.6681 & 5.061240 \\
\hline 4.55 & 0.10 & 2.03 & 0.01 & 75.4037 & 4.726360 \\
\hline 4.55 & 0.10 & 2.04 & 0.01 & 80.8317 & 4.705530 \\
\hline 4.55 & 0.10 & 2.05 & 0.01 & 82.6868 & 4.628760 \\
\hline $4 \cdot 55$ & 0.10 & 2.06 & 0.01 & 91.9023 & 5.017450 \\
\hline $4 \cdot 55$ & 0.10 & 2.07 & 0.01 & 92.5926 & 4.901730 \\
\hline $4 \cdot 55$ & 0.10 & 2.08 & 0.01 & 107.1470 & 5.395540 \\
\hline 4.55 & 0.10 & 2.09 & 0.01 & 104.5960 & 5.337440 \\
\hline 4.55 & 0.10 & 2.10 & 0.01 & 113.0680 & 5.566000 \\
\hline 4.55 & 0.10 & 2.11 & 0.01 & 111.6280 & 5.204970 \\
\hline $4 \cdot 55$ & 0.10 & 2.12 & 0.01 & 105.6660 & 5.091910 \\
\hline $4 \cdot 55$ & 0.10 & 2.13 & 0.01 & 106.4180 & 4.988330 \\
\hline $4 \cdot 55$ & 0.10 & 2.14 & 0.01 & 110.5230 & 5.044300 \\
\hline 4.55 & 0.10 & 2.15 & 0.01 & 102.6400 & $4.70788 \mathrm{o}$ \\
\hline $4 \cdot 55$ & 0.10 & 2.16 & 0.01 & 85.8538 & 4.098350 \\
\hline $4 \cdot 55$ & 0.10 & 2.17 & 0.01 & 88.2094 & 4.418420 \\
\hline 4.55 & 0.10 & 2.18 & 0.01 & 56.2325 & 3.161790 \\
\hline
\end{tabular}


Table A.3 - Continued

\begin{tabular}{|c|c|c|c|c|c|}
\hline$E_{\text {beam }}^{\text {lab }}[\mathrm{GeV}]$ & $\pm d E_{\text {beam }}^{\text {lab }}$ & $M(p p)\left[\frac{G e V}{c^{2}}\right]$ & $\pm d M(p p)$ & $\frac{d \sigma}{d M(p p)}[n b]$ & $\sigma^{\text {stat }}$ \\
\hline 4.55 & 0.10 & 2.19 & 0.01 & $35 \cdot 3636$ & 2.449620 \\
\hline 4.55 & 0.10 & 2.20 & 0.01 & 10.3722 & $1.18528 \mathrm{o}$ \\
\hline 4.65 & 0.10 & 1.92 & 0.01 & 5.4020 & 1.774290 \\
\hline 4.65 & 0.10 & 1.93 & 0.01 & 11.0333 & 2.683800 \\
\hline 4.65 & 0.10 & 1.94 & 0.01 & 17.5416 & 2.809630 \\
\hline 4.65 & 0.10 & 1.95 & 0.01 & 21.9780 & 3.019390 \\
\hline 4.65 & 0.10 & 1.96 & 0.01 & 30.8154 & 3.421610 \\
\hline 4.65 & 0.10 & 1.97 & 0.01 & 32.1410 & 3.728050 \\
\hline 4.65 & 0.10 & 1.98 & 0.01 & 47.1240 & 4.256810 \\
\hline 4.65 & 0.10 & 1.99 & 0.01 & 50.5737 & 4.513710 \\
\hline 4.65 & 0.10 & 2.00 & 0.01 & 55.8928 & 4.648330 \\
\hline 4.65 & 0.10 & 2.01 & 0.01 & 68.4090 & 5.031270 \\
\hline 4.65 & 0.10 & 2.02 & 0.01 & 59.4100 & 4.474750 \\
\hline 4.65 & 0.10 & 2.03 & 0.01 & 75.0676 & 4.838870 \\
\hline 4.65 & 0.10 & 2.04 & 0.01 & 78.2487 & 4.826340 \\
\hline 4.65 & 0.10 & 2.05 & 0.01 & 81.5152 & 4.629250 \\
\hline 4.65 & 0.10 & 2.06 & 0.01 & 97.8881 & 5.297640 \\
\hline 4.65 & 0.10 & 2.07 & 0.01 & 100.2380 & 5.054060 \\
\hline 4.65 & 0.10 & 2.08 & 0.01 & 86.1680 & 4.727910 \\
\hline 4.65 & 0.10 & 2.09 & 0.01 & 97.0068 & 4.955510 \\
\hline 4.65 & 0.10 & 2.10 & 0.01 & $95 \cdot 3402$ & 4.711820 \\
\hline 4.65 & 0.10 & 2.11 & 0.01 & 110.2640 & 5.308630 \\
\hline 4.65 & 0.10 & 2.12 & 0.01 & 106.3130 & 5.221820 \\
\hline 4.65 & 0.10 & 2.13 & 0.01 & 117.4560 & 5.573350 \\
\hline
\end{tabular}


Table A.3 - Continued

\begin{tabular}{|c|c|c|c|c|c|}
\hline$E_{\text {beam }}^{\text {lab }}[\mathrm{GeV}]$ & $\pm d E_{\text {beam }}^{\text {lab }}$ & $M(p p)\left[\frac{G e V}{c^{2}}\right]$ & $\pm d M(p p)$ & $\frac{d \sigma}{d M(p p)}[n b]$ & $\sigma^{\text {stat }}$ \\
\hline 4.65 & 0.10 & 2.14 & 0.01 & 116.3370 & 5.318820 \\
\hline 4.65 & 0.10 & 2.15 & 0.01 & 110.1420 & 5.103530 \\
\hline 4.65 & 0.10 & 2.16 & 0.01 & 111.2550 & 5.016930 \\
\hline 4.65 & 0.10 & 2.17 & 0.01 & 115.5470 & 5.070400 \\
\hline 4.65 & 0.10 & 2.18 & 0.01 & 114.2480 & $5.08468 \mathrm{o}$ \\
\hline 4.65 & 0.10 & 2.19 & 0.01 & 88.7761 & 4.328530 \\
\hline 4.65 & 0.10 & 2.20 & 0.01 & 87.8545 & 4.167650 \\
\hline 4.65 & 0.10 & 2.21 & 0.01 & 54.9060 & 3.016270 \\
\hline 4.65 & 0.10 & 2.22 & 0.01 & 29.8059 & 2.209230 \\
\hline 4.65 & 0.10 & 2.23 & 0.01 & 8.5643 & 1.042030 \\
\hline 4.65 & 0.10 & 2.24 & 0.01 & 0.0402 & 0.024782 \\
\hline $4 \cdot 75$ & 0.10 & 1.92 & 0.01 & $4 \cdot 5391$ & 2.399810 \\
\hline $4 \cdot 75$ & 0.10 & 1.93 & 0.01 & 7.6380 & 1.587670 \\
\hline $4 \cdot 75$ & 0.10 & 1.94 & 0.01 & 17.8642 & 3.012490 \\
\hline 4.75 & 0.10 & 1.95 & 0.01 & 22.6460 & 3.535320 \\
\hline $4 \cdot 75$ & 0.10 & 1.96 & 0.01 & 31.7096 & 3.667550 \\
\hline 4.75 & 0.10 & 1.97 & 0.01 & 40.1769 & 4.486870 \\
\hline $4 \cdot 75$ & 0.10 & 1.98 & 0.01 & 62.2979 & 5.530740 \\
\hline $4 \cdot 75$ & 0.10 & 1.99 & 0.01 & 59.7624 & 5.250700 \\
\hline $4 \cdot 75$ & 0.10 & 2.00 & 0.01 & 62.1757 & 5.226590 \\
\hline $4 \cdot 75$ & 0.10 & 2.01 & 0.01 & 66.4583 & 5.370380 \\
\hline $4 \cdot 75$ & 0.10 & 2.02 & 0.01 & 76.9443 & 5.712860 \\
\hline $4 \cdot 75$ & 0.10 & 2.03 & 0.01 & 79.1327 & 5.576070 \\
\hline 4.75 & 0.10 & 2.04 & 0.01 & 80.3627 & 5.459810 \\
\hline
\end{tabular}


Table A.3 - Continued

\begin{tabular}{|c|c|c|c|c|c|}
\hline$E_{\text {beam }}^{\text {lab }}[\mathrm{GeV}]$ & $\pm d E_{\text {beam }}^{\text {lab }}$ & $M(p p)\left[\frac{G e V}{c^{2}}\right]$ & $\pm d M(p p)$ & $\frac{d \sigma}{d M(p p)}[n b]$ & $\sigma^{\text {stat }}$ \\
\hline 4.75 & 0.10 & 2.05 & 0.01 & 98.6691 & 6.168970 \\
\hline 4.75 & 0.10 & 2.06 & 0.01 & 93.2465 & 5.743760 \\
\hline $4 \cdot 75$ & 0.10 & 2.07 & 0.01 & 96.4463 & 5.586500 \\
\hline 4.75 & 0.10 & 2.08 & 0.01 & 102.7350 & 5.728540 \\
\hline 4.75 & 0.10 & 2.09 & 0.01 & 104.8340 & 5.854330 \\
\hline $4 \cdot 75$ & 0.10 & 2.10 & 0.01 & 119.8470 & 6.285130 \\
\hline $4 \cdot 75$ & 0.10 & 2.11 & 0.01 & 121.9430 & 6.174430 \\
\hline $4 \cdot 75$ & 0.10 & 2.12 & 0.01 & 107.6550 & 5.612610 \\
\hline 4.75 & 0.10 & 2.13 & 0.01 & 120.0260 & 5.925530 \\
\hline $4 \cdot 75$ & 0.10 & 2.14 & 0.01 & 123.8990 & 6.007410 \\
\hline 4.75 & 0.10 & 2.15 & 0.01 & 144.4660 & 6.871420 \\
\hline $4 \cdot 75$ & 0.10 & 2.16 & 0.01 & 140.1660 & 6.663800 \\
\hline $4 \cdot 75$ & 0.10 & 2.17 & 0.01 & 137.7640 & 6.359040 \\
\hline $4 \cdot 75$ & 0.10 & 2.18 & 0.01 & 136.7020 & 6.261240 \\
\hline 4.75 & 0.10 & 2.19 & 0.01 & 113.1450 & 5.305760 \\
\hline $4 \cdot 75$ & 0.10 & 2.20 & 0.01 & 123.3620 & 5.705790 \\
\hline $4 \cdot 75$ & 0.10 & 2.21 & 0.01 & 119.4020 & 5.575210 \\
\hline $4 \cdot 75$ & 0.10 & 2.22 & 0.01 & 100.6210 & 4.874600 \\
\hline $4 \cdot 75$ & 0.10 & 2.23 & 0.01 & 92.8699 & 4.584040 \\
\hline $4 \cdot 75$ & 0.10 & 2.24 & 0.01 & 59.7139 & 3.524810 \\
\hline $4 \cdot 75$ & 0.10 & 2.25 & 0.01 & 35.6244 & 2.665740 \\
\hline $4 \cdot 75$ & 0.10 & 2.26 & 0.01 & 10.7003 & 1.332080 \\
\hline $4 \cdot 75$ & 0.10 & 2.27 & 0.01 & 0.0696 & 0.042476 \\
\hline 4.85 & 0.10 & 1.92 & 0.01 & 13.2848 & 4.928460 \\
\hline
\end{tabular}


Table A.3 - Continued

\begin{tabular}{|c|c|c|c|c|c|}
\hline$E_{\text {beam }}^{\text {lab }}[\mathrm{GeV}]$ & $\pm d E_{\text {beam }}^{\text {lab }}$ & $M(p p)\left[\frac{G e V}{c^{2}}\right]$ & $\pm d M(p p)$ & $\frac{d \sigma}{d M(p p)}[n b]$ & $\sigma^{\text {stat }}$ \\
\hline 4.85 & 0.10 & 1.93 & 0.01 & 16.9167 & 2.695620 \\
\hline 4.85 & 0.10 & 1.94 & 0.01 & 10.4438 & 1.932460 \\
\hline 4.85 & 0.10 & 1.95 & 0.01 & 16.4461 & 2.449980 \\
\hline 4.85 & 0.10 & 1.96 & 0.01 & 25.1964 & 2.889170 \\
\hline 4.85 & 0.10 & 1.97 & 0.01 & 40.2675 & 3.836840 \\
\hline 4.85 & 0.10 & 1.98 & 0.01 & 40.1927 & 3.612050 \\
\hline 4.85 & 0.10 & 1.99 & 0.01 & 47.9792 & $4.16618 \mathrm{o}$ \\
\hline 4.85 & 0.10 & 2.00 & 0.01 & 48.8483 & 4.165450 \\
\hline 4.85 & 0.10 & 2.01 & 0.01 & 60.7875 & 4.450560 \\
\hline 4.85 & 0.10 & 2.02 & 0.01 & 66.7664 & 4.825620 \\
\hline 4.85 & 0.10 & 2.03 & 0.01 & 67.6022 & 4.820950 \\
\hline 4.85 & 0.10 & 2.04 & 0.01 & 77.0396 & 4.859330 \\
\hline 4.85 & 0.10 & 2.05 & 0.01 & 73.9165 & 4.680370 \\
\hline 4.85 & 0.10 & 2.06 & 0.01 & 72.1252 & 4.476410 \\
\hline 4.85 & 0.10 & 2.07 & 0.01 & 91.2401 & $5.07328 \mathrm{o}$ \\
\hline 4.85 & 0.10 & 2.08 & 0.01 & 89.5748 & 4.700600 \\
\hline 4.85 & 0.10 & 2.09 & 0.01 & 91.5445 & 4.715340 \\
\hline 4.85 & 0.10 & 2.10 & 0.01 & 98.0512 & 4.919380 \\
\hline 4.85 & 0.10 & 2.11 & 0.01 & 101.7780 & 4.870150 \\
\hline 4.85 & 0.10 & 2.12 & 0.01 & 102.5620 & 4.864650 \\
\hline 4.85 & 0.10 & 2.13 & 0.01 & 120.8670 & 5.562650 \\
\hline 4.85 & 0.10 & 2.14 & 0.01 & 114.2500 & 5.226190 \\
\hline 4.85 & 0.10 & 2.15 & 0.01 & 119.4060 & $5 \cdot 313700$ \\
\hline 4.85 & 0.10 & 2.16 & 0.01 & 103.9760 & 4.844180 \\
\hline
\end{tabular}


Table A.3 - Continued

\begin{tabular}{|c|c|c|c|c|c|}
\hline$E_{\text {beam }}^{\text {lab }}[\mathrm{GeV}]$ & $\pm d E_{\text {beam }}^{\text {lab }}$ & $M(p p)\left[\frac{G e V}{c^{2}}\right]$ & $\pm d M(p p)$ & $\frac{d \sigma}{d M(p p)}[n b]$ & $\sigma^{\text {stat }}$ \\
\hline 4.85 & 0.10 & 2.17 & 0.01 & 112.3230 & 5.111990 \\
\hline 4.85 & 0.10 & 2.18 & 0.01 & 118.5680 & 5.286470 \\
\hline 4.85 & 0.10 & 2.19 & 0.01 & 124.9970 & 5.624630 \\
\hline 4.85 & 0.10 & 2.20 & 0.01 & 120.3480 & 5.166470 \\
\hline 4.85 & 0.10 & 2.21 & 0.01 & 110.9640 & 4.775080 \\
\hline 4.85 & 0.10 & 2.22 & 0.01 & 107.7690 & 4.752570 \\
\hline 4.85 & 0.10 & 2.23 & 0.01 & 110.2510 & $4.71848 \mathrm{o}$ \\
\hline 4.85 & 0.10 & 2.24 & 0.01 & 106.1570 & 4.581760 \\
\hline 4.85 & 0.10 & 2.25 & 0.01 & 101.8140 & 4.466510 \\
\hline 4.85 & 0.10 & 2.26 & 0.01 & 80.9684 & 3.881940 \\
\hline 4.85 & 0.10 & 2.27 & 0.01 & 52.0730 & 2.942270 \\
\hline 4.85 & 0.10 & 2.28 & 0.01 & 33.2606 & 2.254210 \\
\hline 4.85 & 0.10 & 2.29 & 0.01 & 7.6056 & $0.92144^{2}$ \\
\hline 4.95 & 0.10 & 1.92 & 0.01 & 2.9744 & 1.073590 \\
\hline 4.95 & 0.10 & 1.93 & 0.01 & 17.0888 & 3.299800 \\
\hline 4.95 & 0.10 & 1.94 & 0.01 & 12.4593 & 2.244820 \\
\hline 4.95 & 0.10 & 1.95 & 0.01 & 14.1800 & $2.31428 \mathrm{o}$ \\
\hline 4.95 & 0.10 & 1.96 & 0.01 & 24.1462 & 3.133140 \\
\hline 4.95 & 0.10 & 1.97 & 0.01 & 37.0252 & 3.719210 \\
\hline 4.95 & 0.10 & 1.98 & 0.01 & 45.3959 & 4.489170 \\
\hline 4.95 & 0.10 & 1.99 & 0.01 & 47.8970 & 4.497930 \\
\hline 4.95 & 0.10 & 2.00 & 0.01 & 56.0922 & 5.083380 \\
\hline 4.95 & 0.10 & 2.01 & 0.01 & 58.7161 & 5.218060 \\
\hline 4.95 & 0.10 & 2.02 & 0.01 & 76.6717 & 5.899230 \\
\hline
\end{tabular}


Table A.3 - Continued

\begin{tabular}{|c|c|c|c|c|c|}
\hline$E_{\text {beam }}^{\text {lab }}[\mathrm{GeV}]$ & $\pm d E_{\text {beam }}^{\text {lab }}$ & $M(p p)\left[\frac{G e V}{c^{2}}\right]$ & $\pm d M(p p)$ & $\frac{d \sigma}{d M(p p)}[n b]$ & $\sigma^{\text {stat }}$ \\
\hline 4.95 & 0.10 & 2.03 & 0.01 & 75.8250 & 5.629710 \\
\hline 4.95 & 0.10 & 2.04 & 0.01 & 83.3128 & 5.843950 \\
\hline 4.95 & 0.10 & 2.05 & 0.01 & 80.3727 & 5.704230 \\
\hline 4.95 & 0.10 & 2.06 & 0.01 & 86.7706 & 5.508720 \\
\hline 4.95 & 0.10 & 2.07 & 0.01 & 84.3564 & 5.315250 \\
\hline 4.95 & 0.10 & 2.08 & 0.01 & 86.9601 & $5 \cdot 312100$ \\
\hline 4.95 & 0.10 & 2.09 & 0.01 & 102.4470 & 5.899380 \\
\hline 4.95 & 0.10 & 2.10 & 0.01 & 98.5642 & 5.425210 \\
\hline 4.95 & 0.10 & 2.11 & 0.01 & 107.8870 & 5.643430 \\
\hline 4.95 & 0.10 & 2.12 & 0.01 & 101.1700 & 5.402980 \\
\hline 4.95 & 0.10 & 2.13 & 0.01 & 122.5760 & $6.16698 \mathrm{o}$ \\
\hline 4.95 & 0.10 & 2.14 & 0.01 & 115.7860 & 6.173850 \\
\hline 4.95 & 0.10 & 2.15 & 0.01 & 122.5630 & 6.131100 \\
\hline 4.95 & 0.10 & 2.16 & 0.01 & 112.7320 & 5.717320 \\
\hline 4.95 & 0.10 & 2.17 & 0.01 & 122.1360 & 5.932570 \\
\hline 4.95 & 0.10 & 2.18 & 0.01 & 137.2110 & 6.471800 \\
\hline 4.95 & 0.10 & 2.19 & 0.01 & 126.7390 & 6.068200 \\
\hline 4.95 & 0.10 & 2.20 & 0.01 & 134.3000 & 6.236980 \\
\hline 4.95 & 0.10 & 2.21 & 0.01 & 125.8600 & 5.931090 \\
\hline 4.95 & 0.10 & 2.22 & 0.01 & 129.9810 & 6.151420 \\
\hline 4.95 & 0.10 & 2.23 & 0.01 & 131.8330 & 6.037830 \\
\hline 4.95 & 0.10 & 2.24 & 0.01 & 125.7760 & 5.784190 \\
\hline 4.95 & 0.10 & 2.25 & 0.01 & 136.2390 & 6.055790 \\
\hline 4.95 & 0.10 & 2.26 & 0.01 & 119.1570 & $5 \cdot 524840$ \\
\hline
\end{tabular}


Table A.3 - Continued

\begin{tabular}{|c|c|c|c|c|c|}
\hline$E_{\text {beam }}^{\text {lab }}[\mathrm{GeV}]$ & $\pm d E_{\text {beam }}^{\text {lab }}$ & $M(p p)\left[\frac{G e V}{c^{2}}\right]$ & $\pm d M(p p)$ & $\frac{d \sigma}{d M(p p)}[n b]$ & $\sigma^{\text {stat }}$ \\
\hline 4.95 & 0.10 & 2.27 & 0.01 & 112.4660 & 5.353360 \\
\hline 4.95 & 0.10 & 2.28 & 0.01 & 91.9319 & 4.532800 \\
\hline 4.95 & 0.10 & 2.29 & 0.01 & 88.0299 & 4.576210 \\
\hline 4.95 & 0.10 & 2.30 & 0.01 & 67.8764 & 3.929090 \\
\hline 4.95 & 0.10 & 2.31 & 0.01 & 30.4763 & 2.389300 \\
\hline 4.95 & 0.10 & 2.32 & 0.01 & 8.4094 & 1.142820 \\
\hline 5.05 & 0.10 & 1.92 & 0.01 & 5.0538 & 1.672210 \\
\hline 5.05 & 0.10 & 1.93 & 0.01 & 8.6703 & 1.823950 \\
\hline 5.05 & 0.10 & 1.94 & 0.01 & 11.2110 & 1.905920 \\
\hline 5.05 & 0.10 & 1.95 & 0.01 & 21.1749 & 3.052830 \\
\hline 5.05 & 0.10 & 1.96 & 0.01 & 24.9875 & $2.87778 \mathrm{o}$ \\
\hline 5.05 & 0.10 & 1.97 & 0.01 & 29.0826 & 3.199380 \\
\hline 5.05 & 0.10 & 1.98 & 0.01 & 31.5488 & 3.310630 \\
\hline 5.05 & 0.10 & 1.99 & 0.01 & 40.9767 & 4.011450 \\
\hline 5.05 & 0.10 & 2.00 & 0.01 & $49 \cdot 3110$ & 4.214930 \\
\hline 5.05 & 0.10 & 2.01 & 0.01 & 64.9173 & 4.880540 \\
\hline 5.05 & 0.10 & 2.02 & 0.01 & 66.6627 & 5.197980 \\
\hline 5.05 & 0.10 & 2.03 & 0.01 & 69.6218 & 5.094210 \\
\hline 5.05 & 0.10 & 2.04 & 0.01 & 64.0303 & 4.514840 \\
\hline 5.05 & 0.10 & 2.05 & 0.01 & 76.3452 & 4.815340 \\
\hline 5.05 & 0.10 & 2.06 & 0.01 & 72.6435 & 4.604490 \\
\hline 5.05 & 0.10 & 2.07 & 0.01 & 83.6946 & 4.962340 \\
\hline 5.05 & 0.10 & 2.08 & 0.01 & 78.1484 & 4.673250 \\
\hline 5.05 & 0.10 & 2.09 & 0.01 & 87.6173 & 4.845730 \\
\hline
\end{tabular}


Table A.3 - Continued

\begin{tabular}{|c|c|c|c|c|c|}
\hline$E_{\text {beam }}^{\text {lab }}[\mathrm{GeV}]$ & $\pm d E_{\text {beam }}^{\text {lab }}$ & $M(p p)\left[\frac{G e V}{c^{2}}\right]$ & $\pm d M(p p)$ & $\frac{d \sigma}{d M(p p)}[n b]$ & $\sigma^{\text {stat }}$ \\
\hline 5.05 & 0.10 & 2.10 & 0.01 & 86.2554 & 4.709080 \\
\hline 5.05 & 0.10 & 2.11 & 0.01 & 100.1310 & 5.294790 \\
\hline 5.05 & 0.10 & 2.12 & 0.01 & 99.6670 & 5.035280 \\
\hline 5.05 & 0.10 & 2.13 & 0.01 & 109.0360 & 5.368700 \\
\hline 5.05 & 0.10 & 2.14 & 0.01 & 115.2750 & 5.413350 \\
\hline 5.05 & 0.10 & 2.15 & 0.01 & $113 \cdot 3100$ & 5.355580 \\
\hline 5.05 & 0.10 & 2.16 & 0.01 & 118.1890 & 5.629650 \\
\hline 5.05 & 0.10 & 2.17 & 0.01 & 106.7820 & 5.013850 \\
\hline 5.05 & 0.10 & 2.18 & 0.01 & 108.3680 & 5.164660 \\
\hline 5.05 & 0.10 & 2.19 & 0.01 & 119.2060 & 5.481670 \\
\hline 5.05 & 0.10 & 2.20 & 0.01 & 118.8770 & 5.392240 \\
\hline 5.05 & 0.10 & 2.21 & 0.01 & 120.0850 & 5.317340 \\
\hline 5.05 & 0.10 & 2.22 & 0.01 & 126.0090 & 5.561400 \\
\hline 5.05 & 0.10 & 2.23 & 0.01 & 129.5530 & $5 \cdot 772160$ \\
\hline 5.05 & 0.10 & 2.24 & 0.01 & 122.4250 & $5 \cdot 35048 \mathrm{o}$ \\
\hline 5.05 & 0.10 & 2.25 & 0.01 & 119.0820 & 5.223540 \\
\hline 5.05 & 0.10 & 2.26 & 0.01 & 120.6510 & 5.220040 \\
\hline 5.05 & 0.10 & 2.27 & 0.01 & 121.9730 & 5.193850 \\
\hline 5.05 & 0.10 & 2.28 & 0.01 & 122.4980 & 5.227550 \\
\hline 5.05 & 0.10 & 2.29 & 0.01 & 109.4460 & 4.871080 \\
\hline 5.05 & 0.10 & 2.30 & 0.01 & 94.9693 & 4.384340 \\
\hline 5.05 & 0.10 & 2.31 & 0.01 & 97.2009 & 4.399980 \\
\hline 5.05 & 0.10 & 2.32 & 0.01 & 72.5702 & 3.636240 \\
\hline 5.05 & 0.10 & 2.33 & 0.01 & 51.2514 & 2.985660 \\
\hline
\end{tabular}


Table A.3 - Continued

\begin{tabular}{|c|c|c|c|c|c|}
\hline$E_{\text {beam }}^{\text {lab }}[\mathrm{GeV}]$ & $\pm d E_{\text {beam }}^{\text {lab }}$ & $M(p p)\left[\frac{G e V}{c^{2}}\right]$ & $\pm d M(p p)$ & $\frac{d \sigma}{d M(p p)}[n b]$ & $\sigma^{\text {stat }}$ \\
\hline 5.05 & 0.10 & 2.34 & 0.01 & 26.5376 & 2.078640 \\
\hline 5.05 & 0.10 & 2.35 & 0.01 & 5.8505 & 0.785894 \\
\hline 5.15 & 0.10 & 1.92 & 0.01 & 2.5998 & 0.841170 \\
\hline 5.15 & 0.10 & 1.93 & 0.01 & 11.8709 & 2.230790 \\
\hline 5.15 & 0.10 & 1.94 & 0.01 & 11.9691 & 2.060040 \\
\hline 5.15 & 0.10 & 1.95 & 0.01 & 16.9447 & 2.477870 \\
\hline 5.15 & 0.10 & 1.96 & 0.01 & 18.3373 & $2.47348 \mathrm{o}$ \\
\hline 5.15 & 0.10 & 1.97 & 0.01 & 30.4264 & 3.551060 \\
\hline 5.15 & 0.10 & 1.98 & 0.01 & 32.7922 & 3.436920 \\
\hline 5.15 & 0.10 & 1.99 & 0.01 & 52.0413 & 4.694220 \\
\hline 5.15 & 0.10 & 2.00 & 0.01 & 45.7985 & $4.47028 \mathrm{o}$ \\
\hline 5.15 & 0.10 & 2.01 & 0.01 & 51.0893 & 4.323210 \\
\hline 5.15 & 0.10 & 2.02 & 0.01 & 59.6426 & 4.791260 \\
\hline 5.15 & 0.10 & 2.03 & 0.01 & 64.3309 & 5.153130 \\
\hline 5.15 & 0.10 & 2.04 & 0.01 & 72.8149 & 5.367570 \\
\hline 5.15 & 0.10 & 2.05 & 0.01 & 68.6633 & 4.918460 \\
\hline 5.15 & 0.10 & 2.06 & 0.01 & 79.4585 & $5 \cdot 372080$ \\
\hline 5.15 & 0.10 & 2.07 & 0.01 & 83.6605 & 5.522580 \\
\hline 5.15 & 0.10 & 2.08 & 0.01 & 76.4582 & 4.962100 \\
\hline 5.15 & 0.10 & 2.09 & 0.01 & 94.4201 & 5.575630 \\
\hline 5.15 & 0.10 & 2.10 & 0.01 & 87.6863 & 5.257870 \\
\hline 5.15 & 0.10 & 2.11 & 0.01 & 94.0911 & 5.447970 \\
\hline 5.15 & 0.10 & 2.12 & 0.01 & 100.4520 & 5.530220 \\
\hline 5.15 & 0.10 & 2.13 & 0.01 & 100.4100 & 5.337810 \\
\hline
\end{tabular}


Table A.3 - Continued

\begin{tabular}{|c|c|c|c|c|c|}
\hline$E_{\text {beam }}^{\text {lab }}[\mathrm{GeV}]$ & $\pm d E_{\text {beam }}^{\text {lab }}$ & $M(p p)\left[\frac{G e V}{c^{2}}\right]$ & $\pm d M(p p)$ & $\frac{d \sigma}{d M(p p)}[n b]$ & $\sigma^{\text {stat }}$ \\
\hline 5.15 & 0.10 & 2.14 & 0.01 & 98.3977 & 5.494550 \\
\hline 5.15 & 0.10 & 2.15 & 0.01 & 100.4310 & 5.256780 \\
\hline 5.15 & 0.10 & 2.16 & 0.01 & 112.6250 & 5.767320 \\
\hline 5.15 & 0.10 & 2.17 & 0.01 & 110.0070 & 5.676830 \\
\hline 5.15 & 0.10 & 2.18 & 0.01 & 118.3650 & 5.865660 \\
\hline 5.15 & 0.10 & 2.19 & 0.01 & 122.4110 & 5.973190 \\
\hline 5.15 & 0.10 & 2.20 & 0.01 & 119.7950 & 5.749580 \\
\hline 5.15 & 0.10 & 2.21 & 0.01 & 118.5510 & 5.604580 \\
\hline 5.15 & 0.10 & 2.22 & 0.01 & 120.9540 & 5.662100 \\
\hline 5.15 & 0.10 & 2.23 & 0.01 & 117.1490 & 5.725110 \\
\hline 5.15 & 0.10 & 2.24 & 0.01 & 121.4400 & 5.737620 \\
\hline 5.15 & 0.10 & 2.25 & 0.01 & 112.3030 & 5.348130 \\
\hline 5.15 & 0.10 & 2.26 & 0.01 & 128.7820 & 5.847410 \\
\hline 5.15 & 0.10 & 2.27 & 0.01 & 125.6400 & 5.727760 \\
\hline 5.15 & 0.10 & 2.28 & 0.01 & 123.2160 & 5.669400 \\
\hline 5.15 & 0.10 & 2.29 & 0.01 & 117.5310 & 5.367970 \\
\hline 5.15 & 0.10 & 2.30 & 0.01 & 109.2230 & 4.956610 \\
\hline 5.15 & 0.10 & 2.31 & 0.01 & 125.9920 & 5.593140 \\
\hline 5.15 & 0.10 & 2.32 & 0.01 & 115.2760 & 5.368870 \\
\hline 5.15 & 0.10 & 2.33 & 0.01 & 110.5660 & 5.123270 \\
\hline 5.15 & 0.10 & 2.34 & 0.01 & 93.8016 & 4.548460 \\
\hline 5.15 & 0.10 & 2.35 & 0.01 & 79.6237 & 4.254220 \\
\hline 5.15 & 0.10 & 2.36 & 0.01 & $45 \cdot 3528$ & $2.97128 \mathrm{o}$ \\
\hline 5.15 & 0.10 & 2.37 & 0.01 & $25 \cdot 7456$ & 2.124490 \\
\hline
\end{tabular}


Table A.3 - Continued

\begin{tabular}{|c|c|c|c|c|c|}
\hline$E_{\text {beam }}^{\text {lab }}[\mathrm{GeV}]$ & $\pm d E_{\text {beam }}^{\text {lab }}$ & $M(p p)\left[\frac{\mathrm{GeV}}{c^{2}}\right]$ & $\pm d M(p p)$ & $\frac{d \sigma}{d M(p p)}[n b]$ & $\sigma^{\text {stat }}$ \\
\hline 5.15 & 0.10 & 2.38 & 0.01 & 4.9141 & 0.747095 \\
\hline 5.25 & 0.10 & 1.92 & 0.01 & 3.0563 & 1.364180 \\
\hline 5.25 & 0.10 & 1.93 & 0.01 & $7 \cdot 3341$ & 1.721470 \\
\hline 5.25 & 0.10 & 1.94 & 0.01 & 14.2748 & 2.546050 \\
\hline 5.25 & 0.10 & 1.95 & 0.01 & 13.6978 & 2.414850 \\
\hline 5.25 & 0.10 & 1.96 & 0.01 & 19.6409 & 2.806350 \\
\hline 5.25 & 0.10 & 1.97 & 0.01 & 26.6072 & 3.029650 \\
\hline 5.25 & 0.10 & 1.98 & 0.01 & 28.2432 & 3.275500 \\
\hline 5.25 & 0.10 & 1.99 & 0.01 & 28.8859 & 3.222820 \\
\hline 5.25 & 0.10 & 2.00 & 0.01 & 37.6142 & 3.746600 \\
\hline 5.25 & 0.10 & 2.01 & 0.01 & 46.7479 & 4.349590 \\
\hline 5.25 & 0.10 & 2.02 & 0.01 & 54.8835 & 4.980800 \\
\hline 5.25 & 0.10 & 2.03 & 0.01 & 62.5084 & 5.412790 \\
\hline 5.25 & 0.10 & 2.04 & 0.01 & 74.0245 & $6.10848 \mathrm{o}$ \\
\hline 5.25 & 0.10 & 2.05 & 0.01 & 69.9401 & 5.399500 \\
\hline 5.25 & 0.10 & 2.06 & 0.01 & $77 \cdot 3165$ & 5.702760 \\
\hline 5.25 & 0.10 & 2.07 & 0.01 & 68.3689 & 5.204640 \\
\hline 5.25 & 0.10 & 2.08 & 0.01 & 65.1250 & 4.895160 \\
\hline 5.25 & 0.10 & 2.09 & 0.01 & 78.2177 & 5.212300 \\
\hline 5.25 & 0.10 & 2.10 & 0.01 & 79.2438 & 5.277390 \\
\hline 5.25 & 0.10 & 2.11 & 0.01 & 85.3913 & 5.434570 \\
\hline 5.25 & 0.10 & 2.12 & 0.01 & 89.8951 & 5.467670 \\
\hline 5.25 & 0.10 & 2.13 & 0.01 & 94.5482 & 5.720630 \\
\hline 5.25 & 0.10 & 2.14 & 0.01 & 88.7565 & 5.299200 \\
\hline
\end{tabular}


Table A.3 - Continued

\begin{tabular}{|c|c|c|c|c|c|}
\hline$E_{\text {beam }}^{\text {lab }}[\mathrm{GeV}]$ & $\pm d E_{\text {beam }}^{\text {lab }}$ & $M(p p)\left[\frac{G e V}{c^{2}}\right]$ & $\pm d M(p p)$ & $\frac{d \sigma}{d M(p p)}[n b]$ & $\sigma^{\text {stat }}$ \\
\hline 5.25 & 0.10 & 2.15 & 0.01 & 99.8520 & 5.605470 \\
\hline 5.25 & 0.10 & 2.16 & 0.01 & 95.0338 & 5.461400 \\
\hline 5.25 & 0.10 & 2.17 & 0.01 & $107 \cdot 5190$ & 5.781100 \\
\hline 5.25 & 0.10 & 2.18 & 0.01 & 103.9820 & 5.793890 \\
\hline 5.25 & 0.10 & 2.19 & 0.01 & 108.6490 & 5.805600 \\
\hline 5.25 & 0.10 & 2.20 & 0.01 & 113.1690 & 5.880530 \\
\hline 5.25 & 0.10 & 2.21 & 0.01 & 101.6220 & 5.585710 \\
\hline 5.25 & 0.10 & 2.22 & 0.01 & 119.7640 & 6.179710 \\
\hline 5.25 & 0.10 & 2.23 & 0.01 & 118.1920 & 6.008150 \\
\hline 5.25 & 0.10 & 2.24 & 0.01 & 112.2080 & 5.708470 \\
\hline 5.25 & 0.10 & 2.25 & 0.01 & 126.3440 & 6.159970 \\
\hline 5.25 & 0.10 & 2.26 & 0.01 & 124.7780 & 6.144740 \\
\hline 5.25 & 0.10 & 2.27 & 0.01 & 136.0710 & 6.527450 \\
\hline 5.25 & 0.10 & 2.28 & 0.01 & $117 \cdot 5400$ & $5.85618 \mathrm{o}$ \\
\hline 5.25 & 0.10 & 2.29 & 0.01 & 123.4670 & 6.006100 \\
\hline 5.25 & 0.10 & 2.30 & 0.01 & 125.8400 & 6.017400 \\
\hline 5.25 & 0.10 & 2.31 & 0.01 & 128.7570 & 6.236040 \\
\hline 5.25 & 0.10 & 2.32 & 0.01 & 120.8870 & 5.839470 \\
\hline 5.25 & 0.10 & 2.33 & 0.01 & 104.2870 & 5.292300 \\
\hline 5.25 & 0.10 & 2.34 & 0.01 & 115.4380 & 5.682740 \\
\hline 5.25 & 0.10 & 2.35 & 0.01 & 100.3430 & 5.042060 \\
\hline 5.25 & 0.10 & 2.36 & 0.01 & 103.5690 & 5.167360 \\
\hline 5.25 & 0.10 & 2.37 & 0.01 & 88.8494 & 4.821730 \\
\hline 5.25 & 0.10 & 2.38 & 0.01 & 67.4943 & 3.898810 \\
\hline
\end{tabular}


Table A.3 - Continued

\begin{tabular}{|c|c|c|c|c|c|}
\hline$E_{\text {beam }}^{\text {lab }}[\mathrm{GeV}]$ & $\pm d E_{\text {beam }}^{\text {lab }}$ & $M(p p)\left[\frac{G e V}{c^{2}}\right]$ & $\pm d M(p p)$ & $\frac{d \sigma}{d M(p p)}[n b]$ & $\sigma^{\text {stat }}$ \\
\hline 5.25 & 0.10 & 2.39 & 0.01 & $43 \cdot 7841$ & 3.012150 \\
\hline 5.25 & 0.10 & 2.40 & 0.01 & 17.8699 & 1.766970 \\
\hline 5.25 & 0.10 & 2.41 & 0.01 & 1.8370 & 0.375892 \\
\hline 5.35 & 0.10 & 1.92 & 0.01 & 3.1221 & 1.226210 \\
\hline $5 \cdot 35$ & 0.10 & 1.93 & 0.01 & $7 \cdot 3112$ & $1.65378 \mathrm{o}$ \\
\hline $5 \cdot 35$ & 0.10 & 1.94 & 0.01 & 15.6266 & 2.693650 \\
\hline $5 \cdot 35$ & 0.10 & 1.95 & 0.01 & 17.1785 & 2.641230 \\
\hline $5 \cdot 35$ & 0.10 & 1.96 & 0.01 & 17.2014 & 2.206950 \\
\hline $5 \cdot 35$ & 0.10 & 1.97 & 0.01 & 22.2350 & 2.820160 \\
\hline $5 \cdot 35$ & 0.10 & 1.98 & 0.01 & 24.3300 & 2.815070 \\
\hline 5.35 & 0.10 & 1.99 & 0.01 & 40.1107 & 4.151440 \\
\hline $5 \cdot 35$ & 0.10 & 2.00 & 0.01 & 34.5910 & 3.575240 \\
\hline $5 \cdot 35$ & 0.10 & 2.01 & 0.01 & 46.7725 & 4.321940 \\
\hline $5 \cdot 35$ & 0.10 & 2.02 & 0.01 & 49.7353 & 4.383060 \\
\hline $5 \cdot 35$ & 0.10 & 2.03 & 0.01 & 61.2102 & 5.061210 \\
\hline $5 \cdot 35$ & 0.10 & 2.04 & 0.01 & 64.5431 & 5.227540 \\
\hline $5 \cdot 35$ & 0.10 & 2.05 & 0.01 & 78.4076 & 5.691710 \\
\hline $5 \cdot 35$ & 0.10 & 2.06 & 0.01 & 70.3190 & $5 \cdot 312500$ \\
\hline $5 \cdot 35$ & 0.10 & 2.07 & 0.01 & 72.7563 & 5.170910 \\
\hline $5 \cdot 35$ & 0.10 & 2.08 & 0.01 & 72.8128 & 5.286650 \\
\hline $5 \cdot 35$ & 0.10 & 2.09 & 0.01 & 84.2893 & 5.550630 \\
\hline $5 \cdot 35$ & 0.10 & 2.10 & 0.01 & 89.0949 & 5.615080 \\
\hline $5 \cdot 35$ & 0.10 & 2.11 & 0.01 & 88.4388 & 5.460140 \\
\hline 5.35 & 0.10 & 2.12 & 0.01 & 89.2638 & 5.271130 \\
\hline
\end{tabular}


Table A.3 - Continued

\begin{tabular}{|c|c|c|c|c|c|}
\hline$E_{\text {beam }}^{\text {lab }}[\mathrm{GeV}]$ & $\pm d E_{\text {beam }}^{\text {lab }}$ & $M(p p)\left[\frac{G e V}{c^{2}}\right]$ & $\pm d M(p p)$ & $\frac{d \sigma}{d M(p p)}[n b]$ & $\sigma^{\text {stat }}$ \\
\hline 5.35 & 0.10 & 2.13 & 0.01 & $97 \cdot 4172$ & 5.704950 \\
\hline 5.35 & 0.10 & 2.14 & 0.01 & 93.6978 & $5 \cdot 37228 \mathrm{o}$ \\
\hline $5 \cdot 35$ & 0.10 & 2.15 & 0.01 & 101.2920 & 5.588240 \\
\hline 5.35 & 0.10 & 2.16 & 0.01 & 91.9440 & 5.026570 \\
\hline $5 \cdot 35$ & 0.10 & 2.17 & 0.01 & 98.0808 & 5.305130 \\
\hline $5 \cdot 35$ & 0.10 & 2.18 & 0.01 & 107.5640 & 5.596550 \\
\hline $5 \cdot 35$ & 0.10 & 2.19 & 0.01 & 113.8850 & 5.804340 \\
\hline $5 \cdot 35$ & 0.10 & 2.20 & 0.01 & 121.1810 & 6.076380 \\
\hline $5 \cdot 35$ & 0.10 & 2.21 & 0.01 & 107.1000 & 5.480300 \\
\hline $5 \cdot 35$ & 0.10 & 2.22 & 0.01 & $124 \cdot 3250$ & 6.022920 \\
\hline $5 \cdot 35$ & 0.10 & 2.23 & 0.01 & 113.4690 & 5.728990 \\
\hline $5 \cdot 35$ & 0.10 & 2.24 & 0.01 & 121.0930 & 5.881240 \\
\hline $5 \cdot 35$ & 0.10 & 2.25 & 0.01 & 123.8080 & 5.957140 \\
\hline $5 \cdot 35$ & 0.10 & 2.26 & 0.01 & 121.3350 & 5.838750 \\
\hline $5 \cdot 35$ & 0.10 & 2.27 & 0.01 & 124.8710 & 5.753750 \\
\hline $5 \cdot 35$ & 0.10 & 2.28 & 0.01 & 129.1230 & 5.994340 \\
\hline $5 \cdot 35$ & 0.10 & 2.29 & 0.01 & 124.6790 & 5.957830 \\
\hline $5 \cdot 35$ & 0.10 & 2.30 & 0.01 & 120.4690 & 5.631630 \\
\hline $5 \cdot 35$ & 0.10 & 2.31 & 0.01 & 120.7790 & 5.707980 \\
\hline $5 \cdot 35$ & 0.10 & 2.32 & 0.01 & 125.8780 & 5.875270 \\
\hline $5 \cdot 35$ & 0.10 & 2.33 & 0.01 & 118.1270 & 5.543720 \\
\hline $5 \cdot 35$ & 0.10 & 2.34 & 0.01 & 121.1430 & 5.636630 \\
\hline $5 \cdot 35$ & 0.10 & 2.35 & 0.01 & 128.4800 & 5.680450 \\
\hline $5 \cdot 35$ & 0.10 & 2.36 & 0.01 & 123.6720 & 5.551940 \\
\hline
\end{tabular}


Table A.3 - Continued

\begin{tabular}{cccccc}
$E_{\text {beam }}^{\text {lab }}[\mathrm{GeV}]$ & $\pm d E_{\text {beam }}^{\text {lab }}$ & $M(p p)\left[\frac{\mathrm{GeV}}{c^{2}}\right]$ & $\pm d M(p p)$ & $\frac{d \sigma}{d M(p p)}[n b]$ & $\sigma^{\text {stat }}$ \\
\hline 5.35 & 0.10 & 2.37 & 0.01 & 118.8900 & 5.481830 \\
5.35 & 0.10 & 2.38 & 0.01 & 103.2810 & 4.900250 \\
5.35 & 0.10 & 2.39 & 0.01 & 95.3797 & 4.693750 \\
5.35 & 0.10 & 2.40 & 0.01 & 82.4645 & 4.343160 \\
5.35 & 0.10 & 2.41 & 0.01 & 63.2517 & 3.619990 \\
5.35 & 0.10 & 2.42 & 0.01 & 33.5681 & 2.550610 \\
5.35 & 0.10 & 2.43 & 0.01 & 14.2263 & 1.434880 \\
5.35 & 0.10 & 2.44 & 0.01 & 1.8157 & 0.378286 \\
\hline
\end{tabular}

A.4 Tabular Data: $d \sigma / d M(p \bar{p})$ for $\gamma p \rightarrow p p \bar{p}$

\begin{tabular}{cccccc}
$E_{\text {beam }}^{\text {lab }}[\mathrm{GeV}]$ & $\pm d E_{\text {beam }}^{\text {lab }}$ & $M(p \bar{p})\left[\frac{\mathrm{GeV}}{c^{2}}\right]$ & $\pm d M(p \bar{p})$ & $\frac{d \sigma}{d M(p \bar{p})}[n b]$ & $\sigma^{\text {stat }}$ \\
\hline 3.95 & 0.10 & 1.92 & 0.01 & 8.1669 & 1.864860 \\
3.95 & 0.10 & 1.93 & 0.01 & 47.3201 & 5.024970 \\
3.95 & 0.10 & 1.94 & 0.01 & 68.4964 & 5.544900 \\
3.95 & 0.10 & 1.95 & 0.01 & 61.5021 & 5.457000 \\
3.95 & 0.10 & 1.96 & 0.01 & 63.1083 & 5.809180 \\
3.95 & 0.10 & 1.97 & 0.01 & 57.3055 & 5.411320 \\
3.95 & 0.10 & 1.98 & 0.01 & 46.7733 & 5.441810 \\
3.95 & 0.10 & 1.99 & 0.01 & 13.3207 & 3.665890 \\
3.95 & 0.10 & 2.00 & 0.01 & 4.0623 & 2.045950 \\
4.05 & 0.10 & 1.92 & 0.01 & 10.7557 & 1.618350 \\
4.05 & 0.10 & 1.93 & 0.01 & 62.3277 & 4.820800 \\
4.05 & 0.10 & 1.94 & 0.01 & 95.9350 & 6.305800 \\
\hline
\end{tabular}


Table A.4 - Continued

\begin{tabular}{|c|c|c|c|c|c|}
\hline$E_{\text {beam }}^{\text {lab }}[\mathrm{GeV}]$ & $\pm d E_{\text {beam }}^{\text {lab }}$ & $M(p \bar{p})\left[\frac{G e V}{c^{2}}\right]$ & $\pm d M(p \bar{p})$ & $\frac{d \sigma}{d M(p \bar{p})}[n b]$ & $\sigma^{\text {stat }}$ \\
\hline 4.05 & 0.10 & 1.95 & 0.01 & 104.2580 & 6.547640 \\
\hline 4.05 & 0.10 & 1.96 & 0.01 & 119.0400 & 6.971790 \\
\hline 4.05 & 0.10 & 1.97 & 0.01 & 120.3920 & 6.843050 \\
\hline 4.05 & 0.10 & 1.98 & 0.01 & 104.8410 & 6.609660 \\
\hline 4.05 & 0.10 & 1.99 & 0.01 & 93.2872 & 6.235850 \\
\hline 4.05 & 0.10 & 2.00 & 0.01 & 77.6097 & 5.983260 \\
\hline 4.05 & 0.10 & 2.01 & 0.01 & 54.9770 & 6.077430 \\
\hline 4.05 & 0.10 & 2.02 & 0.01 & 26.5995 & 5.008240 \\
\hline 4.05 & 0.10 & 2.03 & 0.01 & 13.5526 & 3.949070 \\
\hline 4.15 & 0.10 & 1.92 & 0.01 & 15.8923 & 1.914760 \\
\hline 4.15 & 0.10 & 1.93 & 0.01 & 67.2110 & 4.371950 \\
\hline 4.15 & 0.10 & 1.94 & 0.01 & 114.3900 & 6.348190 \\
\hline 4.15 & 0.10 & 1.95 & 0.01 & 135.9720 & 6.919180 \\
\hline 4.15 & 0.10 & 1.96 & 0.01 & 127.0200 & $6.23038 \mathrm{o}$ \\
\hline 4.15 & 0.10 & 1.97 & 0.01 & 134.1520 & 6.724730 \\
\hline 4.15 & 0.10 & 1.98 & 0.01 & 145.9440 & 7.102010 \\
\hline 4.15 & 0.10 & 1.99 & 0.01 & 140.6210 & 6.954970 \\
\hline 4.15 & 0.10 & 2.00 & 0.01 & 126.8780 & 6.516770 \\
\hline 4.15 & 0.10 & 2.01 & 0.01 & 121.8590 & 6.400440 \\
\hline 4.15 & 0.10 & 2.02 & 0.01 & 108.1250 & 6.183180 \\
\hline 4.15 & 0.10 & 2.03 & 0.01 & 96.3651 & 6.561710 \\
\hline 4.15 & 0.10 & 2.04 & 0.01 & 44.0018 & 4.642670 \\
\hline 4.15 & 0.10 & 2.05 & 0.01 & 31.0545 & 4.654970 \\
\hline 4.15 & 0.10 & 2.06 & 0.01 & 9.9860 & 3.361910 \\
\hline
\end{tabular}


Table A.4 - Continued

\begin{tabular}{|c|c|c|c|c|c|}
\hline$E_{\text {beam }}^{\text {lab }}[\mathrm{GeV}]$ & $\pm d E_{\text {beam }}^{\text {lab }}$ & $M(p \bar{p})\left[\frac{G e V}{c^{2}}\right]$ & $\pm d M(p \bar{p})$ & $\frac{d \sigma}{d M(p \bar{p})}[n b]$ & $\sigma^{\text {stat }}$ \\
\hline 4.25 & 0.10 & 1.92 & 0.01 & 14.2528 & 1.752740 \\
\hline 4.25 & 0.10 & 1.93 & 0.01 & 70.5824 & 4.519250 \\
\hline 4.25 & 0.10 & 1.94 & 0.01 & 121.7740 & 6.310190 \\
\hline 4.25 & 0.10 & 1.95 & 0.01 & 143.1020 & $6.83408 \mathrm{o}$ \\
\hline 4.25 & 0.10 & 1.96 & 0.01 & 153.3230 & 6.906990 \\
\hline 4.25 & 0.10 & 1.97 & 0.01 & $157 \cdot 3410$ & $7.09668 \mathrm{o}$ \\
\hline 4.25 & 0.10 & 1.98 & 0.01 & 155.2720 & 6.997220 \\
\hline 4.25 & 0.10 & 1.99 & 0.01 & 162.7010 & 7.279930 \\
\hline 4.25 & 0.10 & 2.00 & 0.01 & $177 \cdot 5530$ & 7.684740 \\
\hline 4.25 & 0.10 & 2.01 & 0.01 & 154.1130 & 7.002890 \\
\hline 4.25 & 0.10 & 2.02 & 0.01 & 145.8850 & 6.881620 \\
\hline 4.25 & 0.10 & 2.03 & 0.01 & 159.4980 & $7 \cdot 310270$ \\
\hline 4.25 & 0.10 & 2.04 & 0.01 & 137.6620 & 6.694980 \\
\hline 4.25 & 0.10 & 2.05 & 0.01 & 107.8130 & 6.315720 \\
\hline 4.25 & 0.10 & 2.06 & 0.01 & 88.6524 & 6.318710 \\
\hline 4.25 & 0.10 & 2.07 & 0.01 & 73.1957 & 5.997780 \\
\hline 4.25 & 0.10 & 2.08 & 0.01 & 34.4778 & $4.54488 \mathrm{o}$ \\
\hline 4.25 & 0.10 & 2.09 & 0.01 & 21.9909 & 4.746270 \\
\hline 4.25 & 0.10 & 2.10 & 0.01 & 8.7999 & 4.548940 \\
\hline $4 \cdot 35$ & 0.10 & 1.92 & 0.01 & 13.1295 & 1.730210 \\
\hline $4 \cdot 35$ & 0.10 & 1.93 & 0.01 & 69.7502 & 5.003990 \\
\hline $4 \cdot 35$ & 0.10 & 1.94 & 0.01 & 124.1960 & $7 \cdot 323540$ \\
\hline $4 \cdot 35$ & 0.10 & 1.95 & 0.01 & 149.7130 & 7.934270 \\
\hline 4.35 & 0.10 & 1.96 & 0.01 & 184.4400 & 9.079990 \\
\hline
\end{tabular}


Table A.4 - Continued

\begin{tabular}{|c|c|c|c|c|c|}
\hline$E_{\text {beam }}^{\text {lab }}[\mathrm{GeV}]$ & $\pm d E_{\text {beam }}^{\text {lab }}$ & $M(p \bar{p})\left[\frac{G e V}{c^{2}}\right]$ & $\pm d M(p \bar{p})$ & $\frac{d \sigma}{d M(p \bar{p})}[n b]$ & $\sigma^{\text {stat }}$ \\
\hline $4 \cdot 35$ & 0.10 & 1.97 & 0.01 & 166.9830 & 8.219790 \\
\hline 4.35 & 0.10 & 1.98 & 0.01 & 176.9040 & 8.409870 \\
\hline 4.35 & 0.10 & 1.99 & 0.01 & 178.1760 & 8.435900 \\
\hline $4 \cdot 35$ & 0.10 & 2.00 & 0.01 & 194.6740 & 8.990600 \\
\hline $4 \cdot 35$ & 0.10 & 2.01 & 0.01 & 205.4040 & 9.453560 \\
\hline $4 \cdot 35$ & 0.10 & 2.02 & 0.01 & 181.2160 & 8.805180 \\
\hline $4 \cdot 35$ & 0.10 & 2.03 & 0.01 & 189.3130 & 9.143390 \\
\hline $4 \cdot 35$ & 0.10 & 2.04 & 0.01 & 179.0010 & 8.851940 \\
\hline $4 \cdot 35$ & 0.10 & 2.05 & 0.01 & 166.3360 & 8.513890 \\
\hline $4 \cdot 35$ & 0.10 & 2.06 & 0.01 & 150.4140 & $7 \cdot 796140$ \\
\hline $4 \cdot 35$ & 0.10 & 2.07 & 0.01 & 138.8270 & $7 \cdot 738010$ \\
\hline $4 \cdot 35$ & 0.10 & 2.08 & 0.01 & 113.0140 & 7.267950 \\
\hline $4 \cdot 35$ & 0.10 & 2.09 & 0.01 & 92.5710 & 7.054800 \\
\hline $4 \cdot 35$ & 0.10 & 2.10 & 0.01 & 77.9742 & 7.047300 \\
\hline $4 \cdot 35$ & 0.10 & 2.11 & 0.01 & 46.7500 & 6.190740 \\
\hline $4 \cdot 35$ & 0.10 & 2.12 & 0.01 & 21.0665 & 4.977030 \\
\hline $4 \cdot 35$ & 0.10 & 2.13 & 0.01 & 17.3838 & 6.131220 \\
\hline 4.45 & 0.10 & 1.92 & 0.01 & 20.9021 & 2.100330 \\
\hline 4.45 & 0.10 & 1.93 & 0.01 & 75.2850 & 4.382070 \\
\hline 4.45 & 0.10 & 1.94 & 0.01 & 123.4920 & 5.592610 \\
\hline 4.45 & 0.10 & 1.95 & 0.01 & 146.5880 & 6.180690 \\
\hline 4.45 & 0.10 & 1.96 & 0.01 & 176.0140 & 6.920350 \\
\hline 4.45 & 0.10 & 1.97 & 0.01 & 167.8230 & 6.547030 \\
\hline 4.45 & 0.10 & 1.98 & 0.01 & 193.4530 & 7.185470 \\
\hline
\end{tabular}


Table A.4 - Continued

\begin{tabular}{|c|c|c|c|c|c|}
\hline$E_{\text {beam }}^{\text {lab }}[\mathrm{GeV}]$ & $\pm d E_{\text {beam }}^{\text {lab }}$ & $M(p \bar{p})\left[\frac{G e V}{c^{2}}\right]$ & $\pm d M(p \bar{p})$ & $\frac{d \sigma}{d M(p \bar{p})}[n b]$ & $\sigma^{\text {stat }}$ \\
\hline 4.45 & 0.10 & 1.99 & 0.01 & $187 \cdot 7880$ & 7.056580 \\
\hline $4 \cdot 45$ & 0.10 & 2.00 & 0.01 & 189.3140 & 7.075810 \\
\hline $4 \cdot 45$ & 0.10 & 2.01 & 0.01 & 200.1810 & $7 \cdot 339560$ \\
\hline $4 \cdot 45$ & 0.10 & 2.02 & 0.01 & 204.4470 & 7.436370 \\
\hline 4.45 & 0.10 & 2.03 & 0.01 & 202.4750 & 7.387350 \\
\hline 4.45 & 0.10 & 2.04 & 0.01 & 209.0570 & 7.678820 \\
\hline $4 \cdot 45$ & 0.10 & 2.05 & 0.01 & 191.8950 & 7.274490 \\
\hline 4.45 & 0.10 & 2.06 & 0.01 & 181.5460 & 6.896140 \\
\hline 4.45 & 0.10 & 2.07 & 0.01 & 156.6660 & 6.385210 \\
\hline 4.45 & 0.10 & 2.08 & 0.01 & 157.5620 & 6.316890 \\
\hline 4.45 & 0.10 & 2.09 & 0.01 & 157.5140 & 6.750540 \\
\hline 4.45 & 0.10 & 2.10 & 0.01 & 138.1020 & 6.449320 \\
\hline 4.45 & 0.10 & 2.11 & 0.01 & $117 \cdot 7280$ & 6.185380 \\
\hline 4.45 & 0.10 & 2.12 & 0.01 & 110.2930 & 6.490430 \\
\hline 4.45 & 0.10 & 2.13 & 0.01 & 68.9181 & 5.354320 \\
\hline 4.45 & 0.10 & 2.14 & 0.01 & 46.6170 & 5.216050 \\
\hline 4.45 & 0.10 & 2.15 & 0.01 & 25.4064 & $4 \cdot 421700$ \\
\hline 4.45 & 0.10 & 2.16 & 0.01 & 11.5596 & 5.901530 \\
\hline $4 \cdot 55$ & 0.10 & 1.92 & 0.01 & 22.3513 & $2.09738 \mathrm{o}$ \\
\hline 4.55 & 0.10 & 1.93 & 0.01 & 83.4714 & 4.804720 \\
\hline 4.55 & 0.10 & 1.94 & 0.01 & 121.8100 & 6.033200 \\
\hline $4 \cdot 55$ & 0.10 & 1.95 & 0.01 & 152.5290 & 6.785160 \\
\hline $4 \cdot 55$ & 0.10 & 1.96 & 0.01 & 169.3490 & 7.032500 \\
\hline 4.55 & 0.10 & 1.97 & 0.01 & 183.8150 & $7 \cdot 405370$ \\
\hline
\end{tabular}


Table A.4 - Continued

\begin{tabular}{|c|c|c|c|c|c|}
\hline$E_{\text {beam }}^{\text {lab }}[\mathrm{GeV}]$ & $\pm d E_{\text {beam }}^{\text {lab }}$ & $M(p \bar{p})\left[\frac{G e V}{c^{2}}\right]$ & $\pm d M(p \bar{p})$ & $\frac{d \sigma}{d M(p \bar{p})}[n b]$ & $\sigma^{\text {stat }}$ \\
\hline $4 \cdot 55$ & 0.10 & 1.98 & 0.01 & 193.6110 & 7.630280 \\
\hline 4.55 & 0.10 & 1.99 & 0.01 & 204.7780 & $7 \cdot 746020$ \\
\hline 4.55 & 0.10 & 2.00 & 0.01 & 185.7870 & 7.203240 \\
\hline 4.55 & 0.10 & 2.01 & 0.01 & 209.2750 & 7.966810 \\
\hline 4.55 & 0.10 & 2.02 & 0.01 & 234.9100 & 8.573250 \\
\hline $4 \cdot 55$ & 0.10 & 2.03 & 0.01 & 216.2140 & 8.012280 \\
\hline $4 \cdot 55$ & 0.10 & 2.04 & 0.01 & 207.9650 & $7 \cdot 740950$ \\
\hline 4.55 & 0.10 & 2.05 & 0.01 & 207.2820 & $7 \cdot 910150$ \\
\hline $4 \cdot 55$ & 0.10 & 2.06 & 0.01 & 211.3080 & 7.861000 \\
\hline 4.55 & 0.10 & 2.07 & 0.01 & 194.2150 & 7.621020 \\
\hline 4.55 & 0.10 & 2.08 & 0.01 & 193.1450 & $7 \cdot 569160$ \\
\hline 4.55 & 0.10 & 2.09 & 0.01 & 172.7990 & 7.244930 \\
\hline $4 \cdot 55$ & 0.10 & 2.10 & 0.01 & 166.5370 & 7.097010 \\
\hline $4 \cdot 55$ & 0.10 & 2.11 & 0.01 & 173.7570 & $7 \cdot 454670$ \\
\hline 4.55 & 0.10 & 2.12 & 0.01 & 160.3800 & 7.260980 \\
\hline 4.55 & 0.10 & 2.13 & 0.01 & 143.2660 & 7.252310 \\
\hline 4.55 & 0.10 & 2.14 & 0.01 & 114.2880 & 6.375190 \\
\hline 4.55 & 0.10 & 2.15 & 0.01 & 91.1658 & 6.086830 \\
\hline 4.55 & 0.10 & 2.16 & 0.01 & 68.9680 & 6.042490 \\
\hline 4.55 & 0.10 & 2.17 & 0.01 & 46.8637 & 5.997750 \\
\hline $4 \cdot 55$ & 0.10 & 2.18 & 0.01 & 16.0596 & 3.305610 \\
\hline 4.55 & 0.10 & 2.19 & 0.01 & 6.8732 & $3 \cdot 518890$ \\
\hline 4.65 & 0.10 & 1.92 & 0.01 & $25 \cdot 3750$ & 2.460330 \\
\hline 4.65 & 0.10 & 1.93 & 0.01 & 80.2757 & 4.743060 \\
\hline
\end{tabular}


Table A.4 - Continued

\begin{tabular}{|c|c|c|c|c|c|}
\hline$E_{\text {beam }}^{l a b}[\mathrm{GeV}]$ & $\pm d E_{\text {beam }}^{l a b}$ & $M(p \bar{p})\left[\frac{G e V}{c^{2}}\right]$ & $\pm d M(p \bar{p})$ & $\frac{d \sigma}{d M(p \bar{p})}[n b]$ & $\sigma^{\text {stat }}$ \\
\hline 4.65 & 0.10 & 1.94 & 0.01 & 115.7290 & 5.680090 \\
\hline 4.65 & 0.10 & 1.95 & 0.01 & 150.3320 & 6.806990 \\
\hline 4.65 & 0.10 & 1.96 & 0.01 & 162.3820 & 6.813200 \\
\hline 4.65 & 0.10 & 1.97 & 0.01 & 197.2020 & 7.831180 \\
\hline 4.65 & 0.10 & 1.98 & 0.01 & 198.3460 & $7 \cdot 598790$ \\
\hline 4.65 & 0.10 & 1.99 & 0.01 & 197.9350 & 7.469130 \\
\hline 4.65 & 0.10 & 2.00 & 0.01 & 199.6390 & $7 \cdot 564340$ \\
\hline 4.65 & 0.10 & 2.01 & 0.01 & 209.7960 & $7 \cdot 749920$ \\
\hline 4.65 & 0.10 & 2.02 & 0.01 & 222.8210 & 8.058960 \\
\hline 4.65 & 0.10 & 2.03 & 0.01 & 209.1690 & 7.629160 \\
\hline 4.65 & 0.10 & 2.04 & 0.01 & $217 \cdot 5780$ & $7 \cdot 792400$ \\
\hline 4.65 & 0.10 & 2.05 & 0.01 & 213.4520 & 7.639080 \\
\hline 4.65 & 0.10 & 2.06 & 0.01 & $215 \cdot 5610$ & 7.837920 \\
\hline 4.65 & 0.10 & 2.07 & 0.01 & 203.1320 & $7 \cdot 536950$ \\
\hline 4.65 & 0.10 & 2.08 & 0.01 & 200.4530 & $7 \cdot 360310$ \\
\hline 4.65 & 0.10 & 2.09 & 0.01 & 193.4880 & $7 \cdot 337440$ \\
\hline 4.65 & 0.10 & 2.10 & 0.01 & 176.9380 & 7.029140 \\
\hline 4.65 & 0.10 & 2.11 & 0.01 & 193.1580 & $7 \cdot 735310$ \\
\hline 4.65 & 0.10 & 2.12 & 0.01 & 185.1200 & $7 \cdot 511260$ \\
\hline 4.65 & 0.10 & 2.13 & 0.01 & 162.4130 & 7.025980 \\
\hline 4.65 & 0.10 & 2.14 & 0.01 & $145 \cdot 4110$ & 6.583090 \\
\hline 4.65 & 0.10 & 2.15 & 0.01 & 141.9230 & 6.588220 \\
\hline 4.65 & 0.10 & 2.16 & 0.01 & $124 \cdot 3820$ & 6.433770 \\
\hline 4.65 & 0.10 & 2.17 & 0.01 & 102.5800 & 5.909090 \\
\hline
\end{tabular}


Table A.4 - Continued

\begin{tabular}{|c|c|c|c|c|c|}
\hline$E_{\text {beam }}^{\text {lab }}[\mathrm{GeV}]$ & $\pm d E_{\text {beam }}^{\text {lab }}$ & $M(p \bar{p})\left[\frac{G e V}{c^{2}}\right]$ & $\pm d M(p \bar{p})$ & $\frac{d \sigma}{d M(p \bar{p})}[n b]$ & $\sigma^{\text {stat }}$ \\
\hline 4.65 & 0.10 & 2.18 & 0.01 & 94.2126 & 6.131540 \\
\hline 4.65 & 0.10 & 2.19 & 0.01 & 72.3305 & 5.711760 \\
\hline 4.65 & 0.10 & 2.20 & 0.01 & 39.3966 & 5.112050 \\
\hline 4.65 & 0.10 & 2.21 & 0.01 & 20.6514 & 4.467320 \\
\hline $4 \cdot 75$ & 0.10 & 1.92 & 0.01 & 24.0653 & 2.559020 \\
\hline $4 \cdot 75$ & 0.10 & 1.93 & 0.01 & 84.9166 & 5.331740 \\
\hline 4.75 & 0.10 & 1.94 & 0.01 & 133.9510 & 6.724260 \\
\hline $4 \cdot 75$ & 0.10 & 1.95 & 0.01 & $167 \cdot 5880$ & 7.923690 \\
\hline $4 \cdot 75$ & 0.10 & 1.96 & 0.01 & 174.8050 & 7.832800 \\
\hline $4 \cdot 75$ & 0.10 & 1.97 & 0.01 & 200.1050 & 8.411560 \\
\hline $4 \cdot 75$ & 0.10 & 1.98 & 0.01 & 221.7850 & 9.073320 \\
\hline $4 \cdot 75$ & 0.10 & 1.99 & 0.01 & $225 \cdot 5230$ & 9.006630 \\
\hline 4.75 & 0.10 & 2.00 & 0.01 & 235.6310 & 9.240560 \\
\hline $4 \cdot 75$ & 0.10 & 2.01 & 0.01 & 228.2900 & 9.004300 \\
\hline 4.75 & 0.10 & 2.02 & 0.01 & 230.0410 & 8.772440 \\
\hline 4.75 & 0.10 & 2.03 & 0.01 & 246.2640 & $9 \cdot 398770$ \\
\hline 4.75 & 0.10 & 2.04 & 0.01 & 251.0630 & $9 \cdot 411180$ \\
\hline 4.75 & 0.10 & 2.05 & 0.01 & 243.1050 & 8.933670 \\
\hline 4.75 & 0.10 & 2.06 & 0.01 & 257.8260 & $9 \cdot 503470$ \\
\hline $4 \cdot 75$ & 0.10 & 2.07 & 0.01 & 238.0100 & 8.958760 \\
\hline $4 \cdot 75$ & 0.10 & 2.08 & 0.01 & 230.1460 & 8.784640 \\
\hline 4.75 & 0.10 & 2.09 & 0.01 & 245.1060 & 9.215030 \\
\hline $4 \cdot 75$ & 0.10 & 2.10 & 0.01 & 220.0180 & 8.520610 \\
\hline 4.75 & 0.10 & 2.11 & 0.01 & 224.1540 & 8.801650 \\
\hline
\end{tabular}


Table A.4 - Continued

\begin{tabular}{|c|c|c|c|c|c|}
\hline$E_{\text {beam }}^{\text {lab }}[\mathrm{GeV}]$ & $\pm d E_{\text {beam }}^{\text {lab }}$ & $M(p \bar{p})\left[\frac{G e V}{c^{2}}\right]$ & $\pm d M(p \bar{p})$ & $\frac{d \sigma}{d M(p \bar{p})}[n b]$ & $\sigma^{\text {stat }}$ \\
\hline 4.75 & 0.10 & 2.12 & 0.01 & 222.0350 & 8.960770 \\
\hline $4 \cdot 75$ & 0.10 & 2.13 & 0.01 & 192.1420 & 8.135030 \\
\hline $4 \cdot 75$ & 0.10 & 2.14 & 0.01 & 188.9540 & 8.068220 \\
\hline 4.75 & 0.10 & 2.15 & 0.01 & 187.7480 & 8.120270 \\
\hline 4.75 & 0.10 & 2.16 & 0.01 & 175.7930 & 8.023570 \\
\hline $4 \cdot 75$ & 0.10 & 2.17 & 0.01 & 161.2050 & $7 \cdot 760580$ \\
\hline $4 \cdot 75$ & 0.10 & 2.18 & 0.01 & 159.9260 & 7.992580 \\
\hline $4 \cdot 75$ & 0.10 & 2.19 & 0.01 & 131.1900 & 7.205520 \\
\hline 4.75 & 0.10 & 2.20 & 0.01 & 126.8710 & $7 \cdot 729620$ \\
\hline $4 \cdot 75$ & 0.10 & 2.21 & 0.01 & 108.5080 & $7 \cdot 356020$ \\
\hline $4 \cdot 75$ & 0.10 & 2.22 & 0.01 & 70.2459 & 6.343490 \\
\hline $4 \cdot 75$ & 0.10 & 2.23 & 0.01 & 56.0572 & 7.223620 \\
\hline 4.75 & 0.10 & 2.24 & 0.01 & 36.3413 & 7.515600 \\
\hline 4.85 & 0.10 & 1.92 & 0.01 & 23.3697 & 2.215020 \\
\hline 4.85 & 0.10 & 1.93 & 0.01 & 75.6996 & 4.650130 \\
\hline 4.85 & 0.10 & 1.94 & 0.01 & 103.0200 & $5 \cdot 304070$ \\
\hline 4.85 & 0.10 & 1.95 & 0.01 & 153.5520 & 6.718770 \\
\hline 4.85 & 0.10 & 1.96 & 0.01 & 163.4060 & 6.793400 \\
\hline 4.85 & 0.10 & 1.97 & 0.01 & 171.9220 & 6.918580 \\
\hline 4.85 & 0.10 & 1.98 & 0.01 & 196.7220 & $7 \cdot 385810$ \\
\hline 4.85 & 0.10 & 1.99 & 0.01 & 212.3560 & 7.616480 \\
\hline 4.85 & 0.10 & 2.00 & 0.01 & 216.1110 & 7.658420 \\
\hline 4.85 & 0.10 & 2.01 & 0.01 & 201.1170 & 7.197640 \\
\hline 4.85 & 0.10 & 2.02 & 0.01 & 200.9370 & 7.210110 \\
\hline
\end{tabular}


Table A.4 - Continued

\begin{tabular}{|c|c|c|c|c|c|}
\hline$E_{\text {beam }}^{\text {lab }}[\mathrm{GeV}]$ & $\pm d E_{\text {beam }}^{\text {lab }}$ & $M(p \bar{p})\left[\frac{G e V}{c^{2}}\right]$ & $\pm d M(p \bar{p})$ & $\frac{d \sigma}{d M(p \bar{p})}[n b]$ & $\sigma^{\text {stat }}$ \\
\hline 4.85 & 0.10 & 2.03 & 0.01 & 218.6040 & $7 \cdot 700930$ \\
\hline 4.85 & 0.10 & 2.04 & 0.01 & 220.6150 & 7.556320 \\
\hline 4.85 & 0.10 & 2.05 & 0.01 & 216.9270 & $7 \cdot 392860$ \\
\hline 4.85 & 0.10 & 2.06 & 0.01 & 236.9970 & 7.934670 \\
\hline 4.85 & 0.10 & 2.07 & 0.01 & 225.1470 & $7 \cdot 720390$ \\
\hline 4.85 & 0.10 & 2.08 & 0.01 & 214.1530 & 7.291580 \\
\hline 4.85 & 0.10 & 2.09 & 0.01 & 221.1060 & 7.685970 \\
\hline 4.85 & 0.10 & 2.10 & 0.01 & 210.1710 & 7.258660 \\
\hline 4.85 & 0.10 & 2.11 & 0.01 & 209.9390 & $7 \cdot 360980$ \\
\hline 4.85 & 0.10 & 2.12 & 0.01 & 198.6150 & 7.085020 \\
\hline 4.85 & 0.10 & 2.13 & 0.01 & 195.9950 & 7.160020 \\
\hline 4.85 & 0.10 & 2.14 & 0.01 & 178.4940 & 6.647420 \\
\hline 4.85 & 0.10 & 2.15 & 0.01 & 181.7180 & 6.957410 \\
\hline 4.85 & 0.10 & 2.16 & 0.01 & 169.3230 & 6.657560 \\
\hline 4.85 & 0.10 & 2.17 & 0.01 & 177.9560 & 7.122160 \\
\hline 4.85 & 0.10 & 2.18 & 0.01 & 164.2850 & 6.817430 \\
\hline 4.85 & 0.10 & 2.19 & 0.01 & 148.2060 & 6.461510 \\
\hline 4.85 & 0.10 & 2.20 & 0.01 & 146.0060 & 6.392290 \\
\hline 4.85 & 0.10 & 2.21 & 0.01 & 134.2460 & 6.405030 \\
\hline 4.85 & 0.10 & 2.22 & 0.01 & 115.6010 & 6.066800 \\
\hline 4.85 & 0.10 & 2.23 & 0.01 & 88.4773 & 5.401280 \\
\hline 4.85 & 0.10 & 2.24 & 0.01 & 73.4459 & 5.324740 \\
\hline 4.85 & 0.10 & 2.25 & 0.01 & 60.8842 & 5.123200 \\
\hline 4.85 & 0.10 & 2.26 & 0.01 & 36.4396 & $4 \cdot 512760$ \\
\hline
\end{tabular}


Table A.4 - Continued

\begin{tabular}{|c|c|c|c|c|c|}
\hline$E_{\text {beam }}^{\text {lab }}[\mathrm{GeV}]$ & $\pm d E_{\text {beam }}^{\text {lab }}$ & $M(p \bar{p})\left[\frac{G e V}{c^{2}}\right]$ & $\pm d M(p \bar{p})$ & $\frac{d \sigma}{d M(p \bar{p})}[n b]$ & $\sigma^{\text {stat }}$ \\
\hline 4.85 & 0.10 & 2.27 & 0.01 & 22.3953 & 4.054620 \\
\hline 4.85 & 0.10 & 2.28 & 0.01 & 3.8517 & 3.883580 \\
\hline 4.95 & 0.10 & 1.92 & 0.01 & 18.7736 & 1.945680 \\
\hline 4.95 & 0.10 & 1.93 & 0.01 & 87.6234 & $5 \cdot 540160$ \\
\hline 4.95 & 0.10 & 1.94 & 0.01 & 114.8770 & 6.253930 \\
\hline 4.95 & 0.10 & 1.95 & 0.01 & 157.4160 & $7 \cdot 530470$ \\
\hline 4.95 & 0.10 & 1.96 & 0.01 & 169.6100 & 7.660850 \\
\hline 4.95 & 0.10 & 1.97 & 0.01 & 200.3370 & 8.816860 \\
\hline 4.95 & 0.10 & 1.98 & 0.01 & 199.6150 & 8.323680 \\
\hline 4.95 & 0.10 & 1.99 & 0.01 & 198.2440 & 8.121450 \\
\hline 4.95 & 0.10 & 2.00 & 0.01 & 217.2880 & 8.700950 \\
\hline 4.95 & 0.10 & 2.01 & 0.01 & 241.6070 & 9.065480 \\
\hline 4.95 & 0.10 & 2.02 & 0.01 & 236.3220 & 8.908310 \\
\hline 4.95 & 0.10 & 2.03 & 0.01 & 242.7910 & 9.160320 \\
\hline 4.95 & 0.10 & 2.04 & 0.01 & $245 \cdot 7040$ & 9.089390 \\
\hline 4.95 & 0.10 & 2.05 & 0.01 & 237.4910 & 8.694700 \\
\hline 4.95 & 0.10 & 2.06 & 0.01 & 230.9790 & 8.612980 \\
\hline 4.95 & 0.10 & 2.07 & 0.01 & 260.6900 & 9.479310 \\
\hline 4.95 & 0.10 & 2.08 & 0.01 & 244.4350 & 8.939970 \\
\hline 4.95 & 0.10 & 2.09 & 0.01 & 249.5350 & 9.178670 \\
\hline 4.95 & 0.10 & 2.10 & 0.01 & 242.1080 & 9.063450 \\
\hline 4.95 & 0.10 & 2.11 & 0.01 & 235.1280 & 8.861790 \\
\hline 4.95 & 0.10 & 2.12 & 0.01 & 227.8190 & 8.571110 \\
\hline 4.95 & 0.10 & 2.13 & 0.01 & $217 \cdot 4770$ & 8.477290 \\
\hline
\end{tabular}


Table A.4 - Continued

\begin{tabular}{|c|c|c|c|c|c|}
\hline$E_{\text {beam }}^{l a b}[\mathrm{GeV}]$ & $\pm d E_{\text {beam }}^{l a b}$ & $M(p \bar{p})\left[\frac{G e V}{c^{2}}\right]$ & $\pm d M(p \bar{p})$ & $\frac{d \sigma}{d M(p \bar{p})}[n b]$ & $\sigma^{\text {stat }}$ \\
\hline 4.95 & 0.10 & 2.14 & 0.01 & 215.8890 & 8.373160 \\
\hline 4.95 & 0.10 & 2.15 & 0.01 & $207 \cdot 4730$ & 8.133820 \\
\hline 4.95 & 0.10 & 2.16 & 0.01 & 212.1940 & 8.483010 \\
\hline 4.95 & 0.10 & 2.17 & 0.01 & 188.8180 & $7 \cdot 771430$ \\
\hline 4.95 & 0.10 & 2.18 & 0.01 & 186.4850 & 7.805190 \\
\hline 4.95 & 0.10 & 2.19 & 0.01 & 203.0850 & 8.557280 \\
\hline 4.95 & 0.10 & 2.20 & 0.01 & 179.7190 & 8.001900 \\
\hline 4.95 & 0.10 & 2.21 & 0.01 & 160.3870 & $7 \cdot 368130$ \\
\hline 4.95 & 0.10 & 2.22 & 0.01 & 139.6700 & 6.866830 \\
\hline 4.95 & 0.10 & 2.23 & 0.01 & 156.2210 & $7 \cdot 501150$ \\
\hline 4.95 & 0.10 & 2.24 & 0.01 & 126.2780 & 6.643600 \\
\hline 4.95 & 0.10 & 2.25 & 0.01 & 108.5740 & 6.427200 \\
\hline 4.95 & 0.10 & 2.26 & 0.01 & 104.9650 & 6.828670 \\
\hline 4.95 & 0.10 & 2.27 & 0.01 & $74 \cdot 4987$ & $5 \cdot 762040$ \\
\hline 4.95 & 0.10 & 2.28 & 0.01 & 61.3432 & 5.999090 \\
\hline 4.95 & 0.10 & 2.29 & 0.01 & $39 \cdot 5336$ & 5.498380 \\
\hline 4.95 & 0.10 & 2.30 & 0.01 & 8.2526 & $3.335^{120}$ \\
\hline 4.95 & 0.10 & 2.31 & 0.01 & 6.2666 & 6.338240 \\
\hline 5.05 & 0.10 & 1.92 & 0.01 & 17.6962 & 1.714200 \\
\hline 5.05 & 0.10 & 1.93 & 0.01 & $74 \cdot 5707$ & 4.682060 \\
\hline 5.05 & 0.10 & 1.94 & 0.01 & $107 \cdot 5510$ & 5.642300 \\
\hline 5.05 & 0.10 & 1.95 & 0.01 & 130.4950 & 6.136420 \\
\hline 5.05 & 0.10 & 1.96 & 0.01 & 151.5330 & 6.792090 \\
\hline 5.05 & 0.10 & 1.97 & 0.01 & 176.6110 & 7.129930 \\
\hline
\end{tabular}


Table A.4 - Continued

\begin{tabular}{|c|c|c|c|c|c|}
\hline$E_{\text {beam }}^{l a b}[\mathrm{GeV}]$ & $\pm d E_{\text {beam }}^{\text {lab }}$ & $M(p \bar{p})\left[\frac{G e V}{c^{2}}\right]$ & $\pm d M(p \bar{p})$ & $\frac{d \sigma}{d M(p \bar{p})}[n b]$ & $\sigma^{\text {stat }}$ \\
\hline 5.05 & 0.10 & 1.98 & 0.01 & 173.4980 & 6.932140 \\
\hline 5.05 & 0.10 & 1.99 & 0.01 & 180.3650 & 6.992450 \\
\hline 5.05 & 0.10 & 2.00 & 0.01 & 212.1410 & 7.893350 \\
\hline 5.05 & 0.10 & 2.01 & 0.01 & 210.7220 & 7.664500 \\
\hline 5.05 & 0.10 & 2.02 & 0.01 & 222.7420 & 7.877430 \\
\hline 5.05 & 0.10 & 2.03 & 0.01 & $235 \cdot 5710$ & 8.111010 \\
\hline 5.05 & 0.10 & 2.04 & 0.01 & 233.8290 & 8.126190 \\
\hline 5.05 & 0.10 & 2.05 & 0.01 & 233.9160 & 8.006170 \\
\hline 5.05 & 0.10 & 2.06 & 0.01 & 230.6430 & 7.906710 \\
\hline 5.05 & 0.10 & 2.07 & 0.01 & $243 \cdot 5830$ & 8.232370 \\
\hline 5.05 & 0.10 & 2.08 & 0.01 & $235 \cdot 5730$ & 8.105650 \\
\hline 5.05 & 0.10 & 2.09 & 0.01 & 235.8420 & 8.030970 \\
\hline 5.05 & 0.10 & 2.10 & 0.01 & 226.0760 & 7.682450 \\
\hline 5.05 & 0.10 & 2.11 & 0.01 & 208.4520 & 7.231410 \\
\hline 5.05 & 0.10 & 2.12 & 0.01 & 210.2330 & $7 \cdot 342600$ \\
\hline 5.05 & 0.10 & 2.13 & 0.01 & 222.2360 & $7 \cdot 719400$ \\
\hline 5.05 & 0.10 & 2.14 & 0.01 & 201.7860 & 7.271210 \\
\hline 5.05 & 0.10 & 2.15 & 0.01 & $194 \cdot 3090$ & 7.005360 \\
\hline 5.05 & 0.10 & 2.16 & 0.01 & 210.3700 & $7 \cdot 598340$ \\
\hline 5.05 & 0.10 & 2.17 & 0.01 & 216.1110 & $7 \cdot 730200$ \\
\hline 5.05 & 0.10 & 2.18 & 0.01 & 197.1730 & 7.276690 \\
\hline 5.05 & 0.10 & 2.19 & 0.01 & 188.4000 & 7.162800 \\
\hline 5.05 & 0.10 & 2.20 & 0.01 & 172.3750 & 6.875220 \\
\hline 5.05 & 0.10 & 2.21 & 0.01 & 169.1650 & 6.877270 \\
\hline
\end{tabular}


Table A.4 - Continued

\begin{tabular}{|c|c|c|c|c|c|}
\hline$E_{\text {beam }}^{\text {lab }}[\mathrm{GeV}]$ & $\pm d E_{\text {beam }}^{\text {lab }}$ & $M(p \bar{p})\left[\frac{G e V}{c^{2}}\right]$ & $\pm d M(p \bar{p})$ & $\frac{d \sigma}{d M(p \bar{p})}[n b]$ & $\sigma^{\text {stat }}$ \\
\hline 5.05 & 0.10 & 2.22 & 0.01 & 164.3920 & 6.873610 \\
\hline 5.05 & 0.10 & 2.23 & 0.01 & 160.0380 & 6.799870 \\
\hline 5.05 & 0.10 & 2.24 & 0.01 & 143.5240 & 6.313090 \\
\hline 5.05 & 0.10 & 2.25 & 0.01 & 140.3280 & 6.299440 \\
\hline 5.05 & 0.10 & 2.26 & 0.01 & 107.7090 & $5 \cdot 312360$ \\
\hline 5.05 & 0.10 & 2.27 & 0.01 & 116.9460 & 6.027730 \\
\hline 5.05 & 0.10 & 2.28 & 0.01 & 95.9881 & 5.570870 \\
\hline 5.05 & 0.10 & 2.29 & 0.01 & 79.9289 & 5.339730 \\
\hline 5.05 & 0.10 & 2.30 & 0.01 & 61.0094 & 5.064360 \\
\hline 5.05 & 0.10 & 2.31 & 0.01 & 52.6975 & 5.438000 \\
\hline 5.05 & 0.10 & 2.32 & 0.01 & 30.7785 & 4.269260 \\
\hline 5.05 & 0.10 & 2.33 & 0.01 & 16.6590 & 4.287150 \\
\hline 5.15 & 0.10 & 1.92 & 0.01 & 20.9934 & 2.105920 \\
\hline 5.15 & 0.10 & 1.93 & 0.01 & 84.8648 & 5.399890 \\
\hline 5.15 & 0.10 & 1.94 & 0.01 & 114.7070 & 6.203730 \\
\hline 5.15 & 0.10 & 1.95 & 0.01 & 137.1350 & 6.744850 \\
\hline 5.15 & 0.10 & 1.96 & 0.01 & 148.7110 & 6.920230 \\
\hline 5.15 & 0.10 & 1.97 & 0.01 & 171.9930 & $7 \cdot 599180$ \\
\hline 5.15 & 0.10 & 1.98 & 0.01 & 185.1430 & 7.716310 \\
\hline 5.15 & 0.10 & 1.99 & 0.01 & 189.2860 & $7 \cdot 753790$ \\
\hline 5.15 & 0.10 & 2.00 & 0.01 & 205.8690 & 8.118970 \\
\hline 5.15 & 0.10 & 2.01 & 0.01 & 232.2440 & 8.668690 \\
\hline 5.15 & 0.10 & 2.02 & 0.01 & 206.1380 & 7.946240 \\
\hline 5.15 & 0.10 & 2.03 & 0.01 & 222.7750 & 8.330990 \\
\hline
\end{tabular}


Table A.4 - Continued

\begin{tabular}{|c|c|c|c|c|c|}
\hline$E_{\text {beam }}^{l a b}[\mathrm{GeV}]$ & $\pm d E_{\text {beam }}^{\text {lab }}$ & $M(p \bar{p})\left[\frac{G e V}{c^{2}}\right]$ & $\pm d M(p \bar{p})$ & $\frac{d \sigma}{d M(p \bar{p})}[n b]$ & $\sigma^{\text {stat }}$ \\
\hline 5.15 & 0.10 & 2.04 & 0.01 & 219.8700 & 8.164750 \\
\hline 5.15 & 0.10 & 2.05 & 0.01 & 227.7310 & 8.409230 \\
\hline 5.15 & 0.10 & 2.06 & 0.01 & 232.5120 & 8.537960 \\
\hline 5.15 & 0.10 & 2.07 & 0.01 & 230.7190 & 8.422100 \\
\hline 5.15 & 0.10 & 2.08 & 0.01 & $227 \cdot 7660$ & 8.241820 \\
\hline 5.15 & 0.10 & 2.09 & 0.01 & 222.5960 & 8.024160 \\
\hline 5.15 & 0.10 & 2.10 & 0.01 & 224.8680 & 8.175530 \\
\hline 5.15 & 0.10 & 2.11 & 0.01 & 241.9260 & 8.733820 \\
\hline 5.15 & 0.10 & 2.12 & 0.01 & 232.0030 & 8.351540 \\
\hline 5.15 & 0.10 & 2.13 & 0.01 & 215.8880 & 7.954870 \\
\hline 5.15 & 0.10 & 2.14 & 0.01 & 216.7790 & 8.082630 \\
\hline 5.15 & 0.10 & 2.15 & 0.01 & 239.1260 & 8.636300 \\
\hline 5.15 & 0.10 & 2.16 & 0.01 & 207.1700 & 7.800140 \\
\hline 5.15 & 0.10 & 2.17 & 0.01 & 214.4370 & 8.113780 \\
\hline 5.15 & 0.10 & 2.18 & 0.01 & 207.6500 & 7.973340 \\
\hline 5.15 & 0.10 & 2.19 & 0.01 & $197 \cdot 3940$ & $7 \cdot 764390$ \\
\hline 5.15 & 0.10 & 2.20 & 0.01 & 208.0710 & 8.06906o \\
\hline 5.15 & 0.10 & 2.21 & 0.01 & $173 \cdot 3440$ & 7.068790 \\
\hline 5.15 & 0.10 & 2.22 & 0.01 & 174.2700 & 7.219140 \\
\hline 5.15 & 0.10 & 2.23 & 0.01 & 158.7890 & 6.949470 \\
\hline 5.15 & 0.10 & 2.24 & 0.01 & 162.2010 & 6.919740 \\
\hline 5.15 & 0.10 & 2.25 & 0.01 & 156.7930 & 7.008410 \\
\hline 5.15 & 0.10 & 2.26 & 0.01 & 153.0610 & 6.963710 \\
\hline 5.15 & 0.10 & 2.27 & 0.01 & 142.3900 & 6.824290 \\
\hline
\end{tabular}


Table A.4 - Continued

\begin{tabular}{|c|c|c|c|c|c|}
\hline$E_{\text {beam }}^{\text {lab }}[\mathrm{GeV}]$ & $\pm d E_{\text {beam }}^{\text {lab }}$ & $M(p \bar{p})\left[\frac{G e V}{c^{2}}\right]$ & $\pm d M(p \bar{p})$ & $\frac{d \sigma}{d M(p \bar{p})}[n b]$ & $\sigma^{\text {stat }}$ \\
\hline 5.15 & 0.10 & 2.28 & 0.01 & 126.9270 & 6.279580 \\
\hline 5.15 & 0.10 & 2.29 & 0.01 & 115.6030 & 6.031400 \\
\hline 5.15 & 0.10 & 2.30 & 0.01 & 108.4080 & 6.027060 \\
\hline 5.15 & 0.10 & 2.31 & 0.01 & 87.8702 & 5.737560 \\
\hline 5.15 & 0.10 & 2.32 & 0.01 & 70.3238 & 5.186560 \\
\hline 5.15 & 0.10 & 2.33 & 0.01 & 62.6028 & 5.296410 \\
\hline 5.15 & 0.10 & 2.34 & 0.01 & 44.3844 & 4.776810 \\
\hline 5.15 & 0.10 & 2.35 & 0.01 & 34.0097 & 5.844330 \\
\hline 5.15 & 0.10 & 2.36 & 0.01 & 5.0095 & 1.701260 \\
\hline 5.25 & 0.10 & 1.92 & 0.01 & 18.1709 & 1.816400 \\
\hline 5.25 & 0.10 & 1.93 & 0.01 & 72.3427 & 5.034550 \\
\hline 5.25 & 0.10 & 1.94 & 0.01 & 98.6058 & 5.901920 \\
\hline 5.25 & 0.10 & 1.95 & 0.01 & 120.2310 & 6.491700 \\
\hline 5.25 & 0.10 & 1.96 & 0.01 & 150.7970 & $7 \cdot 396360$ \\
\hline 5.25 & 0.10 & 1.97 & 0.01 & 156.1340 & $7 \cdot 46208 \mathrm{o}$ \\
\hline 5.25 & 0.10 & 1.98 & 0.01 & 176.2400 & 7.993460 \\
\hline 5.25 & 0.10 & 1.99 & 0.01 & 192.7270 & 8.433060 \\
\hline 5.25 & 0.10 & 2.00 & 0.01 & 183.0790 & 7.938600 \\
\hline 5.25 & 0.10 & 2.01 & 0.01 & 210.0260 & 8.579830 \\
\hline 5.25 & 0.10 & 2.02 & 0.01 & 205.0750 & 8.430290 \\
\hline 5.25 & 0.10 & 2.03 & 0.01 & 215.4130 & 8.804930 \\
\hline 5.25 & 0.10 & 2.04 & 0.01 & 212.6440 & 8.572990 \\
\hline 5.25 & 0.10 & 2.05 & 0.01 & 209.7150 & 8.284140 \\
\hline 5.25 & 0.10 & 2.06 & 0.01 & 224.1960 & 8.774190 \\
\hline
\end{tabular}


Table A.4 - Continued

\begin{tabular}{|c|c|c|c|c|c|}
\hline$E_{\text {beam }}^{\text {lab }}[\mathrm{GeV}]$ & $\pm d E_{\text {beam }}^{\text {lab }}$ & $M(p \bar{p})\left[\frac{G e V}{c^{2}}\right]$ & $\pm d M(p \bar{p})$ & $\frac{d \sigma}{d M(p \bar{p})}[n b]$ & $\sigma^{\text {stat }}$ \\
\hline 5.25 & 0.10 & 2.07 & 0.01 & 219.1080 & 8.453950 \\
\hline 5.25 & 0.10 & 2.08 & 0.01 & 214.3910 & 8.363610 \\
\hline 5.25 & 0.10 & 2.09 & 0.01 & 221.3410 & 8.739250 \\
\hline 5.25 & 0.10 & 2.10 & 0.01 & 222.8760 & 8.648090 \\
\hline 5.25 & 0.10 & 2.11 & 0.01 & 217.0250 & 8.403790 \\
\hline 5.25 & 0.10 & 2.12 & 0.01 & 233.7560 & 8.950310 \\
\hline 5.25 & 0.10 & 2.13 & 0.01 & 224.5880 & 8.492460 \\
\hline 5.25 & 0.10 & 2.14 & 0.01 & 209.9120 & 8.226770 \\
\hline 5.25 & 0.10 & 2.15 & 0.01 & 213.1960 & 8.309710 \\
\hline 5.25 & 0.10 & 2.16 & 0.01 & 213.5440 & 8.295120 \\
\hline 5.25 & 0.10 & 2.17 & 0.01 & 211.5340 & 8.360690 \\
\hline 5.25 & 0.10 & 2.18 & 0.01 & 213.4630 & 8.457770 \\
\hline 5.25 & 0.10 & 2.19 & 0.01 & 198.3490 & 8.008210 \\
\hline 5.25 & 0.10 & 2.20 & 0.01 & 189.0530 & $7 \cdot 706650$ \\
\hline 5.25 & 0.10 & 2.21 & 0.01 & 184.0910 & $7 \cdot 787290$ \\
\hline 5.25 & 0.10 & 2.22 & 0.01 & 189.8050 & 8.053890 \\
\hline 5.25 & 0.10 & 2.23 & 0.01 & 186.5580 & 7.921490 \\
\hline 5.25 & 0.10 & 2.24 & 0.01 & 174.9660 & $7 \cdot 582920$ \\
\hline 5.25 & 0.10 & 2.25 & 0.01 & 169.5380 & 7.585510 \\
\hline 5.25 & 0.10 & 2.26 & 0.01 & 156.2780 & 7.166840 \\
\hline 5.25 & 0.10 & 2.27 & 0.01 & 144.2070 & 6.803100 \\
\hline 5.25 & 0.10 & 2.28 & 0.01 & 136.9320 & 6.656000 \\
\hline 5.25 & 0.10 & 2.29 & 0.01 & 137.1760 & 6.665690 \\
\hline 5.25 & 0.10 & 2.30 & 0.01 & 127.0990 & 6.538360 \\
\hline
\end{tabular}


Table A.4 - Continued

\begin{tabular}{|c|c|c|c|c|c|}
\hline$E_{\text {beam }}^{\text {lab }}[\mathrm{GeV}]$ & $\pm d E_{\text {beam }}^{\text {lab }}$ & $M(p \bar{p})\left[\frac{G e V}{c^{2}}\right]$ & $\pm d M(p \bar{p})$ & $\frac{d \sigma}{d M(p \bar{p})}[n b]$ & $\sigma^{\text {stat }}$ \\
\hline 5.25 & 0.10 & 2.31 & 0.01 & 100.1270 & 5.607440 \\
\hline 5.25 & 0.10 & 2.32 & 0.01 & 97.7982 & 5.941480 \\
\hline 5.25 & 0.10 & 2.33 & 0.01 & 97.2784 & 5.909190 \\
\hline 5.25 & 0.10 & 2.34 & 0.01 & 96.7387 & 6.447660 \\
\hline 5.25 & 0.10 & 2.35 & 0.01 & 78.4963 & 5.989700 \\
\hline 5.25 & 0.10 & 2.36 & 0.01 & 45.2896 & 4.892530 \\
\hline 5.25 & 0.10 & 2.37 & 0.01 & 26.2508 & 4.140800 \\
\hline 5.25 & 0.10 & 2.38 & 0.01 & 26.5193 & 4.974400 \\
\hline 5.25 & 0.10 & 2.39 & 0.01 & 2.2492 & 2.255220 \\
\hline $5 \cdot 35$ & 0.10 & 1.92 & 0.01 & 19.2762 & 2.006720 \\
\hline 5.35 & 0.10 & 1.93 & 0.01 & 71.0753 & 4.937860 \\
\hline $5 \cdot 35$ & 0.10 & 1.94 & 0.01 & 112.7780 & 6.102930 \\
\hline $5 \cdot 35$ & 0.10 & 1.95 & 0.01 & 128.2270 & 6.403450 \\
\hline $5 \cdot 35$ & 0.10 & 1.96 & 0.01 & 145.7730 & 7.007380 \\
\hline $5 \cdot 35$ & 0.10 & 1.97 & 0.01 & 156.3670 & 7.050810 \\
\hline $5 \cdot 35$ & 0.10 & 1.98 & 0.01 & 182.0930 & 7.863070 \\
\hline $5 \cdot 35$ & 0.10 & 1.99 & 0.01 & 178.2020 & 7.465290 \\
\hline $5 \cdot 35$ & 0.10 & 2.00 & 0.01 & 189.1630 & 7.627050 \\
\hline $5 \cdot 35$ & 0.10 & 2.01 & 0.01 & 211.8530 & 8.365670 \\
\hline $5 \cdot 35$ & 0.10 & 2.02 & 0.01 & 204.9320 & 8.112060 \\
\hline $5 \cdot 35$ & 0.10 & 2.03 & 0.01 & 220.9260 & 8.477190 \\
\hline $5 \cdot 35$ & 0.10 & 2.04 & 0.01 & 221.8260 & 8.427120 \\
\hline $5 \cdot 35$ & 0.10 & 2.05 & 0.01 & 228.0440 & 8.649510 \\
\hline 5.35 & 0.10 & 2.06 & 0.01 & 229.8720 & $8.64288 \mathrm{o}$ \\
\hline
\end{tabular}


Table A.4 - Continued

\begin{tabular}{|c|c|c|c|c|c|}
\hline$E_{\text {beam }}^{\text {lab }}[\mathrm{GeV}]$ & $\pm d E_{\text {beam }}^{\text {lab }}$ & $M(p \bar{p})\left[\frac{G e V}{c^{2}}\right]$ & $\pm d M(p \bar{p})$ & $\frac{d \sigma}{d M(p \bar{p})}[n b]$ & $\sigma^{\text {stat }}$ \\
\hline $5 \cdot 35$ & 0.10 & 2.07 & 0.01 & 209.5440 & $7.86978 \mathrm{o}$ \\
\hline 5.35 & 0.10 & 2.08 & 0.01 & 213.4490 & 8.046430 \\
\hline $5 \cdot 35$ & 0.10 & 2.09 & 0.01 & 225.0390 & 8.309090 \\
\hline 5.35 & 0.10 & 2.10 & 0.01 & 240.8620 & 8.646010 \\
\hline $5 \cdot 35$ & 0.10 & 2.11 & 0.01 & $229 \cdot 3000$ & 8.472420 \\
\hline $5 \cdot 35$ & 0.10 & 2.12 & 0.01 & 221.0330 & 8.109380 \\
\hline $5 \cdot 35$ & 0.10 & 2.13 & 0.01 & 246.1790 & 8.688650 \\
\hline $5 \cdot 35$ & 0.10 & 2.14 & 0.01 & 236.3940 & 8.534860 \\
\hline 5.35 & 0.10 & 2.15 & 0.01 & 240.7790 & 8.734630 \\
\hline $5 \cdot 35$ & 0.10 & 2.16 & 0.01 & 214.1450 & 8.011160 \\
\hline 5.35 & 0.10 & 2.17 & 0.01 & 222.3380 & 8.195910 \\
\hline $5 \cdot 35$ & 0.10 & 2.18 & 0.01 & 215.0370 & 8.069010 \\
\hline $5 \cdot 35$ & 0.10 & 2.19 & 0.01 & 213.8820 & 8.032070 \\
\hline $5 \cdot 35$ & 0.10 & 2.20 & 0.01 & 201.1620 & 7.697890 \\
\hline $5 \cdot 35$ & 0.10 & 2.21 & 0.01 & 210.9440 & 8.158660 \\
\hline $5 \cdot 35$ & 0.10 & 2.22 & 0.01 & 199.9750 & 7.801330 \\
\hline $5 \cdot 35$ & 0.10 & 2.23 & 0.01 & 184.9290 & $7 \cdot 317730$ \\
\hline $5 \cdot 35$ & 0.10 & 2.24 & 0.01 & 187.1470 & $7 \cdot 490830$ \\
\hline $5 \cdot 35$ & 0.10 & 2.25 & 0.01 & 172.2470 & 7.087040 \\
\hline $5 \cdot 35$ & 0.10 & 2.26 & 0.01 & 160.4310 & 7.002270 \\
\hline $5 \cdot 35$ & 0.10 & 2.27 & 0.01 & 168.3240 & 7.214440 \\
\hline $5 \cdot 35$ & 0.10 & 2.28 & 0.01 & 170.7340 & $7 \cdot 419980$ \\
\hline $5 \cdot 35$ & 0.10 & 2.29 & 0.01 & 150.4140 & 6.975050 \\
\hline $5 \cdot 35$ & 0.10 & 2.30 & 0.01 & 151.8930 & 6.943370 \\
\hline
\end{tabular}


Table A.4 - Continued

\begin{tabular}{cccccc}
$E_{\text {beam }}^{\text {lab }}[\mathrm{GeV}]$ & $\pm d E_{\text {beam }}^{\text {lab }}$ & $M(p \bar{p})\left[\frac{\mathrm{GeV}}{c^{2}}\right]$ & $\pm d M(p \bar{p})$ & $\frac{d \sigma}{d M(p \bar{p})}[n b]$ & $\sigma^{\text {stat }}$ \\
\hline 5.35 & 0.10 & 2.31 & 0.01 & 138.3800 & 6.464250 \\
5.35 & 0.10 & 2.32 & 0.01 & 136.4260 & 6.431380 \\
5.35 & 0.10 & 2.33 & 0.01 & 127.9840 & 6.459670 \\
5.35 & 0.10 & 2.34 & 0.01 & 103.0830 & 5.617580 \\
5.35 & 0.10 & 2.35 & 0.01 & 97.5816 & 5.731780 \\
5.35 & 0.10 & 2.36 & 0.01 & 93.3655 & 5.855670 \\
5.35 & 0.10 & 2.37 & 0.01 & 71.1590 & 5.328920 \\
5.35 & 0.10 & 2.38 & 0.01 & 65.0913 & 5.297300 \\
5.35 & 0.10 & 2.39 & 0.01 & 39.0833 & 4.122720 \\
5.35 & 0.10 & 2.40 & 0.01 & 28.1831 & 4.353010 \\
5.35 & 0.10 & 2.41 & 0.01 & 17.3157 & 3.566140 \\
5.35 & 0.10 & 2.42 & 0.01 & 2.9069 & 2.920160 \\
\hline
\end{tabular}


VITA

WILLIAM B. PHELPS

2007-2011

2011-2016

2011-2017
B.S., Applied Physics

Christopher Newport University

Newport News, Virginia

M.S., Physics

Florida International University

Miami, Florida

Ph.D., Physics

Florida International University

Miami, Florida

PUBLICATIONS AND PRESENTATIONS

- "Antibaryon Photoproduction using CLAS at Jefferson lab" Talk. BARYON, Tallahassee FL, May 2016.

- W. Phelps and L. Guo on behalf of the CLAS Collaboration, Antibaryon Photoproduction using CLAS at Jefferson Lab. HADRON Conference Proceedings, 2015.

- "Antibaryon Photoproduction using CLAS at Jefferson lab" Talk. HADRON, Newport News VA, September 2015.

- "Antibaryon Photoproduction" Poster. Gordon Conference on Photonuclear Reactions, Holderness, NH, August 2014.

- "Antibaryon Photoproduction using CLAS at Jefferson lab" Talk. APS DNP Meeting, Newport News VA, October 2013. 